\title{
Total Synthesis of the Originally Proposed and Revised Structures of Palmerolide A and
}

\section{Isomers Thereof}

K.C. Nicolaou, Ya-Ping Sun, Ramakrishna Guduru, Biswadip Banerji, and David Y.-K Chen

Contribution from Chemical Synthesis Laboratory@Biopolis, Institute of Chemical and Engineering Sciences (ICES), Agency for Science, Technology and Research (A*STAR), 11 Biopolis Way, The Helios Block, \#03-08, Singapore 138667 (Singapore)

Email:kcn@scripps.edu; david_chen@ices.a-star.edu.sg

\section{Supporting Information Available}

I) Experimental section

II) Abbreviations

III) $\quad{ }^{1} \mathrm{H}$ and ${ }^{13} \mathrm{C}$ NMR spectra of compounds

\section{I) Experimental Section}

\section{Experimental Data for Compounds}

General Procedures. All reactions were carried out under a nitrogen or argon atmosphere with dry solvents under anhydrous conditions, unless otherwise noted. Dry tetrahydrofuran (THF), methylene chloride $\left(\mathrm{CH}_{2} \mathrm{Cl}_{2}\right)$, diethyl ether $\left(\mathrm{Et}_{2} \mathrm{O}\right)$, toluene and triethylamine $\left(\mathrm{Et}_{3} \mathrm{~N}\right)$ were obtained by passing commercially available pre-dried, oxygen-free formulations through activated alumina columns. $N, N^{\prime}$-dimethylformamide (DMF) was purchased in anhydrous form and used without further purification. Water $\left(\mathrm{H}_{2} \mathrm{O}\right)$, methanol $(\mathrm{MeOH})$, ethanol $(\mathrm{EtOH})$, ethyl acetate (EtOAc), chloroform $\left(\mathrm{CHCl}_{3}\right)$, 1,2-dichloroethane, dioxane and benzene were purchased at the highest commercial quality and used without further purification, unless otherwise stated. Reagents were purchased at the highest commercial quality and used without further purification, unless otherwise stated. Yields refer to chromatographically and spectroscopically ( ${ }^{1} \mathrm{H}$ NMR) 
homogeneous materials, unless otherwise stated. Reactions were monitored by thin-layer chromatography (TLC) carried out on $0.25 \mathrm{~mm}$ E. Merck silica gel plates (60F-254) using UV light as visualizing agent and an ethanolic solution of ammonium molybdate and anisaldehyde and heat as developing agents. E. Merck silica gel (60, particle size $0.040-0.063 \mathrm{~mm})$ was used for flash column chromatography. Preparative thin-layer chromatography separations were carried out on 0.25 or $2.0 \mathrm{~mm}$ E. Merck silica gel plates (60F-254). NMR spectra were recorded on Bruker AV-600 instrument and calibrated using residual undeuterated solvent as an internal reference. The following abbreviations were used to explain the multiplicities: $s=$ singlet, $d=$ doublet, $\mathrm{t}=$ triplet, $\mathrm{q}=$ quartet, $\mathrm{m}=$ multiplet, pent $=$ pentet, $\mathrm{hex}=$ hexet, $\mathrm{br}=$ broad. IR spectra were recorded on a Perkin-Elmer Spectrum One FTIR spectrometer with diamond ATR accessory. Optical rotation $\left([\alpha]_{\mathrm{D}}\right)$ were recorded on a Perkin-Elmer Model 341 polarimeter at 25 ${ }^{\circ} \mathrm{C}$ using thermostable optical glass cell (100 mm path length). LC/MS data were recorded on an Agilent 1100 series LC system coupled to a multimode ESI/APCI Agilent MSD. High-resolution mass spectra (HRMS) were recorded on an Agilent ESI TOF (time of flight) mass spectrometer at $3500 \mathrm{~V}$ emitter voltage.

Alcohol 8: To a solution of (R)-4-benzyl-3-propionyloxazolidinone 6 (389 $\mathrm{mg}, 1.67 \mathrm{mmol})$ in $\mathrm{CH}_{2} \mathrm{Cl}_{2}(5 \mathrm{~mL})$ were added $n-\mathrm{Bu}_{2} \mathrm{BOTf}\left(1.0 \mathrm{M}\right.$ in $\left.\mathrm{CH}_{2} \mathrm{Cl}_{2}, 2 \mathrm{~mL}, 2.00 \mathrm{mmol}\right)$ and $\mathrm{Et}_{3} \mathrm{~N}(0.23 \mathrm{~mL}$,<smiles>C/C(=C\I)C[C@@H](O)C(C)C(=O)N1C(=O)OC[C@H]1Br</smiles>
$1.67 \mathrm{mmol})$ at $0{ }^{\circ} \mathrm{C}$. The reaction mixture was stirred for $15 \mathrm{~min}$ before a solution of aldehyde $7(883 \mathrm{mg}, 4.18 \mathrm{mmol})$ in $\mathrm{CH}_{2} \mathrm{Cl}_{2}(5 \mathrm{~mL})$ was added at $-78{ }^{\circ} \mathrm{C}$. The reaction mixture was warmed to room temperature and stirred for $12 \mathrm{~h}$ before it was quenched with phosphate buffer $(5 \mathrm{~mL})$. The resulting mixture was diluted with a mixture of $\mathrm{MeOH} / \mathrm{H}_{2} \mathrm{O}(2: 1,2 \mathrm{~mL})$ and stirred for $1 \mathrm{~h}$ before it was concentrated 
in vacuo. The crude residue was extracted with EtOAc $(3 \times 20 \mathrm{~mL})$, and the combined organic layers were washed with $\mathrm{NaHCO}_{3}\left(10 \mathrm{~mL}\right.$, sat. aq.), brine $(10 \mathrm{~mL})$, dried $\left(\mathrm{Na}_{2} \mathrm{SO}_{4}\right)$ and concentrated in vacuo. Flash column chromatography (silica gel, hexanes:EtOAc 4:1) afforded alcohol 8 (340 mg, 46\%) as a pale yellow oil. 8: $R_{\mathrm{f}}=0.35$ (silica gel, hexanes:EtOAc 4:1); $[\alpha]_{\mathrm{D}}{ }^{25}$ $=+21.3\left(\mathrm{CHCl}_{3}, c=1.00\right)$; IR (film) $v_{\max } 3507,2920,1777,1697,1386,1212,1108,983 \mathrm{~cm}^{-1}$; ${ }^{1} \mathrm{H}$ NMR (600 MHz, $\left.\mathrm{CDCl}_{3}\right): \delta=7.35(\mathrm{~m}, 2 \mathrm{H}), 7.28(\mathrm{~m}, 1 \mathrm{H}), 7.22(\mathrm{~m}, 2 \mathrm{H}), 6.07(\mathrm{~s}, 1 \mathrm{H}), 4.72$ (m, $1 \mathrm{H}), 4.28(\mathrm{dd}, J=7.8,7.2 \mathrm{~Hz}, 1 \mathrm{H}), 4.21(\mathrm{dd}, J=7.8,0.6 \mathrm{~Hz}, 1 \mathrm{H}), 4.15(\mathrm{~m}, 1 \mathrm{H}), 3.79$ (dd, $J=7.2,3.6 \mathrm{~Hz}, 1 \mathrm{H}), 3.26$ (dd, $J=13.2,3.6 \mathrm{~Hz}, 1 \mathrm{H}), 2.80$ (dd, $J=13.8,9.6 \mathrm{~Hz}, 1 \mathrm{H}), 2.60$ (bs, $1 \mathrm{H}), 2.49$ (ddd, $J=13.8,9.0,1.2 \mathrm{~Hz}, 1 \mathrm{H}), 2.36(\mathrm{ddd}, J=9.0,4.2,1.2 \mathrm{~Hz}, 1 \mathrm{H}), 1.91(\mathrm{~d}, J=1.2$ $\mathrm{Hz}, 3 \mathrm{H}), 1.30 \mathrm{ppm}(\mathrm{d}, J=6.6 \mathrm{~Hz}, 3 \mathrm{H}) ;{ }^{13} \mathrm{C} \mathrm{NMR}\left(150 \mathrm{MHz}, \mathrm{CDCl}_{3}\right): \delta=176.3,153.2,144.7$, 135.1, 129.4, 129.0, 127.5, 77.5, 69.4, 66.3, 55.3, 44.0, 42.1, 38.1, 24.1, 10.5 ppm; HRMS (ESI): calcd for $\mathrm{C}_{18} \mathrm{H}_{22} \mathrm{INO}_{4} \mathrm{Na}^{+}\left[\mathrm{M}+\mathrm{Na}^{+}\right]$466.0486, found 466.0500.

TBS ether 9: To a solution of alcohol $8(100 \mathrm{mg}, 0.23 \mathrm{mmol})$ in $\mathrm{CH}_{2} \mathrm{Cl}_{2}(3 \mathrm{~mL})$ were added $i$ $\operatorname{Pr}_{2} \mathrm{NEt}(61 \mu \mathrm{L}, 0.35 \mathrm{mmol})$ and $\operatorname{TBSOTf}(63 \mu \mathrm{L}, 0.34 \mathrm{mmol})$ at $0{ }^{\circ} \mathrm{C}$. The reaction mixture was mixture was extracted with $\mathrm{CH}_{2} \mathrm{Cl}_{2}(2 \times 10 \mathrm{~mL})$, and the combined organic vacuo. Flash column chromatography (silica gel, hexanes:EtOAc 9:1) afforded TBS ether 9 (105 mg, 84\%) as a colorless oil. 9: $R_{\mathrm{f}}=0.38$ (silica gel, hexanes:EtOAc 9:1); $[\alpha]_{\mathrm{D}}^{25}=+0.6\left(\mathrm{CHCl}_{3}, c=1.88\right)$; IR (film) $v_{\max } 2929,2857,1780,1698,1382,1207,1109$, $836 \mathrm{~cm}^{-1} ;{ }^{1} \mathrm{H}$ NMR $\left(600 \mathrm{MHz}, \mathrm{CDCl}_{3}\right): \delta=7.36(\mathrm{~m}, 2 \mathrm{H}), 7.23(\mathrm{~m}, 2 \mathrm{H}), 5.98(\mathrm{~s}, 1 \mathrm{H}), 4.64(\mathrm{ddd}$, $J=14.1,7.2,3.6 \mathrm{~Hz}, 1 \mathrm{H}), 4.23(\mathrm{dd}, J=11.4,6.6 \mathrm{~Hz}, 1 \mathrm{H}), 4.16(\mathrm{~m}, 2 \mathrm{H}), 3.86(\mathrm{dt}, J=13.8,7.2$ 
$\mathrm{Hz}, 1 \mathrm{H}), 3.47$ (dd, $J=6.6,3.6 \mathrm{~Hz}, 1 \mathrm{H}), 2.67$ (dd, $J=13.2,10.8 \mathrm{~Hz}, 1 \mathrm{H}), 2.58(\mathrm{dd}, J=13.8,6.6$ Hz, $1 \mathrm{H}), 2.48(\mathrm{dd}, J=13.2,4.2 \mathrm{~Hz}, 1 \mathrm{H}), 1.91(\mathrm{~s}, 3 \mathrm{H}), 1.58(\mathrm{~s}, 3 \mathrm{H}), 1.22(\mathrm{~d}, J=7.2 \mathrm{~Hz}, 1 \mathrm{H})$, $0.92(\mathrm{~s}, 9 \mathrm{H}), 0.08(\mathrm{~s}, 3 \mathrm{H}), 0.07 \mathrm{ppm}(\mathrm{s}, 3 \mathrm{H}) ;{ }^{13} \mathrm{C} \mathrm{NMR}\left(150 \mathrm{MHz}, \mathrm{CDCl}_{3}\right): \delta=175.3,153.1$, 144.8, 135.6, 129.3, 129.0, 127.3, 78.6, 71.2, 66.1, 55.7, 46.5, 43.4, 38.6, 25.9, 24.2, 18.1, 13.6, $-3.9,-4.4$ ppm; HRMS (ESI): calcd for $\mathrm{C}_{24} \mathrm{H}_{36} \mathrm{INO}_{4} \mathrm{SiNa}^{+}\left[\mathrm{M}+\mathrm{Na}^{+}\right]$580.1351, found 580.1361.

Alcohol 10: To a solution of imide 9 (100 $\mathrm{mg}, 0.18 \mathrm{mmol})$ in THF $(3 \mathrm{~mL})$ was added $\mathrm{NaBH}_{4}(34$ $\mathrm{mg}, 0.90 \mathrm{mmol}$ ) at $0{ }^{\circ} \mathrm{C}$. The reaction mixture was warmed to room temperature and stirred for 3

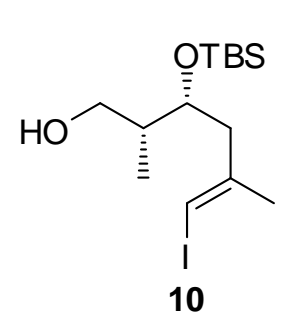
h before it was quenched with $\mathrm{HCl}(1.0 \mathrm{~N}$ aq., $2.0 \mathrm{~mL})$. The resulting mixture was extracted with EtOAc $(3 \times 10 \mathrm{~mL})$, and the combined organic layers were washed with brine $(5 \mathrm{~mL})$, dried $\left(\mathrm{Na}_{2} \mathrm{SO}_{4}\right)$ and concentrated in vacuo. Flash column chromatography (silica gel, hexanes:EtOAc 9:1) afforded alcohol 10 $(49 \mathrm{mg}, 66 \%)$ as a colorless oil. 10: $R_{\mathrm{f}}=0.65$ (silica gel, hexanes:EtOAc 9:1); $[\alpha]_{\mathrm{D}}{ }^{25}=-7.8$ $\left(\mathrm{CHCl}_{3}, c=0.98\right)$; IR (film) $v_{\max } 3360,2929,1472,1377,1076,835 \mathrm{~cm}^{-1} ;{ }^{1} \mathrm{H}$ NMR $(600 \mathrm{MHz}$, $\left.\mathrm{CDCl}_{3}\right): \delta=5.98(\mathrm{~s}, 1 \mathrm{H}), 3.95(\mathrm{ddd}, J=8.4,6.0,3.0 \mathrm{~Hz}, 1 \mathrm{H}), 3.66(\mathrm{dd}, J=10.8,9.0 \mathrm{~Hz}, 1 \mathrm{H})$, $3.54(\mathrm{dd}, J=10.8,5.4 \mathrm{~Hz}, 1 \mathrm{H}), 2.41(\mathrm{~m}, 2 \mathrm{H}), 1.93(\mathrm{~m}, 1 \mathrm{H}), 1.85(\mathrm{~s}, 3 \mathrm{H}), 0.90$ (s, 9 H), 0.84 (d, $J=6.6 \mathrm{~Hz}, 3 \mathrm{H}), 0.09(\mathrm{~s}, 3 \mathrm{H}), 0.04 \mathrm{ppm}(\mathrm{s}, 3 \mathrm{H}) ;{ }^{13} \mathrm{C} \mathrm{NMR}\left(150 \mathrm{MHz}, \mathrm{CDCl}_{3}\right): \delta=144.4,77.9$, 72.4, 65.5, 43.1, 39.6, 25.8, 24.1, 18.0, 11.5, -4.4, -4.7 ppm; HRMS (ESI): calcd for $\mathrm{C}_{14} \mathrm{H}_{30} \mathrm{IO}_{2} \mathrm{Si}^{+}\left[\mathrm{M}+\mathrm{H}^{+}\right]$385.1054, found 385.1054.

Aldehyde 11: To a solution of alcohol $10(49 \mathrm{mg}, 0.13 \mathrm{mmol})$ in $\mathrm{CH}_{2} \mathrm{Cl}_{2}(2 \mathrm{~mL})$ were added $\mathrm{NaHCO}_{3}(54 \mathrm{mg}, 0.63 \mathrm{mmol})$ and DMP $(66 \mathrm{mg}, 0.16 \mathrm{mmol})$ at room temperature. The reaction mixture was stirred for 30 min before it was quenched with $\mathrm{NaHCO}_{3}(2.0 \mathrm{~mL}$, sat. aq.) and 


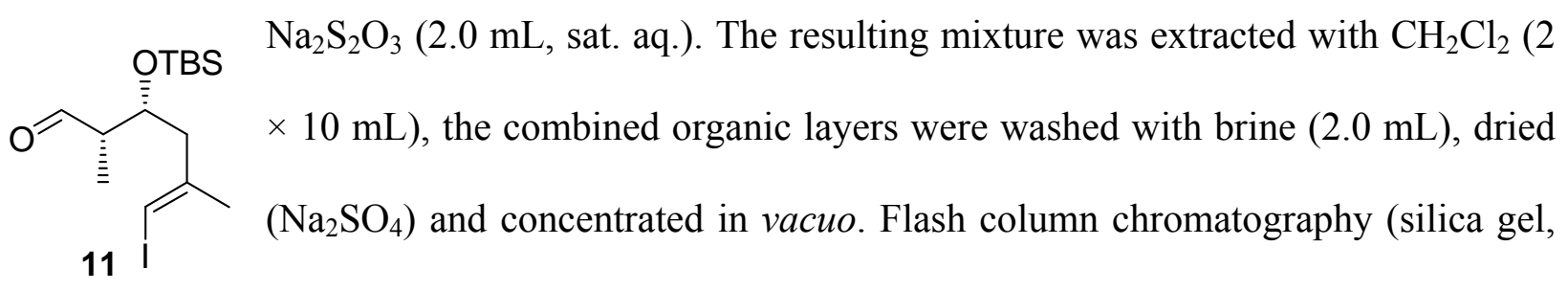
hexanes:EtOAc 9:1) afforded aldehyde $\mathbf{1 1}(42 \mathrm{mg}, 85 \%)$ as a colorless oil. 11:

$R_{\mathrm{f}}=0.41$ (silica gel, hexanes:EtOAc 19:1); $[\alpha]_{\mathrm{D}}^{25}=-33.5\left(\mathrm{CHCl}_{3}, c=2.00\right)$; IR (film) $v_{\max } 2929$, 2712, 1726, 1472, 1252, 1034, 834, $775 \mathrm{~cm}^{-1} ;{ }^{1} \mathrm{H}$ NMR (600 MHz, $\left.\mathrm{CDCl}_{3}\right): \delta=9.77(\mathrm{~s}, 1 \mathrm{H})$, $6.00(\mathrm{~s}, 1 \mathrm{H}), 4,30(\mathrm{ddd}, J=9.6,6.6,3.0 \mathrm{~Hz}, 1 \mathrm{H}), 2.49-2.33(\mathrm{~m}, 3 \mathrm{H}), 1.88(\mathrm{~s}, 3 \mathrm{H}), 1.11(\mathrm{~d}, J=$ $6.6 \mathrm{~Hz}, 3 \mathrm{H}), 0.87(\mathrm{~s}, 9 \mathrm{H}), 0.06(\mathrm{~s}, 3 \mathrm{H}), 0.05 \mathrm{ppm}(\mathrm{s}, 3 \mathrm{H}) ;{ }^{13} \mathrm{C} \mathrm{NMR}\left(150 \mathrm{MHz}, \mathrm{CDCl}_{3}\right): \delta=$ 204.6, 143.9, 78.6, 69.6, 51.0, 44.9, 25.7, 24.2, 18.0, 7.6, -4.3, -4.7 ppm; HRMS (ESI): calcd for $\mathrm{C}_{14} \mathrm{H}_{27} \mathrm{IO}_{2} \mathrm{SiNa}^{+}\left[\mathrm{M}+\mathrm{Na}^{+}\right]$405.0717, found 405.0708.

Enoate 12: To a solution aldehyde $11(42 \mathrm{mg}, 0.10 \mathrm{mmol})$ in $\mathrm{CH}_{2} \mathrm{Cl}_{2}(2 \mathrm{~mL})$ was added $\mathrm{PPh}_{3}=\mathrm{C}(\mathrm{Me}) \mathrm{CO}_{2} \mathrm{Et}(108 \mathrm{mg}, 0.30 \mathrm{mmol})$ at room temperature. The reaction mixture was stirred for $12 \mathrm{~h}$ before it was concentrated in vacuo. Flash column
$\mathrm{mg}, 66 \%$ ) as a colorless oil. 12: $R_{\mathrm{f}}=0.46$ (silica gel, hexanes:EtOAc 19:1); $[\alpha]_{\mathrm{D}}^{25}=-35.8\left(\mathrm{CHCl}_{3}, c=1.0\right)$; IR (film) $\nu_{\max } 2930,1712,1463,1367,1254,1104,836$, $775 \mathrm{~cm}^{-1} ;{ }^{1} \mathrm{H}$ NMR $\left(600 \mathrm{MHz}, \mathrm{CDCl}_{3}\right): \delta=6.67(\mathrm{~d}, J=10.2 \mathrm{~Hz}, 1 \mathrm{H}), 5.95(\mathrm{~s}, 1 \mathrm{H}), 4.25-4.15$ (m, $4 \mathrm{H}), 3.73$ (ddd, $J=10.8,6.0,4.8 \mathrm{~Hz}, 1 \mathrm{H}), 2.54$ (m, $1 \mathrm{H}), 2.42$ (dd, $J=13.8,6.0 \mathrm{~Hz}, 1 \mathrm{H})$, $2.36(\mathrm{dd}, J=13.8,6.6 \mathrm{~Hz}, 1 \mathrm{H}), 1.85(\mathrm{~s}, 3 \mathrm{H}), 1.83(\mathrm{~s}, 3 \mathrm{H}), 1.30(\mathrm{t}, J=7.2 \mathrm{~Hz}, 3 \mathrm{H}), 0.99$ (d, $J=$ $6.6 \mathrm{~Hz}, 3 \mathrm{H}), 0.90(\mathrm{~s}, 9 \mathrm{H}), 0.05(\mathrm{~s}, 3 \mathrm{H}), 0.02 \mathrm{ppm}(\mathrm{s}, 3 \mathrm{H}) ;{ }^{13} \mathrm{C} \mathrm{NMR}\left(150 \mathrm{MHz}, \mathrm{CDCl}_{3}\right): \delta=$ $162.2,144.7,144.4,127.0,78.0,73.1,60.5,45.4,38.2,25.8,24.4,18.1,14.3,14.1,12.6,-4.1$, -4.6 ppm; HRMS (ESI): calcd for $\mathrm{C}_{19} \mathrm{H}_{35} \mathrm{IO}_{3} \mathrm{SiNa}^{+}\left[\mathrm{M}+\mathrm{Na}^{+}\right]$489.1292, found 489.1283. 
Alcohol 13: To a solution of enoate $12(28 \mathrm{mg}, 0.06 \mathrm{mmol})$ in $\mathrm{CH}_{2} \mathrm{Cl}_{2}(2 \mathrm{~mL})$ was added DIBAL-H (1.0 M in toluene, $0.15 \mathrm{~mL}, 0.15 \mathrm{mmol})$ at $-78{ }^{\circ} \mathrm{C}$. The reaction mixture was stirred

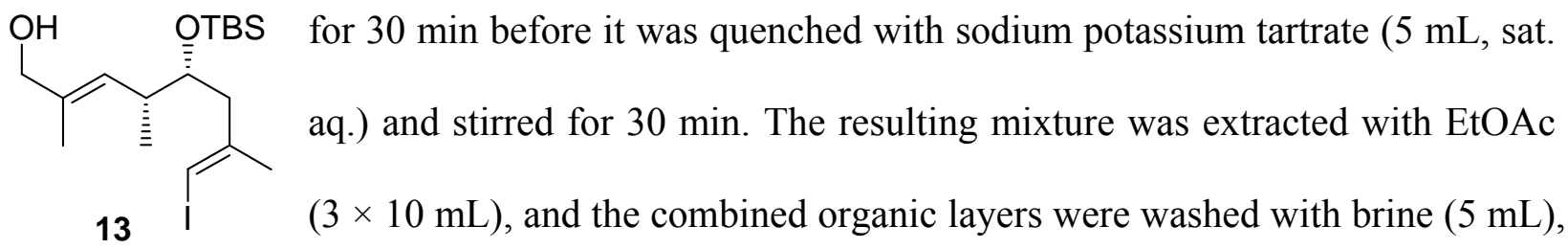
dried $\left(\mathrm{Na}_{2} \mathrm{SO}_{4}\right)$ and concentrated in vacuo. Flash column chromatography (silica gel, hexanes:EtOAc 9:1) afforded alcohol $13(21 \mathrm{mg}, 80 \%)$ as a colorless oil. 13: $R_{\mathrm{f}}=0.35$ (silica gel, hexanes:EtOAc 9:1); $[\alpha]_{\mathrm{D}}^{25}=-11.6\left(\mathrm{CHCl}_{3}, c=0.85\right)$; IR (film) $v_{\max } 3327,2929,1472,1462$, 1377, 1255, 1079, 1005, $836 \mathrm{~cm}^{-1} ;{ }^{1} \mathrm{H}$ NMR (600 MHz, $\left.\mathrm{CDCl}_{3}\right): \delta=5.93(\mathrm{~s}, 1 \mathrm{H}), 5.31(\mathrm{~d}, J=$ $10.2 \mathrm{~Hz}, 1 \mathrm{H}), 4.01(\mathrm{~s}, 2 \mathrm{H}), 3.66(\mathrm{ddd}, J=10.8,5.4,5.4 \mathrm{~Hz}, 1 \mathrm{H}), 2.47(\mathrm{~m}, 1 \mathrm{H}), 2.46(\mathrm{dd}, J=$ 13.2, $5.4 \mathrm{~Hz}, 1 \mathrm{H}), 2.34(\mathrm{dd}, J=13.2,6.6 \mathrm{~Hz}, 1 \mathrm{H}), 1.85(\mathrm{~d}, J=0.6 \mathrm{~Hz}, 3 \mathrm{H}), 1.66(\mathrm{~d}, J=1.2 \mathrm{~Hz}$, $3 \mathrm{H}), 0.95(\mathrm{~d}, J=6.6 \mathrm{~Hz}, 3 \mathrm{H}), 0.90(\mathrm{~s}, 9 \mathrm{H}), 0.04(\mathrm{~s}, 3 \mathrm{H}), 0.02 \mathrm{ppm}(\mathrm{s}, 3 \mathrm{H}) ;{ }^{13} \mathrm{C}$ NMR $(150$ $\left.\mathrm{MHz}, \mathrm{CDCl}_{3}\right): \delta=144.9,134.3,129.3,77.6,74.0,68.9,45.2,37.3,25.9,24.5,18.1,15.6,14.0$ -4.1, -4.4 ppm; HRMS (ESI): calcd for $\mathrm{C}_{17} \mathrm{H}_{33} \mathrm{IO}_{2} \mathrm{SiNa}^{+}\left[\mathrm{M}+\mathrm{Na}^{+}\right]$447.1187, found 447.1173

Diol 14: To a solution of TBS ether $13(21 \mathrm{mg}, 49.0 \mu \mathrm{mol})$ in THF $(2 \mathrm{~mL})$ was added TBAF (1.0 $\mathrm{M}$ in THF, $74 \mu \mathrm{L}, 74 \mu \mathrm{mol})$ at room temperature. The reaction mixture was heated at reflux for 2 $\mathrm{HO} \quad \mathrm{OH} \quad \mathrm{h}$ before it was cooled to room temperature and concentrated in vacuo. The crude residue was diluted with water $(2 \mathrm{~mL})$, extracted with EtOAc $(3 \times 10$ $\mathrm{mL})$, and the combined organic layers were washed with brine $(5 \mathrm{~mL})$, dried 14

$\left(\mathrm{Na}_{2} \mathrm{SO}_{4}\right)$ and concentrated in vacuo. Flash column chromatorgraphy (silica gel, hexanes:EtOAc 8:2) afforded diol $14\left(13.1 \mathrm{mg}, 85 \%\right.$ ) as a viscous oil. 14: $R_{\mathrm{f}}=0.23$ (silica 
gel, hexanes:EtOAc 4:1); $[\alpha]_{\mathrm{D}}^{25}=+16.2\left(\mathrm{CHCl}_{3}, c=2.0\right)$; IR (film) $v_{\max } 3327,2913,1451,1377$, $1272,1007 \mathrm{~cm}^{-1} ;{ }^{1} \mathrm{H} \mathrm{NMR}\left(600 \mathrm{MHz}, \mathrm{CDCl}_{3}\right): \delta=6.01(\mathrm{~s}, 1 \mathrm{H}), 5.29(\mathrm{dq}, J=10.2,1.2 \mathrm{~Hz}, 1 \mathrm{H})$, 4.03 (s, $2 \mathrm{H}$ ), 3.57 (ddd, $J=10.2,6.6,3.0 \mathrm{~Hz}, 1 \mathrm{H}), 2.50$ (ddq, $J=10.2,10.2,6.8 \mathrm{~Hz}, 1 \mathrm{H}), 2.43$ (dd, $J=13.8,2.4 \mathrm{~Hz}, 1 \mathrm{H}), 2.25(\mathrm{dd}, J=13.8,9.6 \mathrm{~Hz}, 1 \mathrm{H}), 1.88(\mathrm{~s}, 3 \mathrm{H}), 1.69(\mathrm{~d}, J=1.2 \mathrm{~Hz}, 3$ H), $1.09 \mathrm{ppm}(\mathrm{d}, J=6.6 \mathrm{~Hz}, 3 \mathrm{H}) ;{ }^{13} \mathrm{C} \mathrm{NMR}\left(150 \mathrm{MHz}, \mathrm{CDCl}_{3}\right): \delta=145.3$, 135.6, 127.6, 77.0, 72.9, 68.5, 44.7, 38.0, 24.0, 16.2, 14.2 ppm; HRMS (ESI): calcd for $\mathrm{C}_{11} \mathrm{H}_{19} \mathrm{IO}_{2} \mathrm{Na}^{+}\left[\mathrm{M}+\mathrm{Na}^{+}\right]$ 333.0322, found 333.0318.

TBS ether 2: To a solution of diol $14(13.0 \mathrm{mg}, 41.9 \mu \mathrm{mol})$ in $\mathrm{CH}_{2} \mathrm{Cl}_{2}(2.0 \mathrm{~mL})$ were added $\mathrm{Et}_{3} \mathrm{~N}(9 \mu \mathrm{L}, 62.9 \mu \mathrm{mol}), \mathrm{TBSCl}(7.5 \mathrm{mg}, 50.8 \mu \mathrm{mol})$ and 4-DMAP $(1.0 \mathrm{mg}, 8.3 \mu \mathrm{mol})$ at $0{ }^{\circ} \mathrm{C}$. The reaction mixture was warmed to room temperature and stirred for $2 \mathrm{~h}$

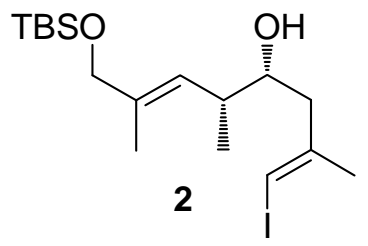
before it was quenched with water $(1 \mathrm{~mL})$. The resulting mixture was extracted with EtOAc $(3 \times 5 \mathrm{~mL})$, and the combined organic layers were dried $\left(\mathrm{Na}_{2} \mathrm{SO}_{4}\right)$ and concentrated in vacuo. Flash column chromatography (silica gel, hexanes:EtOAc 10:1) afforded TBS ether $2(16.4 \mathrm{mg}, 92 \%)$ as a colorless oil. $2: R_{\mathrm{f}}=$ 0.33 (silica gel, hexanes:EtOAc 8:1); $[\alpha]_{\mathrm{D}}^{25}=+21.6\left(\mathrm{CH}_{2} \mathrm{Cl}_{2}, c=0.80\right.$ ); IR (film) $v_{\max } 3428$, 2955, 2929, 1462, 1254, $1074 \mathrm{~cm}^{-1} ;{ }^{1} \mathrm{H}$ NMR (600 MHz, $\left.\mathrm{CDCl}_{3}\right): \delta=5.99(\mathrm{~s}, 1 \mathrm{H}), 5.25(\mathrm{~d}, J=$ $10.2 \mathrm{~Hz}, 1 \mathrm{H}), 4.02(\mathrm{~s}, 2 \mathrm{H}), 3.51(\mathrm{ddd}, J=7.8,4.2,1.2 \mathrm{~Hz}, 1 \mathrm{H}), 2.48(\mathrm{dqd}, J=10.2,6.6,4.8 \mathrm{~Hz}$, $1 \mathrm{H}), 2.44(\mathrm{dd}, J=13.2,1.2 \mathrm{~Hz}, 1 \mathrm{H}), 2.21(\mathrm{dd}, J=13.8,9.6 \mathrm{~Hz}, 1 \mathrm{H}), 1.86(\mathrm{~s}, 3 \mathrm{H}), 1.62(\mathrm{~s}, 3 \mathrm{H})$, $1.03(\mathrm{~d}, J=6.6 \mathrm{~Hz}, 3 \mathrm{H}), 0.92(\mathrm{~s}, 9 \mathrm{H}), 0.07 \mathrm{ppm}(\mathrm{s}, 6 \mathrm{H}) ;{ }^{13} \mathrm{C}$ NMR $\left(150 \mathrm{MHz}, \mathrm{CDCl}_{3}\right): \delta=$ 145.5, 135.6, 126.0, 76.8, 73.1, 68.2, 45.1, 38.2, 25.9, 24.0, 18.4, 16.6, 13.9, -5.2 ppm; HRMS (ESI): calcd for $\mathrm{C}_{17} \mathrm{H}_{33} \mathrm{IO}_{2} \mathrm{SiNa}^{+}\left[\mathrm{M}+\mathrm{Na}^{+}\right]$447.1187, found 447.1194. 
Imide 15: To a solution of E-2-methyl-2-pentenoic acid (6.8 g, $59.6 \mathrm{mmol}), \mathrm{Et}_{3} \mathrm{~N}$ (21 mL, 149.0 mmol $)$ in $\mathrm{CH}_{2} \mathrm{Cl}_{2}(100 \mathrm{~mL})$ was added trimethylacetyl chloride $(7.2 \mathrm{~g}, 59.6 \mathrm{mmol})$ at $-78{ }^{\circ} \mathrm{C}$.<smiles>CC/C=C(\C)C(=O)N1C(=O)OC[C@H]1Br</smiles>
The reaction mixture was warmed to room temperature and stirred for $1 \mathrm{~h}$ before a solution of $(S)-(+)-4-$ benzyl-2-oxazolidinone $(10.5 \mathrm{~g}, 59.6 \mathrm{mmol})$ in $\mathrm{CH}_{2} \mathrm{Cl}_{2}$ $(30 \mathrm{~mL})$ and $\mathrm{LiCl}(3.7 \mathrm{~g}, 88.1 \mathrm{mmol})$ were added at $-78{ }^{\circ} \mathrm{C}$. The reaction mixture was warmed to room temperature and stirred for $12 \mathrm{~h}$ before it was diluted with $\mathrm{H}_{2} \mathrm{O}(50$ $\mathrm{mL})$. The resulting mixture was extracted with $\mathrm{CH}_{2} \mathrm{Cl}_{2}(2 \times 50 \mathrm{~mL})$, and the combined organic layers were washed with brine $(50 \mathrm{~mL})$, dried $\left(\mathrm{Na}_{2} \mathrm{SO}_{4}\right)$ and concentrated in vacuo. Flash column chromatography (silica gel, hexanes:EtOAc 4:1) afforded imide $\mathbf{1 5}(14.6 \mathrm{~g}, 91 \%)$ as a viscous oil. 15: $R_{\mathrm{f}}=0.35$ (silica gel, hexanes:EtOAc 4:1); $[\alpha]_{\mathrm{D}}^{25}=-48.2\left(\mathrm{CHCl}_{3}, c=0.86\right)$; IR (film) $v_{\max }$ $2972,1785,1682,1455,1353,1313,1212,1046 \mathrm{~cm}^{-1} ;{ }^{1} \mathrm{H}$ NMR $\left(600 \mathrm{MHz}, \mathrm{CDCl}_{3}\right): \delta=$ 7.35-7.29 (m, 3 H), 7.22-7.21 (m, 2 H), 6.10-6.08 (m, 1 H), 4.75-4.72 (m, 1 H), 4.27-4.24 (m, $1 \mathrm{H}), 4.16$ (dd, $J=7.2,5.4 \mathrm{~Hz}, 1 \mathrm{H}), 3.63(\mathrm{dd}, J=8.4,3.0 \mathrm{~Hz}, 1 \mathrm{H}), 2.84(\mathrm{dd}, J=9.0,4.2 \mathrm{~Hz}, 1$ H), 2.23 (q, $J=7.2 \mathrm{~Hz}, 2 \mathrm{H}), 1.91(\mathrm{~s}, 3 \mathrm{H}), 1.07 \mathrm{ppm}(\mathrm{t}, J=7.2 \mathrm{~Hz}, 3 \mathrm{H}) ;{ }^{13} \mathrm{C}$ NMR (150 MHz, $\left.\mathrm{CDCl}_{3}\right): \delta=172.0,153.2,141.6,135.2,130.0,129.5,128.9,127.3,66.4,55.5,37.6,21.7,31.3$, 12.8 ppm; HRMS (ESI): calcd for $\mathrm{C}_{16} \mathrm{H}_{19} \mathrm{O}_{3} \mathrm{NNa}^{+}\left[\mathrm{M}+\mathrm{Na}^{+}\right]$273.1365, found 273.1389.

Dienolate 16: To a solution of imide $15(11.5 \mathrm{~g}, 42.0 \mathrm{mmol})$ in THF $(100 \mathrm{~mL})$ was added NaHMDS (1.0 M in THF, $63.0 \mathrm{~mL}, 63.0 \mathrm{mmol})$ at $-78{ }^{\circ} \mathrm{C}$. The reaction mixture was stirred for

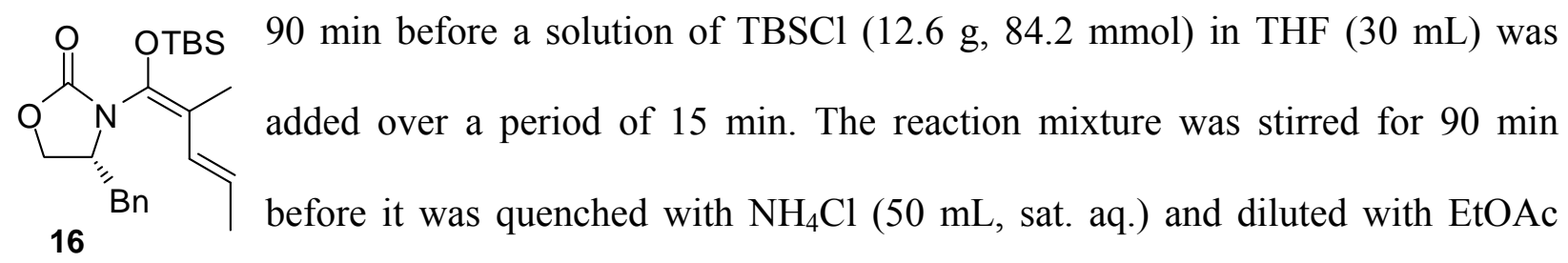
$(200 \mathrm{~mL})$. The resulting mixture was extracted with EtOAc $(3 \times 50 \mathrm{~mL})$, and the combined 
organic layers were washed with brine $(50 \mathrm{~mL})$, dried $\left(\mathrm{Na}_{2} \mathrm{SO}_{4}\right)$ and concentrated in vacuo. Flash column chromatography (silica gel, hexanes:EtOAc 9:1) afforded dienolate 16 (15.5 g, 95\%) as a viscous oil. 16: $R_{\mathrm{f}}=0.23$ (silica gel, hexanes:EtOAc 4:1); $[\alpha]_{\mathrm{D}}{ }^{25}=+78.3\left(\mathrm{CHCl}_{3}, c=1.4\right.$ ); IR (film) $v_{\max } 2930,1763,1630,1396,1376,1254,1163,1069,838 \mathrm{~cm}^{-1} ;{ }^{1} \mathrm{H}$ NMR (600 MHz, $\left.\mathrm{CDCl}_{3}\right): \delta=7.32(\mathrm{~m}, 2 \mathrm{H}), 7.26(\mathrm{~m}, 2 \mathrm{H}), 7.15(\mathrm{~d}, J=7.2 \mathrm{~Hz}, 1 \mathrm{H}), 6.19(\mathrm{~d}, J=15.6 \mathrm{~Hz}, 1 \mathrm{H})$, $5.70(\mathrm{~m}, 1 \mathrm{H}), 4.31-4.23(\mathrm{bm}, 2 \mathrm{H}), 4.15(\mathrm{bs}, 1 \mathrm{H}), 3.14(\mathrm{~d}, J=13.2 \mathrm{~Hz}, 1 \mathrm{H}), 2.63(\mathrm{bm}, 1 \mathrm{H})$, $1.83(\mathrm{~s}, 6 \mathrm{H}), 1.01$ (s, $9 \mathrm{H}), 0.23(\mathrm{~s}, 3 \mathrm{H}), 0.16 \mathrm{ppm}(\mathrm{s}, 3 \mathrm{H}) ;{ }^{13} \mathrm{C} \mathrm{NMR}\left(150 \mathrm{MHz}, \mathrm{CDCl}_{3}\right): \delta=$ $155.2,135.7,133.7,129.0 \times 2,128.8 \times 2,127.9,127.1,124.9,116.3,67.8,56.2,38.8,25.7 \times 3$, 18.8, 18.1, 12.3, $-4.3,-4.9$ ppm; HRMS (ESI): calcd for $\mathrm{C}_{22} \mathrm{H}_{33} \mathrm{NO}_{3} \mathrm{SiNa}^{+}\left[\mathrm{M}+\mathrm{Na}^{+}\right] 410.2122$, found 410.2133 .

Alcohol 17: To a solution of aldehyde $7(568 \mathrm{mg}, 2.60 \mathrm{mmol})$ in $\mathrm{CH}_{2} \mathrm{Cl}_{2}(6.5 \mathrm{~mL})$ were added $\mathrm{TiCl}_{4}(0.143 \mathrm{~mL}, 1.30 \mathrm{mmol})$ and a solution of dienolate $16(503 \mathrm{mg}, 1.30 \mathrm{mmol})$ in $\mathrm{CH}_{2} \mathrm{Cl}_{2}(6.5$<smiles>C/C=C(\C)C[C@@H](O)/C(C)=C/C(=O)N1C(=O)OC[C@H]1Br</smiles>
$\mathrm{mL})$ at $-78{ }^{\circ} \mathrm{C}$. The reaction mixture was stirred for $19 \mathrm{~h}$ before it was quenched with $\mathrm{NaHCO}_{3}(20 \mathrm{~mL}$, sat. aq.). The resulting mixture was extracted with EtOAc $(3 \times 30 \mathrm{~mL})$, and the combined organic layers were dried $\left(\mathrm{Na}_{2} \mathrm{SO}_{4}\right)$ and concentrated in vacuo. Flash column chromatography (silica gel, hexanes:EtOAc 5:2) afforded alcohol 17 (523 mg, 83\%) as a viscous yellow oil. 17: $R_{\mathrm{f}}=0.23$ (silica gel, hexanes:EtOAc 4:1); $[\alpha]_{\mathrm{D}}{ }^{25}=-19.7\left(\mathrm{CHCl}_{3}, c=2.0\right.$ ); IR (film) $v_{\max } 3511,2926,1777,1681,1391,1351,1278,1214,771 \mathrm{~cm}^{-1} ;{ }^{1} \mathrm{H}$ NMR $(600 \mathrm{MHz}$, $\left.\mathrm{CDCl}_{3}\right): \delta=7.34(\mathrm{~m}, 2 \mathrm{H}), 7.30(\mathrm{~m}, 1 \mathrm{H}), 7.20(\mathrm{~m}, 2 \mathrm{H}), 6.04(\mathrm{~d}, J=0.9 \mathrm{~Hz}, 1 \mathrm{H}), 5.82(\mathrm{dq}, J=$ 10.4, $1.3 \mathrm{~Hz}, 1 \mathrm{H}), 4.80$ (dddd, $J=9.0,8.8,6.4,3.4 \mathrm{~Hz}, 1 \mathrm{H}), 4.30$ (t, $J=8.8 \mathrm{~Hz}, 1 \mathrm{H}), 4.18$ (dd, $J=8.8,6.4 \mathrm{~Hz}, 1 \mathrm{H}), 3.56(\mathrm{ddd}, J=10.1,6.8,3.4 \mathrm{~Hz}, 1 \mathrm{H}), 3.30(\mathrm{dd}, J=13.6,3.4 \mathrm{~Hz}, 1 \mathrm{H}), 2.89$ 
$(\mathrm{dd}, J=13.6,9.0 \mathrm{~Hz}, 1 \mathrm{H}), 2.59(\mathrm{ddq}, J=10.4,10.1,6.6 \mathrm{~Hz}, 1 \mathrm{H}), 2.48(\mathrm{dd}, J=13.8,2.5 \mathrm{~Hz}, 1$ H), $2.41(\mathrm{dd}, J=13.8,9.0 \mathrm{~Hz}, 1 \mathrm{H}), 1.96(\mathrm{~d}, J=1.5 \mathrm{~Hz}, 3 \mathrm{H}), 1.91(\mathrm{~d}, J=1.0 \mathrm{~Hz}, 3 \mathrm{H}), 1.06$ $\operatorname{ppm}(\mathrm{d}, J=6.6 \mathrm{~Hz}, 3 \mathrm{H}) ;{ }^{13} \mathrm{C} \mathrm{NMR}\left(150 \mathrm{MHz}, \mathrm{CDCl}_{3}\right): \delta=171.5,154.0,145.1,141.2,134.8$, $131.4,129.5 \times 2,129.0 \times 2,127.5,77.2,73.2,66.4,55.1,44.0,39.6,37.5,24.2,16.4,13.9 \mathrm{ppm}$ HRMS (ESI): calcd for $\mathrm{C}_{21} \mathrm{H}_{26} \mathrm{INO}_{4} \mathrm{Na}^{+}\left[\mathrm{M}+\mathrm{Na}^{+}\right]$506.0799, found 506.0795.

Diol 19-epi-14: To a solution of imide $17(6.7 \mathrm{~g}, 13.9 \mathrm{mmol})$ in $\mathrm{Et}_{2} \mathrm{O}(100 \mathrm{~mL})$ were added $\mathrm{LiBH}_{4}(0.458 \mathrm{~g}, 20.8 \mathrm{mmol})$ and $\mathrm{MeOH}(5 \mathrm{~mL})$ at $0{ }^{\circ} \mathrm{C}$. The reaction mixture was stirred for $4 \mathrm{~h}$

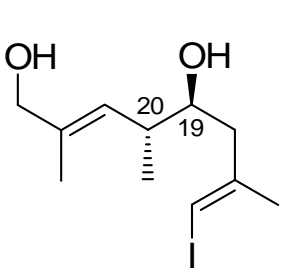

19-epi-14 before it was quenched with $\mathrm{HCl}(1.0 \mathrm{~N}$ aq., $20 \mathrm{~mL})$. The resulting mixture was extracted with EtOAc $(3 \times 50 \mathrm{~mL})$, and the combined organic layers were washed with brine $(20 \mathrm{~mL})$, dried $\left(\mathrm{Na}_{2} \mathrm{SO}_{4}\right)$ and concentrated in vacuo. Flash column chromatography (silica gel, hexanes:EtOAc 4:1) afforded diol 19-epi-14 (4.3 g, 93\%) as a viscous oil. 19-epi-14: $R_{\mathrm{f}}=0.23$ (silica gel, hexanes:EtOAc 3:2); $[\alpha]_{\mathrm{D}}^{25}=-30.7\left(\mathrm{CHCl}_{3}, c=1.0\right)$; IR (film) $v_{\max } 3390,2919,2850,1712,1640,1456,1377,1259$, $1145 \mathrm{~cm}^{-1} ;{ }^{1} \mathrm{H}$ NMR $\left(600 \mathrm{MHz}, \mathrm{CDCl}_{3}\right): \delta=6.02(\mathrm{q}, J=1.2 \mathrm{~Hz}, 1 \mathrm{H}), 5.36(\mathrm{~d}, J=11.2 \mathrm{~Hz}, 1 \mathrm{H})$, 4.05 (s, $2 \mathrm{H}), 3.60$ (ddd, $J=9.1,5.4,3.5 \mathrm{~Hz}, 1 \mathrm{H}), 2.51-2.47$ (m, $1 \mathrm{H}), 2.00(\mathrm{dd}, J=13.9,3.4 \mathrm{~Hz}$, $1 \mathrm{H}), 2.32$ (dd, $J=13.9,9.4 \mathrm{~Hz}, 1 \mathrm{H}), 1.90(\mathrm{~s}, 3 \mathrm{H}), 1.69$ (d, $J=1.4 \mathrm{~Hz}, 3 \mathrm{H}), 1.30 \mathrm{ppm}(\mathrm{d}, J=$ $6.8 \mathrm{~Hz}, 3 \mathrm{H}) ;{ }^{13} \mathrm{C}$ NMR $\left(150 \mathrm{MHz}, \mathrm{CDCl}_{3}\right): \delta=145.2,136.5,126.6,77.1,73.0,68.3,44.8,37.9$, 24.1, 17.4, 14.2 ppm; HRMS (ESI): calcd for $\mathrm{C}_{11} \mathrm{H}_{19} \mathrm{IO}_{2} \mathrm{Na}^{+}\left[\mathrm{M}+\mathrm{Na}^{+}\right]$333.0321, found 333.0322.

TBS ether 19-epi-2: To a solution of diol 19-epi-14 (3.9 g, $12.6 \mathrm{mmol})$ in $\mathrm{CH}_{2} \mathrm{Cl}_{2}(50.0 \mathrm{~mL})$ were added $\mathrm{Et}_{3} \mathrm{~N}(2.63 \mathrm{~mL}, 18.9 \mathrm{mmol}), \mathrm{TBSCl}(2.27 \mathrm{~g}, 15.1 \mathrm{mmol})$ and 4-DMAP (308 mg, 2.5 


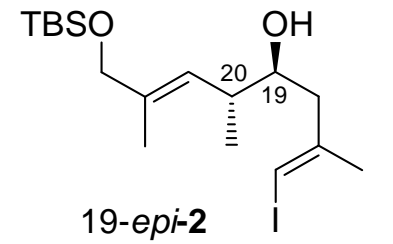

mmol) at $0{ }^{\circ} \mathrm{C}$. The reaction mixture was warmed to room temperature and stirred for $2 \mathrm{~h}$ before it was quenched with water $(10 \mathrm{~mL})$. The resulting mixture was extracted with EtOAc $(3 \times 100 \mathrm{~mL})$, and the combined organic layers were dried $\left(\mathrm{Na}_{2} \mathrm{SO}_{4}\right)$ and concentrated in vacuo. Flash column chromatography (silica gel, hexanes:EtOAc 10:1) afforded TBS ether 19-epi-2 (5.0 g, 92\%) as a colorless oil. 19-epi-2: $R_{\mathrm{f}}=0.33$ (silica gel, hexanes:EtOAc 8:1); $[\alpha]_{\mathrm{D}}{ }^{25}=-15.0\left(\mathrm{CH}_{2} \mathrm{Cl}_{2}, c=\right.$ 0.60); IR (film) $v_{\max } 3455,2956,2929,1462,1256,1071 \mathrm{~cm}^{-1} ;{ }^{1} \mathrm{H} \mathrm{NMR}\left(600 \mathrm{MHz}, \mathrm{CDCl}_{3}\right): \delta=$ 6.00 (s, $1 \mathrm{H}), 5.31(\mathrm{~d}, J=10.2 \mathrm{~Hz}, 1 \mathrm{H}), 4.04$ (s, $2 \mathrm{H}), 3.57$ (ddd, $J=9.0,6.0,3.0 \mathrm{~Hz}, 1 \mathrm{H}), 2.49$ (dqd, $J=10.2,6.6,6.0 \mathrm{~Hz}, 1 \mathrm{H}), 2.40(\mathrm{dd}, J=14.4,2.4 \mathrm{~Hz}, 1 \mathrm{H}), 2.31(\mathrm{dd}, J=13.8,9.0 \mathrm{~Hz}, 1 \mathrm{H})$, $1.90(\mathrm{~s}, 3 \mathrm{H}), 1.63(\mathrm{~s}, 3 \mathrm{H}), 1.01(\mathrm{~d}, J=6.6 \mathrm{~Hz}, 3 \mathrm{H}), 0.93(\mathrm{~s}, 9 \mathrm{H}), 0.08 \mathrm{ppm}(\mathrm{s}, 6 \mathrm{H}) ;{ }^{13} \mathrm{C} \mathrm{NMR}$ $\left(150 \mathrm{MHz}, \mathrm{CDCl}_{3}\right): \delta=145.3,137.0,125.1,76.8,72.8,68.3,44.4,37.9,25.9,24.1,18.4,17.0$ 13.9, -5.2 ppm; HRMS (ESI): calcd for $\mathrm{C}_{17} \mathrm{H}_{33} \mathrm{IO}_{2} \mathrm{SiNa}^{+}\left[\mathrm{M}+\mathrm{Na}^{+}\right]$447.1187, found 447.1188.

Ketone 18: To a solution of alcohol 19-epi-2 (4.8 g, $11.3 \mathrm{mmol})$ in $\mathrm{CH}_{2} \mathrm{Cl}_{2}(20 \mathrm{~mL})$ were added $\mathrm{NaHCO}_{3}(4.75 \mathrm{~g}, 56.5 \mathrm{mmol})$ and DMP $(5.75 \mathrm{~g}, 13.6 \mathrm{mmol})$ at room temperature. The reaction $\mathrm{Na}_{2} \mathrm{~S}_{2} \mathrm{O}_{3}$ (sat. aq.), $\mathrm{NaHCO}_{3}$ (sat. aq.) and water $(1: 1: 1,30 \mathrm{~mL}$ ). The
resulting mixture was diluted with $\mathrm{CH}_{2} \mathrm{Cl}_{2}(40 \mathrm{~mL}$ ), extracted with $\mathrm{CH}_{2} \mathrm{Cl}_{2}(2 \times 50 \mathrm{~mL})$, and the combined organic layers were washed with brine $(20 \mathrm{~mL})$, dried $\left(\mathrm{Na}_{2} \mathrm{SO}_{4}\right)$ and concentrated in vacuo. Flash column chromatography (silica gel, hexanes:EtOAc 10:1) afforded ketone $18(4.5 \mathrm{~g}, 93 \%)$ as a colorless oil. 18: $R_{\mathrm{f}}=0.45$ (silica gel, hexanes:EtOAc 95:5); $[\alpha]_{\mathrm{D}}{ }^{25}=-132.8\left(\mathrm{CHCl}_{3, c}=1.06\right) ; \mathrm{IR}($ film $) v_{\max } 2929,2856,1716,1471,1374,1114,836$ $\mathrm{cm}^{-1} ;{ }^{1} \mathrm{H}$ NMR $\left(600 \mathrm{MHz}, \mathrm{CDCl}_{3}\right): \delta=6.05(\mathrm{q}, J=1.1 \mathrm{~Hz}, 1 \mathrm{H}), 5.30(\mathrm{dq}, J=9.9,1.6 \mathrm{~Hz}, 1 \mathrm{H})$, 
$4.04(\mathrm{~d}, J=0.6 \mathrm{~Hz}, 2 \mathrm{H}), 3.47$ (dq, $J=9.9,6.5 \mathrm{~Hz}, 1 \mathrm{H}), 3.31(\mathrm{~d}, J=0.9 \mathrm{~Hz}, 2 \mathrm{H}), 1.83$ ( d, $J=$ $1.1 \mathrm{~Hz}, 3 \mathrm{H}), 1.69$ (d, $J=1.3 \mathrm{~Hz}, 3 \mathrm{H}), 1.14$ (d, $J=6.5,3 \mathrm{H}), 0.92(\mathrm{~s}, 9 \mathrm{H}), 0.07 \mathrm{ppm}(\mathrm{s}, 6 \mathrm{H})$; ${ }^{13} \mathrm{C}$ NMR $\left(150 \mathrm{MHz}, \mathrm{CDCl}_{3}\right): \delta=207.8,141.4,137.9,122.8,79.0,67.8,50.4,45.3,25.9,24.1$, 18.4, 16.2, 13.9, -5.3 ppm; HRMS (ESI): calcd for $\mathrm{C}_{17} \mathrm{H}_{31} \mathrm{IO}_{2} \mathrm{SiNa}^{+}\left[\mathrm{M}+\mathrm{Na}^{+}\right] 445.1148$, found 445.1139.

Diol 14: To a solution of ketone 18 (4.3 g, $9.7 \mathrm{mmol})$ in $\mathrm{Et}_{2} \mathrm{O}(60 \mathrm{~mL})$ was added Lil $(6.5 \mathrm{~g}, 48.3$ mmol) followed by $\mathrm{Li}(t-\mathrm{BuO}){ }_{3} \mathrm{AlH}(1.0 \mathrm{M}$ in THF, $29.1 \mathrm{~mL}, 29.1 \mathrm{mmol})$ at $-78{ }^{\circ} \mathrm{C}$. The reaction mixture was warmed to room temperature and stirred for $1 \mathrm{~h}$ before it was
mixture was diluted with EtOAc $(100 \mathrm{~mL})$, extracted with EtOAc $(2 \times 50$
$\mathrm{mL})$, and the combined organic layers were washed with brine $(10 \mathrm{~mL})$, dried $\left(\mathrm{Na}_{2} \mathrm{SO}_{4}\right)$, and concentrated in vacuo. To the solution of crude residue in THF (30 mL) was added TBAF (1.0 M in THF, $15.2 \mathrm{~mL}, 15.2 \mathrm{mmol})$ at room temperature. The reaction mixture was stirred for $1 \mathrm{~h}$ before it was concentrated in vacuo. The crude residue was diluted with water $(5 \mathrm{~mL})$, extracted with EtOAc $(3 \times 50 \mathrm{~mL})$, and the combined organic layers were washed with brine $(10 \mathrm{~mL})$, dried $\left(\mathrm{Na}_{2} \mathrm{SO}_{4}\right)$ and concentrated in vacuo. Flash column chromatography (silica gel, hexanes:EtOAc 4:1) afforded diols 14 (2.04 g, 65\%) and 19-epi-14 (0.68 g, 22\%) as viscous oils. All physical properties for diol $\mathbf{1 4}$ and 19-epi-14 were identical to those obtained from TBS ether 13 and imide 17, respectively.

Alcohol 21: To a solution of methoxymethyl allyl ether $(7.95 \mathrm{~g}, 78.0 \mathrm{mmol})$ in THF $(57 \mathrm{~mL})$ was added $s$-BuLi $(1.2 \mathrm{M}$ in cyclohexane, $50.0 \mathrm{~mL}, 60.0 \mathrm{mmol})$ at $-78{ }^{\circ} \mathrm{C}$. The reaction mixture 


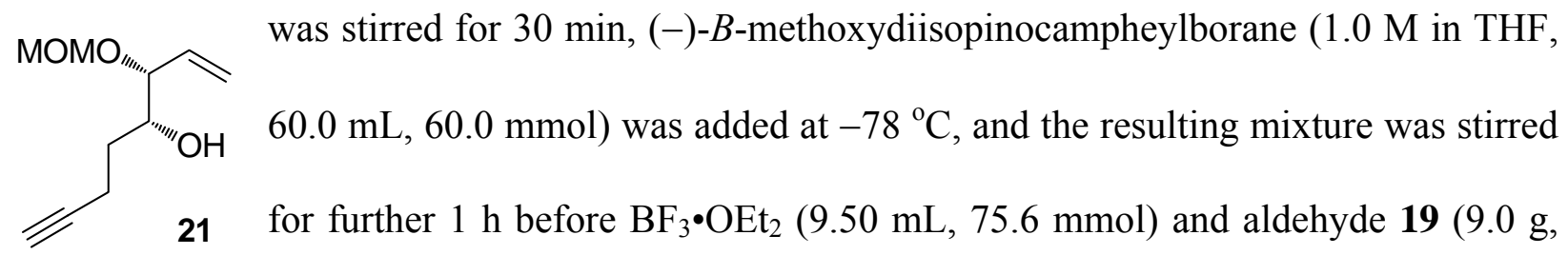

$58.4 \mathrm{mmol}$ ) were added. The reaction mixture was stirred for $3 \mathrm{~h}$, warmed to room temperature and stirred for further $12 \mathrm{~h}$ before it was quenched with $\mathrm{H}_{2} \mathrm{O}_{2}\left(30 \%\right.$ aq.)/ $\mathrm{NaHCO}_{3}$ (sat. aq.) (1:2, $120 \mathrm{~mL})$. The resulting mixture was stirred at room temperature for $1 \mathrm{~h}$, extracted with EtOAc (3 $\times 60 \mathrm{~mL})$, and the combined organic layers were dried $\left(\mathrm{Na}_{2} \mathrm{SO}_{4}\right)$ and concentrated in vacuo.

To a solution of the crude residue in $\mathrm{MeOH}(100 \mathrm{~mL})$ was added $\mathrm{K}_{2} \mathrm{CO}_{3}(25.0 \mathrm{~g}, 181 \mathrm{mmol})$ at room temperature. The reaction mixture was stirred for $7 \mathrm{~h}$ before it was concentrated in vacuo. The crude residue was diluted with EtOAc $(200 \mathrm{~mL})$ and water $(50 \mathrm{~mL})$, extracted with EtOAc $(3 \times 30 \mathrm{~mL})$, and the combined organic layers were dried $\left(\mathrm{Na}_{2} \mathrm{SO}_{4}\right)$ and concentrated in vacuo. Flash column chromatography (silica gel, hexanes:EtOAc 8:1 $\rightarrow$ 2:1) afforded alcohol 21 (8.40 g, $78 \%$ for two steps) ( $>90 \%$ ee by Mosher ester analysis) as a colorless oil. $21: R_{\mathrm{f}}=0.23$ (silica gel, hexanes:EtOAc 5:1); $[\alpha]_{\mathrm{D}}{ }^{25}=-3.6\left(\mathrm{CHCl}_{3}, c=0.44\right)$; IR (film) $v_{\max } 3428,2937,2869,1643$, 1440, 1218, 1150, 1111, 1043, $919 \mathrm{~cm}^{-1} ;{ }^{1} \mathrm{H}$ NMR (600 MHz, $\left.\mathrm{CDCl}_{3}\right): \delta=5.71(\mathrm{ddd}, J=17.4$, 11.4, $7.8 \mathrm{~Hz}, 1 \mathrm{H}), 5.36$ (brd, $J=11.4 \mathrm{~Hz}, 1 \mathrm{H}), 5.34$ (brd, $J=17.4 \mathrm{~Hz}, 1 \mathrm{H}), 4.75$ (d, $J=7.2 \mathrm{~Hz}$, $1 \mathrm{H}), 4.60(\mathrm{~d}, J=6.6 \mathrm{~Hz}, 1 \mathrm{H}), 3.88(\mathrm{t}, J=7.5 \mathrm{~Hz}, 1 \mathrm{H}), 3.70(\mathrm{ddd}, J=10.2,7.2,3.0 \mathrm{~Hz}, 1 \mathrm{H})$, 3.41 (s, $3 \mathrm{H}), 2.50$ (brs, $1 \mathrm{H}), 2.43-2.34(\mathrm{~m}, 2 \mathrm{H}), 1.96$ (t, $J=2.4 \mathrm{~Hz}, 1 \mathrm{H}), 1.76$ (dddd, $J=13.8$, 10.8, 6.5, 3.0 Hz, $1 \mathrm{H}), 1.64 \mathrm{ppm}(\mathrm{dddd}, J=13.2,9.6,7.2,5.4 \mathrm{~Hz}, 1 \mathrm{H}) ;{ }^{13} \mathrm{C} \mathrm{NMR}(150 \mathrm{MHz}$, $\left.\mathrm{CDCl}_{3}\right): \delta=134.6,119.9,93.8,84.0,81.0,71.8,68.5,55.5,31.5,14.7$ ppm; HRMS (ESI): calcd for $\mathrm{C}_{10} \mathrm{H}_{16} \mathrm{O}_{3} \mathrm{Na}^{+}\left[\mathrm{M}+\mathrm{Na}^{+}\right]$207.0991, found 207.0994. 
Carbamate 22: To a solution of alcohol $21(1.5 \mathrm{~g}, 8.15 \mathrm{mmol})$ in $\mathrm{CH}_{2} \mathrm{Cl}_{2}(20 \mathrm{~mL})$ was added $\mathrm{Cl}_{3} \mathrm{CC}(\mathrm{O}) \mathrm{NCO}(3.90 \mathrm{~mL}, 24.5 \mathrm{mmol})$ at room temperature. The reaction mixture was stirred for $1 \mathrm{~h}$ before it was concentrated in vacuo. To a solution of crude residue in
22 The crude residue was diluted with water $(50 \mathrm{~mL})$, extracted with EtOAc $(3 \times 50 \mathrm{~mL})$, and the combined organic layers were washed with water $(2 \times 10 \mathrm{~mL})$, brine $(10 \mathrm{~mL})$, dried $\left(\mathrm{Na}_{2} \mathrm{SO}_{4}\right)$ and concentrated in vacuo. Flash column chromatography (silica gel, hexanes:EtOAc 3:2) afforded carbamate $22(1.85 \mathrm{~g}, 100 \%)$ as a colorless oil. 22: $R_{\mathrm{f}}=0.30$ (silica gel, hexanes:EtOAc 3:2); $[\alpha]_{\mathrm{D}}^{25}=-43.1\left(\mathrm{CHCl}_{3}, c=0.76\right)$; IR (film) $v_{\max } 3456,3360,3295,2955,2893,2826,1718$, 1603, 1387, 1321, 1218, 1151, 1101, 1028, $919 \mathrm{~cm}^{-1} ;{ }^{1} \mathrm{H}$ NMR $\left(600 \mathrm{MHz}, \mathrm{CDCl}_{3}\right): \delta=5.73$ (ddd, $J=17.4,10.2,7.2 \mathrm{~Hz}, 1 \mathrm{H}), 5.33$ (brd, $J=17.4 \mathrm{~Hz}, 1 \mathrm{H}), 5.32$ (brd, $J=10.8 \mathrm{~Hz}, 1 \mathrm{H}), 4.98$ $(\mathrm{dt}, J=9.0,4.5 \mathrm{~Hz}, 1 \mathrm{H}), 4.74(\mathrm{brs}, 2 \mathrm{H}), 4.70(\mathrm{~d}, J=6.6 \mathrm{~Hz}, 1 \mathrm{H}), 4.59(\mathrm{~d}, J=6.6 \mathrm{~Hz}, 1 \mathrm{H})$, $4.15(\mathrm{t}, J=6.0 \mathrm{~Hz}, 1 \mathrm{H}), 3.40(\mathrm{~s}, 3 \mathrm{H}), 2.32-2.27(\mathrm{~m}, 2 \mathrm{H}), 1.97(\mathrm{t}, J=2.4 \mathrm{~Hz}, 1 \mathrm{H}), 1.95-1.91$ ppm (m, $2 \mathrm{H}) ;{ }^{13} \mathrm{C} \mathrm{NMR}\left(150 \mathrm{MHz}, \mathrm{CDCl}_{3}\right): \delta=156.3,133.7,119.6,94.1,83.3,77.7,74.6,68.7$, 55.7, 29.7, 14.8 ppm; HRMS (ESI): calcd for $\mathrm{C}_{11} \mathrm{H}_{17} \mathrm{NO}_{4} \mathrm{Na}^{+}\left[\mathrm{M}+\mathrm{Na}^{+}\right] 250.1049$, found 250.1060 .

Bromide 23: To a solution of alkyne $22(6.84 \mathrm{~g}, 30.0 \mathrm{mmol})$ in acetone $(100 \mathrm{~mL})$ were added NBS $(5.86 \mathrm{~g}, 33.0 \mathrm{mmol})$ and $\mathrm{AgNO}_{3}(510 \mathrm{mg}, 3.0 \mathrm{mmol})$ at room temperature. The reaction mixture was stirred for $1 \mathrm{~h}$ before it was quenched with $\mathrm{NaHCO}_{3}(30$ 
were dried $\left(\mathrm{Na}_{2} \mathrm{SO}_{4}\right)$ and concentrated in vacuo. Flash column chromatography (silica gel, hexanes:EtOAc 3:2) afforded bromide $23(8.32 \mathrm{~g}, 90 \%)$ as a colorless oil. 23: $R_{\mathrm{f}}=0.40$ (silica gel, hexanes:EtOAc 3:2); $[\alpha]_{\mathrm{D}}^{25}=-25.0\left(\mathrm{CHCl}_{3}, c=0.49\right)$; IR (film) $v_{\max } 3456,3351,2953$, $1716,1602,1521,1387,1321,1217,1151,1100,1028,918 \mathrm{~cm}^{-1} ;{ }^{1} \mathrm{H}$ NMR $\left(600 \mathrm{MHz}, \mathrm{CDCl}_{3}\right)$ : $\delta=5.73(\mathrm{ddd}, J=18.0,10.8,7.8 \mathrm{~Hz}, 1 \mathrm{H}), 5.34(\mathrm{brd}, J=18.0 \mathrm{~Hz}, 1 \mathrm{H}), 5.33($ brd, $J=8.4 \mathrm{~Hz}, 1$ H), 4.95-4.92 (m, $1 \mathrm{H}), 4.75$ (brs, $2 \mathrm{H}), 4.70$ (d, $J=7.2 \mathrm{~Hz}, 1 \mathrm{H}), 4.59$ (d, $J=6.6 \mathrm{~Hz}, 1 \mathrm{H}), 4.13$ $(\mathrm{t}, J=6.0 \mathrm{~Hz}, 1 \mathrm{H}), 3.40(\mathrm{~s}, 3 \mathrm{H}), 2.34(\mathrm{~m}, 2 \mathrm{H}), 1.93-1.84 \mathrm{ppm}(\mathrm{m}, 2 \mathrm{H}) ;{ }^{13} \mathrm{C} \mathrm{NMR}(150 \mathrm{MHz}$, $\left.\mathrm{CDCl}_{3}\right): \delta=156.3,133.7,119.7,94.1,79.1,77.7,74.7,55.7,38.4,29.5,16.2$ ppm; HRMS (ESI): calcd for $\mathrm{C}_{11} \mathrm{H}_{16} \mathrm{BrNO}_{4} \mathrm{Na}^{+}\left[\mathrm{M}+\mathrm{Na}^{+}\right]$: 328.0155; found 328.0152 .

Vinyl stannane 3: To a solution bromide $23(800 \mathrm{mg}, 2.59 \mathrm{mmol})$ in THF $(20 \mathrm{~mL})$ were added $\mathrm{Pd}(\mathrm{dba})_{2}(75 \mathrm{mg}, 0.129 \mathrm{mmol})$ and $\mathrm{PPh}_{3}(136 \mathrm{mg}, 0.59 \mathrm{mmol})$ at room temperature. The reaction mixture was stirred for $10 \mathrm{~min}$ before $n-\mathrm{Bu}_{3} \mathrm{SnH}(1.54 \mathrm{~mL}, 5.71 \mathrm{mmol})$ was added over a period of $1 \mathrm{~h}$ via a syringe pump and then concentrated in vacuo. Flash column chromatography (silica gel, hexanes:EtOAc:Et $3 \mathrm{~N}$ 4:1:0.05) afforded vinyl stannane 3 (1.04 g, 77\%) as a colorless oil. 3: $R_{\mathrm{f}}=0.30$ (silica gel, hexanes:EtOAc 5:2); $[\alpha]_{\mathrm{D}}{ }^{25}=-26.8\left(\mathrm{CHCl}_{3}, c=1.5\right.$ ); IR (film) $v_{\max } 3360,2953,2926,2856,1720,1599,1464,1377,1322,1275,1152,1031,922$, $750 \mathrm{~cm}^{-1} ;{ }^{1} \mathrm{H}$ NMR $\left(600 \mathrm{MHz}, \mathrm{CDCl}_{3}\right): \delta=5.96-5.85(\mathrm{~m}, 2 \mathrm{H}), 5.74(\mathrm{ddd}, J=17.4,10.8,7.8 \mathrm{~Hz}$, $1 \mathrm{H}), 5.34$ (brd, $J=11.4 \mathrm{~Hz}, 1 \mathrm{H}), 5.33(\mathrm{brd}, J=17.4 \mathrm{~Hz}, 1 \mathrm{H}), 4.89$ (dt, $J=8.4,4.8 \mathrm{~Hz}, 1 \mathrm{H})$, 4.72 (brs, $2 \mathrm{H}), 4.71$ (d, $J=6.6 \mathrm{~Hz}, 1 \mathrm{H}), 4.60(\mathrm{~d}, J=6.6 \mathrm{~Hz}, 1 \mathrm{H}), 4.14(\mathrm{dd}, J=7.2,5.4 \mathrm{~Hz}, 1$ H), 3.40 (s, 3 H), 2.27-2.18 (m, 2 H), 1.79-1.69 (m, 2 H), 1.49 (q, $J=7.2 \mathrm{~Hz}, 6 \mathrm{H}), 1.32$ (sext, $J$ $=7.2 \mathrm{~Hz}, 6 \mathrm{H}), 0.90(\mathrm{t}, J=7.2 \mathrm{~Hz}, 9 \mathrm{H}), 0.87 \mathrm{ppm}(\mathrm{t}, J=7.8 \mathrm{~Hz}, 6 \mathrm{H}) ;{ }^{13} \mathrm{C}$ NMR $(150 \mathrm{MHz}$, 
$\left.\mathrm{CDCl}_{3}\right): \delta=156.5,148.0,134.0,128.0,119.3,94.0,77.7,75.5,55.7,33.5,30.0,29.0,27.2,13.7$, 9.4 ppm; HRMS (ESI): calcd for $\mathrm{C}_{23} \mathrm{H}_{45} \mathrm{NO}_{4} \mathrm{SnNa}^{+}\left[\mathrm{M}+\mathrm{Na}^{+}\right]$542.2262, found 542.2271.

Diol 25 and epoxide 26: To a solution of olefin $24(8.34 \mathrm{~g}, 38.92 \mathrm{mmol})$ in $\mathrm{CH}_{2} \mathrm{Cl}_{2}(140 \mathrm{~mL})$ was added $\mathrm{NaHCO}_{3}(6.54 \mathrm{~g}, 77.85 \mathrm{mmol})$ followed by $m$-CPBA $(8.73 \mathrm{~g}, 50.6 \mathrm{mmol})$ at $0{ }^{\circ} \mathrm{C}$.
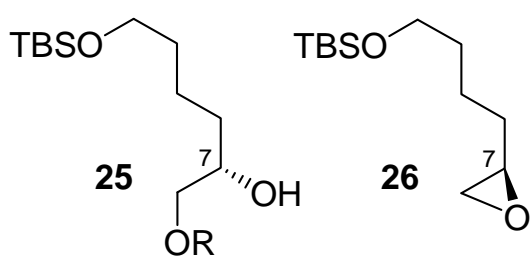

The reaction mixture was warmed to room temperature and stirred for $2 \mathrm{~h}$ before it was diluted with $\mathrm{CH}_{2} \mathrm{Cl}_{2}(60 \mathrm{~mL})$. The resulting mixture was washed with $\mathrm{NaHCO}_{3}(3 \times 50 \mathrm{~mL}$, sat. aq.) and $\mathrm{H}_{2} \mathrm{O}(2 \times 25 \mathrm{~mL})$, dried $\left(\mathrm{Na}_{2} \mathrm{SO}_{4}\right)$ and concentrated in vacuo. To a solution of $(R, R)$ $N, N^{\prime}$-bis(3,5-di-t-butylsalicylidene)-1,2-cyclohexanediaminato(2-)]cobalt $\quad$ (II) $\quad\left(\begin{array}{llll}0.04 & \mathrm{~g}, & 0.067\end{array}\right.$ mmol) in $\mathrm{CH}_{2} \mathrm{Cl}_{2}(2 \mathrm{~mL})$ was added acetic acid $(7 \mu \mathrm{L}, 0.13 \mathrm{mmol})$ at room temperature. The reaction mixture was stirred in open air for $1 \mathrm{~h}$ before it was concentrated in vacuo and the catalyst was dried thoroughly. To a solution of dry catalyst in crude epoxide ( $3.12 \mathrm{~g}, 13.54 \mathrm{mmol})$ was added $\mathrm{H}_{2} \mathrm{O}(0.134 \mathrm{~mL}, 7.45 \mathrm{mmol})$ over a period of $1 \mathrm{~h}$ at $0{ }^{\circ} \mathrm{C}$. The reaction mixture was warmed to room temperature and stirred for $24 \mathrm{~h}$. Flash column chromatography (silica gel, hexanes:EtOAc 5:1 $\rightarrow$ 1:1) afforded diol $25(1.24 \mathrm{~g}, 43 \%)$ [>90\% ee by measurement of its optical rotation and that of later intermediates ent-26 and ent-27, and Mosher ester ${ }^{1} \mathrm{H}$ NMR (600 MHz) analysis of ent-27] and epoxide 26 (1.46 g, 47\%) [ $>90 \%$ ee by Mosher ester ${ }^{1} \mathrm{H}$ NMR (600 MHz) analysis of 27] as yellow oils. All physical properties for diol 25 and epoxide 26 were identical to those reported in literature. ${ }^{1}$

Alcohol 27: To a solution of $\mathrm{Me}_{3} \mathrm{~S}^{+} \mathrm{I}^{-}(0.89 \mathrm{~g}, 4.35 \mathrm{mmol})$ in THF $(18 \mathrm{~mL})$ was added $n$-BuLi $(1.6 \mathrm{M}$ in hexanes, $3.94 \mathrm{~mL}, 6.31 \mathrm{mmol})$ at $-30{ }^{\circ} \mathrm{C}$. The reaction mixture was stirred for $30 \mathrm{~min}$ 


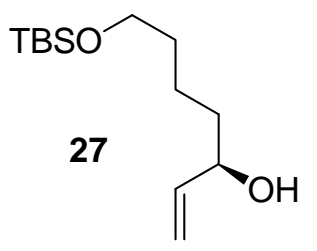

before a solution of epoxide $26(0.25 \mathrm{~g}, 1.08 \mathrm{mmol})$ in THF $(3 \mathrm{~mL})$ was added slowly. The reaction mixture was stirred at $-30{ }^{\circ} \mathrm{C}$ for $1 \mathrm{~h}$ and then warmed to room temperature over a period of $3.5 \mathrm{~h}$ before it was quenched with water $(2 \mathrm{~mL})$. The resulting mixture was diluted with EtOAc $(25 \mathrm{~mL})$, washed with water $(3$ $\times 15 \mathrm{~mL})$, brine $(15 \mathrm{~mL})$, dried $\left(\mathrm{Na}_{2} \mathrm{SO}_{4}\right)$ and concentrated in vacuo. Flash column chromatography (silica gel, hexanes:EtOAc 5:1 $\rightarrow$ 3:1) afforded alcohol $27(0.24 \mathrm{~g}, 90 \%)$ as a yellow oil. 27: $R_{\mathrm{f}}=0.65$ (silica gel, hexanes:EtOAc 1:1); $[\alpha]_{\mathrm{D}}{ }^{25}=-6.0\left(\mathrm{CHCl}_{3}, c=2.2\right)$; IR (film) $v_{\max } 3387,2930,2858,1472,1462,1388,1361,1255,1100,991,920,836,774 \mathrm{~cm}^{-1} ;{ }^{1} \mathrm{H}$ NMR $\left(600 \mathrm{MHz}, \mathrm{CDCl}_{3}\right): \delta=5.85$ (ddd, $\left.J=16.2,9.6,6.0 \mathrm{~Hz}, 1 \mathrm{H}\right), 5.21$ (brd, $\left.J=16.8 \mathrm{~Hz}, 1 \mathrm{H}\right), 5.05$ (brd, $J=10.2 \mathrm{~Hz}, 1 \mathrm{H}), 4.10-4.09$ (m, $1 \mathrm{H}), 3.62-3.60$ (m, $2 \mathrm{H}), 1.89$ (m, $1 \mathrm{H}), 1.59-1.51$ (m, 2 H), 1.49-1.34 (m, $4 \mathrm{H}), 0.90$ (s, $9 \mathrm{H}), 0.06 \mathrm{ppm}(\mathrm{s}, 6 \mathrm{H}) ;{ }^{13} \mathrm{C} \mathrm{NMR}\left(150 \mathrm{MHz}, \mathrm{CDCl}_{3}\right): \delta=141.2$, 114.5, 73.1, 63.0, 36.7, 32.6, 25.9, 21.6, 18.3, -5.3 ppm; HRMS (ESI): calcd for $\mathrm{C}_{13} \mathrm{H}_{28} \mathrm{O}_{2} \mathrm{SiNa}^{+}$ $\left[\mathrm{M}+\mathrm{Na}^{+}\right] 267.1750$, found 267.1760.

MOM ether 28: To a solution of alcohol $27(1.67 \mathrm{~g}, 6.85 \mathrm{mmol})$ in $\mathrm{CH}_{2} \mathrm{Cl}_{2}(17 \mathrm{~mL})$ were added $i-\operatorname{Pr}_{2} \mathrm{NEt}(2.44 \mathrm{~mL}, 13.7 \mathrm{mmol})$ and $\mathrm{MOMCl}(2.08 \mathrm{~mL}, 27.4 \mathrm{mmol})$ at $0{ }^{\circ} \mathrm{C}$. The reaction mixture was stirred for 30 min then warmed to room temperature and stirred for

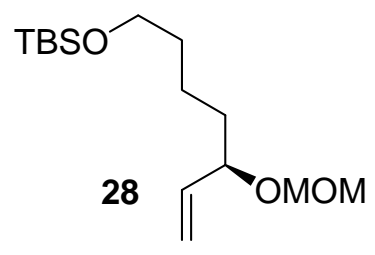
$5.5 \mathrm{~h}$ before it was diluted with $\mathrm{CH}_{2} \mathrm{Cl}_{2}(15 \mathrm{~mL})$. The resulting mixture was washed with water $(3 \times 15 \mathrm{~mL})$, brine $(15 \mathrm{~mL})$, dried $\left(\mathrm{Na}_{2} \mathrm{SO}_{4}\right)$ and concentrated in vacuo. Flash column chromatography (silica gel, hexanes:EtOAc 5:1 $\rightarrow$ 4:1) afforded MOM ether $28(1.6 \mathrm{~g}, 85 \%)$ as a yellow oil. 28: $R_{\mathrm{f}}=0.80$ (silica gel, hexanes:EtOAc, 4:1); $[\alpha]_{\mathrm{D}}{ }^{25}=+50.0\left(\mathrm{CHCl}_{3}, c=2.5\right)$; IR (film) $v_{\max } 2929,1886$, $2858,1472,1463,1388,1361,1254,1218,1152,1097,1035,1006,992,920,835,774,662,619$, 
$577 \mathrm{~cm}^{-1} ;{ }^{1} \mathrm{H} \mathrm{NMR}\left(600 \mathrm{MHz}, \mathrm{CDCl}_{3}\right): \delta=5.67(\mathrm{ddd}, J=17.4,10.2,7.8 \mathrm{~Hz}, 1 \mathrm{H}), 5.22-5.19(\mathrm{~m}$, $2 \mathrm{H}), 4.71(\mathrm{~d}, J=6.6 \mathrm{~Hz}, 1 \mathrm{H}), 4.54(\mathrm{~d}, J=6.6 \mathrm{~Hz}, 1 \mathrm{H}), 4.01-3.97(\mathrm{~m}, 1 \mathrm{H}), 3.63-3.61(\mathrm{~m}, 2 \mathrm{H})$, $3.38(\mathrm{~s}, 3 \mathrm{H}), 1.67-1.56(\mathrm{~m}, 6 \mathrm{H}), 0.90(\mathrm{~s}, 9 \mathrm{H}), 0.05 \mathrm{ppm}(\mathrm{s}, 6 \mathrm{H}) ;{ }^{13} \mathrm{C} \mathrm{NMR}\left(150 \mathrm{MHz}, \mathrm{CDCl}_{3}\right)$ : $\delta=138.4,117.1,93.7,77.3,63.0,55.4,35.1,32.7,25.9,21.7,18.3,-5.2$ ppm; HRMS (ESI): calcd for $\mathrm{C}_{15} \mathrm{H}_{32} \mathrm{O}_{3} \mathrm{SiNa}^{+}\left[\mathrm{M}+\mathrm{Na}^{+}\right]$311.2012, found, 311.2036.

Alcohol 29: To a solution of TBS ether 28 (1.54 g, $5.34 \mathrm{mmol})$ in THF (15 mL) was added TBAF (1.0 M in THF, $6.41 \mathrm{~mL}, 6.41 \mathrm{mmol})$ at room temperature. The reaction mixture was stirred for $2 \mathrm{~h}$ before it was diluted with EtOAc $(20 \mathrm{~mL})$. The resulting
mixture was washed with water $(15 \mathrm{~mL})$, brine $(3 \times 15 \mathrm{~mL})$, and the chromatography (silica gel, hexanes:EtOAc 5:1 $\rightarrow$ 2:1) afforded alcohol $29(0.87 \mathrm{~g}, 95 \%)$ as a pale yellow oil. 29: $R_{\mathrm{f}}=0.25$ (silica gel, hexanes:EtOAc 4:1); $[\alpha]_{\mathrm{D}}{ }^{25}=+86.8\left(\mathrm{CHCl}_{3}, c=0.72\right.$ ); IR (film) $v_{\max } 3426,2937,1424,1219,1097,1150,1034,993,919,650 \mathrm{~cm}^{-1} ;{ }^{1} \mathrm{H}$ NMR (600 $\left.\mathrm{MHz}, \mathrm{CDCl}_{3}\right): \delta=5.68(\mathrm{ddd}, J=18.0,10.2,7.8 \mathrm{~Hz}, 1 \mathrm{H}), 5.22-5.19(\mathrm{~m}, 2 \mathrm{H}), 4.71(\mathrm{~d}, J=6.6$ Hz, $1 \mathrm{H}), 4.55(\mathrm{~d}, J=7.2 \mathrm{~Hz}, 1 \mathrm{H}), 4.02-3.98(\mathrm{~m}, 1 \mathrm{H}), 3.66$ (m, $2 \mathrm{H}), 3.38$ (s, $3 \mathrm{H}), 1.69-1.56$ $\operatorname{ppm}(\mathrm{m}, 6 \mathrm{H}) ;{ }^{13} \mathrm{C} \mathrm{NMR}\left(150 \mathrm{MHz}, \mathrm{CDCl}_{3}\right): \delta=138.3,117.2,93.7,77.3,62.8,55.4,35.1,32.6$, 21.5 ppm; HRMS (ESI): calcd for $\mathrm{C}_{9} \mathrm{H}_{18} \mathrm{O}_{3} \mathrm{Na}^{+}\left[\mathrm{M}+\mathrm{Na}^{+}\right]$174.1256, found 174.1260.

Aldehyde 30: To a solution of alcohol $29(0.2 \mathrm{~g}, 1.14 \mathrm{mmol})$ in $\mathrm{CH}_{2} \mathrm{Cl}_{2}(5 \mathrm{~mL})$ were added $\mathrm{NaHCO}_{3}(0.48 \mathrm{~g}, 5.73 \mathrm{mmol})$ and DMP $(0.67 \mathrm{~g}, 1.58 \mathrm{mmol})$ at room temperature. The reaction mixture was stirred for $2 \mathrm{~h}$ before it was quenched with a mixture of $\mathrm{Na}_{2} \mathrm{~S}_{2} \mathrm{O}_{3}$ (sat. aq.), $\mathrm{NaHCO}_{3}$ (sat. aq.) and $\mathrm{H}_{2} \mathrm{O}(1: 1: 1,12 \mathrm{~mL})$. The resulting mixture was diluted with $\mathrm{CH}_{2} \mathrm{Cl}_{2}(10 \mathrm{~mL})$, 


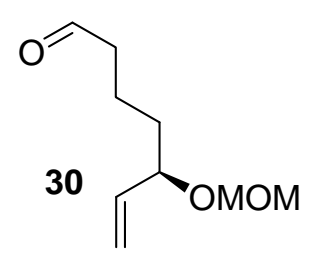

washed with water $(3 \times 15 \mathrm{~mL})$, brine $(15 \mathrm{~mL})$, dried $\left(\mathrm{Na}_{2} \mathrm{SO}_{4}\right)$ and concentrated in vacuo to afford crude aldehyde $30(0.18 \mathrm{~g}, 95 \%)$ as a yellow $R_{\mathrm{f}}=0.75$ (silica gel, hexanes:EtOAc 4:1); $[\alpha]_{\mathrm{D}}{ }^{25}=+50.6\left(\mathrm{CHCl}_{3}, c=0.86\right)$; IR (film) $v_{\max } 2929$, $2718,1725,1462,1410,1215,1150,1097,1034,995,918 \mathrm{~cm}^{-1} ;{ }^{1} \mathrm{H}$ NMR (600 MHz, $\left.\mathrm{CDCl}_{3}\right): \delta$ $=9.78(\mathrm{t}, J=1.2 \mathrm{~Hz}, 1 \mathrm{H}), 5.68(\mathrm{ddd}, J=17.4,10.2,7.8 \mathrm{~Hz}, 1 \mathrm{H}), 4.01(\mathrm{q}, J=6.6 \mathrm{~Hz}, 1 \mathrm{H})$, 5.24-5.20 (m, $2 \mathrm{H}), 4.71(\mathrm{~d}, J=6.6 \mathrm{~Hz}, 1 \mathrm{H}), 4.47$ (d, $J=6.6 \mathrm{~Hz}, 1 \mathrm{H}), 3.38(\mathrm{~s}, 3 \mathrm{H}), 2.49-2.47$ (m, $2 \mathrm{H}), 1.79-1.63 \mathrm{ppm}(\mathrm{m}, 4 \mathrm{H}) ;{ }^{13} \mathrm{C} \mathrm{NMR}\left(150 \mathrm{MHz}, \mathrm{CDCl}_{3}\right): \delta=202.2,137.9,117.5,93.7$, 76.9, 55.5, 43.6, 34.7, 18.0 ppm; HRMS (ESI): calcd for $\mathrm{C}_{9} \mathrm{H}_{16} \mathrm{O}_{3} \mathrm{Na}^{+}\left[\mathrm{M}+\mathrm{Na}^{+}\right]$195.0991, found 195.0984.

Enoate 31: To a solution of aldehyde $30(0.19 \mathrm{~g}, 1.13 \mathrm{mmol})$ in $\mathrm{CH}_{2} \mathrm{Cl}_{2}(4 \mathrm{~mL})$ was added $\mathrm{PPh}_{3}=\mathrm{CH}_{2} \mathrm{CO}_{2} \mathrm{Me}(0.37 \mathrm{~g}, 1.13 \mathrm{mmol})$ at room temperature. The reaction mixture was stirred for

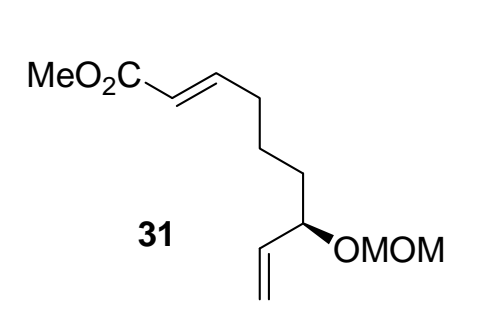

$16 \mathrm{~h}$ before it was concentrated in vacuo. Flash column chromatography (silica gel, hexanes:EtOAc 5:1 $\rightarrow$ 4:1) afforded enoate $31(0.23 \mathrm{~g}, 90 \%)$ as yellow oil. $31: R_{\mathrm{f}}=0.75$ (silica gel, hexanes:EtOAc 4:1); $[\alpha]_{\mathrm{D}}^{25}=+46.8\left(\mathrm{CHCl}_{3}, c=0.52\right)$; IR (film) $v_{\max } 2926,1726,1658,1436,1270,1200,1149,1097,1033,919,774 \mathrm{~cm}^{-1} ;{ }^{1} \mathrm{H}$ NMR $(600 \mathrm{MHz}$, $\left.\mathrm{CDCl}_{3}\right): \delta=6.97(\mathrm{dt}, J=15.6,7.8 \mathrm{~Hz}, 1 \mathrm{H}), 5.84(\mathrm{brd}, J=15.6 \mathrm{~Hz}, 1 \mathrm{H}), 5.66(\mathrm{ddd}, J=17.4,8.4$, $4.2 \mathrm{~Hz}, 1 \mathrm{H}), 5.22-5.19(\mathrm{~m}, 2 \mathrm{H}), 4.71(\mathrm{~d}, J=6.6 \mathrm{~Hz}, 1 \mathrm{H}), 4.53(\mathrm{~d}, J=7.2 \mathrm{~Hz}, 1 \mathrm{H}), 4.00$ (brd, $J$ $=5.4 \mathrm{~Hz}, 1 \mathrm{H}), 3.73(\mathrm{~s}, 3 \mathrm{H}), 3.38(\mathrm{~s}, 3 \mathrm{H}), 2.25-2.24(\mathrm{~m}, 2 \mathrm{H}), 1.67-1.53 \mathrm{ppm}(\mathrm{m}, 4 \mathrm{H}) ;{ }^{13} \mathrm{C}$ $\operatorname{NMR}\left(150 \mathrm{MHz}, \mathrm{CDCl}_{3}\right): \delta=167.1,149.1,138.1,121.1,117.4,93.7,77.2,55.4,51.4,34.8,32.0$, 23.8 ppm; HRMS (ESI): calcd for $\mathrm{C}_{12} \mathrm{H}_{20} \mathrm{O}_{4} \mathrm{Na}^{+}\left[\mathrm{M}+\mathrm{Na}^{+}\right] 251.1253$, found 251.1275. 
Carboxylic acid 4: To a solution of ester $31(0.38 \mathrm{~g}, 1.66 \mathrm{mmol})$ in dioxane $/ \mathrm{H}_{2} \mathrm{O}(4: 1,15 \mathrm{~mL})$ was added $\mathrm{KOH}(0.46 \mathrm{~g}, 8.33 \mathrm{mmol})$ at room temperature. The reaction mixture was stirred for

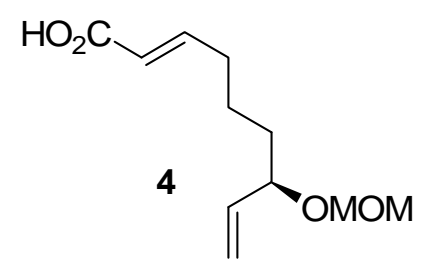
$24 \mathrm{~h}$ before it was diluted with water $(10 \mathrm{~mL})$ and extracted with EtOAc $(2 \times 10 \mathrm{~mL})$. The aqueous layer was separated and carefully acidified with $\mathrm{HCl}(1.0 \mathrm{~N})$ to $\mathrm{pH} \sim 4$, and then extracted with EtOAc $(3 \times 15 \mathrm{~mL})$. The combined organic layers were washed with water $(2 \times 15 \mathrm{~mL})$, dried $\left(\mathrm{Na}_{2} \mathrm{SO}_{4}\right)$ and concentrated in vacuo to afford acid $4(0.3 \mathrm{~g}, 85 \%)$ as a pale yellow oil which was used directly without further purification. 4: $R_{\mathrm{f}}=0.20$ (silica gel, hexanes:EtOAc 4:1); $[\alpha]_{\mathrm{D}}{ }^{25}=+80.2\left(\mathrm{CHCl}_{3}, c=1.5\right)$; IR (film) $v_{\max } 2943,1696,1651,1422$, 1285, 1216, 1149, 1097, 1032, 984, $920 \mathrm{~cm}^{-1} ;{ }^{1} \mathrm{H}$ NMR $\left(600 \mathrm{MHz}, \mathrm{CDCl}_{3}\right): \delta=7.08(\mathrm{dt}, J=$ 15.6, $7.2 \mathrm{~Hz}, 1 \mathrm{H}), 5.84(\mathrm{brd}, J=15.6 \mathrm{~Hz}, 1 \mathrm{H}), 5.67$ (ddd, $J=16.8,10.2,6.6 \mathrm{~Hz}, 1 \mathrm{H})$, 5.23-5.19 (m, $2 \mathrm{H}), 4.71(\mathrm{~d}, J=6.6 \mathrm{~Hz}, 1 \mathrm{H}), 4.54(\mathrm{~d}, J=7.2 \mathrm{~Hz}, 1 \mathrm{H}), 4.01-4.0(\mathrm{~m}, 1 \mathrm{H}), 3.38$ (s, $3 \mathrm{H}), 2.29-2.27$ (m, $2 \mathrm{H}), 1.66-1.57$ (m, $2 \mathrm{H}), 1.57-1.53 \mathrm{ppm}(\mathrm{m}, 2 \mathrm{H}) ;{ }^{13} \mathrm{C}$ NMR (150 MHz, $\left.\mathrm{CDCl}_{3}\right): \delta=171.7,151.7,138.0,121.0,117.5,93.7,77.0,55.5,35.0,32.1,23.7$ ppm; HRMS (ESI): calcd for $\mathrm{C}_{11} \mathrm{H}_{18} \mathrm{O}_{4} \mathrm{Na}^{+}\left[\mathrm{M}+\mathrm{Na}^{+}\right]$237.1103, found 237.1111.

Tosylate 32: To a solution of diol $25(8.56 \mathrm{~g}, 40 \mathrm{mmol})$ in $\mathrm{CH}_{2} \mathrm{Cl}_{2}(80 \mathrm{~mL})$ was added $n-\mathrm{Bu}_{2} \mathrm{SnO}$ $(1.99 \mathrm{~g}, 8.0 \mathrm{mmol})$ followed by $\mathrm{Et}_{3} \mathrm{~N}(5.56 \mathrm{~mL}, 40 \mathrm{mmol})$ at room temperature. The reaction

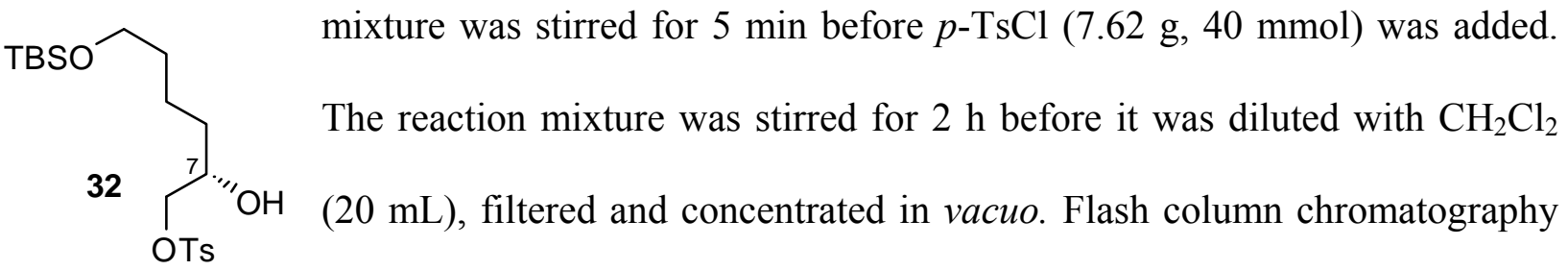
(silica gel, hexanes:EtOAc 5:1 $\rightarrow 2: 1)$ afforded tosylate $32(12.5 \mathrm{~g}, 78 \%)$ as a colorless oil. 32: $R_{\mathrm{f}}$ $=0.35$ (silica gel, hexanes:EtOAc 1:1); $[\alpha]_{\mathrm{D}}^{25}=+4.6\left(\mathrm{CHCl}_{3}, c=0.89\right)$; IR (film) $v_{\max } 3435$, 
2929, 2857, 1598, 1462, 1360, 1254, 1189, 1176, 1096, 970, 843, 813, 775, $666 \mathrm{~cm}^{-1}$; ${ }^{1} \mathrm{H}$ NMR $\left(600 \mathrm{MHz}, \mathrm{CDCl}_{3}\right): \delta=7.81(\mathrm{~d}, J=8.4 \mathrm{~Hz}, 2 \mathrm{H}), 7.37(\mathrm{~d}, J=7.8 \mathrm{~Hz}, 2 \mathrm{H}), 4.04(\mathrm{dd}, J=9.6,7.2$ Hz, $1 \mathrm{H}), 3.89$ (dt, $J=9.6,6.6 \mathrm{~Hz}, 1 \mathrm{H}), 3.86$ (m, $1 \mathrm{H}), 3.60$ (bt, $J=6.0 \mathrm{~Hz}, 2 \mathrm{H}), 2.47$ (s, $3 \mathrm{H})$, 2.13-2.12 (m, $1 \mathrm{H}), 1.52-1.36(\mathrm{~m}, 6 \mathrm{H}), 0.89(\mathrm{~s}, 9 \mathrm{H}), 0.04 \mathrm{ppm}(\mathrm{s}, 6 \mathrm{H}) ;{ }^{13} \mathrm{C}$ NMR $(150 \mathrm{MHz}$ $\left.\mathrm{CDCl}_{3}\right): \delta=145.0,132.8,129.9,127.9,73.0,69.4,62.8,32.4,32.3,25.9,21.6,21.6,18.3,-5.3$ ppm; HRMS (ESI): calcd for $\mathrm{C}_{19} \mathrm{H}_{35} \mathrm{O}_{5} \mathrm{SSi}^{+}\left[\mathrm{M}+\mathrm{H}^{+}\right]$403.1969, found 403.1974 .

Epoxide ent-26: To a solution of tosylate $32(0.59 \mathrm{~g}, 1.47 \mathrm{mmol})$ in $\mathrm{MeOH} / \mathrm{CH}_{2} \mathrm{Cl}_{2}(10: 1,12 \mathrm{~mL})$ was added $\mathrm{K}_{2} \mathrm{CO}_{3}(0.306 \mathrm{~g}, 2.2 \mathrm{mmol})$ at $0{ }^{\circ} \mathrm{C}$. The reaction mixture was warmed to room ent-26 $26 \begin{aligned} & \text { temperature and stirred for } 2 \mathrm{~h} \text { before it was concentrated in vacuo. The } \\ & \text { resulting residue was diluted with EtOAc }(15 \mathrm{~mL}) \text {, washed with brine }(3 \times 15 \\ & \overline{\mathrm{O}} \mathrm{mL}) \text {, dried }\left(\mathrm{Na}_{2} \mathrm{SO}_{4}\right) \text {, and concentrated in vacuo. Flash column chromatography }\end{aligned}$ (silica gel, hexanes:EtOAc 5:1 $\rightarrow$ 4:1) afforded epoxide ent-26 $(0.27 \mathrm{~g}, 82 \%)$ as a yellow oil. All physical properties (except optical rotation in opposite sign) were identical to those reported for epoxide 26 obtained from olefin 24.

Tetraene 33: To a mixture of vinyl iodide 2 (500 mg, $1.18 \mathrm{mmol})$, vinyl stannane 3 (643 mg, $1.41 \mathrm{mmol}), \mathrm{Pd}(\mathrm{dba})_{2}(104 \mathrm{mg}, 0.18 \mathrm{mmol}), \mathrm{AsPh}_{3}(1.08 \mathrm{~g}, 3.54 \mathrm{mmol})$ and $\mathrm{LiCl}(148 \mathrm{mg}, 3.54$ mmol, flame dried) was added NMP (4.0 mL, degassed) at room temperature. The reaction mixture was stirred for $12 \mathrm{~h}$ before it was quenched with water $(20 \mathrm{~mL})$. The resulting mixture was extracted with EtOAc $(3 \times 50 \mathrm{~mL})$, and the combined organic layers were washed with water $(2 \times 20 \mathrm{~mL})$, brine $(20 \mathrm{~mL})$, dried $\left(\mathrm{Na}_{2} \mathrm{SO}_{4}\right)$ and concentrated in vacuo. Flash column chromatography (silica gel, hexanes:EtOAc:Et ${ }_{3} \mathrm{~N}$ 4:1:0.05) afforded tetraene 33 (426 mg, 67\%) 


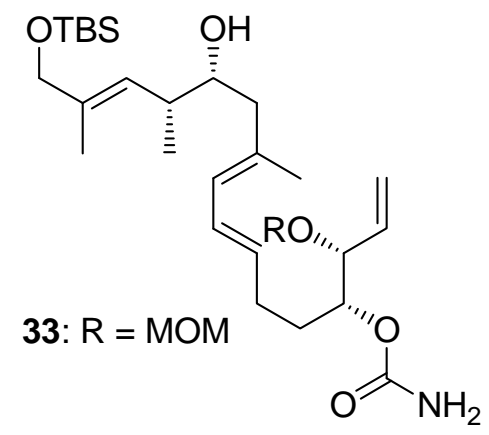

as a pale yellow viscous oil. 33: $R_{\mathrm{f}}=0.30$ (silica gel, hexanes:EtOAc 3:2); $[\alpha]_{\mathrm{D}}{ }^{25}=-3.0\left(\mathrm{CH}_{2} \mathrm{Cl}_{2}, c=0.25\right)$; IR (film) $V_{\max } 3460,2970,2929,1737,1441,1373,1230,1032 \mathrm{~cm}^{-1} ;{ }^{1} \mathrm{H}$ $\operatorname{NMR}\left(600 \mathrm{MHz}, \mathrm{CDCl}_{3}\right): \delta=6.27(\mathrm{dd}, J=15.6,10.8 \mathrm{~Hz}, 1 \mathrm{H})$, $5.87(\mathrm{~d}, J=10.8 \mathrm{~Hz}, 1 \mathrm{H}), 5.73(\mathrm{ddd}, J=17.4,10.8,7.8 \mathrm{~Hz}, 1 \mathrm{H})$, $5.60(\mathrm{dt}, J=15.0,7.5 \mathrm{~Hz}, 1 \mathrm{H}), 5.33(\mathrm{brd}, J=10.2 \mathrm{~Hz}, 1 \mathrm{H}), 5.32(\mathrm{brd}, J=17.4 \mathrm{~Hz}, 1 \mathrm{H}), 5.27$ (d, $J=10.2 \mathrm{~Hz}, 1 \mathrm{H}), 4.89(\mathrm{dt}, J=7.8,5.4 \mathrm{~Hz}, 1 \mathrm{H}), 4.78$ (brs, $2 \mathrm{H}), 4.71(\mathrm{~d}, J=6.6 \mathrm{~Hz}, 1 \mathrm{H}), 4.59$ $(\mathrm{d}, J=6.6 \mathrm{~Hz}, 1 \mathrm{H}), 4.12(\mathrm{dd}, J=7.8,4.2 \mathrm{~Hz}, 1 \mathrm{H}), 4.03(\mathrm{~s}, 2 \mathrm{H}), 3.50(\mathrm{ddd}, J=10.2,6.0,3.0 \mathrm{~Hz}$, $1 \mathrm{H}), 3.40$ (s, $3 \mathrm{H}), 2.47$ (ddq, $J=10.2,6.6,6.6 \mathrm{~Hz}, 1 \mathrm{H}), 2.31(\mathrm{~d}, J=13.8 \mathrm{~Hz}, 1 \mathrm{H}), 2.23-2.14$ (m, $2 \mathrm{H}), 1.95$ (dd, $J=13.2,9.6 \mathrm{~Hz}, 1 \mathrm{H}), 1.79-1.69(\mathrm{~m}, 2 \mathrm{H}), 1.75(\mathrm{~s}, 3 \mathrm{H}), 1.62(\mathrm{~s}, 3 \mathrm{H}), 1.04$ $(\mathrm{d}, J=6.6 \mathrm{~Hz}, 3 \mathrm{H}), 0.92(\mathrm{~s}, 9 \mathrm{H}), 0.07 \mathrm{ppm}(\mathrm{s}, 6 \mathrm{H}) ;{ }^{13} \mathrm{C} \mathrm{NMR}\left(150 \mathrm{MHz}, \mathrm{CDCl}_{3}\right): \delta=156.6$, 135.0, 134.0, 133.6, 132.0, 127.7, 126.9, 126.6, 119.5, 94.0, 77.8, 75.3, 73.0, 68.4, 55.7, 45.6, 38.3, 30.5, 28.7, 25.9, 18.4, 16.7, 16.5, 13.9, -5.2 ppm; HRMS (ESI): calcd for $\mathrm{C}_{28} \mathrm{H}_{51} \mathrm{NO}_{6} \mathrm{SiNa}^{+}$ $\left[\mathrm{M}+\mathrm{Na}^{+}\right] 548.3378$, found 548.3415 .

Ester 34: To a solution of acid $4(18 \mathrm{mg}, 8.36 \mu \mathrm{mol})$ in toluene $(3.0 \mathrm{~mL})$ were added $\mathrm{Et}_{3} \mathrm{~N}(21$ $\mu \mathrm{L}, 12.5 \mu \mathrm{mol})$ and 2,4,6-tichlorobenzoyl chloride $(16 \mu \mathrm{L}, 10.0 \mu \mathrm{mol})$ at room temperature. The reaction mixture was stirred for $1 \mathrm{~h}$ before a solution of alcohol $33(40 \mathrm{mg}, 7.6 \mu \mathrm{mol})$ and $4-$ DMAP $(9 \mathrm{mg}, 7.6 \mu \mathrm{mol})$ in toluene $(1.0 \mathrm{~mL})$ was added. The reaction mixture was stirred for 12 h before it was quenched with water $(5 \mathrm{~mL})$. The resulting mixture was extracted with EtOAc (2 $\times 15 \mathrm{~mL})$, and the combined organic layers were washed with brine $(10 \mathrm{~mL})$, dried $\left(\mathrm{Na}_{2} \mathrm{SO}_{4}\right)$ and concentrated in vacuo. Flash column chromatography (silica gel, hexanes:EtOAc:Et ${ }_{3} \mathrm{~N}$ 4:1:0.05) afforded ester 34 (33.5 mg, 61\%) as a colorless oil. 34: $R_{\mathrm{f}}=0.35$ (silica gel, hexanes:EtOAc 3:2); 


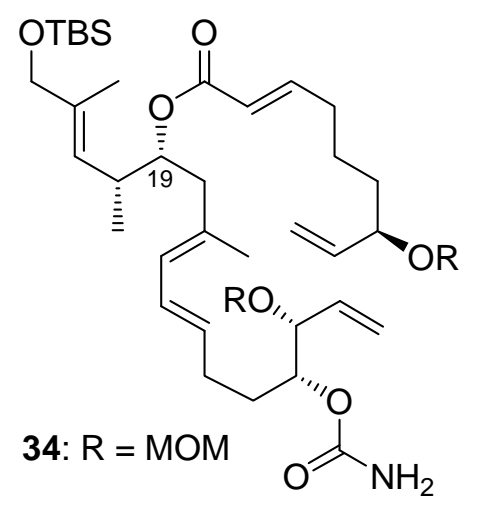

$[\alpha]_{\mathrm{D}}^{25}=+3.8\left(\mathrm{CH}_{2} \mathrm{Cl}_{2}, c=1.0\right)$; IR (film) $v_{\max } 3356,2930,2857$, $1719,1443,1385,1255,1098 \mathrm{~cm}^{-1} ;{ }^{1} \mathrm{H}$ NMR $\left(600 \mathrm{MHz}, \mathrm{CDCl}_{3}\right): \delta$ $=6.90(\mathrm{dt}, J=15.6,6.9 \mathrm{~Hz}, 1 \mathrm{H}), 6.20(\mathrm{dd}, J=15.0,10.8 \mathrm{~Hz}, 1 \mathrm{H})$, $5.78(\mathrm{~d}, J=15.6 \mathrm{~Hz}, 1 \mathrm{H}), 5.74(\mathrm{~d}, J=10.2 \mathrm{~Hz}, 1 \mathrm{H}), 5.72(\mathrm{ddd}, J=$ 17.4, 10.2, 7.2 Hz, $1 \mathrm{H}), 5.66$ (ddd, $J=17.4,10.8,7.8 \mathrm{~Hz}, 1 \mathrm{H})$, $5.52(\mathrm{dt}, J=15.0,7.2 \mathrm{~Hz}, 1 \mathrm{H}), 5.32(\mathrm{brd}, J=10.2 \mathrm{~Hz}, 1 \mathrm{H}), 5.31$ (brd, $J=17.4 \mathrm{~Hz}, 1 \mathrm{H}), 5.25$ (d, $J=10.2 \mathrm{~Hz}, 1 \mathrm{H}), 5.21$ (brd, $J=9.6 \mathrm{~Hz}, 1 \mathrm{H}), 5.20$ (brd, $J=$ $17.4 \mathrm{~Hz}, 1 \mathrm{H}), 4.95$ (ddd, $J=11.4,7.2,4.2 \mathrm{~Hz}, 1 \mathrm{H}), 4.88$ (dt, $J=8.4,4.8 \mathrm{~Hz}, 1 \mathrm{H}), 4.76$ (brs, 2 H), $4.71(\mathrm{~d}, J=6.6 \mathrm{~Hz}, 1 \mathrm{H}), 4.70(\mathrm{~d}, J=7.2 \mathrm{~Hz}, 1 \mathrm{H}), 4.58(\mathrm{~d}, J=7.2 \mathrm{~Hz}, 1 \mathrm{H}), 4.54(\mathrm{~d}, J=6.6$ Hz, $1 \mathrm{H}), 4.11$ (dd, $J=7.2,5.4 \mathrm{~Hz}, 1 \mathrm{H}), 4.03-3.96$ (m, $2 \mathrm{H}), 4.01$ (s, $2 \mathrm{H}), 3.39$ (s, $3 \mathrm{H}), 3.37$ (s, $3 \mathrm{H}), 2.65$ (ddq, $J=10.2,7.2,6.6 \mathrm{~Hz}, 1 \mathrm{H}), 2.31$ (dd, $J=13.8,3.6 \mathrm{~Hz}, 1 \mathrm{H}), 2.24-2.13$ (m, $4 \mathrm{H})$, 1.76-1.48 (m, 6 H), 1.72 (s, 3 H), 1.58 (s, 3 H), 0.96 (d, $J=7.2 \mathrm{~Hz}, 3 \mathrm{H}), 0.93$ (s, $9 \mathrm{H}), 0.06 \mathrm{ppm}$ $(\mathrm{s}, 6 \mathrm{H}) ;{ }^{13} \mathrm{C} \mathrm{NMR}\left(150 \mathrm{MHz}, \mathrm{CDCl}_{3}\right): \delta=166.3,156.5,148.6,138.1,135.3,134.0,132.7,131.3$, 127.5, 127.1, 125.6, 121.7, 119.4, 117.4, 94.0, 93.7, 77.8, 77.0, 75.8, 75.4, 68.2, 55.7, 55.4, 42.4, $35.9,34.8,32.0,30.5,28.7,25.9,23.8,18.4,16.8,16.6,13.8,-5.2,-5.2$ ppm; HRMS (ESI): calcd for $\mathrm{C}_{39} \mathrm{H}_{67} \mathrm{NO}_{9} \mathrm{SiNa}^{+}\left[\mathrm{M}+\mathrm{Na}^{+}\right]$744.4477, found 744.4498 .

Ester 35: To a solution of acid $4(334 \mathrm{mg}, 1.56 \mathrm{mmol})$ in toluene $(15 \mathrm{~mL})$ were added $\mathrm{Et}_{3} \mathrm{~N}$ $(0.40 \mathrm{~mL}, 2.90 \mathrm{mmol})$ and 2,4,6-tichlorobenzoyl chloride $(0.28 \mathrm{~mL}, 1.81 \mathrm{mmol})$ at room temperature followed by a solution of alcohol $2(600 \mathrm{mg}, 1.42 \mathrm{mmol})$ and 4-DMAP (173 $\mathrm{mg}$, $1.41 \mathrm{mmol})$ in toluene $(10 \mathrm{~mL})$. The reaction mixture was stirred for $12 \mathrm{~h}$ before it was diluted with $\mathrm{H}_{2} \mathrm{O}(20 \mathrm{~mL})$ and EtOAc $(50 \mathrm{~mL})$. The resulting mixture was extracted with EtOAc $(2 \times 50$ $\mathrm{mL})$, and the combined organic layers were washed with brine $(20 \mathrm{~mL})$, dried $\left(\mathrm{Na}_{2} \mathrm{SO}_{4}\right)$ and 
concentrated in vacuo. Flash column chromatography (silica gel,

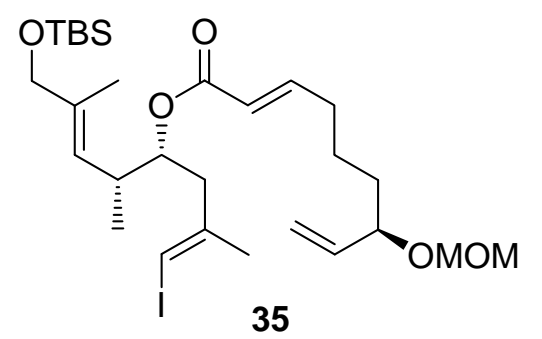
hexanes:EtOAc:Et $t_{3} \mathrm{~N}$ 4:1:0.05) afforded ester 35 (774 mg, 88\%) as a colorless oil. 35: $R_{\mathrm{f}}=0.24$ (silica gel, hexanes:EtOAc 95:5); $[\alpha]_{\mathrm{D}}^{25}=+34.5\left(\mathrm{CHCl}_{3}, c=2.0\right)$; IR (film) $v_{\max } 2929,1720,1654$, $1462,1256,1033,837 \mathrm{~cm}^{-1} ;{ }^{1} \mathrm{H}$ NMR $\left(600 \mathrm{MHz}, \mathrm{CDCl}_{3}\right): \delta=$ $6.94(\mathrm{ddd}, J=15.6,7.0,7.0 \mathrm{~Hz}, 1 \mathrm{H}), 5.89(\mathrm{~d}, J=0.5 \mathrm{~Hz}, 1 \mathrm{H}), 5.79$ (ddd, $J=15.6,1.5,1.5 \mathrm{~Hz}$ $1 \mathrm{H}), 5.68$ (ddd, $J=17.4,10.3,7.6 \mathrm{~Hz}, 1 \mathrm{H}), 5.25-5.19$ (m, $3 \mathrm{H}$ ), 4.94 (ddd, $J=11.2,7.7,3.5 \mathrm{~Hz}$, $1 \mathrm{H}), 4.71(\mathrm{~d}, J=6.8 \mathrm{~Hz}, 1 \mathrm{H}), 4.54(\mathrm{~d}, J=6.8 \mathrm{~Hz}, 1 \mathrm{H}), 4.54(\mathrm{brs}, 3 \mathrm{H}), 3.38(\mathrm{~s}, 3 \mathrm{H}), 2.65(\mathrm{~m}, 1$ H), $2.46(\mathrm{~m}, 1 \mathrm{H}), 2.39$ (dd, $J=13.8,9.0 \mathrm{~Hz}, 1 \mathrm{H}), 2.26(\mathrm{~m}, 2 \mathrm{H}), 1.84(\mathrm{~s}, 3 \mathrm{H}), 1.59$ (d, $J=1.3$ $\mathrm{Hz}, 1 \mathrm{H}), 1.66-1.50(\mathrm{~m}, 6 \mathrm{H}), 0.96(\mathrm{~d}, J=6.7 \mathrm{~Hz}, 3 \mathrm{H}), 0.92(\mathrm{~s}, 9 \mathrm{H}), 0.07 \mathrm{ppm}(\mathrm{s}, 6 \mathrm{H}) ;{ }^{13} \mathrm{C}$ $\operatorname{NMR}\left(150 \mathrm{MHz}, \mathrm{CDCl}_{3}\right): \delta=166.1,149.1,144.3,138.2,135.8,125.0,121.4,117.4,93.8,77.2$ $77.0,74.8,68.1,55.5,42.1,36.1,34.8,32.1,25.9 \times 3,24.0,23.8,18.4,16.8,13.9,-5.2 \times 2$; HRMS (ESI): calcd for $\mathrm{C}_{28} \mathrm{H}_{49} \mathrm{IO}_{5} \mathrm{SiNa}^{+}\left[\mathrm{M}+\mathrm{Na}^{+}\right]$643.2286, found 643.2276 .

Hexaene 34: To a mixture of stannane 3 (790 mg, $1.52 \mathrm{mmol}$ ) and vinyl iodide 35 (730 mg, 1.17 mmol) in NMP (15 mL, degassed by using the freeze-thaw process sequence three times) were

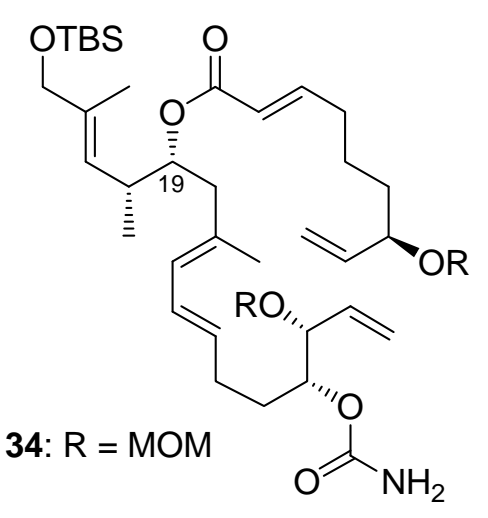
added $\mathrm{Pd}(\mathrm{dba})_{2}(135 \mathrm{mg}, 0.23 \mathrm{mmol}), \mathrm{AsPh}_{3}(1.08 \mathrm{~g}, 3.52 \mathrm{mmol})$ and $\mathrm{LiCl}(99 \mathrm{mg}, 2.35 \mathrm{mmol}$, freshly dried by heating with a heatgun for 20 min under vacuum) at room temperature. The reaction mixture was stirred for $12 \mathrm{~h}$ before it was quenched with water $(10 \mathrm{~mL})$. The resulting mixture was extracted with EtOAc $(3 \times 50 \mathrm{~mL})$, and the combined organic layers were washed with water $(2 \times 20 \mathrm{~mL})$, brine $(20 \mathrm{~mL})$, dried $\left(\mathrm{Na}_{2} \mathrm{SO}_{4}\right)$ and concentrated in vacuo. Flash column 
chromatography (silica gel, hexanes:EtOAc: $\mathrm{Et}_{3} \mathrm{~N}$ 3:2:0.01) afforded hexaene 34 (567 mg, 67\%) as a colorless oil. 34: $R_{\mathrm{f}}=0.35$ (silica gel, hexanes:EtOAc 3:2); $[\alpha]_{\mathrm{D}}{ }^{25}=+3.8\left(\mathrm{CH}_{2} \mathrm{Cl}_{2}, c=1.0\right)$; IR (film) $v_{\max } 3356,2930,2857,1719,1443,1385,1255,1098 \mathrm{~cm}^{-1} ;{ }^{1} \mathrm{H}$ NMR $(600 \mathrm{MHz}$, $\left.\mathrm{CDCl}_{3}\right): \delta=6.90(\mathrm{dt}, J=15.6,6.9 \mathrm{~Hz}, 1 \mathrm{H}), 6.20(\mathrm{dd}, J=15.0,10.8 \mathrm{~Hz}, 1 \mathrm{H}), 5.78(\mathrm{~d}, J=15.6$ $\mathrm{Hz}, 1 \mathrm{H}), 5.74(\mathrm{~d}, J=10.2 \mathrm{~Hz}, 1 \mathrm{H}), 5.72$ (ddd, $J=17.4,10.2,7.2 \mathrm{~Hz}, 1 \mathrm{H}), 5.66$ (ddd, $J=17.4$, 10.8, $7.8 \mathrm{~Hz}, 1 \mathrm{H}), 5.52$ (dt, $J=15.0,7.2 \mathrm{~Hz}, 1 \mathrm{H}), 5.32$ (brd, $J=10.2 \mathrm{~Hz}, 1 \mathrm{H}), 5.31$ (brd, $J=$ $17.4 \mathrm{~Hz}, 1 \mathrm{H}), 5.25$ (d, $J=10.2 \mathrm{~Hz}, 1 \mathrm{H}), 5.21(\mathrm{brd}, J=9.6 \mathrm{~Hz}, 1 \mathrm{H}), 5.20($ brd, $J=17.4 \mathrm{~Hz}, 1$ H), 4.95 (ddd, $J=11.4,7.2,4.2 \mathrm{~Hz}, 1 \mathrm{H}), 4.88$ (dt, $J=8.4,4.8 \mathrm{~Hz}, 1 \mathrm{H}), 4.76$ (brs, $2 \mathrm{H}), 4.71$ (d, $J=6.6 \mathrm{~Hz}, 1 \mathrm{H}), 4.70(\mathrm{~d}, J=7.2 \mathrm{~Hz}, 1 \mathrm{H}), 4.58(\mathrm{~d}, J=7.2 \mathrm{~Hz}, 1 \mathrm{H}), 4.54(\mathrm{~d}, J=6.6 \mathrm{~Hz}, 1 \mathrm{H})$ $4.11(\mathrm{dd}, J=7.2,5.4 \mathrm{~Hz}, 1 \mathrm{H}), 4.03-3.96(\mathrm{~m}, 2 \mathrm{H}), 4.01(\mathrm{~s}, 2 \mathrm{H}), 3.39(\mathrm{~s}, 3 \mathrm{H}), 3.37$ (s, $3 \mathrm{H})$, $2.65(\mathrm{ddq}, J=10.2,7.2,6.6 \mathrm{~Hz}, 1 \mathrm{H}), 2.31(\mathrm{dd}, J=13.8,3.6 \mathrm{~Hz}, 1 \mathrm{H}), 2.24-2.13(\mathrm{~m}, 4 \mathrm{H})$, 1.76-1.48 (m, $6 \mathrm{H}), 1.72$ (s, $3 \mathrm{H}), 1.58$ (s, $3 \mathrm{H}), 0.96$ (d, $J=7.2 \mathrm{~Hz}, 3 \mathrm{H}), 0.93(\mathrm{~s}, 9 \mathrm{H}), 0.06 \mathrm{ppm}$ $(\mathrm{s}, 6 \mathrm{H}) ;{ }^{13} \mathrm{C} \mathrm{NMR}\left(150 \mathrm{MHz}, \mathrm{CDCl}_{3}\right): \delta=166.3,156.5,148.6,138.1,135.3,134.0,132.7,131.3$, 127.5, 127.1, 125.6, 121.7, 119.4, 117.4, 94.0, 93.7, 77.8, 77.0, 75.8, 75.4, 68.2, 55.7, 55.4, 42.4, $35.9,34.8,32.0,30.5,28.7,25.9,23.8,18.4,16.8,16.6,13.8,-5.2,-5.2$ ppm; HRMS (ESI): calcd for $\mathrm{C}_{39} \mathrm{H}_{67} \mathrm{NO}_{9} \mathrm{SiNa}^{+}\left[\mathrm{M}+\mathrm{Na}^{+}\right]$744.4477, found 744.4498 .

Alcohol 36: To a solution of TBS ether $34(500 \mathrm{mg}, 0.69 \mathrm{mmol})$ in THF $(10 \mathrm{~mL})$ was added TBAF (1.0 M in THF, $1.03 \mathrm{~mL}, 1.03 \mathrm{mmol})$ at room temperature. The reaction mixture was stirred for $2 \mathrm{~h}$ before it was concentrated in vacuo. The crude residue was diluted with water (5 $\mathrm{mL})$, extracted with EtOAc $(3 \times 20 \mathrm{~mL})$, and the combined organic layers were washed with brine $(5 \mathrm{~mL})$, dried $\left(\mathrm{Na}_{2} \mathrm{SO}_{4}\right)$ and concentrated in vacuo. Flash column chromatorgraphy (silica gel, hexanes:EtOAc:Et $3 \mathrm{~N}$ 7:3:1) afforded alcohol $36(328 \mathrm{mg}, 78 \%)$ as a viscous oil. 36: $R_{\mathrm{f}}=$ 


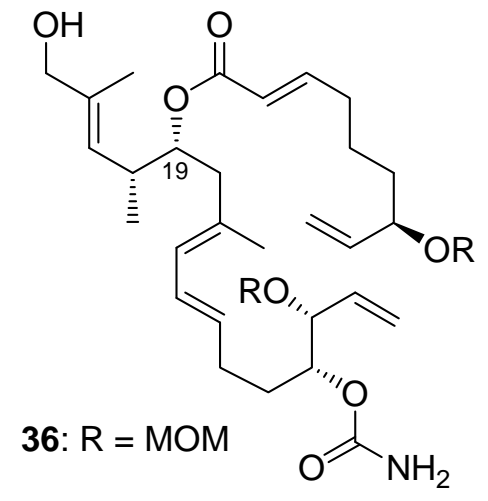

0.36 (silica gel, hexanes:EtOAc 3:2); $[\alpha]_{\mathrm{D}}^{25}=+5.1(\mathrm{MeOH}, c=$ 2.0); IR (film) $v_{\max } 3450,2931,1716,1386,1319,1268,1030$ $\mathrm{cm}^{-1} ;{ }^{1} \mathrm{H}$ NMR $\left(600 \mathrm{MHz}, \mathrm{CDCl}_{3}\right): \delta=6.92(\mathrm{dt}, J=13.9,6.9 \mathrm{~Hz}$, $1 \mathrm{H}), 6.20(\mathrm{dd}, J=15.0,10.8 \mathrm{~Hz}, 1 \mathrm{H}), 5.80(\mathrm{~d}, J=15.7 \mathrm{~Hz}, 1 \mathrm{H})$, $5.76(\mathrm{~d}, J=10.6 \mathrm{~Hz}, 1 \mathrm{H}),(\mathrm{ddd}, J=17.5,10.4,7.4 \mathrm{~Hz}, 1 \mathrm{H}), 5.67$ (ddd, $J=17.6,10.4,7.7 \mathrm{~Hz}, 1 \mathrm{H}), 5.55$ (ddd. $J=14.4,7.0,7.0 \mathrm{~Hz}$, $1 \mathrm{H}), 5.33(\mathrm{~m}, 2 \mathrm{H}), 5.27(\mathrm{dd}, J=9.9,1.2 \mathrm{~Hz}, 1 \mathrm{H}), 5.22(\mathrm{~m}, 2 \mathrm{H})$, 4.98 (ddd, $J=11.2,6.8,4.5 \mathrm{~Hz}, 1 \mathrm{H}), 4.89$ (ddd. $J=9.8,4.9,4.9 \mathrm{~Hz}, 1 \mathrm{H}), 4.73-4.65$ (brs, $2 \mathrm{H}$ ), $4.72(\mathrm{~d}, J=2.4 \mathrm{~Hz}, 1 \mathrm{H}), 4.71(\mathrm{~d}, J=2.4 \mathrm{~Hz}, 1 \mathrm{H}), 4.59(\mathrm{~d}, J=7.2 \mathrm{~Hz}, 1 \mathrm{H}), 4.54(\mathrm{~d}, J=7.2 \mathrm{~Hz}$, $1 \mathrm{H}), 4.12(\mathrm{~m}, 1 \mathrm{H}), 4.02(\mathrm{brs}, 3 \mathrm{H}), 3.40(\mathrm{~s}, 3 \mathrm{H}), 3.38(\mathrm{~s}, 3 \mathrm{H}), 2.67$ (m, $1 \mathrm{H}), 2.30$ (dd, $J=13.8$, $3.9 \mathrm{~Hz}, 1 \mathrm{H}), 2.26-2.21(\mathrm{~m}, 3 \mathrm{H}), 2.21-2.14(\mathrm{~m}, 2 \mathrm{H}), 1.74(\mathrm{~s}, 3 \mathrm{H}), 1.67$ (d, $J=1.1 \mathrm{~Hz}, 3 \mathrm{H})$, $1.78-1.69(\mathrm{~m}, 1 \mathrm{H}), 1.68-1.48(\mathrm{~m}, 6 \mathrm{H}), 0.98 \mathrm{ppm}(\mathrm{d}, J=6.7 \mathrm{~Hz}, 3 \mathrm{H}) ;{ }^{13} \mathrm{C}$ NMR $(150 \mathrm{MHz}$ $\left.\mathrm{CDCl}_{3}\right): \delta=166.3,156.7,148.5,138.2,135.6,134.0,132.5,131.5,127.6,127.3,127.1,121.6$ 119.4, 117.4, 94.0, 93.7, 77.8, 76.9, 75.5, 75.3, 68.6, 55.7, 55.4, 42.5, 36.0, 34.8, 32.0, 30.4, 28.6, 23.8, 16.8, 16.4, 14.0; HRMS (ESI): calcd for $\mathrm{C}_{33} \mathrm{H}_{53} \mathrm{NO}_{9} \mathrm{Na}^{+}\left[\mathrm{M}+\mathrm{Na}^{+}\right] 630.3613$, found 630.3620.

Aldehyde 37: To a solution of alcohol 36 (282 mg, $0.464 \mathrm{mmol})$ in $\mathrm{CH}_{2} \mathrm{Cl}_{2}(3.0 \mathrm{~mL})$ were added $\mathrm{NaHCO}_{3}(242 \mathrm{mg}, 2.88 \mathrm{mmol})$ and DMP $(197 \mathrm{mg}, 0.557 \mathrm{mmol})$ at room temperature. The reaction mixture was stirred for 30 min before it was quenched with $\mathrm{NaHCO}_{3}(5 \mathrm{~mL}$, sat. aq.) and $\mathrm{Na}_{2} \mathrm{~S}_{2} \mathrm{O}_{3}(5 \mathrm{~mL}$, sat. aq.). The resulting mixture was extracted with EtOAc $(2 \times 50 \mathrm{~mL})$, and the combined organic layers were washed with brine $(10 \mathrm{~mL})$, dried $\left(\mathrm{Na}_{2} \mathrm{SO}_{4}\right)$, and concentrated in vacuo. Flash chromatography (silica gel, hexanes:EtOAc:Et ${ }_{3} \mathrm{~N}$ 1:1:0.01) afforded aldehyde 37 


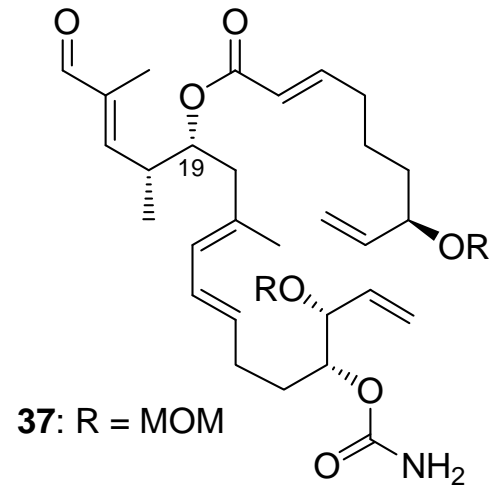

(238 mg, 84\%) as a colorless oil. 37: $R_{\mathrm{f}}=0.35$ (silica gel, hexanes:EtOAc 2:3); $[\alpha]_{\mathrm{D}}^{25}=+9.4 \quad\left(\mathrm{CHCl}_{3}, c=1.0\right)$; IR (film) $V_{\max } 3450,2931,1716,1386,1319,1268,1030 \mathrm{~cm}^{-1} ;{ }^{1} \mathrm{H}$ NMR $\left(600 \mathrm{MHz}, \mathrm{CDCl}_{3}\right): \delta=9.42(\mathrm{~s}, 1 \mathrm{H}), 6.95(\mathrm{ddd}, J=13.9,6.9,6.9$ $\mathrm{Hz}, 1 \mathrm{H}), 6.33(\mathrm{~d}, J=10.1 \mathrm{~Hz}, 1 \mathrm{H}), 6.20(\mathrm{dd}, J=15.1,10.8 \mathrm{~Hz}, 1$ H), $5.80(\mathrm{~d}, J=15.6 \mathrm{~Hz}, 1 \mathrm{H}), 5.76(\mathrm{~d}, J=10.9 \mathrm{~Hz}, 1 \mathrm{H}), 5.74(\mathrm{~m}$, $1 \mathrm{H}), 5.67$ (ddd, $J=17.0,10.5,7.6 \mathrm{~Hz}, 1 \mathrm{H}), 5.56,(\mathrm{~m}, 1 \mathrm{H}), 5.36-5.30(\mathrm{~m}, 2 \mathrm{H}), 5.25-5.19(\mathrm{~m}, 1$ H), $5.10(\mathrm{~m}, 1 \mathrm{H}), 4.89(\mathrm{bm}, 1 \mathrm{H}), 4.72(\mathrm{~d}, J=2.6 \mathrm{~Hz}, 1 \mathrm{H}), 4.71(\mathrm{~d}, J=2.6 \mathrm{~Hz}, 1 \mathrm{H}), 4.74-4.60$ (brs, $2 \mathrm{H}), 4.71(\mathrm{~d}, J=6.8 \mathrm{~Hz}, 1 \mathrm{H}), 4.71(\mathrm{~d}, J=6.8 \mathrm{~Hz}, 1 \mathrm{H}), 4.12(\mathrm{~m}, 1 \mathrm{H}), 4.01(\mathrm{~m}, 1 \mathrm{H}), 3.40$ (s, 3 H), 3.39 (s, 3 H), 2.98 (m, 1 H), 2.30-2.10 (m, 6 H), 1.73 (s, 3 H), 1.61 (s, 3 H), 1.80-1.69 (m, $5 \mathrm{H}), 1.68-1.45(\mathrm{~m}, 2 \mathrm{H}), 1.11 \mathrm{ppm}(\mathrm{d}, J=6.8 \mathrm{~Hz}, 3 \mathrm{H}) ;{ }^{13} \mathrm{C}$ NMR $\left(150 \mathrm{MHz}, \mathrm{CDCl}_{3}\right): \delta=$ 166.3, 156.7, 148.4, 138.1, 135.6, 134.0, 132.5, 131.5, 127.6, 127.3, 127.1, 121.6, 119.4, 117.4, $94.0,93.7,77.8,76.9,75.5,75.3,68.6,55.7,55.4,42.5,36.0,34.8,32.0,30.4,28.6,23.8,16.8$, 16.4, 14.0; HRMS (ESI): calcd for $\mathrm{C}_{33} \mathrm{H}_{51} \mathrm{NO}_{9} \mathrm{Na}^{+}\left[\mathrm{M}+\mathrm{Na}^{+}\right]$628.3456, found 628.3447.

Vinyl iodide 38: To a solution of $\mathrm{CrCl}_{2}$ (426 mg, $3.46 \mathrm{mmol}$, flame-dried) in $\mathrm{THF}$ ( $3 \mathrm{~mL}$ ) was added a solution of aldehyde $37(215 \mathrm{mg}, 0.35 \mathrm{mmol})$ and $\mathrm{CHI}_{3}(410 \mathrm{mg}, 1.04 \mathrm{mmol})$ in dioxane $(18 \mathrm{~mL})$ at room temperature. The reaction mixture was stirred for $2 \mathrm{~h}$ before it was quenched with $\mathrm{NaHCO}_{3}(20 \mathrm{~mL}$, sat. aq.). The resulting mixture was diluted with EtOAc $(50 \mathrm{~mL})$ and filtered through a short pad of Celite ${ }^{\circledR}$. The aqueous layer was separated and extracted with EtOAc $(3 \times 20 \mathrm{~mL})$, and the combined organic layers were washed with brine $(10 \mathrm{~mL})$, dried $\left(\mathrm{Na}_{2} \mathrm{SO}_{4}\right)$ and concentrated in vacuo. Flash column chromatography (silica gel, hexanes:EtOAc: $\mathrm{Et}_{3} \mathrm{~N}$ 6.5:3.5:0.05) afforded vinyl iodide $38(205 \mathrm{mg}, 80 \%)$ as a white gummy 


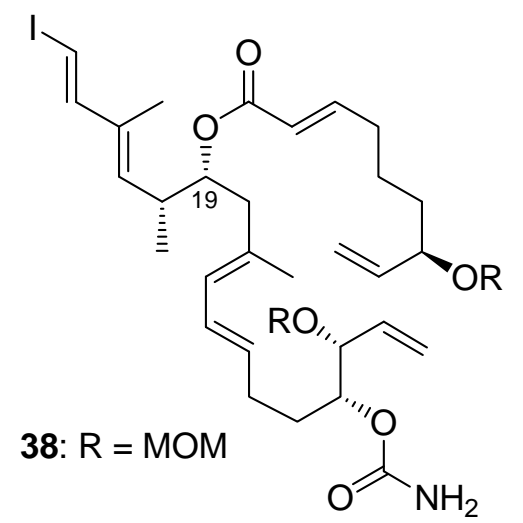

solid. 38: $R_{\mathrm{f}}=0.48$ (silica gel, hexanes:EtOAc 3:2); $[\alpha]_{\mathrm{D}}^{25}=$ $+13.5\left(\mathrm{CHCl}_{3}, c=0.48\right)$; IR (film) $v_{\max } 3467,2928,1717,1381$, 1149, 1096, 1030, $919 \mathrm{~cm}^{-1} ;{ }^{1} \mathrm{H}$ NMR $\left(600 \mathrm{MHz}, \mathrm{CDCl}_{3}\right): \delta=$ $7.05(\mathrm{~d}, J=14.6 \mathrm{~Hz}, 1 \mathrm{H}), 6.92(\mathrm{ddd}, J=15.7,6.9,1.7 \mathrm{~Hz}, 1 \mathrm{H})$, $6.21(\mathrm{~d}, J=14.6 \mathrm{~Hz}, 1 \mathrm{H}), 6.20(\mathrm{dd}, J=14.9,10.9 \mathrm{~Hz}, 1 \mathrm{H}), 5.79$ (d, $J=15.6 \mathrm{~Hz}, 1 \mathrm{H}), 5.74(\mathrm{~d}, J=11.0 \mathrm{~Hz}, 1 \mathrm{H}), 5.72(\mathrm{ddd}, J=$ 17.3, 10.3, $7.2 \mathrm{~Hz}, 1 \mathrm{H}), 5.68$ (ddd, $J=17.5,10.5,7.4 \mathrm{~Hz}, 1 \mathrm{H}), 5.54$ (d, $J=14.5,7.5,6.9 \mathrm{~Hz}, 1$ H), 5.30-5.35 (m, $3 \mathrm{H}), 5.20-5.25(\mathrm{~m}, 2 \mathrm{H}), 4.97$ (ddd, $J=8.6,6.8,4.1 \mathrm{~Hz}, 1 \mathrm{H}), 4.89$ (ddd, $J=$ 8.3, 4.9, $4.9 \mathrm{~Hz}, 1 \mathrm{H}), 4.72,(\mathrm{~d}, J=2.7 \mathrm{~Hz}, 1 \mathrm{H}), 4.70(\mathrm{~d}, J=2.7 \mathrm{~Hz}, 1 \mathrm{H}), 4.68-4.61$ (brs, $2 \mathrm{H})$, $4.59(\mathrm{~d}, J=6.8 \mathrm{~Hz}, 1 \mathrm{H}), 4.55(\mathrm{~d}, J=6.8 \mathrm{~Hz}, 1 \mathrm{H}), 4.12(\mathrm{dd}, J=7.3,2.4 \mathrm{~Hz}, 1 \mathrm{H}), 4.0(\mathrm{dd}, J=$ 12.8, $5.5 \mathrm{~Hz}, 1 \mathrm{H}), 3.40(\mathrm{~s}, 3 \mathrm{H}), 3.39(\mathrm{~s}, 3 \mathrm{H}), 2.74(\mathrm{dqd}, J=9.9,6.8,3.6 \mathrm{~Hz}, 1 \mathrm{H}), 2.30-2.15(\mathrm{~m}$, $6 \mathrm{H}), 1.76-1.70$ (m, $2 \mathrm{H}), 1.66-1.50(\mathrm{~m}, 4 \mathrm{H}), 1.73(\mathrm{~s}, 3 \mathrm{H}), 1.72$ (d, $J=1.1 \mathrm{~Hz}, 3 \mathrm{H}), 0.99 \mathrm{ppm}$ $(\mathrm{d}, J=6.8 \mathrm{~Hz}, 3 \mathrm{H}) ;{ }^{13} \mathrm{C} \mathrm{NMR}\left(150 \mathrm{MHz}, \mathrm{CDCl}_{3}\right): \delta=166.2,156.4,149.5,149.0,138.1,135.4$, $134.8,134.0,132.2,131.6,127.8,127.1,121.4,119.4,117.4,93.9,93.7,77.8,77.6,75.4,75.2$ $74.2,55.7,55.5,42.4,36.4,34.9,32.0,30.4,28.7,23.8,16.8,16.3,12.3$ ppm; HRMS (ESI): calcd for $\mathrm{C}_{34} \mathrm{H}_{52} \mathrm{INO}_{8} \mathrm{Na}^{+}\left[\mathrm{M}+\mathrm{Na}^{+}\right]$752.2630, found 752.2645.

Diol 39 and cyclic carbonate 40: To a solution bis-MOM ether 38 (145 mg, $0.201 \mathrm{mmol})$ in $\mathrm{MeOH}(15 \mathrm{~mL})$ was added TMSCl $(207 \mu \mathrm{L}, 1.206 \mathrm{mmol})$ at $40{ }^{\circ} \mathrm{C}$. The reaction mixture was stirred for $1 \mathrm{~h}$ before it was cooled to room temperature, quenched with $\mathrm{NaHCO}_{3}(300 \mathrm{mg})$ and concentrated in vacuo. The resulting residue was dissolved in EtOAc $(15 \mathrm{~mL})$, filtered through a pad of Celite ${ }^{\circledR}$, washed with EtOAc $(2 \times 10 \mathrm{~mL})$ and concentrated in vacuo. Flash column chromatography (silica gel, hexanes:EtOAc:Et $3 \mathrm{~N}$ 4:6:0.025 $\rightarrow$ 0:10:0.05) afforded diol 39 (87 


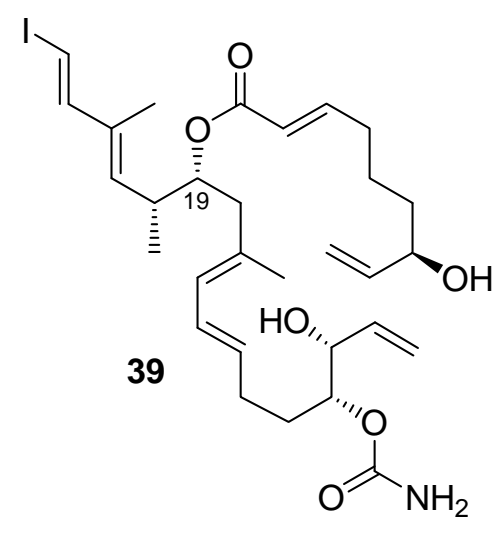

$\mathrm{mg}, 67 \%)$ as colorless gummy solid, and cyclic carbonate $\mathbf{4 0}(12$ mg, $10 \%$ ) as a gummy solid. 39: $R_{\mathrm{f}}=0.40$ (silica gel, hexanes:EtOAc 2:3); $[\alpha]_{\mathrm{D}}{ }^{25}=+52.8\left(\mathrm{CHCl}_{3}, c=1.35\right)$; IR (film) $v_{\max } 3364,2932,1706,1389,1317,1058,990, \mathrm{~cm}^{-1} ;{ }^{1} \mathrm{H}$ NMR $\left(600 \mathrm{MHz}, \mathrm{CDCl}_{3}\right): \delta=7.06(\mathrm{~d}, J=14.6 \mathrm{~Hz}, 1 \mathrm{H}), 6.90(\mathrm{dt}, J=$ 15.7, $6.9 \mathrm{~Hz}, 1 \mathrm{H}), 6.22(\mathrm{~d}, J=14.6 \mathrm{~Hz}, 1 \mathrm{H}), 6.20(\mathrm{dd}, J=15.0$,

$10.8 \mathrm{~Hz}, 1 \mathrm{H}), 5.92-5.85(\mathrm{~m}, 2 \mathrm{H}), 5.79(\mathrm{~d}, J=15.7 \mathrm{~Hz}, 1 \mathrm{H}), 5.74(\mathrm{~d}, J=10.8 \mathrm{~Hz}, 1 \mathrm{H}), 5.53(\mathrm{dt}$, $J=14.6,7.0 \mathrm{~Hz}, 1 \mathrm{H}), 5.37(\mathrm{~d}, J=17.3 \mathrm{~Hz}, 1 \mathrm{H}), 5.32(\mathrm{~d}, J=9.9 \mathrm{~Hz}, 1 \mathrm{H}), 5.26(\mathrm{~d}, J=1.2 \mathrm{~Hz}, 1$ H), $5.24(\mathrm{~d}, J=4.8 \mathrm{~Hz}, 1 \mathrm{H}), 4.97(\mathrm{ddd}, J=10.5,7.0,3.8 \mathrm{~Hz}, 1 \mathrm{H}), 4.75(\mathrm{bm}, 1 \mathrm{H}), 4.77-4.67$ (brs, $2 \mathrm{H}), 4.17-4.10(\mathrm{bm}, 2 \mathrm{H}), 2.73$ (ddq, $J=10.8,10.5,6.7 \mathrm{~Hz}, 1 \mathrm{H}), 2.28-2.12(\mathrm{~m}, 6 \mathrm{H})$, 1.97-1.78 (brs, $2 \mathrm{H}), 1.78-1.52$ (m, $2 \mathrm{H}), 1.73$ (s, $3 \mathrm{H}), 1.72$ (d, $J=0.9 \mathrm{~Hz}, 3 \mathrm{H}), 1.52-1.50$ (m, $5 \mathrm{H}), 1.00 \mathrm{ppm}(\mathrm{d}, J=6.7 \mathrm{~Hz}, 3 \mathrm{H}) ;{ }^{13} \mathrm{C} \mathrm{NMR}\left(150 \mathrm{MHz}, \mathrm{CDCl}_{3}\right): \delta=166.2,157.1,149.5,149.0$, $141.1,136.9,135.3,134.9,132.3,131.5,127.8,127.2,121.5,117.0,114.8,79.1,74.3,74.1,72.8$, 42.6, 36.6, 36.3, 32.0, 30.4, 28.7, 23.9, 16.8, 16.7, 16.4, 12.3 ppm; HRMS (ESI): calcd for $\mathrm{C}_{30} \mathrm{H}_{44} \mathrm{INO}_{6} \mathrm{Na}^{+}\left[\mathrm{M}+\mathrm{Na}^{+}\right]$664.2106, found 664.2119.

Cyclic carbonate 40: $R_{\mathrm{f}}=0.30$ (silica gel, hexanes:EtOAc 7:3);

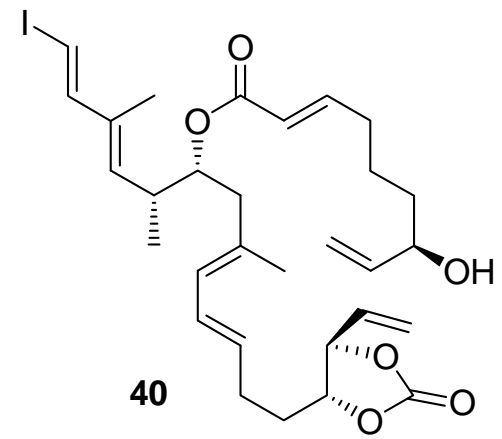

$[\alpha]_{\mathrm{D}}^{25}=+48.3\left(\mathrm{CHCl}_{3}, c=0.86\right) ;$ IR (film) $v_{\max } 3491,2926,1804$, $1713,1268,1186,1048,988 \mathrm{~cm}^{-1} ;{ }^{1} \mathrm{H}$ NMR $\left(600 \mathrm{MHz}, \mathrm{CDCl}_{3}\right)$ : $\delta=7.06(\mathrm{~d}, J=14.7 \mathrm{~Hz}, 1 \mathrm{H}), 6.93(\mathrm{dt}, J=15.2,6.7 \mathrm{~Hz}, 1 \mathrm{H})$, $6.22(\mathrm{~d}, J=14.7 \mathrm{~Hz}, 1 \mathrm{H}), 6.23(\mathrm{dd}, J=17.2,10.4 \mathrm{~Hz}, 1 \mathrm{H}), 5.86$ (m, $2 \mathrm{H}), 5.79(\mathrm{dd}, J=15.7,0.8 \mathrm{~Hz}, 1 \mathrm{H}), 5.74(\mathrm{~d}, J=10.8 \mathrm{~Hz}, 1$ H), $5.53(\mathrm{~m}, 2 \mathrm{H}), 5.44(\mathrm{~d}, J=10.6 \mathrm{~Hz}, 1 \mathrm{H}), 5.31(\mathrm{~d}, J=10.0 \mathrm{~Hz}, 1 \mathrm{H}), 5.24(\mathrm{dt}, J=17.2,1.0$ $\mathrm{Hz}, 1 \mathrm{H}), 5.14(\mathrm{dt}, J=10.4,0.9 \mathrm{~Hz}, 1 \mathrm{H}), 4.98(\mathrm{ddd}, J=11.0,7.0,4.0 \mathrm{~Hz}, 1 \mathrm{H}), 4.66$ (t, $J=7.3$ 
$\mathrm{Hz}, 1 \mathrm{H}), 4.33$ (ddd, $J=7.6,7.6,4.7 \mathrm{~Hz}, 1 \mathrm{H}), 4.13$ (bm, $1 \mathrm{H}), 4.12$ (ddq, $J=11.0,10.8,6.6 \mathrm{~Hz}$

$1 \mathrm{H}), 2.38-2.18$ (m, $6 \mathrm{H}), 1.95-1.70(\mathrm{~m}, 4 \mathrm{H}), 1.74$ (s, $3 \mathrm{H}), 1.72(\mathrm{~s}, 3 \mathrm{H}), 1.70-1.50$ (m, $6 \mathrm{H})$, $1.00 \mathrm{ppm}(\mathrm{d}, J=6.6 \mathrm{~Hz}, 3 \mathrm{H}) ;{ }^{13} \mathrm{C} \mathrm{NMR}\left(150 \mathrm{MHz}, \mathrm{CDCl}_{3}\right): \delta=166.1,154.2149 .5,149.0$, 141.0, 135.3, 134.9, 133.5, 132.0, 129.5, 128.3, 127.2, 121.4, 121.4, 114.9, 82.6, 81.1, 75.1, 74.3, $72.9,42.5,36.5,36.3,32.8,32.1,28.0,23.8,16.7,16.4,12.3$ ppm; HRMS (ESI): calcd for $\mathrm{C}_{30} \mathrm{H}_{41} \mathrm{IO}_{6} \mathrm{Na}^{+}\left[\mathrm{M}+\mathrm{Na}^{+}\right]$647.1840, found 647.1833.

Macrocycle 41: To a solution of diol $39(56 \mathrm{mg}, 0.087 \mathrm{mmol})$ in $\mathrm{CH}_{2} \mathrm{Cl}_{2}(20 \mathrm{~mL}, 0.005 \mathrm{M})$ was added Grubbs II catalyst $(3.7 \mathrm{mg}, 0.05 \mathrm{mmol})$ at room temperature. The reaction mixture was

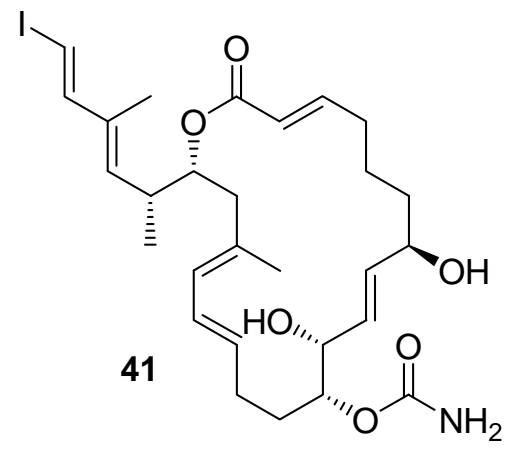
stirred for $1 \mathrm{~h}$ before it was concentrated in vacuo. Flash column chromatography (silica gel, hexanes:EtOAc: $\mathrm{Et}_{3} \mathrm{~N}$ 3:7:0.05 $\rightarrow$ EtOAc:MeOH:Et ${ }_{3} \mathrm{~N}$ 9.5:0.5:0.05) afforded macrocycle 41 (43 $\mathrm{mg}, 81 \%$ ) as a white gummy solid. $\mathbf{4 1}: R_{\mathrm{f}}=0.32$ (silica gel, hexanes:EtOAc 2:8); $[\alpha]_{\mathrm{D}}{ }^{25}=-9.5\left(\mathrm{CHCl}_{3}, c=0.31\right)$; IR (film) $v_{\max } 3362,2923,2851,1713,1391,1068,976 \mathrm{~cm}^{-1} ;{ }^{1} \mathrm{H} \mathrm{NMR}\left(600 \mathrm{MHz}, \mathrm{CDCl}_{3}\right): \delta=7.07(\mathrm{~d}, J$ $=14.6 \mathrm{~Hz}, 1 \mathrm{H}), 6.84(\mathrm{ddd}, J=15.4,10.0,5.2 \mathrm{~Hz}, 1 \mathrm{H}), 6.23(\mathrm{~d}, J=14.6 \mathrm{~Hz}, 1 \mathrm{H}), 6.10(\mathrm{dd}, J=$ $15.5,10.9 \mathrm{~Hz}, 1 \mathrm{H}), 5.68(\mathrm{dd}, J=15.5,7.8 \mathrm{~Hz}, 1 \mathrm{H}), 5.76(\mathrm{dd}, J=15.5,11.9 \mathrm{~Hz}, 1 \mathrm{H}), 5.74(\mathrm{~d}, J$ $=15.4 \mathrm{~Hz}, 1 \mathrm{H}), 5.65(\mathrm{~d}, J=10.8 \mathrm{~Hz}, 1 \mathrm{H}), 5.40(\mathrm{ddd}, J=14.5,9.6,4.7 \mathrm{~Hz}, 1 \mathrm{H}), 5.31(\mathrm{~d}, J=9.8$ $\mathrm{Hz}, 1 \mathrm{H}$ ), 4.98 (ddd, $J=11.3,7.8,2.1 \mathrm{~Hz}, 1 \mathrm{H}), 4.72-4.80$ (brs, $2 \mathrm{H}$ ), 4.70 (ddd, $J=13.0,5.2$, $4.7 \mathrm{~Hz}, 1 \mathrm{H}), 4.35(\mathrm{bm}, 1 \mathrm{H}), 4.06(\mathrm{dd}, J=16.0,4.0 \mathrm{~Hz}, 1 \mathrm{H}), 2.71(\mathrm{ddq}, J=11.3,10.8,6.8 \mathrm{~Hz}, 1$ H), 2.27-2.15 (m, 2 H), 2.16-2.09 (m, 2 H), 2.06 (dd, $J=14.0,11.6 \mathrm{~Hz}, 1 \mathrm{H}), 2.0$ (m, $1 \mathrm{H}), 1.74$ (s, $3 \mathrm{H}), 1.70(\mathrm{~m}, 3 \mathrm{H}), 1.67-1.58(\mathrm{~m}, 3 \mathrm{H}), 1.54-1.44$ (m, $5 \mathrm{H}), 0.99$ ppm (d, $J=6.8 \mathrm{~Hz}, 3 \mathrm{H})$; ${ }^{13} \mathrm{C}$ NMR $\left(150 \mathrm{MHz}, \mathrm{CDCl}_{3}\right): \delta=166.1,157.6,149.5,149.1,137.3,135.2,135.6,132.0,131.3$, 
$128.0,127.3,127.1,121.1,77.9,76.1,74.3,73.8,70.3,45.8,43.5,37.3,36.1,32.9,28.7,23.2$, 16.9, 16.3, 12.3 ppm; HRMS (ESI): calcd for $\mathrm{C}_{28} \mathrm{H}_{40} \mathrm{INO}_{6} \mathrm{Na}^{+}\left[\mathrm{M}+\mathrm{Na}^{+}\right] 636.1793$, found 636.1810.

Palmerolide isomer 1 and decarbamated palmerolide $A$ isomer 43: To a mixture of vinyl iodide 41 (10 mg, $0.016 \mathrm{mmol}$ ), amide $42(3.2 \mathrm{mg}, 0.033 \mathrm{mmol}), \mathrm{K}_{2} \mathrm{CO}_{3}(14 \mathrm{mg}, 0.098 \mathrm{mmol}$,

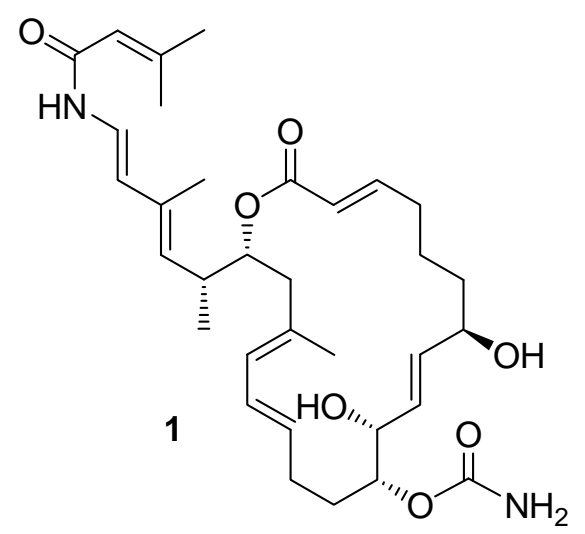
flame-dried) and CuI (4.6 mg, $0.024 \mathrm{mmol})$ in DMF (1.5 mL) was added $N, N^{\prime}$-dimethylethylenediamine $(5.4 \mu \mathrm{L}, 0.049$ mmol) at room temperature. The reaction mixture was stirred for $1 \mathrm{~h}$ before it was concentrated in vacuo. The resulting residue was diluted with EtOAc $(10 \mathrm{~mL})$, filtered through a pad of Celite ${ }^{\circledR}$, washed with EtOAc and concentrated in vacuo. Preparative TLC purification afforded $1(2.95 \mathrm{mg}, 31 \%)$ as a white gummy solid, and decarbamated product $43(0.44 \mathrm{mg}, 5 \%)$ as a white gummy solid. Palmerolide A isomer 1: $R_{\mathrm{f}}=$ 0.22 (silica gel, hexanes:EtOAc 0:1); $[\alpha]_{\mathrm{D}}^{25}=+36.0\left(\mathrm{CH}_{2} \mathrm{Cl}_{2}, c=0.030\right)$; IR (film) $v_{\max } 3390$, 2917, 1712, 1457, 1377, 1259, $1146 \mathrm{~cm}^{-1} ;{ }^{1} \mathrm{H}$ NMR $\left(600 \mathrm{MHz}\right.$, DMSO- $\left.d^{6}\right): \delta=9.91(\mathrm{~d}, J=10.4$ Hz, $1 \mathrm{H}), 6.85$ (dd, $J=14.8,10.4 \mathrm{~Hz}, 1 \mathrm{H}), 6.72$ (ddd, $J=15.2,8.4,6.4 \mathrm{~Hz}, 1 \mathrm{H}), 6.40$ (brs, $2 \mathrm{H})$, $6.10(\mathrm{dd}, J=14.4,10.8 \mathrm{~Hz}, 1 \mathrm{H}), 5.85$ ( d, $J=14.4 \mathrm{~Hz}, 1 \mathrm{H}), 5.76$ (d, $J=15.2 \mathrm{~Hz}, 1 \mathrm{H}), 5.69$ (brs, $1 \mathrm{H}), 5.60(\mathrm{~d}, J=9.6 \mathrm{~Hz}, 1 \mathrm{H}), 5.58(\mathrm{dd}, J=15.6,4.0 \mathrm{~Hz}, 1 \mathrm{H}), 5.49(\mathrm{dd}, J=15.6,6.4 \mathrm{~Hz}, 1 \mathrm{H})$, $5.39(\mathrm{ddd}, J=14.8,9.2,5.2 \mathrm{~Hz}, 1 \mathrm{H}), 4.92(\mathrm{~d}, J=3.6 \mathrm{~Hz}, 1 \mathrm{H}), 4.74(\mathrm{~d}, J=3.6 \mathrm{~Hz}, 1 \mathrm{H}), 4.85$ (ddd, $J=10.4,8.0,1.3 \mathrm{~Hz}, 1 \mathrm{H}), 4.48$ (ddd, $J=9.6,7.2,2.4 \mathrm{~Hz}, 1 \mathrm{H}), 4.04$ (brs, $1 \mathrm{H}), 3.89$ (m, 1 H), 2.69 (m, 1 H), 2.19-1.99 (m, 6 H), 2.12 (s, 3 H), 1.90 (m, 2 H), 1.83 (s, 3 H), 1.70 (s, 3 H), $1.40-1.29$ (m, $6 \mathrm{H}), 1.27-1.18$ (m, $4 \mathrm{H}), 0.90 \mathrm{ppm}(\mathrm{d}, J=6.4 \mathrm{~Hz}, 3 \mathrm{H}) ;{ }^{13} \mathrm{C}$ NMR $(150 \mathrm{MHz}$, 
DMSO- $\left.d^{6}\right): \delta=165.8,163.6,157.4,152.3,149.7,136.1,133.1,132.7,131.8,130.3,128.5,128.3$, $126.9,122.6,121.3,118.6,116.9,74.3,73.5,70.0,69.0,43.3,37.3,37.2,32.7,32.6,31.9,30.1$, 29.0, 23.9, 17.6, 16.7, 13.2 ppm; HRMS (ESI): calcd for $\mathrm{C}_{33} \mathrm{H}_{48} \mathrm{~N}_{2} \mathrm{O}_{7} \mathrm{Na}^{+}\left[\mathrm{M}+\mathrm{Na}^{+}\right] 607.3354$, found 607.3368 .

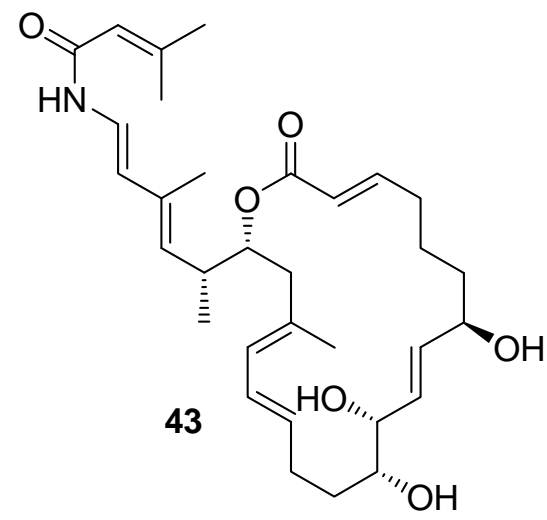

Decarbamated palmerolide A isomer 43: $\mathrm{R}_{\mathrm{f}}=0.31$ (silica gel, hexanes:EtOAc 1:4); $[\alpha]_{\mathrm{D}}{ }^{25}=-14.0(\mathrm{MeOH}, c=0.23)$; IR (film) $v_{\max } 3377,2927,1712,1641,1517,1446,1220,1075$, 1023, $971 \mathrm{~cm}^{-1} ;{ }^{1} \mathrm{H}$ NMR $\left(600 \mathrm{MHz}\right.$, DMSO- $\left.d^{6}\right): \delta=9.85(\mathrm{~d}$, $J=10.2 \mathrm{~Hz}, 1 \mathrm{H}), 6.85(\mathrm{dd}, J=14.4,10.2 \mathrm{~Hz}, 1 \mathrm{H}), 6.70(\mathrm{dt}, J$ $=15.0,8.4 \mathrm{~Hz}, 1 \mathrm{H}), 6.08(\mathrm{dd}, J=15.0,10.8 \mathrm{~Hz}, 1 \mathrm{H}), 5.85(\mathrm{~d}$, $J=14.4 \mathrm{~Hz}, 1 \mathrm{H}), 5.76(\mathrm{~d}, J=15.6 \mathrm{~Hz}, 1 \mathrm{H}), 5.69(\mathrm{~s}, 1 \mathrm{H}), 5.62(\mathrm{~d}, J=10.8 \mathrm{~Hz}, 1 \mathrm{H}), 5.53(\mathrm{dd}, J$ $=15.6,3.6 \mathrm{~Hz}, 1 \mathrm{H}), 5.49(\mathrm{dd}, J=15.6,3.6 \mathrm{~Hz}, 1 \mathrm{H}), 5.40(\mathrm{ddd}, J=15.0,10.2,4.8 \mathrm{~Hz}, 1 \mathrm{H})$, $5.14(\mathrm{~d}, J=9.6 \mathrm{~Hz}, 1 \mathrm{H}), 4.87(\mathrm{~m}, 1 \mathrm{H}), 4.67(\mathrm{~d}, J=4.8 \mathrm{~Hz}, 1 \mathrm{H}), 4.64(\mathrm{~d}, J=4.8 \mathrm{~Hz}, 1 \mathrm{H}), 4.48$ (d, $J=4.8 \mathrm{~Hz}, 1 \mathrm{H}), 4.05(\mathrm{bm}, 1 \mathrm{H}), 3.66(\mathrm{bm}, 1 \mathrm{H}), 3.16(\mathrm{~m}, 1 \mathrm{H}), 2.69(\mathrm{~m}, 1 \mathrm{H}), 2.28-1.94(\mathrm{~m}$, $6 \mathrm{H}), 2.12(\mathrm{~s}, 3 \mathrm{H}), 1.91-1.79(\mathrm{~m}, 1 \mathrm{H}), 1.83(\mathrm{~s}, 3 \mathrm{H}), 1.70(\mathrm{~s}, 3 \mathrm{H}), 1.62(\mathrm{~s}, 3 \mathrm{H}), 1.41-1.30(\mathrm{~m}$, $2 \mathrm{H}), 1.15-1.09(\mathrm{~m}, 1 \mathrm{H}), 0.90 \mathrm{ppm}(\mathrm{d}, J=6.6 \mathrm{~Hz}, 3 \mathrm{H}) ;{ }^{13} \mathrm{C}$ NMR $\left(150 \mathrm{MHz}\right.$, DMSO- $\left.d^{6}\right): \delta=$ $152.3,150.0,135.5,133.1,131.4,130.3,129.3,128.4,126.6,122.6,121.3,118.6,116.9,74.3$, 73.9, 69.7, 43.4, 37.2, 37.2, 33.9, 32.7, 29.8, 27.5, 23.8, 20.1, 17.6, 16.7, 13.1 ppm; HRMS (ESI): calcd for $\mathrm{C}_{32} \mathrm{H}_{47} \mathrm{NO}_{6} \mathrm{Na}^{+}\left[\mathrm{M}+\mathrm{Na}^{+}\right] 564.3296$, found 564.3310

Macrocycle 44: To a solution of cyclic carbonate 40 (13.0 mg, $20.9 \mu \mathrm{mol})$ in $\mathrm{CH}_{2} \mathrm{Cl}_{2}(4.8 \mathrm{~mL}$, $0.005 \mathrm{M})$ was added Grubbs II catalyst $(3.6 \mathrm{mg}, 4.18 \mu \mathrm{mol})$ at room tempertature. The reaction mixture was stirred for $4 \mathrm{~h}$ before it was concentrated in vacuo. Preparative TLC purification 


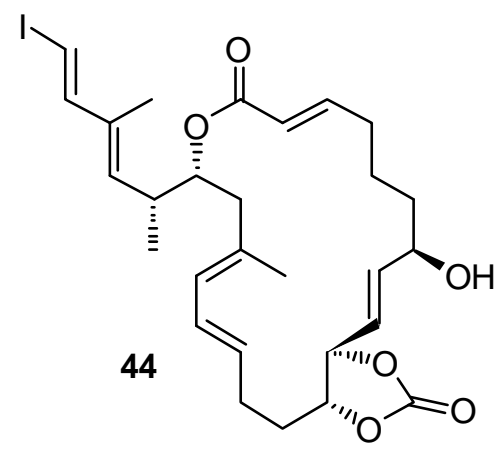

(silica gel, hexanes:EtOAc 1:1) afforded macrocycle $\mathbf{4 4}(9.0 \mathrm{mg}$, $72 \%$ ) as a colorless oil. $44: R_{\mathrm{f}}=0.30$ (silica gel, hexanes:EtOAc $7: 3) ;[\alpha]_{\mathrm{D}}^{25}=+9.7\left(\mathrm{CHCl}_{3}, c=0.74\right) ; \mathrm{IR}($ film $) v_{\max } 3376,2923$, $1801,1714,1457,1268,1159,1023,773 \mathrm{~cm}^{-1} ;{ }^{1} \mathrm{H}$ NMR $(600$ $\left.\mathrm{MHz}, \mathrm{CDCl}_{3}\right): \delta=7.07(\mathrm{~d}, J=14.6 \mathrm{~Hz}, 1 \mathrm{H}), 6.88(\mathrm{ddd}, J=14.7$, 7.6, $6.3 \mathrm{~Hz}, 1 \mathrm{H}), 6.23(\mathrm{~m}, 2 \mathrm{H}), 5.89(\mathrm{dd}, J=15.5,5.0 \mathrm{~Hz}, 1 \mathrm{H}), 5.74(\mathrm{dd}, J=15.5,6.5 \mathrm{~Hz}, 1 \mathrm{H})$, $5.73(\mathrm{~d}, J=14.5 \mathrm{~Hz}, 1 \mathrm{H}), 5.34(\mathrm{ddd}, J=14.9,10.1,4.8 \mathrm{~Hz}, 1 \mathrm{H}), 5.32(\mathrm{~d}, J=10.0 \mathrm{~Hz}, 1 \mathrm{H})$, $5.00(\mathrm{ddd}, J=10.9,7.2,1.5 \mathrm{~Hz}, 1 \mathrm{H}), 4.66(\mathrm{t}, J=7.0 \mathrm{~Hz}, 1 \mathrm{H}), 4.31(\mathrm{dd}, J=10.6,5.2 \mathrm{~Hz}, 1 \mathrm{H})$, 4.15 (ddd, $J=10.2,7.4,2.8 \mathrm{~Hz}, 1 \mathrm{H}), 3.66$ (s, $1 \mathrm{H}), 2.77$ (m, $1 \mathrm{H}), 2.37$ (m, $1 \mathrm{H}), 2.30-2.10$ (m, $6 \mathrm{H}), 2.00-1.93(\mathrm{~m}, 2 \mathrm{H}), 1.73(\mathrm{~s}, 3 \mathrm{H}), 1.72(\mathrm{~s}, 3 \mathrm{H}), 1.68-1.52(\mathrm{~m}, 4 \mathrm{H}), 1.00 \mathrm{ppm}(\mathrm{d}, J=6.6$ $\mathrm{Hz}, 3 \mathrm{H}) ;{ }^{13} \mathrm{C} \mathrm{NMR}\left(150 \mathrm{MHz}, \mathrm{CDCl}_{3}\right): \delta=166.0,154.2,149.5,148.6,138.0,135.2,135.0$ $133.4,129.3,129.2,127.4,124.2,121.4,81.7,80.8,74.4,74.1,70.4,42.9,37.1,35.4,33.0,32.0$, 28.2, 23.1, 16.9, 16.4, 12.4 ppm; HRMS (ESI): calcd for $\mathrm{C}_{28} \mathrm{H}_{37} \mathrm{IO}_{6} \mathrm{Na}^{+}\left[\mathrm{M}+\mathrm{Na}^{+}\right] 619.1527$, found 619.1542

Triol 45: A solution of cyclic carbonate $44(9.0 \mathrm{mg}, 15.1 \mu \mathrm{mol})$ in a mixture of $\mathrm{Et}_{3} \mathrm{~N} / \mathrm{MeOH} / \mathrm{H}_{2} \mathrm{O}(1: 5: 1,2.0 \mathrm{~mL})$ was heated at $40{ }^{\circ} \mathrm{C}$ for $4 \mathrm{~h}$. The reaction mixture was cooled

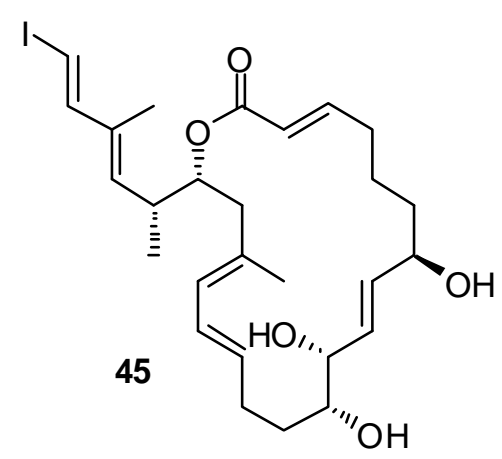
to room temperature before it was concentrated in vacuo. Preparative TLC purification (silica gel, hexanes:EtOAc 1:9) afforded triol $45(4.9 \mathrm{mg}, 57 \%)$ as a gummy solid. $45: R_{\mathrm{f}}=0.45$ (silica gel, hexanes:EtOAc 0:1); $[\alpha]_{\mathrm{D}}{ }^{25}=-27.5\left(\mathrm{CHCl}_{3}, c=0.38\right)$; IR (film) $v_{\max } 3379,2926,1715,1653,1448,1266,1163,975$ $\mathrm{cm}^{-1} ;{ }^{1} \mathrm{H}$ NMR $\left(600 \mathrm{MHz}, \mathrm{CDCl}_{3}\right): \delta=7.07(\mathrm{~d}, J=14.7 \mathrm{~Hz}, 1 \mathrm{H}), 6.85(\mathrm{ddd}, J=15.1,9.7,5.1$ 
$\mathrm{Hz}, 1 \mathrm{H}), 6.22(\mathrm{~d}, J=14.7 \mathrm{~Hz}, 1 \mathrm{H}), 6.16(\mathrm{dd}, J=14.6,10.8 \mathrm{~Hz}, 1 \mathrm{H}), 5.74(\mathrm{~d}, J=15.4 \mathrm{~Hz}, 1 \mathrm{H})$, $5.74(\mathrm{bm}, 1 \mathrm{H}), 5.67(\mathrm{~d}, J=10.6 \mathrm{~Hz}, 1 \mathrm{H}), 5.66(\mathrm{bm}, 1 \mathrm{H}), 5.42(\mathrm{ddd}, J=14.5,10.0,4.1 \mathrm{~Hz}, 1 \mathrm{H})$, $5.31(\mathrm{~d}, J=9.9 \mathrm{~Hz}, 1 \mathrm{H}), 4.98$ (ddd, $J=10.0,7.8,1.9 \mathrm{~Hz}, 1 \mathrm{H}), 4.37$ (brs, $1 \mathrm{H}), 3.86$ (brs, $1 \mathrm{H}$ ), $3.66(\mathrm{~s}, 1 \mathrm{H}), 3.43$ (brs, $1 \mathrm{H}), 2.71(\mathrm{~m}, 1 \mathrm{H}), 2.38(\mathrm{~m}, 1 \mathrm{H}), 2.27-1.60(\mathrm{~m}, 6 \mathrm{H}), 1.74(\mathrm{~s}, 3 \mathrm{H})$, $1.71(\mathrm{~s}, 3 \mathrm{H}), 1.69-1.60(\mathrm{~m}, 1 \mathrm{H}), 1.50(\mathrm{~m}, 2 \mathrm{H}), 1.38-1.21(\mathrm{~m}, 4 \mathrm{H}), 0.99 \mathrm{ppm}(\mathrm{d}, J=6.7 \mathrm{~Hz}, 3$ $\mathrm{H}) ;{ }^{13} \mathrm{C}$ NMR $\left(150 \mathrm{MHz}, \mathrm{CDCl}_{3}\right): \delta=166.2,149.6,149.1,137.0,135.2,135.0,132.2,131.8$, $128.1,127.8,127.1,121.2,74.3,74.2,73.9,70.3,45.9,43.6,37.3,36.1,32.9,29.3,23.1,16.9$, 16.3, 12.4, 8.7 ppm; HRMS (ESI): calcd for $\mathrm{C}_{27} \mathrm{H}_{39} \mathrm{IO}_{5} \mathrm{Na}^{+}\left[\mathrm{M}+\mathrm{Na}^{+}\right]$593.1734, found 593.1726.

Enamide 43: To a mixture of iodide 45 (11.0 mg, $19.2 \mu \mathrm{mol})$, amide 42 (3.8 mg, $38.5 \mu \mathrm{mol})$, $\mathrm{K}_{2} \mathrm{CO}_{3}(13.5 \mathrm{mg}, 96.4 \mu \mathrm{mol})$ and $\mathrm{CuI}(5.5 \mathrm{mg}, 28.9 \mu \mathrm{mol})$ in $\mathrm{DMF}(1.0 \mathrm{~mL})$ was added $N, N^{\prime}-$

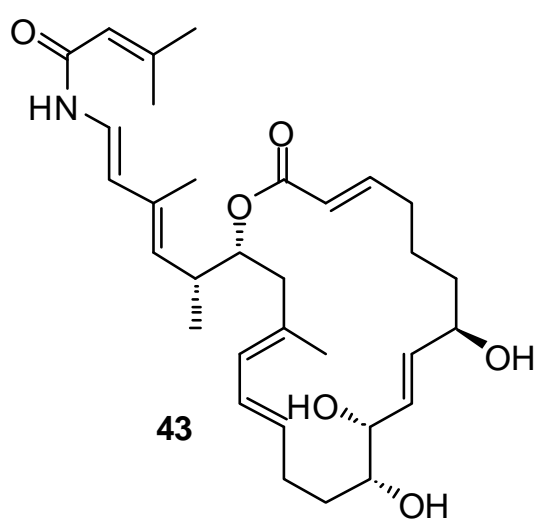
dimethyethylenediamine $(6.4 \mu \mathrm{L}, 57.8 \mu \mathrm{mol})$ at room temperature. The reaction mixture was stirred for $1 \mathrm{~h}$ before it was concentrated in vacuo. The resulting residue was diluted with EtOAc $(10 \mathrm{~mL})$, filtered through a pad of Celite $^{\circledR}$, washed with EtOAc $(10 \mathrm{~mL})$ and concentrated in vacuo. Preparative TLC purification (silica gel, EtOAc: $\mathrm{Et}_{3} \mathrm{~N}$ 100:1) afforded enamide $43(4.5 \mathrm{mg}, 45 \%)$ as a gummy solid. All physical properties of this compound were identical to those reported for decarbamated product $\mathbf{4 3}$ obtained from vinyl iodide 41 .

Tetraene 46: To a mixture of vinyl stannane 3 (175 mg, $0.338 \mathrm{mmol})$, vinyl iodide 19-epi-2 (110 mg, $0.259 \mathrm{mmol}), \mathrm{Pd}(\mathrm{dba})_{2}(45 \mathrm{mg}, 0.078 \mathrm{mmol}), \mathrm{AsPh}_{3}(160 \mathrm{mg}, 0.518 \mathrm{mmol})$ and $\mathrm{LiCl}$ 


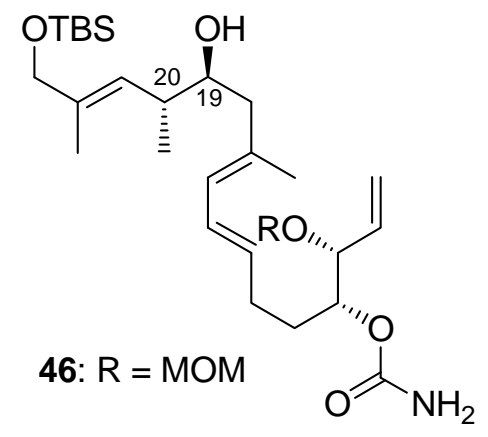

(32 mg, $0.78 \mathrm{mmol}$, freshly dried by heating with a heatgun for 20 min under vacuum) was added NMP $(2.5 \mathrm{~mL}$, degassed by using the freeze-thaw process sequence three times) at room temperature. The reaction mixture was stirred for $12 \mathrm{~h}$ before it was quenched with water $(10 \mathrm{~mL})$. The resulting mixture was extracted with EtOAc $(3 \times 30 \mathrm{~mL})$, and the combined organic layers were dried $\left(\mathrm{Na}_{2} \mathrm{SO}_{4}\right)$ and concentrated in vacuo. Flash column chromatography (silica gel, hexanes:EtOAc:Et ${ }_{3} \mathrm{~N}$ 3:2:0.01) afforded tetraene $46(83 \mathrm{mg}, 61 \%)$ as a pale yellow oil. $46: R_{\mathrm{f}}=0.30$ (silica gel, hexanes:EtOAc 3:2); $[\alpha]_{\mathrm{D}}^{25}=-32.6\left(\mathrm{CH}_{2} \mathrm{Cl}_{2}, c=0.90\right)$; IR (film) $v_{\max } 3357,2955,2929,2856,1716,1603,1463$, 1387, 1256, 1032, $836 \mathrm{~cm}^{-1} ;{ }^{1} \mathrm{H} \mathrm{NMR}\left(600 \mathrm{MHz}, \mathrm{CDCl}_{3}\right): \delta=6.27(\mathrm{dd}, J=15.0,10.8 \mathrm{~Hz}, 1 \mathrm{H})$, $5.86(\mathrm{~d}, J=10.8 \mathrm{~Hz}, 1 \mathrm{H}), 5.72(\mathrm{ddd}, J=17.4,10.2,7.2 \mathrm{~Hz}, 1 \mathrm{H}), 5.58(\mathrm{dt}, J=15.6,7.8 \mathrm{~Hz}, 1 \mathrm{H})$, $5.33(\mathrm{brd}, J=10.2 \mathrm{~Hz}, 1 \mathrm{H}), 5.32$ (brd, $J=17.4 \mathrm{~Hz}, 1 \mathrm{H}), 5.31(\mathrm{~d}, J=9.6 \mathrm{~Hz}, 1 \mathrm{H}), 4.88$ (dt, $J=$ 7.2, $5.4 \mathrm{~Hz}, 1 \mathrm{H}), 4.81$ (brs, $2 \mathrm{H}), 4.70(\mathrm{~d}, J=6.6 \mathrm{~Hz}, 1 \mathrm{H}), 4.58(\mathrm{~d}, J=6.6 \mathrm{~Hz}, 1 \mathrm{H}), 4.11(\mathrm{dd}, J$ $=7.8,4.2 \mathrm{~Hz}, 1 \mathrm{H}), 4.04(\mathrm{~s}, 2 \mathrm{H}), 3.57$ (ddd, $J=12.6,5.4,2.4 \mathrm{~Hz}, 1 \mathrm{H}), 3.39$ (s, $3 \mathrm{H}), 2.50$ (ddq, $J=10.2,6.6,6.6 \mathrm{~Hz}, 1 \mathrm{H}), 2.24(\mathrm{~d}, J=13.8 \mathrm{~Hz}, 1 \mathrm{H}), 2.18(\mathrm{q}, J=7.8 \mathrm{~Hz}, 2 \mathrm{H}), 2.05(\mathrm{dd}, J=$ 13.2, 10.2 Hz, $1 \mathrm{H}), 1.84$ (brs, $1 \mathrm{H}), 1.76$ (s, $3 \mathrm{H}), 1.75-1.69$ (m, $2 \mathrm{H}), 1.63$ (s, $3 \mathrm{H}), 1.01$ (d, $J=$ $6.6 \mathrm{~Hz}, 3 \mathrm{H}), 0.92(\mathrm{~s}, 9 \mathrm{H}), 0.07 \mathrm{ppm}(\mathrm{s}, 6 \mathrm{H}) ;{ }^{13} \mathrm{C} \mathrm{NMR}\left(150 \mathrm{MHz}, \mathrm{CDCl}_{3}\right): \delta=156.6,136.2$, 134.0, 133.5, 131.8, 127.6, 127.0, 125.9, 119.4, 94.0, 77.9, 75.3, 72.8, 68.5, 55.7, 44.8, 37.8, 30.5, 28.8, 26.0, 18.4, 16.9, 16.6, 13.8, -5.2 ppm; HRMS (ESI): calcd for $\mathrm{C}_{28} \mathrm{H}_{51} \mathrm{NO}_{6} \mathrm{SiNa}^{+}\left[\mathrm{M}+\mathrm{Na}^{+}\right]$ 548.3378, found 548.3409.

Tetraene 47: To a mixture of vinyl stannane 3 (515 mg, 0.99 mmol), vinyl iodide 20-epi-2 (320 $\mathrm{mg}, 0.755 \mathrm{mmol}), \mathrm{Pd}(\mathrm{dba})_{2}(130 \mathrm{mg}, 0.227 \mathrm{mmol}), \mathrm{AsPh}_{3}(462 \mathrm{mg}, 1.51 \mathrm{mmol})$ and $\mathrm{LiCl}(93 \mathrm{mg}$, 


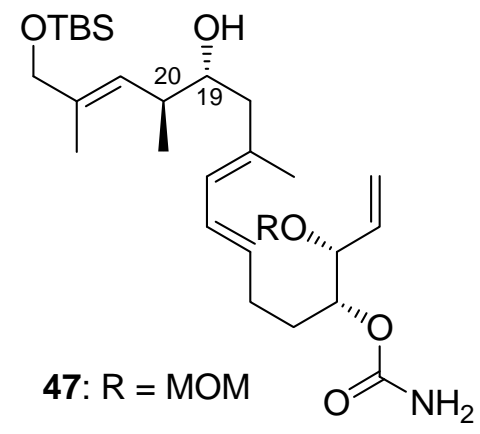

$2.27 \mathrm{mmol}$, freshly dried by heating with a heatgun for $20 \mathrm{~min}$ under vacuum) was added NMP $(8.0 \mathrm{~mL}$, degassed by using the freeze-thaw process sequence three times) at room temperature. The reaction mixture was stirred for $12 \mathrm{~h}$ before it was quenched with water $(10 \mathrm{~mL})$. The resulting mixture was extracted with EtOAc $(3 \times 30 \mathrm{~mL})$, and the combined organic layers were dried $\left(\mathrm{Na}_{2} \mathrm{SO}_{4}\right)$ and concentrated in vacuo. Flash column chromatography (silica gel, hexanes:EtOAc:Et ${ }_{3} \mathrm{~N}$ 3:2:0.01) afforded tetraene $47(250 \mathrm{mg}, 63 \%)$ as a pale yellow oil. $47: R_{\mathrm{f}}=0.32$ (silica gel, hexanes:EtOAc 3:2); $[\alpha]_{\mathrm{D}}^{25}=-17.7\left(\mathrm{CH}_{2} \mathrm{Cl}_{2}, c=0.40\right) ;$ IR (film) $v_{\max } 3354,2956,2929,2857,1717,1604,1388$ 1321, 1252, 1033, $837 \mathrm{~cm}^{-1} ;{ }^{1} \mathrm{H} \mathrm{NMR}\left(600 \mathrm{MHz}, \mathrm{CDCl}_{3}\right): \delta=6.27(\mathrm{dd}, J=15.0,10.8 \mathrm{~Hz}, 1 \mathrm{H})$, $5.86(\mathrm{~d}, J=10.8 \mathrm{~Hz}, 1 \mathrm{H}), 5.72(\mathrm{ddd}, J=17.4,10.8,7.2 \mathrm{~Hz}, 1 \mathrm{H}), 5.59(\mathrm{dt}, J=15.0,7.2 \mathrm{~Hz}, 1 \mathrm{H})$, $5.33(\mathrm{brd}, J=10.2 \mathrm{~Hz}, 1 \mathrm{H}), 5.32$ (brd, $J=17.4 \mathrm{~Hz}, 1 \mathrm{H}), 5.31$ (d, $J=10.2 \mathrm{~Hz}, 1 \mathrm{H}), 4.89$ (ddd, $J$ $=7.8,5.4,2.4 \mathrm{~Hz}, 1 \mathrm{H}), 4.81$ (brs, $2 \mathrm{H}), 4.70(\mathrm{~d}, J=6.6 \mathrm{~Hz}, 1 \mathrm{H}), 4.58(\mathrm{~d}, J=7.2 \mathrm{~Hz}, 1 \mathrm{H}), 4.12$ (dd, $J=7.2,4.8 \mathrm{~Hz}, 1 \mathrm{H}), 4.05$ (s, $2 \mathrm{H}), 3.58$ (ddd, $J=15.6,5.4,3.0 \mathrm{~Hz}, 1 \mathrm{H}), 3.40$ (s, $3 \mathrm{H}), 2.51$ $(\mathrm{dqd}, J=10.2,6.6,5.4 \mathrm{~Hz}, 1 \mathrm{H}), 2.24(\mathrm{~d}, J=13.8 \mathrm{~Hz}, 1 \mathrm{H}), 2.17$ (pent, $J=7.8 \mathrm{~Hz}, 2 \mathrm{H}), 2.05$ (dd, $J=13.8,9.6 \mathrm{~Hz}, 1 \mathrm{H}), 1.81(\mathrm{~d}, J=1.2 \mathrm{~Hz}, 1 \mathrm{H}), 1.77$ (s, $3 \mathrm{H}), 1.75-1.69$ (m, $2 \mathrm{H}), 1.63(\mathrm{~s}, 3 \mathrm{H})$, $1.01(\mathrm{~d}, J=6.6 \mathrm{~Hz}, 3 \mathrm{H}), 0.91(\mathrm{~s}, 9 \mathrm{H}), 0.08 \mathrm{ppm}(\mathrm{s}, 6 \mathrm{H}) ;{ }^{13} \mathrm{C} \mathrm{NMR}\left(150 \mathrm{MHz}, \mathrm{CDCl}_{3}\right): \delta=$ 156.6, 136.2, 134.0, 133.6, 131.8, 127.5, 127.0, 125.9, 119.4, 94.0, 77.8, 75.3, 72.8, 68.6, 55.7, $44.8,37.8,30.5,28.7,25.9,18.4,16.9,16.6,13.8,-5.2 \mathrm{ppm}$; HRMS (ESI): calcd for $\mathrm{C}_{28} \mathrm{H}_{51} \mathrm{NO}_{6} \mathrm{SiNa}^{+}\left[\mathrm{M}+\mathrm{Na}^{+}\right] 548.3378$, found 548.3414.

Tetraene 48: To a mixture of vinyl stannane 3 (346 mg, $0.66 \mathrm{mmol})$ and vinyl iodide ent-2 (218 $\mathrm{mg}, 0.51 \mathrm{mmol}), \mathrm{Pd}(\mathrm{dba})_{2}(87 \mathrm{mg}, 0.17 \mathrm{mmol}), \mathrm{AsPh}_{3}(312 \mathrm{mg}, 1.02 \mathrm{mmol})$ and $\mathrm{LiCl}(63 \mathrm{mg}$, 


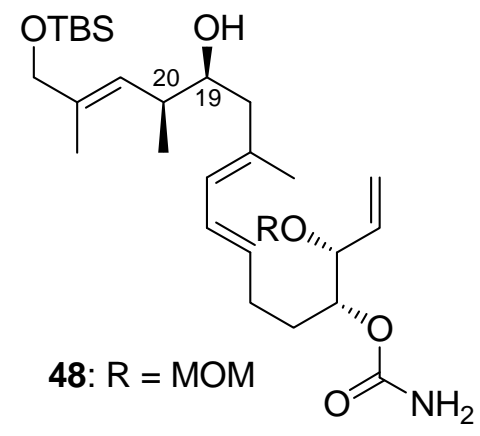

$1.7 \mathrm{mmol}$, freshly dried by heating with a heatgun for 20 min under vacuum $)$ was added NMP $(6.0 \mathrm{~mL}$, degassed by using the freezethaw process sequence three times) at room temperature. The reaction mixture was stirred for $12 \mathrm{~h}$ before it was quenched with water $(10 \mathrm{~mL})$. The resulting mixture was extracted with EtOAc (3

$\times 30 \mathrm{~mL})$, and the combined organic layers were dried $\left(\mathrm{Na}_{2} \mathrm{SO}_{4}\right)$ and concentrated in vacuo.

Flash column chromatography (silica gel, hexanes:EtOAc:Et ${ }_{3} \mathrm{~N}$ 3:2:0.01) afforded tetraene 48 (135 mg, 50\%) as a pale yellow oil. 48: $R_{\mathrm{f}}=0.30$ (silica gel, hexanes:EtOAc 3:2); $[\alpha]_{\mathrm{D}}{ }^{25}=-36.0$ $\left(\mathrm{CH}_{2} \mathrm{Cl}_{2}, c=0.27\right)$; IR (film) $v_{\max } 3356,2955,2929,2857,1737,1603,1442,1389,1257,1032$ $837 \mathrm{~cm}^{-1} ;{ }^{1} \mathrm{H}$ NMR $\left(600 \mathrm{MHz}, \mathrm{CDCl}_{3}\right): \delta=6.26(\mathrm{dd}, J=15.0,10.8 \mathrm{~Hz}, 1 \mathrm{H}), 5.86(\mathrm{~d}, J=10.8$ $\mathrm{Hz}, 1 \mathrm{H}), 5.72$ (ddd, $J=17.4,10.2,6.6 \mathrm{~Hz}, 1 \mathrm{H}), 5.59$ (dt, $J=15.0,6.6 \mathrm{~Hz}, 1 \mathrm{H}), 5.32$ (brd, $J=$ $9.6 \mathrm{~Hz}, 1 \mathrm{H}), 5.31$ (brd, $J=17.4 \mathrm{~Hz}, 1 \mathrm{H}), 5.26(\mathrm{~d}, J=9.6 \mathrm{~Hz}, 1 \mathrm{H}), 4.89$ (dt, $J=7.8,4.8 \mathrm{~Hz}, 1$ H), 4.84 (brs, $2 \mathrm{H}), 4.70(\mathrm{~d}, J=6.6 \mathrm{~Hz}, 1 \mathrm{H}), 4.58(\mathrm{~d}, J=7.2 \mathrm{~Hz}, 1 \mathrm{H}), 4.12(\mathrm{dd}, J=10.8,6.0 \mathrm{~Hz}$, $1 \mathrm{H}), 4.02$ (s, $2 \mathrm{H}$ ), 3.50 (ddd, $J=10.2,6.0,3.0 \mathrm{~Hz}, 1 \mathrm{H}), 3.39$ (s, $3 \mathrm{H}), 2.46$ (ddq, $J=9.6,7.2$, $6.6 \mathrm{~Hz}, 1 \mathrm{H}), 2.31(\mathrm{~d}, J=13.2 \mathrm{~Hz}, 1 \mathrm{H}), 2.21-2.14(\mathrm{~m}, 2 \mathrm{H}), 1.94(\mathrm{dd}, J=13.8,10.2 \mathrm{~Hz}, 1 \mathrm{H})$, 1.87 (s, $1 \mathrm{H}), 1.79-1.69$ (m, $2 \mathrm{H}), 1.76$ (s, $3 \mathrm{H}), 1.67$ (s, $3 \mathrm{H}), 1.04$ (d, J=6.6 Hz, $3 \mathrm{H}), 0.91$ (s, 9 $\mathrm{H}), 0.07 \mathrm{ppm}(\mathrm{s}, 6 \mathrm{H}) ;{ }^{13} \mathrm{C} \mathrm{NMR}\left(150 \mathrm{MHz}, \mathrm{CDCl}_{3}\right): \delta=156.6,135.0,134.0,133.6,131.9,127.7$, $126.9,126.6,119.4,94.0,77.9,75.3,73.0,68.4,55.7,45.6,38.3,30.5,28.8,25.9,18.4,16.7$, 16.5, 13.8, -5.2 ppm; HRMS (ESI): calcd for $\mathrm{C}_{28} \mathrm{H}_{51} \mathrm{NO}_{6} \mathrm{SiNa}^{+}\left[\mathrm{M}+\mathrm{Na}^{+}\right] 548.3378$, found 548.3415.

Ester 49: To a solution of acid $4(40 \mathrm{mg}, 0.186 \mathrm{mmol})$ and $\mathrm{Et}_{3} \mathrm{~N}(0.086 \mathrm{~mL}, 0.62 \mathrm{mmol})$ in toluene $(0.6 \mathrm{~mL})$ was added 2,4,6-trichlorobenzoyl chloride $(29 \mu \mathrm{L}, 0.186 \mathrm{mmol})$ at room 


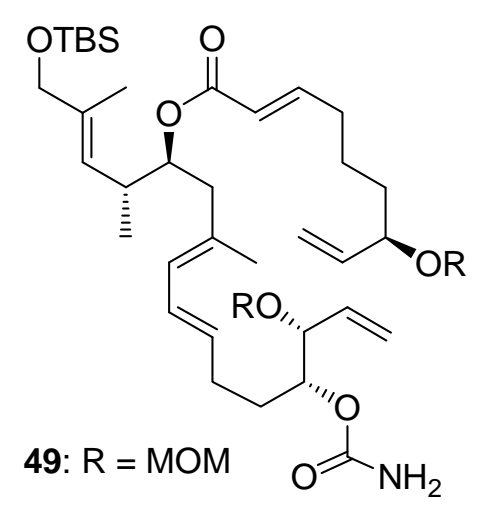

temperature. The reaction mixture was stirred for $1 \mathrm{~h}$ before a solution of alcohol 46 (65 mg, $0.124 \mathrm{mmol})$ and 4-DMAP (15 mg, $0.124 \mathrm{mmol})$ in toluene $(1.0 \mathrm{~mL})$ was added at room temperature.

The reaction mixture was stirred for $12 \mathrm{~h}$ before it was quenched with $\mathrm{NH}_{4} \mathrm{Cl}(15 \mathrm{~mL}$, sat. aq.). The resulting mixture was extracted with EtOAc $(3 \times 30 \mathrm{~mL})$, and the combined organic layers were dried $\left(\mathrm{Na}_{2} \mathrm{SO}_{4}\right)$ and concentrated in vacuo. Flash column chromatography (silica gel, hexanes:EtOAc:Et ${ }_{3} \mathrm{~N}$ 4:2:0.01) afforded ester $49(56 \mathrm{mg}, 63 \%)$ as a colorless oil. 49: $R_{\mathrm{f}}=0.35$ (silica gel, hexanes:EtOAc 3:2); $[\alpha]_{\mathrm{D}}{ }^{25}=+0.9\left(\mathrm{CH}_{2} \mathrm{Cl}_{2}, c=0.32\right.$ ); IR (film) $v_{\max } 3364,2930$, $2857,1718,1603,1463,1387,1318,1256,1150,1067,837 \mathrm{~cm}^{-1} ;{ }^{1} \mathrm{H}$ NMR $\left(600 \mathrm{MHz}, \mathrm{CDCl}_{3}\right)$ : $\delta=6.90(\mathrm{dt}, J=15.6,7.6 \mathrm{~Hz}, 1 \mathrm{H}), 6.20(\mathrm{dd}, J=15.0,10.8 \mathrm{~Hz}, 1 \mathrm{H}), 5.80(\mathrm{~d}, J=15.6 \mathrm{~Hz}, 1 \mathrm{H})$ $5.74(\mathrm{~d}, J=10.2 \mathrm{~Hz}, 1 \mathrm{H}), 5.70$ (ddd, $J=17.4,10.2,7.2 \mathrm{~Hz}, 1 \mathrm{H}), 5.66$ (ddd, $J=17.4,10.2,7.8$ $\mathrm{Hz}, 1 \mathrm{H}), 5.50(\mathrm{dt}, J=15.0,7.2 \mathrm{~Hz}, 1 \mathrm{H}), 5.37(\mathrm{~d}, J=10.2 \mathrm{~Hz}, 1 \mathrm{H}), 5.32(\mathrm{brd}, J=10.2 \mathrm{~Hz}, 1 \mathrm{H})$, 5.31 (brd, $J=17.4 \mathrm{~Hz}, 1 \mathrm{H}), 5.21$ (brd, $J=10.8 \mathrm{~Hz}, 1 \mathrm{H}), 5.20$ (brd, $J=17.4 \mathrm{~Hz}, 1 \mathrm{H}), 4.95$ (dt, $J$ $=7.8,4.8 \mathrm{~Hz}, 1 \mathrm{H}), 4.88(\mathrm{dt}, J=9.0,4.5 \mathrm{~Hz}, 1 \mathrm{H}), 4.79$ (brs, $2 \mathrm{H}), 4.70(\mathrm{~d}, J=6.6 \mathrm{~Hz}, 1 \mathrm{H}), 4.69$ $(\mathrm{d}, J=6.6 \mathrm{~Hz}, 1 \mathrm{H}), 4.58(\mathrm{~d}, J=7.2 \mathrm{~Hz}, 1 \mathrm{H}), 4.53(\mathrm{~d}, J=6.6 \mathrm{~Hz}, 1 \mathrm{H}), 4.10(\mathrm{dd}, J=7.2,5.4 \mathrm{~Hz}$, $1 \mathrm{H}), 4.02$ (s, $2 \mathrm{H}), 3.99$ (q, $J=6.6 \mathrm{~Hz}, 1 \mathrm{H}), 3.39$ (s, $3 \mathrm{H}), 3.37$ (s, $3 \mathrm{H}), 2.66$ (dqd, $J=10.8,7.2$, $6.6 \mathrm{~Hz}, 1 \mathrm{H}), 2.26-2.12(\mathrm{~m}, 6 \mathrm{H}), 1.76-1.48(\mathrm{~m}, 6 \mathrm{H}), 1.73(\mathrm{~s}, 3 \mathrm{H}), 1.59(\mathrm{~s}, 3 \mathrm{H}), 0.96$ (d, $J=$ $7.2 \mathrm{~Hz}, 3 \mathrm{H}), 0.92(\mathrm{~s}, 9 \mathrm{H}), 0.07 \mathrm{ppm}(\mathrm{s}, 6 \mathrm{H}) ;{ }^{13} \mathrm{C} \mathrm{NMR}\left(150 \mathrm{MHz}, \mathrm{CDCl}_{3}\right): \delta=166.3,156.5$, $148.5,138.1,135.7,134.0,132.5,131.4,127.5,127.2,124.6,121.7,119.4,117.4,94.0,93.7$, $77.8,77.0,75.4,75.2,68.3,55.7,55.4,42.4,35.3,34.9,32.0,30.4,28.7,25.9,23.8,18.4,17.1$, 16.6, 13.6, -5.2, -5.2 ppm; HRMS (ESI): calcd for $\mathrm{C}_{39} \mathrm{H}_{67} \mathrm{NO}_{9} \mathrm{SiNa}^{+}\left[\mathrm{M}+\mathrm{Na}^{+}\right]$744.4477, found 744.4499. 
Ester 50: To a solution of acid $4(114 \mathrm{mg}, 0.53 \mathrm{mmol})$ and $\mathrm{Et}_{3} \mathrm{~N}(0.20 \mathrm{~mL}, 1.33 \mathrm{mmol})$ in toluene $(1.5 \mathrm{~mL})$ was added 2,4,6-trichlorobenzoyl chloride $(90 \mu \mathrm{L}, 0.58 \mathrm{mmol})$ at room

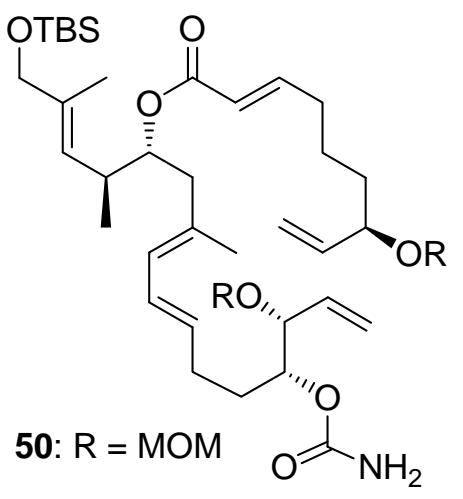
temperature. The reaction mixture was stirred for $1 \mathrm{~h}$ before a solution of alcohol $47(140 \mathrm{mg}, 0.267 \mathrm{mmol})$ and 4-DMAP $(33 \mathrm{mg}$, $0.267 \mathrm{mmol})$ in toluene $(1.5 \mathrm{~mL})$ was added at room temperature. The reaction mixture was stirred for $12 \mathrm{~h}$ before it was quenched with $\mathrm{NH}_{4} \mathrm{Cl}(15 \mathrm{~mL}$, sat. aq.). The resulting mixture was extracted with EtOAc $(3 \times 30 \mathrm{~mL})$, and the combined organic layers were dried $\left(\mathrm{Na}_{2} \mathrm{SO}_{4}\right)$ and concentrated in vacuo. Flash column chromatography (silica gel, hexanes:EtOAc: $\mathrm{Et}_{3} \mathrm{~N}$ 4:2:0.01) afforded ester $50(143 \mathrm{mg}, 74 \%)$ as a colorless oil. 50: $R_{\mathrm{f}}=0.35$ (silica gel, hexanes:EtOAc 3:2); $[\alpha]_{\mathrm{D}}{ }^{25}=+17.2\left(\mathrm{CH}_{2} \mathrm{Cl}_{2}, c=0.60\right)$; IR (film) $v_{\max } 3361,2930$, $2857,1718,1654,1442,1386,1258,1097,837 \mathrm{~cm}^{-1} ;{ }^{1} \mathrm{H} \mathrm{NMR}\left(600 \mathrm{MHz}, \mathrm{CDCl}_{3}\right): \delta=6.90(\mathrm{dt}$, $J=15.6,6.9 \mathrm{~Hz}, 1 \mathrm{H}), 6.19(\mathrm{dd}, J=15.0,10.8 \mathrm{~Hz}, 1 \mathrm{H}), 5.78(\mathrm{~d}, J=15.6 \mathrm{~Hz}, 1 \mathrm{H}), 5.72(\mathrm{~d}, J=$ $10.2 \mathrm{~Hz}, 1 \mathrm{H}), 5.70$ (ddd, $J=17.4,10.2,7.2 \mathrm{~Hz}, 1 \mathrm{H}), 5.65(\mathrm{ddd}, J=17.4,10.2,7.8 \mathrm{~Hz}, 1 \mathrm{H})$, $5.52(\mathrm{dt}, J=15.0,7.2 \mathrm{~Hz}, 1 \mathrm{H}), 5.36(\mathrm{~d}, J=10.2 \mathrm{~Hz}, 1 \mathrm{H}), 5.31(\mathrm{brd}, J=10.2 \mathrm{~Hz}, 1 \mathrm{H}), 5.30(\mathrm{brd}$, $J=17.4 \mathrm{~Hz}, 1 \mathrm{H}), 5.20(\mathrm{brd}, J=10.2 \mathrm{~Hz}, 1 \mathrm{H}), 5.19(\mathrm{brd}, J=17.4 \mathrm{~Hz}, 1 \mathrm{H}), 5.03(\mathrm{ddd}, J=8.4$, 5.4, 4.2 Hz, $1 \mathrm{H}), 4.87$ (dt, $J=8.4,4.2 \mathrm{~Hz}, 1 \mathrm{H}), 4.84$ (brs, $2 \mathrm{H}), 4.70(\mathrm{~d}, J=6.6 \mathrm{~Hz}, 1 \mathrm{H}), 4.69$ $(\mathrm{d}, J=6.6 \mathrm{~Hz}, 1 \mathrm{H}), 4.57(\mathrm{~d}, J=7.2 \mathrm{~Hz}, 1 \mathrm{H}), 4.52(\mathrm{~d}, J=6.6 \mathrm{~Hz}, 1 \mathrm{H}), 4.11(\mathrm{dd}, J=7.2,5.4 \mathrm{~Hz}$ $1 \mathrm{H}), 4.02$ (s, $2 \mathrm{H}), 3.99$ (q, $J=7.2 \mathrm{~Hz}, 1 \mathrm{H}), 3.39$ (s, $3 \mathrm{H}$ ), 3.36 (s, $3 \mathrm{H}$ ), 2.65 (ddq, $J$ = 9.6, 6.6, $6.6 \mathrm{~Hz}, 1 \mathrm{H}), 2.25-2.12(\mathrm{~m}, 6 \mathrm{H}), 1.76-1.48(\mathrm{~m}, 6 \mathrm{H}), 1.73(\mathrm{~s}, 3 \mathrm{H}), 1.57(\mathrm{~s}, 3 \mathrm{H}), 0.95(\mathrm{~d}, J=$ $6.6 \mathrm{~Hz}, 3 \mathrm{H}), 0.91$ (s, $9 \mathrm{H}), 0.06 \mathrm{ppm}(\mathrm{s}, 6 \mathrm{H}) ;{ }^{13} \mathrm{C} \mathrm{NMR}\left(150 \mathrm{MHz}, \mathrm{CDCl}_{3}\right): \delta=166.3,156.6$, $148.5,138.1,135.7,134.0,132.5,131.4,127.5,127.1,124.6,121.7,119.4,117.4,94.0,93.7$ $77.8,77.0,75.3,75.3,68.2,55.7,55.4,42.3,35.3,34.8,32.0,30.5,28.7,25.9,23.8,18.4,17.1$, 
16.7, 13.7, -5.2, -5.2 ppm; HRMS (ESI): calcd for $\mathrm{C}_{39} \mathrm{H}_{67} \mathrm{NO}_{9} \mathrm{SiNa}^{+}\left[\mathrm{M}+\mathrm{Na}^{+}\right] 744.4477$, found 744.4491.

Ester 51: To a solution of acid $4(110 \mathrm{mg}, 0.514 \mathrm{mmol})$ and $\mathrm{Et}_{3} \mathrm{~N}(0.18 \mathrm{~mL}, 1.25 \mathrm{mmol})$ in toluene $(1.0 \mathrm{~mL})$ was added 2,4,6-trichlorobenzoyl chloride $(80 \mu \mathrm{L}, 0.514 \mathrm{mmol})$ at room

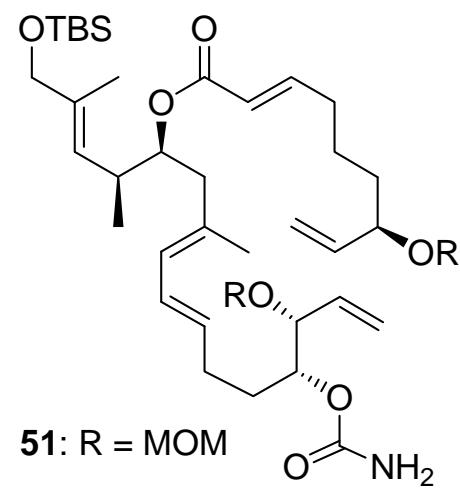
temperature. The reaction mixture was stirred for $1 \mathrm{~h}$ before a solution of alcohol 48 (135 mg, $0.257 \mathrm{mmol})$ and 4-DMAP (31 mg, $0.257 \mathrm{mmol})$ in toluene $(1.5 \mathrm{~mL})$ was added at room temperature. The reaction mixture was stirred for $12 \mathrm{~h}$ before it was quenched with $\mathrm{NH}_{4} \mathrm{Cl}$ (15 mL, sat. aq.). The resulting mixture was extracted with EtOAc $(3 \times 30 \mathrm{~mL})$, and the combined organic layers were dried $\left(\mathrm{Na}_{2} \mathrm{SO}_{4}\right)$ and concentrated in vacuo. Flash column chromatography (silica gel, hexanes:EtOAc: $\mathrm{Et}_{3} \mathrm{~N}$ 4:2:0.01) afforded ester $51(92 \mathrm{mg}, 50 \%)$ as a colorless oil. 51: $R_{\mathrm{f}}=0.35$ (silica gel, hexanes:EtOAc 3:2); $[\alpha]_{\mathrm{D}}^{25}=+2.2\left(\mathrm{CH}_{2} \mathrm{Cl}_{2}, c=0.40\right)$; IR (film) $v_{\max } 3356,2930$, 2857, 1719, 1443, 1385, 1255, $1098 \mathrm{~cm}^{-1} ;{ }^{1} \mathrm{H} \mathrm{NMR}\left(600 \mathrm{MHz}, \mathrm{CDCl}_{3}\right): \delta=6.90(\mathrm{dt}, J=15.6$, $6.9 \mathrm{~Hz}, 1 \mathrm{H}), 6.19$ (dd, $J=15.0,10.8 \mathrm{~Hz}, 1 \mathrm{H}), 5.78$ (d, $J=15.6 \mathrm{~Hz}, 1 \mathrm{H}), 5.74(\mathrm{~d}, J=10.2 \mathrm{~Hz}, 1$ H), 5.72 (ddd, $J=17.4,10.2,7.2 \mathrm{~Hz}, 1 \mathrm{H}), 5.68$ (ddd, $J=17.4,10.2,3.0 \mathrm{~Hz}, 1 \mathrm{H}), 5.50$ (dt, $J=$ 15.0, 7.2 Hz, $1 \mathrm{H}), 5.32$ (brd, $J=10.2 \mathrm{~Hz}, 1 \mathrm{H}), 5.31$ (brd, $J=17.4 \mathrm{~Hz}, 1 \mathrm{H}), 5.25$ (d, $J=10.2$ $\mathrm{Hz}, 1 \mathrm{H}), 5.21$ (brd, $J=9.6 \mathrm{~Hz}, 1 \mathrm{H}), 5.20$ (brd, $J=17.4 \mathrm{~Hz}, 1 \mathrm{H}), 4.95$ (ddd, $J=9.0,7.2,4.2 \mathrm{~Hz}$, $1 \mathrm{H}), 4.88(\mathrm{dt}, J=9.0,4.6 \mathrm{~Hz}, 1 \mathrm{H}), 4.77$ (brs, $2 \mathrm{H}), 4.71(\mathrm{~d}, J=6.6 \mathrm{~Hz}, 1 \mathrm{H}), 4.70(\mathrm{~d}, J=7.2 \mathrm{~Hz}$, $1 \mathrm{H}), 4.58(\mathrm{~d}, J=6.6 \mathrm{~Hz}, 1 \mathrm{H}), 4.53(\mathrm{~d}, J=7.2 \mathrm{~Hz}, 1 \mathrm{H}), 4.11(\mathrm{dd}, J=6.6,4.8 \mathrm{~Hz}, 1 \mathrm{H}), 4.00(\mathrm{~s}$, $2 \mathrm{H}), 3.99(\mathrm{q}, J=7.2 \mathrm{~Hz}, 1 \mathrm{H}), 3.39(\mathrm{~s}, 3 \mathrm{H}), 3.37$ (s, $3 \mathrm{H}), 2.64(\mathrm{ddq}, J=9.6,7.2,6.6 \mathrm{~Hz}, 1 \mathrm{H})$, $2.30(\mathrm{dd}, J=13.8,3.6 \mathrm{~Hz}, 1 \mathrm{H}), 2.23-2.13(\mathrm{~m}, 5 \mathrm{H}), 1.77-1.48(\mathrm{~m}, 6 \mathrm{H}), 1.72(\mathrm{~s}, 3 \mathrm{H}), 1.58(\mathrm{~s}, 3$ 
H), $0.96(\mathrm{~d}, J=6.6 \mathrm{~Hz}, 3 \mathrm{H}), 0.93(\mathrm{~s}, 9 \mathrm{H}), 0.06 \mathrm{ppm}(\mathrm{s}, 6 \mathrm{H}) ;{ }^{13} \mathrm{C} \mathrm{NMR}\left(150 \mathrm{MHz}, \mathrm{CDCl}_{3}\right): \delta=$ $166.3,156.5,148.6,138.1,135.3,134.0,132.7,131.3,127.5,127.2,125.6,121.6,119.4,117.4$ $94.0,93.7,77.8,77.0,75.7,75.4,68.2,55.7,55.4,42.5,36.0,34.8,32.0,30.4,28.7,25.9,23.8$, 18.4, 16.7, 16.6, 13.8, -5.2, $-5.2 \mathrm{ppm}$; HRMS (ESI): calcd for $\mathrm{C}_{39} \mathrm{H}_{67} \mathrm{NO}_{9} \mathrm{SiNa}^{+}\left[\mathrm{M}+\mathrm{Na}^{+}\right]$ 744.4477, found 744.4499 .

Alcohol 52: To a solution of TBS ether $49(810 \mathrm{mg}, 1.12 \mathrm{mmol})$ in THF (5 mL) was added TBAF (1.0 M in THF, $2.24 \mathrm{~mL}, 2.24 \mathrm{mmol})$ at room temperature. The reaction mixture was

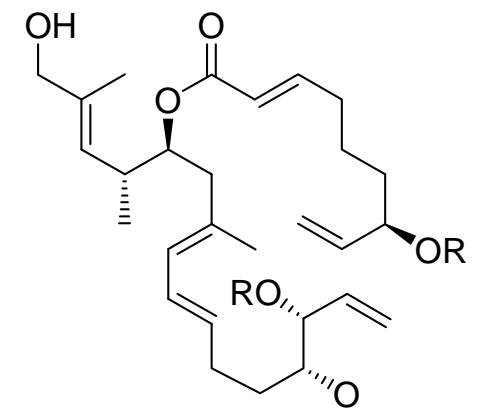
stirred for $2 \mathrm{~h}$ before it was quenched with water $(10 \mathrm{~mL})$. The resulting mixture was extracted with EtOAc $(3 \times 30 \mathrm{~mL})$, and the combined organic layers were dried $\left(\mathrm{Na}_{2} \mathrm{SO}_{4}\right)$ and concentrated in vacuo. Flash column chromatography (silica gel, hexanes:EtOAc: $\mathrm{Et}_{3} \mathrm{~N}$ 2:3:0.01) afforded alcohol 52 (593 mg, 87\%) 52: $\mathrm{R}=\mathrm{MOM}$ $\mathrm{NH}_{2}$ as a yellow oil. 52: $R_{\mathrm{f}}=0.30$ (silica gel, hexanes:EtOAc 1:2); $[\alpha]_{\mathrm{D}}^{25}=-5.1\left(\mathrm{CH}_{2} \mathrm{Cl}_{2}, c=0.9\right)$; IR (film) $v_{\max } 3450,3364,2932,2857,1714,1653,1442,1386$ $1318,1271,1149,1097,1030 \mathrm{~cm}^{-1} ;{ }^{1} \mathrm{H}$ NMR $\left(600 \mathrm{MHz}, \mathrm{CDCl}_{3}\right): \delta=6.91(\mathrm{dt}, J=15.6,7.6 \mathrm{~Hz}$ $1 \mathrm{H}), 6.20(\mathrm{dd}, J=15.0,10.8 \mathrm{~Hz}, 1 \mathrm{H}), 5.82(\mathrm{~d}, J=15.6 \mathrm{~Hz}, 1 \mathrm{H}), 5.73(\mathrm{~d}, J=10.2 \mathrm{~Hz}, 1 \mathrm{H})$, 5.71 (ddd, $J=17.4,10.2,7.2 \mathrm{~Hz}, 1 \mathrm{H}), 5.66$ (ddd, $J=17.4,10.2,7.2 \mathrm{~Hz}, 1 \mathrm{H}), 5.52$ (dt, $J=15.0$, $7.2 \mathrm{~Hz}, 1 \mathrm{H}), 5.35(\mathrm{~d}, J=10.2 \mathrm{~Hz}, 1 \mathrm{H}), 5.32(\mathrm{brd}, J=9.0 \mathrm{~Hz}, 1 \mathrm{H}), 5.31(\mathrm{brd}, J=18.6 \mathrm{~Hz}, 1 \mathrm{H})$, $5.21(\mathrm{brd}, J=8.4 \mathrm{~Hz}, 1 \mathrm{H}), 5.20(\mathrm{brd}, J=18.0 \mathrm{~Hz}, 1 \mathrm{H}), 5.03(\mathrm{ddd}, J=10.2,7.8,5.4 \mathrm{~Hz}, 1 \mathrm{H})$, $4.87(\mathrm{dt}, J=8.4,4.5 \mathrm{~Hz}, 1 \mathrm{H}), 4.81(\mathrm{brs}, 2 \mathrm{H}), 4.70(\mathrm{~d}, J=6.6 \mathrm{~Hz}, 1 \mathrm{H}), 4.69$ (d, $J=6.6 \mathrm{~Hz}, 1 \mathrm{H})$, $4.56(\mathrm{~d}, J=6.6 \mathrm{~Hz}, 1 \mathrm{H}), 4.53(\mathrm{~d}, J=6.6 \mathrm{~Hz}, 1 \mathrm{H}), 4.10(\mathrm{dd}, J=7.2,5.4 \mathrm{~Hz}, 1 \mathrm{H}), 4.00(\mathrm{~s}, 2 \mathrm{H})$, 3.99 (q, $J=7.2 \mathrm{~Hz}, 1 \mathrm{H}), 3.38$ (s, $3 \mathrm{H}), 3.37$ (s, $3 \mathrm{H}), 2.66$ (dqd, $J=11.4,7.2,5.4 \mathrm{~Hz}, 1 \mathrm{H}), 2.22$ 
(hex, $J=7.2 \mathrm{~Hz}, 4 \mathrm{H}$ ), 2.15 (pent, $J=6.6 \mathrm{~Hz}, 2 \mathrm{H}), 1.82$ (brs, $1 \mathrm{H}), 1.77-1.50$ (m, $6 \mathrm{H}), 1.74$ (s, $3 \mathrm{H}), 1.66(\mathrm{~s}, 3 \mathrm{H}), 0.97 \mathrm{ppm}(\mathrm{d}, J=6.6 \mathrm{~Hz}, 3 \mathrm{H}) ;{ }^{13} \mathrm{C} \mathrm{NMR}\left(150 \mathrm{MHz}, \mathrm{CDCl}_{3}\right): \delta=166.3$, 156.6, 148.7, 138.1, 136.1, 134.0, 132.3, 131.5, 127.6, 127.1, 126.5, 121.6, 119.4, 117.4, 94.0, $93.7,77.8,77.0,75.3,75.1,68.7,55.7,55.4,42.4,35.6,34.8,32.0,30.4,28.7,23.8,17.0,16.6$, 14.0 ppm; HRMS (ESI): calcd for $\mathrm{C}_{33} \mathrm{H}_{53} \mathrm{NO}_{9} \mathrm{Na}^{+}\left[\mathrm{M}+\mathrm{Na}^{+}\right]$630.3612, found 630.3609 .

Aldehyde 53: To a solution of alcohol $52(350 \mathrm{mg}, 0.577 \mathrm{mmol})$ in $\mathrm{CH}_{2} \mathrm{Cl}_{2}(3.0 \mathrm{~mL})$ were added $\mathrm{NaHCO}_{3}(242 \mathrm{mg}, 2.88 \mathrm{mmol})$ and DMP $(365 \mathrm{mg}, 0.865 \mathrm{mmol})$ at room temperature. The

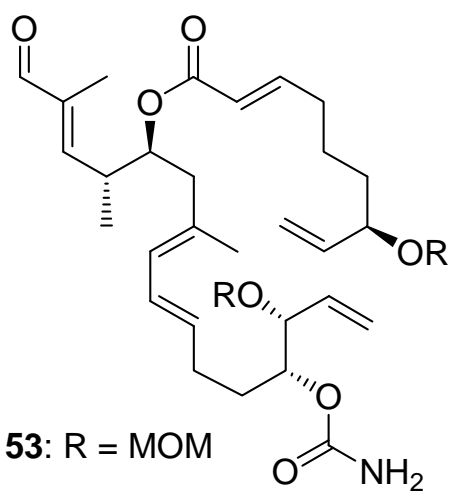
reaction mixture was stirred for $30 \mathrm{~min}$ before it was quenched with $\mathrm{NaHCO}_{3}\left(10 \mathrm{~mL}\right.$, sat. aq.) and $\mathrm{Na}_{2} \mathrm{~S}_{2} \mathrm{O}_{3}(10 \mathrm{~mL}$, sat. aq.). The resulting mixture was extracted with EtOAc $(3 \times 30 \mathrm{~mL})$, and the combined organic layers were dried $\left(\mathrm{Na}_{2} \mathrm{SO}_{4}\right)$ and concentrated in vacuo. Flash column chromatography (silica gel, hexanes:EtOAc:Et 3 N 1:1:0.01) afforded aldehyde 53 (280 mg, 80\%) as a colorless oil. 53: $R_{\mathrm{f}}=0.53$ (silica gel, hexanes:EtOAc 2:1); $[\alpha]_{\mathrm{D}}{ }^{25}=-9.2\left(\mathrm{CH}_{2} \mathrm{Cl}_{2}, c=1.05\right.$ ); IR (film) $v_{\max } 3458,3363,2933,2857,1718,1688,1651,1448,1384,1318,1275,1150,1097$, 1031, $920 \mathrm{~cm}^{-1} ;{ }^{1} \mathrm{H}$ NMR $\left(600 \mathrm{MHz}, \mathrm{CDCl}_{3}\right): \delta=9.45(\mathrm{~s}, 1 \mathrm{H}), 6.95(\mathrm{dt}, J=15.6,6.9 \mathrm{~Hz}, 1 \mathrm{H})$, $6.47(\mathrm{~d}, J=10.2 \mathrm{~Hz}, 1 \mathrm{H}), 6.20(\mathrm{dd}, J=15.0,10.8 \mathrm{~Hz}, 1 \mathrm{H}), 5.82(\mathrm{~d}, J=15.6 \mathrm{~Hz}, 1 \mathrm{H}), 5.72$ (ddd, $J=18.0,10.8,7.8 \mathrm{~Hz}, 1 \mathrm{H}), 5.71(\mathrm{~d}, J=10.8 \mathrm{~Hz}, 1 \mathrm{H}), 5.67$ (ddd, $J=17.4,10.2,7.8 \mathrm{~Hz}, 1$ H), $5.54(\mathrm{dt}, J=15.0,6.9 \mathrm{~Hz}, 1 \mathrm{H}), 5.33(\mathrm{~d}, J=8.4 \mathrm{~Hz}, 1 \mathrm{H}), 5.32(\mathrm{~d}, J=18.0 \mathrm{~Hz}, 1 \mathrm{H}), 5.23(\mathrm{~d}$, $J=7.8 \mathrm{~Hz}, 1 \mathrm{H}), 5.22(\mathrm{~d}, J=18.0 \mathrm{~Hz}, 1 \mathrm{H}), 5.16(\mathrm{q}, J=6.0 \mathrm{~Hz}, 1 \mathrm{H}), 4.88(\mathrm{dt}, J=8.4,4.8 \mathrm{~Hz}, 1$ H), 4.71 (brs, $2 \mathrm{H}), 4.71(\mathrm{~d}, J=6.6 \mathrm{~Hz}, 1 \mathrm{H}), 4.70(\mathrm{~d}, J=6.6 \mathrm{~Hz}, 1 \mathrm{H}), 4.59(\mathrm{~d}, J=6.6 \mathrm{~Hz}, 1 \mathrm{H})$, $4.54(\mathrm{~d}, J=7.2 \mathrm{~Hz}, 1 \mathrm{H}), 4.12(\mathrm{dd}, J=7.2,4.8 \mathrm{~Hz}, 1 \mathrm{H}), 4.01(\mathrm{q}, J=7.2 \mathrm{~Hz}, 1 \mathrm{H}), 3.40(\mathrm{~s}, 3 \mathrm{H})$, 
$3.38(\mathrm{~s}, 3 \mathrm{H}), 2.97$ (ddq, $J=10.8,7.2,6.6 \mathrm{~Hz}, 1 \mathrm{H}), 2.30(\mathrm{dd}, J=13.2,7.2 \mathrm{~Hz}, 1 \mathrm{H}), 2.25$ (q, $J=$ $6.6 \mathrm{~Hz}, 4 \mathrm{H}), 2.18$ (hex, $J=6.6 \mathrm{~Hz}, 2 \mathrm{H}), 1.79-1.52$ (m, $5 \mathrm{H}), 1.73$ (s, $3 \mathrm{H}), 1.65$ (s, $3 \mathrm{H}), 1.09$ $\operatorname{ppm}(\mathrm{d}, J=6.6 \mathrm{~Hz}, 3 \mathrm{H}) ;{ }^{13} \mathrm{C} \mathrm{NMR}\left(150 \mathrm{MHz}, \mathrm{CDCl}_{3}\right): \delta=195.3,166.1,156.5,154.2,149.5$, $140.0,138.1,134.0,132.2,131.2,128.2,126.9,121.1,119.4,117.4,94.0,93.7,77.8,76.9,75.3$ 74.1, 55.7, 55.5, 43.1, 36.8, 34.8, 32.1, 30.4, 28.7, 23.8, 16.6, 16.5, 9.4 ppm; HRMS (ESI): calcd for $\mathrm{C}_{33} \mathrm{H}_{51} \mathrm{NO}_{9} \mathrm{Na}^{+}\left[\mathrm{M}+\mathrm{Na}^{+}\right]$628.3456, found 628.3448.

Vinyl iodide 54: To a solution of $\mathrm{CrCl}_{2}(457 \mathrm{mg}, 3.72 \mathrm{mmol}$, flame-dried) in THF (4.5 mL) was added a solution of aldehyde $53(220 \mathrm{mg}, 0.364 \mathrm{mmol})$ and $\mathrm{CHI}_{3}(430 \mathrm{mg}, 1.10 \mathrm{mmol})$ in

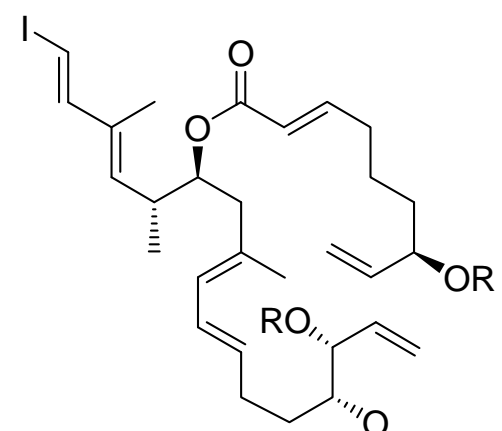

54: $\mathrm{R}=\mathrm{MOM}$ dioxane $(27 \mathrm{~mL})$ at room temperature. The reaction mixture was stirred for $2 \mathrm{~h}$ before it was quenched with $\mathrm{NaHCO}_{3}(25 \mathrm{~mL}$, sat. aq.). The resulting mixture was diluted with EtOAc $(100 \mathrm{~mL})$, filtered through a short pad of Celite ${ }^{\circledR}$ and washed with EtOAc $(100 \mathrm{~mL})$. The organic layer was separated, dried $\left(\mathrm{Na}_{2} \mathrm{SO}_{4}\right)$ and concentrated in vacuo. Flash column chromatography (silica gel, hexanes:EtOAc: $\mathrm{Et}_{3} \mathrm{~N}$ 3:2:0.01) afforded vinyl iodide $54(214 \mathrm{mg}, 81 \%)$ as a yellow oil. $54: R_{\mathrm{f}}=$ 0.65 (silica gel, hexanes:EtOAc 1:1); $[\alpha]_{\mathrm{D}}^{25}=+21.4\left(\mathrm{CH}_{2} \mathrm{Cl}_{2}, c=2.0\right.$ ); IR (film) $v_{\max } 3365,2933$, $1718,1653,1603,1442,1386,1317,1149,1097,1032,920 \mathrm{~cm}^{-1} ;{ }^{1} \mathrm{H} \mathrm{NMR}\left(600 \mathrm{MHz}, \mathrm{CDCl}_{3}\right)$ : $\delta=7.08(\mathrm{~d}, J=14.4 \mathrm{~Hz}, 1 \mathrm{H}), 6.92(\mathrm{dt}, J=15.6,6.6 \mathrm{~Hz}, 1 \mathrm{H}), 6.20(\mathrm{~d}, J=15.0 \mathrm{~Hz}, 1 \mathrm{H}), 6.18$ (dd, $J=15.0,10.2 \mathrm{~Hz}, 1 \mathrm{H}), 5.79$ (d, $J=15.6 \mathrm{~Hz}, 1 \mathrm{H}), 5.72(\mathrm{ddd}, J=17.4,10.8,7.8 \mathrm{~Hz}, 1 \mathrm{H})$, $5.71(\mathrm{~d}, J=10.8 \mathrm{~Hz}, 1 \mathrm{H}), 5.67(\mathrm{ddd}, J=18.0,10.8,7.8 \mathrm{~Hz}, 1 \mathrm{H}), 5.52(\mathrm{dt}, J=15.0,7.2 \mathrm{~Hz}, 1 \mathrm{H})$, $5.44(\mathrm{~d}, J=9.6 \mathrm{~Hz}, 1 \mathrm{H}), 5.33(\mathrm{~d}, J=9.0 \mathrm{~Hz}, 1 \mathrm{H}), 5.32(\mathrm{~d}, J=18.6 \mathrm{~Hz}, 1 \mathrm{H}), 5.22(\mathrm{~d}, J=9.0 \mathrm{~Hz}$, $1 \mathrm{H}), 5.22(\mathrm{~d}, J=18.0 \mathrm{~Hz}, 1 \mathrm{H}), 5.05(\mathrm{ddd}, J=7.8,6.0,4.2 \mathrm{~Hz}, 1 \mathrm{H}), 4.88(\mathrm{dt}, J=8.4,4.5 \mathrm{~Hz}, 1$ 
H), 4.76 (brs, $2 \mathrm{H}), 4.71(\mathrm{~d}, J=6.6 \mathrm{~Hz}, 1 \mathrm{H}), 4.70(\mathrm{~d}, J=7.2 \mathrm{~Hz}, 1 \mathrm{H}), 4.58(\mathrm{~d}, J=7.2 \mathrm{~Hz}, 1 \mathrm{H})$, $4.54(\mathrm{~d}, J=6.6 \mathrm{~Hz}, 1 \mathrm{H}), 4.11(\mathrm{dd}, J=7.2,5.4 \mathrm{~Hz}, 1 \mathrm{H}), 4.00(\mathrm{q}, J=6.6 \mathrm{~Hz}, 1 \mathrm{H}), 3.39(\mathrm{~s}, 3 \mathrm{H})$, $3.38(\mathrm{~s}, 3 \mathrm{H}), 2.72(\mathrm{dqd}, J=10.2,6.6,6.0 \mathrm{~Hz}, 1 \mathrm{H}), 2.26-2.20(\mathrm{~m}, 3 \mathrm{H}), 2.18-2.12(\mathrm{~m}, 3 \mathrm{H})$, 1.77-1.52 (m, $6 \mathrm{H}), 1.73(\mathrm{~s}, 3 \mathrm{H}), 1.72(\mathrm{~s}, 3 \mathrm{H}), 0.98 \mathrm{ppm}(\mathrm{d}, J=7.2 \mathrm{~Hz}, 3 \mathrm{H}) ;{ }^{13} \mathrm{C}$ NMR $(150$ $\left.\mathrm{MHz}, \mathrm{CDCl}_{3}\right): \delta=166.2,156.5,149.7,148.9,138.1,135.3,134.6,134.0,132.0,131.7,127.8$, $127.1,121.5,119.4,117.4,94.0,93.7,77.8,77.0,75.3,74.8,73.9,55.7,55.5,42.6,35.9,34.8$ 32.0, 30.4, 28.7, 23.8, 17.0, 16.6, 12.2 ppm; HRMS (ESI): calcd for $\mathrm{C}_{34} \mathrm{H}_{52} \mathrm{INO}_{8} \mathrm{Na}^{+}\left[\mathrm{M}+\mathrm{Na}^{+}\right]$ 752.2630 , found 752.2614 .

Diol 55 and cyclic carbonate 56: To a solution of bis-MOM ether $54(150 \mathrm{mg}, 0.206 \mathrm{mmol})$ in EtOH $(6.0 \mathrm{~mL})$ was added $\mathrm{HCl}(3.0 \mathrm{~N}$ aq., $1.5 \mathrm{~mL}, 4.5 \mathrm{mmol})$ at room temperature. The reaction

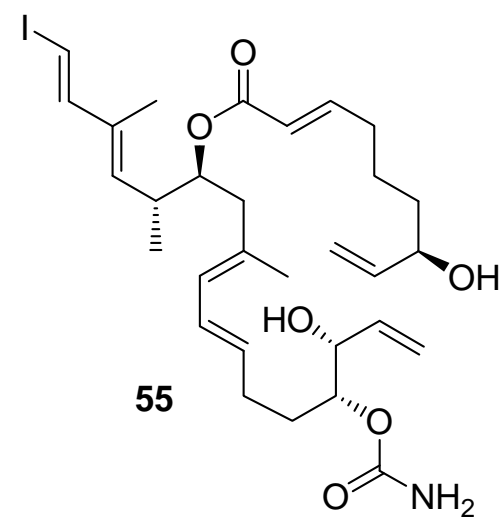
mixture was warmed to $80{ }^{\circ} \mathrm{C}$ and heated for 50 min before it was quenched with $\mathrm{NaHCO}_{3}(10 \mathrm{~mL}$, sat. aq.). The resulting mixture was extracted with EtOAc $(3 \times 30 \mathrm{~mL})$, and the combined organic layers were dried $\left(\mathrm{Na}_{2} \mathrm{SO}_{4}\right)$ and concentrated in vacuo. Flash column chromatography (silica gel, hexanes:EtOAc:Et ${ }_{3} \mathrm{~N}$ 2:5:0.01) afforded diol $55(88 \mathrm{mg}, 67 \%)$ as a yellow oil, mono-deprotected product (22 $\mathrm{mg}, 15 \%)$, and cyclic carbonate 56 (13 $\mathrm{mg}, 10 \%)$ as colorless oils. 55: $R_{\mathrm{f}}=0.21$ (silica gel, hexanes:EtOAc 1:1); $[\alpha]_{\mathrm{D}}{ }^{25}=+23.7\left(\mathrm{CH}_{2} \mathrm{Cl}_{2}, c=1.76\right.$ ); IR (film) $v_{\max } 3372,2930,1710,1651,1390,1316,1270,1180,1061,991 \mathrm{~cm}^{-1} ;{ }^{1} \mathrm{H}$ NMR $(600$ $\left.\mathrm{MHz}, \mathrm{CDCl}_{3}\right): \delta=7.09(\mathrm{~d}, J=14.4 \mathrm{~Hz}, 1 \mathrm{H}), 6.91(\mathrm{dt}, J=15.6,6.9 \mathrm{~Hz}, 1 \mathrm{H}), 6.20(\mathrm{~d}, J=14.4$ Hz, $1 \mathrm{H}), 6.18$ (dd, $J=14.4,10.8 \mathrm{~Hz}, 1 \mathrm{H}), 5.86$ (ddd, $J=17.4,11.4,6.6 \mathrm{~Hz}, 2 \mathrm{H}), 5.79$ (d, $J=$ $15.6 \mathrm{~Hz}, 1 \mathrm{H}), 5.72(\mathrm{~d}, J=10.8 \mathrm{~Hz}, 1 \mathrm{H}), 5.51(\mathrm{dt}, J=15.0,6.9 \mathrm{~Hz}, 1 \mathrm{H}), 5.44(\mathrm{~d}, J=9.6 \mathrm{~Hz}, 1$ 
H), 5.36 (brd, $J=11.4 \mathrm{~Hz}, 1 \mathrm{H}), 5.25$ (brd, $J=16.8 \mathrm{~Hz}, 1 \mathrm{H}), 5.24$ (d, $J=12.0 \mathrm{~Hz}, 1 \mathrm{H}), 5.13$ (brd, $J=10.2 \mathrm{~Hz}, 1 \mathrm{H}), 5.05$ (dt, $J=7.2,5.4 \mathrm{~Hz}, 1 \mathrm{H}), 4.80$ (brs, $2 \mathrm{H}), 4.74$ (dt, $J=8.4,4.8 \mathrm{~Hz}, 1$ H), 4.17-4.10 (m, $2 \mathrm{H}), 2.73$ (ddq, $J=10.8,6.6,6.6 \mathrm{~Hz}, 1 \mathrm{H}), 2.27-2.20(\mathrm{~m}, 3 \mathrm{H}), 2.19-2.13(\mathrm{~m}$, $3 \mathrm{H}), 1.78-1.52(\mathrm{~m}, 6 \mathrm{H}), 1.71(\mathrm{~s}, 3 \mathrm{H}), 1.70(\mathrm{~s}, 3 \mathrm{H}), 0.96 \mathrm{ppm}(\mathrm{d}, J=7.2 \mathrm{~Hz}, 3 \mathrm{H}) ;{ }^{13} \mathrm{C} \mathrm{NMR}$ $\left(150 \mathrm{MHz}, \mathrm{CDCl}_{3}\right): \delta=166.2,157.0,149.6,148.9,141.0,136.9,135.2,134.7,132.0,131.6$ $127.8,127.2,121.5,117.0,114.8,76.9,74.6,74.2,74.0,72.9,42.7,36.4,36.1,32.0,30.4,28.7$, 23.8, 17.0, 16.7, 12.2 ppm; HRMS (ESI): calcd for $\mathrm{C}_{30} \mathrm{H}_{44} \mathrm{INO}_{6} \mathrm{Na}^{+}\left[\mathrm{M}+\mathrm{Na}^{+}\right]$664.2106, found 664.2083.

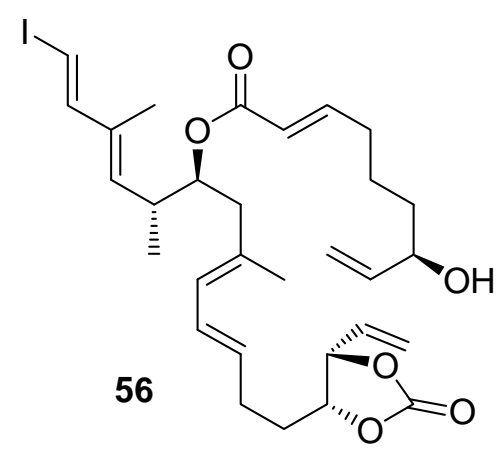

Cyclic carbonate 56: $R_{\mathrm{f}}=0.54$ (silica gel, hexanes:EtOAc 2:1); $[\alpha]_{\mathrm{D}}^{25}=+31.9\left(\mathrm{CH}_{2} \mathrm{Cl}_{2}, c=0.60\right) ;$ IR (film) $v_{\max } 3471,2928$, $2859,1805,1713,1652,1433,1367,1267,1187,1049,989,951$ $\mathrm{cm}^{-1} ;{ }^{1} \mathrm{H}$ NMR $\left(600 \mathrm{MHz}, \mathrm{CDCl}_{3}\right): \delta=7.09(\mathrm{~d}, J=15.0 \mathrm{~Hz}, 1 \mathrm{H})$, $6.92(\mathrm{dt}, J=15.6,6.9 \mathrm{~Hz}, 1 \mathrm{H}), 6.23(\mathrm{dd}, J=15.0,10.8 \mathrm{~Hz}, 1 \mathrm{H})$, $6.21(\mathrm{~d}, J=15.0 \mathrm{~Hz}, 1 \mathrm{H}), 5.87$ (ddd, $J=17.4,10.8,7.8 \mathrm{~Hz}, 2 \mathrm{H}), 5.80(\mathrm{~d}, J=15.6 \mathrm{~Hz}, 1 \mathrm{H})$, $5.72(\mathrm{~d}, J=10.8 \mathrm{~Hz}, 1 \mathrm{H}), 5.50$ (d, $J=16.8 \mathrm{~Hz}, 1 \mathrm{H}), 5.46(\mathrm{dt}, J=15.0,7.2 \mathrm{~Hz}, 1 \mathrm{H}), 5.44$ (d, $J$ $=10.2 \mathrm{~Hz}, 2 \mathrm{H}), 5.24(\mathrm{~d}, J=17.4 \mathrm{~Hz}, 1 \mathrm{H}), 5.13(\mathrm{~d}, J=10.2 \mathrm{~Hz}, 1 \mathrm{H}), 5.06(\mathrm{ddd}, J=8.4,5.4,4.2$ $\mathrm{Hz}, 1 \mathrm{H}), 4.66(\mathrm{t}, J=7.2 \mathrm{~Hz}, 1 \mathrm{H}), 4.32(\mathrm{ddd}, J=11.4,7.5,4.2 \mathrm{~Hz}, 1 \mathrm{H}), 4.16-4.10(\mathrm{~m}, 1 \mathrm{H})$, $2.73(\mathrm{dqd}, J=10.8,7.2,6.6 \mathrm{~Hz}, 1 \mathrm{H}), 2.33-2.16(\mathrm{~m}, 6 \mathrm{H}), 1.92-1.85(\mathrm{~m}, 1 \mathrm{H}), 1.82-1.71(\mathrm{~m}, 2$ H), $1.75(\mathrm{~s}, 3 \mathrm{H}), 1.73(\mathrm{~s}, 3 \mathrm{H}), 1.64-1.51(\mathrm{~m}, 3 \mathrm{H}), 0.99 \mathrm{ppm}(\mathrm{d}, J=7.2 \mathrm{~Hz}, 3 \mathrm{H}) ;{ }^{13} \mathrm{C}$ NMR $\left(150 \mathrm{MHz}, \mathrm{CDCl}_{3}\right): \delta=166.2,154.2,149.6,148.9,141.0,135.3,134.6,133.2,132.0,129.5$ $128.3,127.2,121.5,121.4,114.9,82.6,81.1,74.7,74.0,72.9,42.6,36.3,36.0,32.8,32.0,28.0$, 23.8, 17.0, 16.7, $12.2 \mathrm{ppm}$; HRMS (ESI): calcd for $\mathrm{C}_{30} \mathrm{H}_{41} \mathrm{IO}_{6} \mathrm{Na}^{+}\left[\mathrm{M}+\mathrm{Na}^{+}\right]$647.1840, found 647.1847. 
Macrocycle 57: To a solution of diol $55(64 \mathrm{mg}, 0.10 \mathrm{mmol})$ in $\mathrm{CH}_{2} \mathrm{Cl}_{2}(50 \mathrm{~mL})$ was added Grubbs II catalyst $(17 \mathrm{mg}, 0.02 \mathrm{mmol})$ at room temperature. The reaction mixture was stirred for

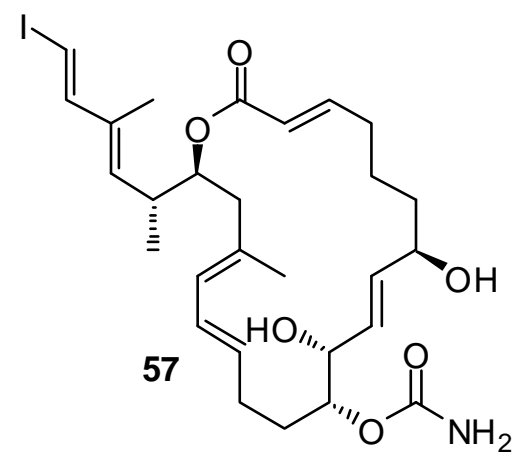
$2 \mathrm{~h}$ before it was concentrated in vacuo. Flash column chromatography (silica gel, MeOH:EtOAc:Et ${ }_{3} \mathrm{~N}$ 2:100:1) afforded macrocycle $57(38 \mathrm{mg}, 62 \%)$ as a colorless oil. $57: R_{\mathrm{f}}=$ 0.40 (silica gel, EtOAc); $[\alpha]_{\mathrm{D}}^{25}=+44.8\left(\mathrm{CH}_{2} \mathrm{Cl}_{2}, c=0.50\right)$; IR (film) $v_{\max } 3359,2926,2856,1714,1655,1447,1391,1315$,

1261, 1186, 1077, $975 \mathrm{~cm}^{-1} ;{ }^{1} \mathrm{H}$ NMR (600 MHz, $\left.\mathrm{CDCl}_{3}\right): \delta=7.07(\mathrm{~d}, J=15.0 \mathrm{~Hz}, 1 \mathrm{H}), 6.82$ (ddd, $J=15.6,10.2,5.4 \mathrm{~Hz}, 1 \mathrm{H}), 6.21(\mathrm{~d}, J=14.4 \mathrm{~Hz}, 1 \mathrm{H}), 6.14(\mathrm{dd}, J=14.7,10.5 \mathrm{~Hz}, 1 \mathrm{H})$, $5.76(\mathrm{dd}, J=15.6,3.0 \mathrm{~Hz}, 1 \mathrm{H}), 5.73(\mathrm{~d}, J=14.4 \mathrm{~Hz}, 1 \mathrm{H}), 5.68(\mathrm{dd}, J=15.6,8.4 \mathrm{~Hz}, 1 \mathrm{H}), 5.66$ $(\mathrm{d}, J=10.2 \mathrm{~Hz}, 1 \mathrm{H}), 5.41(\mathrm{ddd}, J=14.4,9.6,4.8 \mathrm{~Hz}, 1 \mathrm{H}), 5.40(\mathrm{~d}, J=9.6 \mathrm{~Hz}, 1 \mathrm{H}), 5.11(\mathrm{dt}, J$ $=7.8,4.6 \mathrm{~Hz}, 1 \mathrm{H}), 4.85$ (brs, $2 \mathrm{H}), 4.74(\mathrm{td}, J=8.4,4.2 \mathrm{~Hz}, 1 \mathrm{H}), 4.34$ (brs, $1 \mathrm{H}), 4.05(\mathrm{t}, J=8.1$ Hz, $1 \mathrm{H}), 3.01$ (brs, $1 \mathrm{H}), 2.72$ (ddq, $J=11.4,7.2,6.6 \mathrm{~Hz}, 1 \mathrm{H}), 2.25-2.08$ (m, $4 \mathrm{H}), 2.06-1.95$ (m, $2 \mathrm{H}), 1.75(\mathrm{~s}, 3 \mathrm{H}), 1.72(\mathrm{~s}, 3 \mathrm{H}), 1.66-1.57(\mathrm{~m}, 1 \mathrm{H}), 1.52-1.45(\mathrm{~m}, 4 \mathrm{H}), 1.37-1.28(\mathrm{~m}, 1$ H), $1.02 \mathrm{ppm}(\mathrm{d}, J=6.6 \mathrm{~Hz}, 3 \mathrm{H}) ;{ }^{13} \mathrm{C} \mathrm{NMR}\left(150 \mathrm{MHz}, \mathrm{CDCl}_{3}\right): \delta=166.3,157.1,149.6,148.7$, $135.1,135.0,134.3,131.7,131.3,129.5,128.1,127.2,121.4,77.5,74.0,73.5,72.9,72.2,43.4$, 37.0, 36.8, 32.7, 30.3, 28.9, 24.6, 17.0, 16.5, 12.2 ppm; HRMS (ESI): calcd for $\mathrm{C}_{28} \mathrm{H}_{40} \mathrm{INO}_{6} \mathrm{Na}^{+}$ $\left[\mathrm{M}+\mathrm{Na}^{+}\right]$636.1792, found 636.1780 .

Palmerolide A isomer 19-epi-1 and decarbamated palmerolide A isomer 58: To a solution of vinyl iodide $57(7.0 \mathrm{mg}, 0.0114 \mathrm{mmol})$, amide 42 (2.2 mg, $0.023 \mathrm{mmol})$, CuI (2.2 mg, 0.0116 mmol) and $\mathrm{K}_{2} \mathrm{CO}_{3}\left(8.0 \mathrm{mg}, 0.058 \mathrm{mmol}\right.$, flame dried) in DMF (1.0 mL) was added $N, N^{\prime}-$ dimethylethylenediamine $(4.0 \mu \mathrm{L}, 0.036 \mathrm{mmol})$ at room temperature. The reaction mixture was 


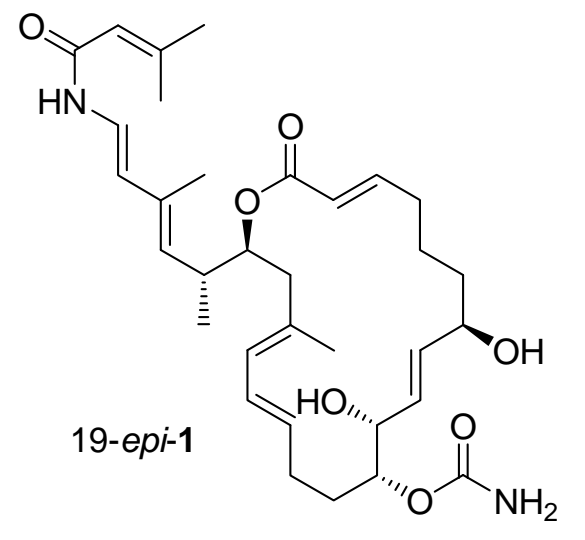

stirred for $1 \mathrm{~h}$ before it was diluted with EtOAc $(20 \mathrm{~mL})$, filtered through a short pad of Celite ${ }^{\circledR}$ and concentrated in vacuo. Preparative TLC purification (silica gel, hexanes:EtOAc 0:1) afforded 19-epi-1 (2.5 $\mathrm{mg}, 37 \%)$ as a colorless oil and decarbamated product $58(0.6 \mathrm{mg}, 10 \%)$ as a colorless oil. Palmerolide A isomer 19-epi-1: $R_{\mathrm{f}}=0.25$ (silica gel, hexanes:EtOAc 0:1); $[\alpha]_{D}^{25}=-13.8(\mathrm{MeOH}, c=0.21)$; IR (film) $v_{\max } 3356,2926,2855$, $1714,1640,1520,1391,1191 \mathrm{~cm}^{-1} ;{ }^{1} \mathrm{H}$ NMR $\left(600 \mathrm{MHz}, \mathrm{DMSO}-d^{6}\right): \delta=9.88(\mathrm{~d}, J=10.2 \mathrm{~Hz}, 1$ H), 6.82 (dd, $J=15.0,10.2 \mathrm{~Hz}, 1 \mathrm{H}), 6.68$ (ddd, $J=15.0,9.6,4.8 \mathrm{~Hz}, 1 \mathrm{H}), 6.49$ (brs, $2 \mathrm{H}$ ), 6.04 (dd, $J=14.7,10.5 \mathrm{~Hz}, 1 \mathrm{H}), 5.83(\mathrm{~d}, J=14.4 \mathrm{~Hz}, 1 \mathrm{H}), 5.73(\mathrm{~d}, J=16.2 \mathrm{~Hz}, 1 \mathrm{H}), 5.68(\mathrm{~s}, 1 \mathrm{H})$, $5.57(\mathrm{~d}, J=10.2 \mathrm{~Hz}, 1 \mathrm{H}), 5.54(\mathrm{dd}, J=15.6,7.8 \mathrm{~Hz}, 1 \mathrm{H}), 5.47$ (dd, $J=15.6,3.0 \mathrm{~Hz}, 1 \mathrm{H}), 5.40$ (ddd, $J=15.0,10.2,4.8 \mathrm{~Hz}, 1 \mathrm{H}), 5.21(\mathrm{~d}, J=4.8 \mathrm{~Hz}, 1 \mathrm{H}), 5.13$ (d, $J=9.6 \mathrm{~Hz}, 1 \mathrm{H}), 4.93$ (ddd, $J=11.4,4.8,1.8 \mathrm{~Hz}, 1 \mathrm{H}), 4.76(\mathrm{~d}, J=4.2 \mathrm{~Hz}, 1 \mathrm{H}), 4.47(\mathrm{ddd}, J=10.8,6.6,1.8 \mathrm{~Hz}, 1 \mathrm{H}), 4.12$ (m, $1 \mathrm{H}), 3.82(\mathrm{~m}, 1 \mathrm{H}), 2.72(\mathrm{ddq}, J=13.8,7.2,6.6 \mathrm{~Hz}, 1 \mathrm{H}), 2.17-1.85(\mathrm{~m}, 6 \mathrm{H}), 2.10(\mathrm{~s}, 3 \mathrm{H})$, $1.82(\mathrm{~s}, 3 \mathrm{H}), 1.68$ (s, $3 \mathrm{H}), 1.62$ (s, $3 \mathrm{H}), 1.59-1.54(\mathrm{~m}, 1 \mathrm{H}), 1.49-1.45(\mathrm{~m}, 1 \mathrm{H}), 1.30-1.24$ (m, $2 \mathrm{H}), 1.06-0.95(\mathrm{~m}, 2 \mathrm{H}), 0.92 \mathrm{ppm}(\mathrm{d}, J=6.6 \mathrm{~Hz}, 3 \mathrm{H}) ;{ }^{13} \mathrm{C}$ NMR $\left(150 \mathrm{MHz}\right.$, DMSO- $\left.d^{6}\right): \delta=$ $165.8,163.6,157.1,152.2,149.5,134.1,133.1,132.4,131.9,130.0,129.3,128.1,126.8,122.4$, $121.2,118.6,117.0,75.6,74.0,72.9,69.7,43.4,38.2,36.8,32.8,29.9,29.8,27.5,25.4,20.0$, 17.9, 16.7, 13.0 ppm; HRMS (ESI): calcd for $\mathrm{C}_{33} \mathrm{H}_{48} \mathrm{~N}_{2} \mathrm{O}_{7} \mathrm{Na}^{+}\left[\mathrm{M}+\mathrm{Na}^{+}\right]$607.3354, found 607.3342 .

Decarbamated palmerolide A isomer 58: $R_{\mathrm{f}}=0.28$ (silica gel, hexanes:EtOAc 0:1); $[\alpha]_{\mathrm{D}}^{25}=-3.9$ $(\mathrm{MeOH}, c=0.18)$; IR (film) $v_{\max } 3372,2927,1714,1640,1522,1392,1315,1276,1261,1194$ 1154, 1077, 976, $764 \mathrm{~cm}^{-1} ;{ }^{1} \mathrm{H}$ NMR $\left(600 \mathrm{MHz}, \mathrm{DMSO}-d^{6}\right): \delta=9.81(\mathrm{~d}, J=10.2 \mathrm{~Hz}, 1 \mathrm{H}), 6.84$ 


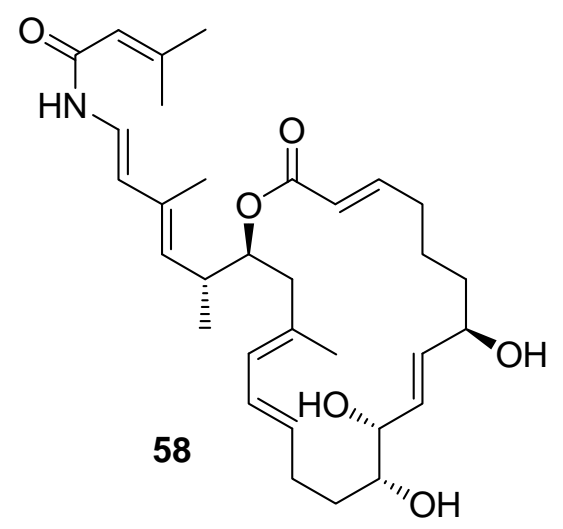

(dd, $J=14.4,10.2 \mathrm{~Hz}, 1 \mathrm{H}), 6.70(\mathrm{ddd}, J=15.6,9.6,5.4 \mathrm{~Hz}, 1$ H), 6.05 (dd, $J=15.0,10.8 \mathrm{~Hz}, 1 \mathrm{H}), 5.83(\mathrm{~d}, J=14.4 \mathrm{~Hz}, 1$ H), $5.74(\mathrm{~d}, J=15.6 \mathrm{~Hz}, 1 \mathrm{H}), 5.69$ (brs, $1 \mathrm{H}), 5.59$ (d, $J=9.6$ $\mathrm{Hz}, 1 \mathrm{H}), 5.57$ (dd, $J=15.6,3.6 \mathrm{~Hz}, 1 \mathrm{H}), 5.49$ (dd, $J=15.6$, $7.8 \mathrm{~Hz}, 1 \mathrm{H}), 5.43$ (ddd, $J=15.0,10.8,4.8 \mathrm{~Hz}, 1 \mathrm{H}), 5.14$ (d, $J$ $=9.6 \mathrm{~Hz}, 1 \mathrm{H}), 4.95(\mathrm{ddd}, J=11.4,4.8,1.8 \mathrm{~Hz}, 1 \mathrm{H}), 4.76(\mathrm{~d}$, $J=4.8 \mathrm{~Hz}, 1 \mathrm{H}), 4.64(\mathrm{~d}, J=4.2 \mathrm{~Hz}, 1 \mathrm{H}), 4.58(\mathrm{~d}, J=4.2 \mathrm{~Hz}, 1 \mathrm{H}), 3.95(\mathrm{~m}, 1 \mathrm{H}), 3.82$ (ddd, $J$ $=12.6,8.4,5.4 \mathrm{~Hz}, 1 \mathrm{H}), 3.33(\mathrm{~m}, 1 \mathrm{H}), 2.74(\mathrm{dqd}, J=9.6,6.6,4.8 \mathrm{~Hz}, 1 \mathrm{H}), 2.18-2.01(\mathrm{~m}, 6 \mathrm{H})$, 2.12 (s, $3 \mathrm{H}), 1.93-1.86(\mathrm{~m}, 1 \mathrm{H}), 1.83(\mathrm{~s}, 3 \mathrm{H}), 1.70(\mathrm{~s}, 3 \mathrm{H}), 1.63$ (s, $3 \mathrm{H}), 1.48-1.38$ (m, $2 \mathrm{H})$, $1.32-1.22(\mathrm{~m}, 2 \mathrm{H}), 1.10-1.03(\mathrm{~m}, 1 \mathrm{H}), 0.94 \mathrm{ppm}(\mathrm{d}, J=6.6 \mathrm{~Hz}, 3 \mathrm{H}) ;{ }^{13} \mathrm{C}$ NMR $(150 \mathrm{MHz}$, DMSO- $\left.d^{6}\right): \delta=165.7,163.6,152.1,149.5,133.3,133.2,133.1,131.4,130.2,130.1,128.3$ $126.5,122.4,121.2,118.6,117.0,74.0,73.5,72.6,43.2,38.2,36.8,32.8,32.6,30.0,27.4,25.1$, 20.0, 17.8, 16.7, 13.0 ppm; HRMS (ESI): calcd for $\mathrm{C}_{32} \mathrm{H}_{47} \mathrm{NO}_{6} \mathrm{Na}^{+}\left[\mathrm{M}+\mathrm{Na}^{+}\right] 564.3296$, found 564.3295 .

Triol 59: To a solution of cyclic carbonate $56(30 \mathrm{mg}, 0.048 \mathrm{mmol})$ in $\mathrm{MeOH}(1.2 \mathrm{~mL})$ was added $\mathrm{NaOMe}(40 \mathrm{mg}, 0.74 \mathrm{mmol})$ at room temperature. The reaction mixture was stirred for $2 \mathrm{~h}$

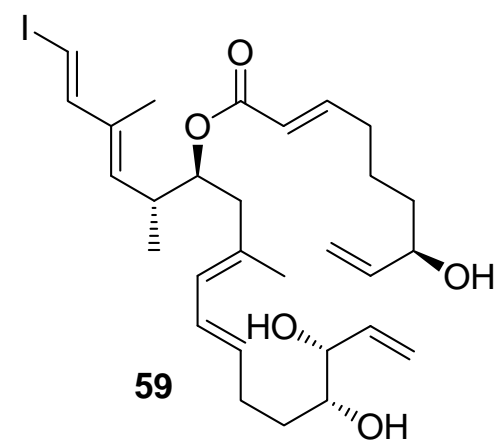
before it was quenched with $\mathrm{NH}_{4} \mathrm{Cl}(20 \mathrm{~mL}$, sat. aq.). The resulting mixture was extracted with EtOAc $(3 \times 30 \mathrm{~mL})$, and the combined organic layers were dried $\left(\mathrm{Na}_{2} \mathrm{SO}_{4}\right)$ and concentrated in vacuo. Flash column chromatography (silica gel, hexanes:EtOAc:Et $3 \mathrm{~N}$ 2:5:0.01) afforded triol 59 (16 mg, 56\%) as a colorless oil. 59: $R_{\mathrm{f}}=0.30$ (silica gel, hexanes:EtOAc 1:1); $[\alpha]_{\mathrm{D}}{ }^{25}=+23.5\left(\mathrm{CH}_{2} \mathrm{Cl}_{2}, c=0.55\right.$ ); 
IR (film) $v_{\max } 3414,2926,1714,1653,1438,1389,1275,1180,991 \mathrm{~cm}^{-1} ;{ }^{1} \mathrm{H}$ NMR $(600 \mathrm{MHz}$, $\left.\mathrm{CDCl}_{3}\right): \delta=7.09(\mathrm{~d}, J=14.4 \mathrm{~Hz}, 1 \mathrm{H}), 6.92(\mathrm{dt}, J=15.6,6.9 \mathrm{~Hz}, 1 \mathrm{H}), 6.22(\mathrm{dd}, J=14.4,10.8$ $\mathrm{Hz}, 1 \mathrm{H}), 6.21$ (d, $J=14.4 \mathrm{~Hz}, 1 \mathrm{H}), 5.86$ (ddd, $J=17.4,11.4,6.6 \mathrm{~Hz}, 2 \mathrm{H}), 5.80$ (d, $J=15.0 \mathrm{~Hz}$, $1 \mathrm{H}), 5.72(\mathrm{~d}, J=10.2 \mathrm{~Hz}, 1 \mathrm{H}), 5.55(\mathrm{dt}, J=15.0,7.2 \mathrm{~Hz}, 1 \mathrm{H}), 5.44(\mathrm{~d}, J=9.6 \mathrm{~Hz}, 1 \mathrm{H}), 5.37$ (brd, $J=17.4 \mathrm{~Hz}, 1 \mathrm{H}), 5.26(\mathrm{~d}, J=10.8 \mathrm{~Hz}, 1 \mathrm{H}), 5.24$ (brd, $J=17.4 \mathrm{~Hz}, 1 \mathrm{H}), 5.14$ (brd, $J=$ $10.2 \mathrm{~Hz}, 1 \mathrm{H}), 5.06$ (ddd, $J=8.4,5.4,4.2 \mathrm{~Hz}, 1 \mathrm{H}), 4.16-4.10(\mathrm{~m}, 1 \mathrm{H}), 3.95(\mathrm{t}, J=6.0 \mathrm{~Hz}, 1 \mathrm{H})$, $3.50(\operatorname{td}, J=6.0,3.6 \mathrm{~Hz}, 1 \mathrm{H}), 3.49(\mathrm{~d}, J=2.4 \mathrm{~Hz}, 1 \mathrm{H}), 3.32(\mathrm{~d}, J=3.6 \mathrm{~Hz}, 1 \mathrm{H}), 2.73(\mathrm{dqd}, J=$ 10.8, 7.2, 6.6 Hz, $1 \mathrm{H}), 2.32-2.17$ (m, $6 \mathrm{H}), 1.64-1.50$ (m, $6 \mathrm{H}), 1.74(\mathrm{~s}, 3 \mathrm{H}), 1.73$ (s, $3 \mathrm{H}), 1.00$ $\operatorname{ppm}(\mathrm{d}, J=7.2 \mathrm{~Hz}, 3 \mathrm{H}) ;{ }^{13} \mathrm{C} \mathrm{NMR}\left(150 \mathrm{MHz}, \mathrm{CDCl}_{3}\right): \delta=166.2,149.7,148.9,141.0,137.6$, $135.2,134.7,132.2,131.9,127.8,127.1,121.5,117.5,114.9,76.1,74.6,73.9,73.8,72.9,42.7$, 36.3, 36.0, 32.6, 32.0, 29.0, 23.8, 17.0, 16.7, $12.2 \mathrm{ppm}$; HRMS (ESI): calcd for $\mathrm{C}_{29} \mathrm{H}_{43} \mathrm{IO}_{5} \mathrm{Na}^{+}[\mathrm{M}$ $\left.+\mathrm{Na}^{+}\right]$621.2047, found 621.2045.

Macrocycle 60: To a solution of triol $59(16 \mathrm{mg}, 0.0268 \mathrm{mmol})$ in $\mathrm{CH}_{2} \mathrm{Cl}_{2}(14 \mathrm{~mL})$ was added Grubbs II catalyst $(4.5 \mathrm{mg}, 0.0054 \mathrm{mmol})$ at room temperature. The reaction mixture was stirred

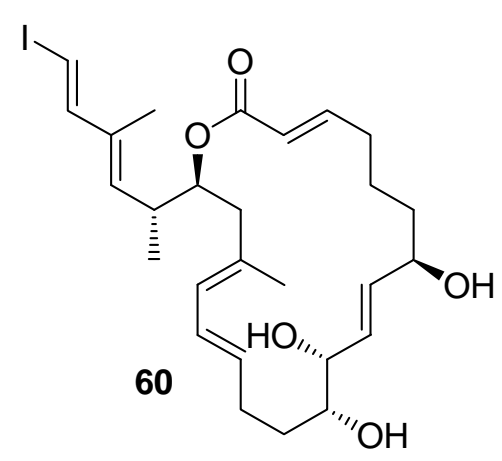
for $2 \mathrm{~h}$ before it was concentrated in vacuo. Preparative TLC purification (silica gel, hexanes:EtOAc 0:1) afforded macrocycle 60 $(10 \mathrm{mg}, 65 \%)$ as a colorless oil. 60: $R_{\mathrm{f}}=0.46$ (silica gel, EtOAc); $[\alpha]_{\mathrm{D}}^{25}=+51.2\left(\mathrm{CH}_{2} \mathrm{Cl}_{2}, c=0.45\right)$; IR (film) $v_{\max } 3374$, $2928,2858,1716,1655,1445,1388,1275,1261,1186,1078,975$, $751 \mathrm{~cm}^{-1} ;{ }^{1} \mathrm{H}$ NMR $\left(600 \mathrm{MHz}, \mathrm{CDCl}_{3}\right): \delta=7.07(\mathrm{~d}, J=14.4 \mathrm{~Hz}, 1 \mathrm{H}), 6.84(\mathrm{ddd}, J=15.6,8.4$, $5.4 \mathrm{~Hz}, 1 \mathrm{H}), 6.21(\mathrm{~d}, J=14.4 \mathrm{~Hz}, 1 \mathrm{H}), 6.18(\mathrm{dd}, J=15.0,10.2 \mathrm{~Hz}, 1 \mathrm{H}), 5.79(\mathrm{dd}, J=15.6,6.6$ $\mathrm{Hz}, 1 \mathrm{H}), 5.74(\mathrm{~d}, J=15.0 \mathrm{~Hz}, 1 \mathrm{H}), 5.69(\mathrm{dd}, J=15.6,5.4 \mathrm{~Hz}, 1 \mathrm{H}), 5.68(\mathrm{~d}, J=10.2 \mathrm{~Hz}, 1 \mathrm{H})$, 
$5.54(\mathrm{ddd}, J=15.0,9.6,5.4 \mathrm{~Hz}, 1 \mathrm{H}), 5.39$ (d, $J=9.6 \mathrm{~Hz}, 1 \mathrm{H}), 5.11$ (ddd, $J=7.8,4.8,3.0 \mathrm{~Hz}, 1$ H), $4.15(\mathrm{q}, J=6.0 \mathrm{~Hz}, 1 \mathrm{H}), 3.99$ (t, $J=6.6 \mathrm{~Hz}, 1 \mathrm{H}), 3.52-3.47$ (m, $1 \mathrm{H}), 2.73$ (ddq, $J=11.4$, 7.2, 6.6 Hz, $1 \mathrm{H}), 2.32-2.12$ (m, $6 \mathrm{H}), 2.06-1.95$ (m, $2 \mathrm{H}), 1.75$ (s, $3 \mathrm{H}), 1.72$ (s, $3 \mathrm{H}), 1.69-1.45$ (m, $4 \mathrm{H}), 1.35-1.27(\mathrm{~m}, 2 \mathrm{H}), 1.02 \mathrm{ppm}(\mathrm{d}, J=6.6 \mathrm{~Hz}, 3 \mathrm{H}) ;{ }^{13} \mathrm{C}$ NMR $\left(150 \mathrm{MHz}, \mathrm{CDCl}_{3}\right): \delta=$ $166.2,149.6,148.6,135.2,135.0,134.7,131.8,131.4,129.8,127.8,127.4,121.5,75.5,74.0$ 74.0, 73.4, 72.2, 43.1, 37.0, 36.5, 32.8, 32.4, 28.4, 24.2, 16.8, 16.5, 12.2 ppm; HRMS (ESI): calcd for $\mathrm{C}_{27} \mathrm{H}_{39} \mathrm{IO}_{5} \mathrm{Na}^{+}\left[\mathrm{M}+\mathrm{Na}^{+}\right]$593.1734, found 593.1730.

Enamide 58: To a mixture of vinyl iodide 60 (10.0 mg, $0.0176 \mathrm{mmol})$, amide 42 (3.5 mg, 0.035 mmol), $\mathrm{CuI}(3.3 \mathrm{mg}, 0.0176 \mathrm{mmol})$ and $\mathrm{K}_{2} \mathrm{CO}_{3}(12.0 \mathrm{mg}, 0.087 \mathrm{mmol}$, flame dried) in DMF (1.7

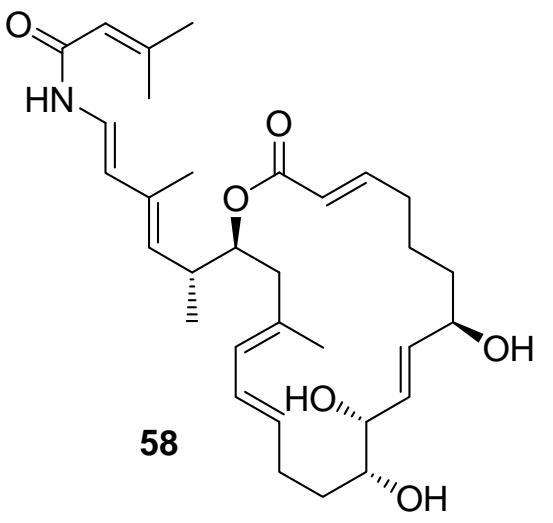

$\mathrm{mL})$ was added $N, N^{\prime}$-dimethylethylenediamine $(5.8 \mu \mathrm{L}, 0.053$ $\mathrm{mmol})$ at room temperature. The reaction mixture was stirred for $1 \mathrm{~h}$ before it was diluted with EtOAc $(20 \mathrm{~mL})$, filtered through a short pad of Celite ${ }^{\circledR}$ and concentrated in vacuo. Preparative TLC purification (silica gel, hexanes:EtOAc 1:8) afforded enamide $58(4.3 \mathrm{mg}, 45 \%)$ as a colorless oil. All physical properties of this compound were identical to those reported for decarbamted product 58 obtained from vinyl iodide 57.

Alcohol 61: To a solution of TBS ether $50(780 \mathrm{mg}, 1.08 \mathrm{mmol})$ in THF (5 mL) was added TBAF (1.0 M in THF, $2.0 \mathrm{~mL}, 2.0 \mathrm{mmol})$ at room temperature. The reaction mixture was stirred for $2 \mathrm{~h}$ before it was quenched with water $(10 \mathrm{~mL})$. The resulting mixture was extracted with EtOAc $(3 \times 30 \mathrm{~mL})$, and the combined organic layers were dried $\left(\mathrm{Na}_{2} \mathrm{SO}_{4}\right)$ and concentrated in 


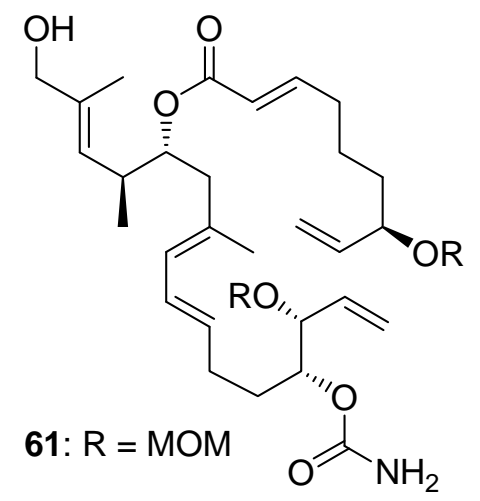

vacuo. Flash column chromatography (silica gel, hexanes:EtOAc:Et ${ }_{3} \mathrm{~N}$ 2:3:0.01) afforded alcohol $61(560 \mathrm{mg}, 85 \%)$ as a yellow oil. 61: $R_{\mathrm{f}}=0.30$ (silica gel, hexanes:EtOAc 1:2); $[\alpha]_{\mathrm{D}}{ }^{25}$ $=+19.9\left(\mathrm{CH}_{2} \mathrm{Cl}_{2}, c=0.88\right)$; IR (film) $v_{\max } 3451,3363,2932,1714$, $1653,1442,1386,1318,1271,1149,1097,1031 \mathrm{~cm}^{-1} ;{ }^{1} \mathrm{H}$ NMR $\left(600 \mathrm{MHz}, \mathrm{CDCl}_{3}\right): \delta=6.92(\mathrm{dt}, J=15.6,6.9 \mathrm{~Hz}, 1 \mathrm{H}), 6.21(\mathrm{dd}, J$ $=15.0,10.8 \mathrm{~Hz}, 1 \mathrm{H}), 5.80(\mathrm{~d}, J=15.6 \mathrm{~Hz}, 1 \mathrm{H}), 5.73(\mathrm{~d}, J=10.2 \mathrm{~Hz}, 1 \mathrm{H}), 5.72(\mathrm{ddd}, J=16.2$, 9.6, 6.6 Hz, $1 \mathrm{H}), 5.67$ (ddd, $J=18.0,10.2,7.8 \mathrm{~Hz}, 1 \mathrm{H}), 5.55(\mathrm{dt}, J=15.0,6.9 \mathrm{~Hz}, 1 \mathrm{H}), 5.36(\mathrm{~d}$, $J=9.6 \mathrm{~Hz}, 1 \mathrm{H}), 5.33(\mathrm{~d}, J=9.0 \mathrm{~Hz}, 1 \mathrm{H}), 5.32(\mathrm{~d}, J=19.2 \mathrm{~Hz}, 1 \mathrm{H}), 5.22(\mathrm{~d}, J=9.0 \mathrm{~Hz}, 1 \mathrm{H})$, $5.21(\mathrm{~d}, J=18.6 \mathrm{~Hz}, 1 \mathrm{H}), 5.03(\mathrm{dt}, J=6.6,4.8 \mathrm{~Hz}, 1 \mathrm{H}), 4.89(\mathrm{dt}, J=7.8,4.8 \mathrm{~Hz}, 1 \mathrm{H}), 4.72$ (brs, $2 \mathrm{H}), 4.71(\mathrm{~d}, J=6.6 \mathrm{~Hz}, 1 \mathrm{H}), 4.70(\mathrm{~d}, J=6.6 \mathrm{~Hz}, 1 \mathrm{H}), 4.59$ (d, $J=7.2 \mathrm{~Hz}, 1 \mathrm{H}), 4.54(\mathrm{~d}$, $J=6.6 \mathrm{~Hz}, 1 \mathrm{H}), 4.12(\mathrm{dd}, J=7.2,5.4 \mathrm{~Hz}, 1 \mathrm{H}), 4.02(\mathrm{~s}, 2 \mathrm{H}), 4.00(\mathrm{q}, J=7.2 \mathrm{~Hz}, 1 \mathrm{H}), 3.40(\mathrm{~s}$, $3 \mathrm{H}), 3.38$ (s, $3 \mathrm{H}), 2.67$ (dqd, $J=9.6,6.6,5.4 \mathrm{~Hz}, 1 \mathrm{H}), 2.24$ (hex, $J=7.2 \mathrm{~Hz}, 4 \mathrm{H}$ ), 2.17 (pent, $J$ $=7.2 \mathrm{~Hz}, 2 \mathrm{H}), 1.78-1.51(\mathrm{~m}, 6 \mathrm{H}), 1.75(\mathrm{~s}, 3 \mathrm{H}), 1.66(\mathrm{~s}, 3 \mathrm{H}), 0.98 \mathrm{ppm}(\mathrm{d}, J=6.6 \mathrm{~Hz}, 3 \mathrm{H})$; ${ }^{13} \mathrm{C}$ NMR $\left(150 \mathrm{MHz}, \mathrm{CDCl}_{3}\right): \delta=166.3,156.5,148.8,138.1,136.1,134.0,132.3,131.6,127.6$, $127.1,126.5,121.6,119.4,117.4,94.0,93.7,77.8,75.3,75.2,68.7,55.7,55.5,42.4,35.6,34.8$, 32.0, 30.5, 28.7, 23.8, 17.1, 16.7, 14.0 ppm; HRMS (ESI): calcd for $\mathrm{C}_{33} \mathrm{H}_{53} \mathrm{NO}_{9} \mathrm{Na}^{+}\left[\mathrm{M}+\mathrm{Na}^{+}\right]$ 630.3612, found 630.3610 .

Aldehyde 62: To a solution of alcohol 61 (390 mg, $0.66 \mathrm{mmol})$ in $\mathrm{CH}_{2} \mathrm{Cl}_{2}(4.0 \mathrm{~mL})$ were added $\mathrm{NaHCO}_{3}(300 \mathrm{mg}, 3.57 \mathrm{mmol})$ and DMP $(416 \mathrm{mg}, 0.99 \mathrm{mmol})$ at room temperature. The reaction mixture was stirred for 30 min before it was quenched with $\mathrm{NaHCO}_{3}(10 \mathrm{~mL}$, sat. aq.) and $\mathrm{Na}_{2} \mathrm{~S}_{2} \mathrm{O}_{3}(10 \mathrm{~mL}$, sat. aq. $)$. The resulting mixture was extracted with EtOAc $(3 \times 30 \mathrm{~mL})$, and 


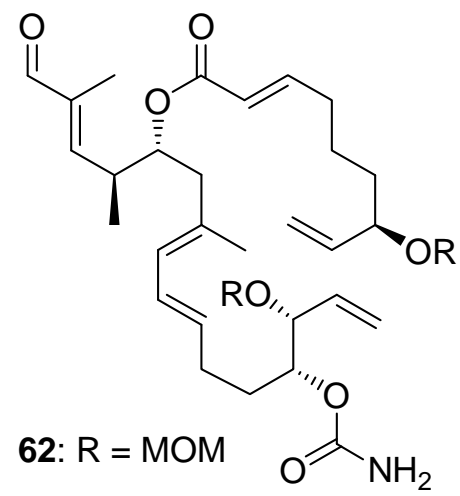

the combined organic layers were dried $\left(\mathrm{Na}_{2} \mathrm{SO}_{4}\right)$ and concentrated in vacuo. Flash column chromatography (silica gel, hexanes:EtOAc:Et ${ }_{3} \mathrm{~N}$ 4:3:0.01) afforded aldehyde 62 (316 mg, 81\%) as a colorless oil. 62: $R_{\mathrm{f}}=0.50$ (silica gel, hexanes:EtOAc 2:1); $[\alpha]_{\mathrm{D}}^{25}=+30.1\left(\mathrm{CH}_{2} \mathrm{Cl}_{2}, c=0.95\right)$; IR (film) $v_{\max } 3455,3363,2933$, $2857,1718,1688,1651,1448,1384,1318,1275,1150,1097,1031$ $920 \mathrm{~cm}^{-1} ;{ }^{1} \mathrm{H}$ NMR $\left(600 \mathrm{MHz}, \mathrm{CDCl}_{3}\right): \delta=9.43(\mathrm{~s}, 1 \mathrm{H}), 6.93(\mathrm{dt}, J=15.6,6.9 \mathrm{~Hz}, 1 \mathrm{H}), 6.46(\mathrm{~d}$, $J=9.6 \mathrm{~Hz}, 1 \mathrm{H}), 6.19(\mathrm{dd}, J=15.0,10.8 \mathrm{~Hz}, 1 \mathrm{H}), 5.81(\mathrm{~d}, J=15.6 \mathrm{~Hz}, 1 \mathrm{H}), 5.71$ (ddd, $J=17.4$, 10.8, 7.8 Hz, $1 \mathrm{H}), 5.70(\mathrm{~d}, J=15.6 \mathrm{~Hz}, 1 \mathrm{H}), 5.66(\mathrm{ddd}, J=18.0,10.8,7.8 \mathrm{~Hz}, 1 \mathrm{H}), 5.54(\mathrm{dt}, J$ $=15.0,7.2 \mathrm{~Hz}, 1 \mathrm{H}), 5.31(\mathrm{~d}, J=9.0 \mathrm{~Hz}, 1 \mathrm{H}), 5.32(\mathrm{~d}, J=18.6 \mathrm{~Hz}, 1 \mathrm{H}), 5.21(\mathrm{~d}, J=8.4 \mathrm{~Hz}, 1$ H), $5.20(\mathrm{~d}, J=18.0 \mathrm{~Hz}, 1 \mathrm{H}), 5.15(\mathrm{q}, J=6.0 \mathrm{~Hz}, 1 \mathrm{H}), 4.88(\mathrm{dt}, J=8.4,4.5 \mathrm{~Hz}, 1 \mathrm{H}), 4.84$ (brs, $2 \mathrm{H}), 4.71(\mathrm{~d}, J=6.6 \mathrm{~Hz}, 1 \mathrm{H}), 4.69(\mathrm{~d}, J=6.6 \mathrm{~Hz}, 1 \mathrm{H}), 4.57(\mathrm{~d}, J=6.6 \mathrm{~Hz}, 1 \mathrm{H}), 4.52(\mathrm{~d}, J=$ $6.6 \mathrm{~Hz}, 1 \mathrm{H}), 4.12$ (dd, $J=7.2,4.8 \mathrm{~Hz}, 1 \mathrm{H}), 3.99$ (q, $J=6.4 \mathrm{~Hz}, 1 \mathrm{H}), 3.38(\mathrm{~s}, 3 \mathrm{H}), 3.36(\mathrm{~s}, 3 \mathrm{H})$, $2.97(\mathrm{dqd}, J=10.2,6.6,6.0 \mathrm{~Hz}, 1 \mathrm{H}), 2.28(\mathrm{dd}, J=13.8,7.2 \mathrm{~Hz}, 1 \mathrm{H}), 2.23(\mathrm{q}, J=7.2 \mathrm{~Hz}, 4 \mathrm{H})$, 2.20-2.13 (m, $2 \mathrm{H}), 1.79-1.50(\mathrm{~m}, 5 \mathrm{H}), 1.70(\mathrm{~s}, 6 \mathrm{H}), 1.08 \mathrm{ppm}(\mathrm{d}, J=6.6 \mathrm{~Hz}, 3 \mathrm{H}) ;{ }^{13} \mathrm{C}$ NMR $\left(150 \mathrm{MHz}, \mathrm{CDCl}_{3}\right): \delta=195.3,166.0,156.6,154.2,149.5,140.0,138.1,134.0,132.2,131.3$ $128.2,126.8,121.1,119.4,117.4,94.0,93.7,77.8,76.9,75.2,74.2,55.7,55.4,43.1,36.8,34.8$ 32.0, 30.4, 28.7, 23.8, 16.6, 16.4, 9.4 ppm; HRMS (ESI): calcd for $\mathrm{C}_{33} \mathrm{H}_{51} \mathrm{NO}_{9} \mathrm{Na}^{+}\left[\mathrm{M}+\mathrm{Na}^{+}\right]$ 628.3456 , found 628.3450 .

Vinyl iodide 63: To a solution of $\mathrm{CrCl}_{2}(357 \mathrm{mg}, 2.90 \mathrm{mmol}$, flame-dried) in THF (3.5 mL) was added a solution of aldehyde $62(175 \mathrm{mg}, 0.29 \mathrm{mmol})$ and $\mathrm{CHI}_{3}(342 \mathrm{mg}, 0.87 \mathrm{mmol})$ in dioxane $(21 \mathrm{~mL})$ at room temperature. The reaction mixture was stirred for $2 \mathrm{~h}$ before it was quenched 


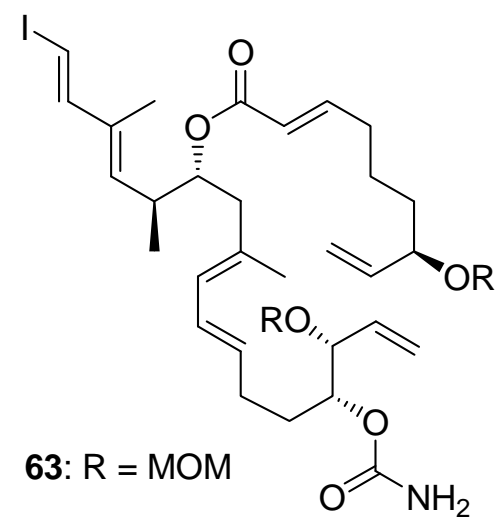

with $\mathrm{NaHCO}_{3}(25 \mathrm{~mL}$, sat. aq.). The resulting mixture was diluted with EtOAc $(100 \mathrm{~mL})$, filtered through a short pad of Celite ${ }^{\circledR}$ and washed with EtOAc $(100 \mathrm{~mL})$. The organic layer was separated, dried $\left(\mathrm{Na}_{2} \mathrm{SO}_{4}\right)$ and concentrated in vacuo. Flash column chromatography (silica gel, hexanes:EtOAc:Et ${ }_{3} \mathrm{~N} \quad 3: 2: 0.01$ ) afforded vinyl iodide $63(170 \mathrm{mg}, 81 \%)$ as a yellow oil. $63: R_{\mathrm{f}}=$ 0.30 (silica gel, hexanes:EtOAc 4:3); $[\alpha]_{\mathrm{D}}{ }^{25}=-6.2\left(\mathrm{CH}_{2} \mathrm{Cl}_{2}, c=0.70\right)$; IR (film) $v_{\max } 3360,2931$, 1716, 1652, 1603, 1441, 1385, 1317, 1149, 1096, 1030, $920 \mathrm{~cm}^{-1} ;{ }^{1} \mathrm{H}$ NMR (600 MHz, $\left.\mathrm{CDCl}_{3}\right)$ : $\delta=7.08(\mathrm{~d}, J=14.4 \mathrm{~Hz}, 1 \mathrm{H}), 6.92(\mathrm{dt}, J=15.6,6.9 \mathrm{~Hz}, 1 \mathrm{H}), 6.20(\mathrm{~d}, J=14.4 \mathrm{~Hz}, 1 \mathrm{H}), 6.19$ $(\mathrm{dd}, J=14.4,9.6 \mathrm{~Hz}, 1 \mathrm{H}), 5.80(\mathrm{~d}, J=15.6 \mathrm{~Hz}, 1 \mathrm{H}), 5.72(\mathrm{ddd}, J=16.8,10.2,7.2 \mathrm{~Hz}, 1 \mathrm{H})$, $5.72(\mathrm{~d}, J=10.2 \mathrm{~Hz}, 1 \mathrm{H}), 5.67(\mathrm{ddd}, J=18.0,10.8,7.8 \mathrm{~Hz}, 1 \mathrm{H}), 5.54(\mathrm{dt}, J=15.0,6.9 \mathrm{~Hz}, 1 \mathrm{H})$, $5.44(\mathrm{~d}, J=9.6 \mathrm{~Hz}, 1 \mathrm{H}), 5.33(\mathrm{~d}, J=9.0 \mathrm{~Hz}, 1 \mathrm{H}), 5.32(\mathrm{~d}, J=18.6 \mathrm{~Hz}, 1 \mathrm{H}), 5.23(\mathrm{~d}, J=8.4 \mathrm{~Hz}$, $1 \mathrm{H}), 5.22(\mathrm{~d}, J=18.6 \mathrm{~Hz}, 1 \mathrm{H}), 5.05(\mathrm{ddd}, J=7.8,6.0,4.2 \mathrm{~Hz}, 1 \mathrm{H}), 4.88(\mathrm{dt}, J=7.8,4.8 \mathrm{~Hz}, 1$ H), 4.75 (brs, $2 \mathrm{H}), 4.71(\mathrm{~d}, J=6.6 \mathrm{~Hz}, 1 \mathrm{H}), 4.70(\mathrm{~d}, J=6.0 \mathrm{~Hz}, 1 \mathrm{H}), 4.58(\mathrm{~d}, J=6.6 \mathrm{~Hz}, 1 \mathrm{H})$, $4.54(\mathrm{~d}, J=7.2 \mathrm{~Hz}, 1 \mathrm{H}), 4.12$ (dd, $J=6.6,5.4 \mathrm{~Hz}, 1 \mathrm{H}), 4.01$ (q, $J=6.4 \mathrm{~Hz}, 1 \mathrm{H}), 3.39$ (s, $3 \mathrm{H})$, $3.38(\mathrm{~s}, 3 \mathrm{H}), 2.73(\mathrm{dqd}, J=10.2,7.2,6.0 \mathrm{~Hz}, 1 \mathrm{H}), 2.27-2.20(\mathrm{~m}, 3 \mathrm{H}), 2.19-2.12(\mathrm{~m}, 3 \mathrm{H})$, 1.77-1.52 (m, $6 \mathrm{H}), 1.73(\mathrm{~s}, 3 \mathrm{H}), 1.72(\mathrm{~s}, 3 \mathrm{H}), 0.98 \mathrm{ppm}(\mathrm{d}, J=6.6 \mathrm{~Hz}, 3 \mathrm{H}) ;{ }^{13} \mathrm{C}$ NMR $(150$ $\left.\mathrm{MHz}, \mathrm{CDCl}_{3}\right): \delta=166.2,156.5,149.7,148.9,138.1,135.3,134.6,134.0,132.0,131.7,127.8$ 127.0, 121.5, 119.4, 117.4, 94.0, 93.7, 77.8, 77.0, 75.3, 74.8, 73.9, 55.7, 55.5, 42.6, 35.9, 34.8, 32.0, 30.4, 28.7, 23.8, 17.0, 16.7, 12.2 ppm; HRMS (ESI): calcd for $\mathrm{C}_{34} \mathrm{H}_{52} \mathrm{INO}_{8} \mathrm{Na}^{+}\left[\mathrm{M}+\mathrm{Na}^{+}\right]$ 752.2630 , found 752.2616 . 
Diol 64 and cyclic carbonate 65: To a solution of bis-MOM ether $63(113 \mathrm{mg}, 0.155 \mathrm{mmol})$ in EtOH $(5.0 \mathrm{~mL})$ was added $\mathrm{HCl}(3.0 \mathrm{~N}$ aq., $1.0 \mathrm{~mL}, 3.0 \mathrm{mmol})$ at room temperature. The reaction

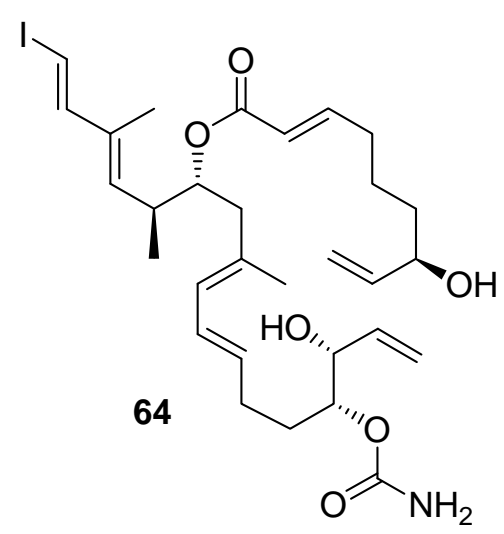
mixture was heated to $80^{\circ} \mathrm{C}$ and stirred for 50 min before it was cooled to room temperature and quenched with $\mathrm{NaHCO}_{3}(10 \mathrm{~mL}$, sat. aq.). The resulting mixture was extracted with EtOAc $(3 \times 30$ $\mathrm{mL})$, and the combined organic layers were dried $\left(\mathrm{Na}_{2} \mathrm{SO}_{4}\right)$ and concentrated in vacuo. Flash column chromatography (silica gel, hexanes:EtOAc: $\mathrm{Et}_{3} \mathrm{~N}$ 2:5:0.01) afforded diol $64(61 \mathrm{mg}, 62 \%)$ as a yellow oil and mono-deprotected product $(20 \mathrm{mg}, 18 \%)$ and cyclic carbonate 65 (12 mg, 11\%) as colorless oils. 64: $R_{\mathrm{f}}=0.22$ (silica gel, hexanes:EtOAc 1:1); $[\alpha]_{\mathrm{D}}{ }^{25}=-2.9\left(\mathrm{CH}_{2} \mathrm{Cl}_{2}, c=0.90\right.$ ); IR (film) $v_{\max } 3362,2930,2870,1710,1651,1390,1316,1248,1180,1062,991 \mathrm{~cm}^{-1} ;{ }^{1} \mathrm{H}$ NMR $\left(600 \mathrm{MHz}, \mathrm{CDCl}_{3}\right): \delta=7.09(\mathrm{~d}, J=15.0 \mathrm{~Hz}, 1 \mathrm{H}), 6.91(\mathrm{dt}, J=15.6,6.6 \mathrm{~Hz}, 1 \mathrm{H}), 6.21(\mathrm{~d}, J=$ $14.4 \mathrm{~Hz}, 1 \mathrm{H}), 6.19$ (dd, $J=14.4,10.8 \mathrm{~Hz}, 1 \mathrm{H}), 5.88$ (ddd, $J=17.4,10.8,6.6 \mathrm{~Hz}, 2 \mathrm{H}), 5.80$ (d, $J=15.6 \mathrm{~Hz}, 1 \mathrm{H}), 5.72(\mathrm{~d}, J=10.8 \mathrm{~Hz}, 1 \mathrm{H}), 5.52(\mathrm{dt}, J=15.0,6.9 \mathrm{~Hz}, 1 \mathrm{H}), 5.44(\mathrm{~d}, J=9.6 \mathrm{~Hz}$ $1 \mathrm{H}), 5.37$ (brd, $J=17.4 \mathrm{~Hz}, 1 \mathrm{H}), 5.25$ (brd, $J=15.6 \mathrm{~Hz}, 1 \mathrm{H}), 5.24(\mathrm{~d}, J=11.4 \mathrm{~Hz}, 1 \mathrm{H}), 5.13$ (brd, $J=10.2 \mathrm{~Hz}, 1 \mathrm{H}), 5.05(\mathrm{dt}, J=8.4,4.2 \mathrm{~Hz}, 1 \mathrm{H}), 4.81$ (brs, $2 \mathrm{H}), 4.74(\mathrm{q}, J=6.0 \mathrm{~Hz}, 1 \mathrm{H})$, 4.18-4.10 (m, $2 \mathrm{H}), 2.74$ (dqd, $J=9.6,6.6,5.4 \mathrm{~Hz}, 1 \mathrm{H}), 2.27-2.21$ (m, $3 \mathrm{H}), 2.20-2.13(\mathrm{~m}, 3 \mathrm{H})$, 1.79-1.51 (m, $6 \mathrm{H}), 1.73(\mathrm{~s}, 3 \mathrm{H}), 1.72(\mathrm{~s}, 3 \mathrm{H}), 0.99 \mathrm{ppm}(\mathrm{d}, J=6.6 \mathrm{~Hz}, 3 \mathrm{H}) ;{ }^{13} \mathrm{C}$ NMR $(150$ $\left.\mathrm{MHz}, \mathrm{CDCl}_{3}\right): \delta=166.2,157.0,149.6,148.9,141.0,136.9,135.2,134.7,132.1,131.5,127.8$ $127.2,121.5,117.0,114.8,76.9,74.7,74.2,74.0,72.8,42.7,36.4,36.1,32.0,30.4,28.7,23.8$, 17.0, 16.7, 12.2 ppm; HRMS (ESI): calcd for $\mathrm{C}_{30} \mathrm{H}_{44} \mathrm{NNO}_{6} \mathrm{Na}^{+}\left[\mathrm{M}+\mathrm{Na}^{+}\right]$664.2106, found 664.2088. 


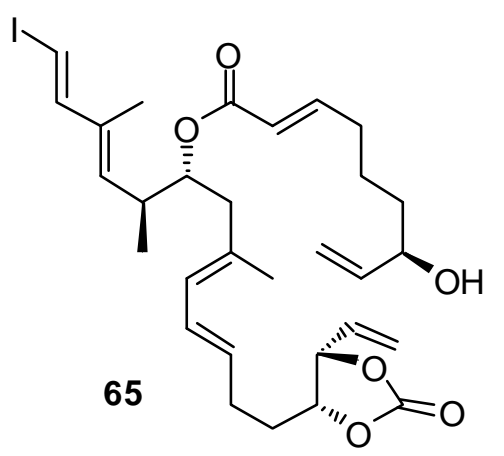

Cyclic carbonate 65: $R_{\mathrm{f}}=0.58$ (silica gel, hexanes:EtOAc 2:1); $[\alpha]_{\mathrm{D}}^{25}=-7.3\left(c=0.55, \mathrm{CH}_{2} \mathrm{Cl}_{2}\right) ; \mathrm{IR}($ film $) v_{\max } 3458,2931,1805$, $1712,1652,1432,1365,1275,1262,1187,1047,989,750 \mathrm{~cm}^{-1} ;{ }^{1} \mathrm{H}$ $\operatorname{NMR}\left(600 \mathrm{MHz}, \mathrm{CDCl}_{3}\right): \delta=7.09(\mathrm{~d}, J=15.0 \mathrm{~Hz}, 1 \mathrm{H}), 6.93(\mathrm{dt}, J$ $=15.6,6.6 \mathrm{~Hz}, 1 \mathrm{H}), 6.24(\mathrm{dd}, J=15.6,10.8 \mathrm{~Hz}, 1 \mathrm{H}), 6.21(\mathrm{~d}, J=$ $14.4 \mathrm{~Hz}, 1 \mathrm{H}), 5.88$ (ddd, $J=17.4,10.8,7.8 \mathrm{~Hz}, 2 \mathrm{H}), 5.80(\mathrm{~d}, J=15.6 \mathrm{~Hz}, 1 \mathrm{H}), 5.73$ (d, $J=$ $10.8 \mathrm{~Hz}, 1 \mathrm{H}), 5.50(\mathrm{~d}, J=17.4 \mathrm{~Hz}, 1 \mathrm{H}), 5.47$ (dt, $J=15.0,7.2 \mathrm{~Hz}, 1 \mathrm{H}), 5.44(\mathrm{~d}, J=10.8 \mathrm{~Hz}, 2$ H), $5.25(\mathrm{brd}, J=17.4 \mathrm{~Hz}, 1 \mathrm{H}), 5.14(\mathrm{~d}, J=10.2 \mathrm{~Hz}, 1 \mathrm{H}), 5.06(\mathrm{dt}, J=6.6,5.4 \mathrm{~Hz}, 1 \mathrm{H}), 4.66(\mathrm{t}$, $J=7.2 \mathrm{~Hz}, 1 \mathrm{H}), 4.33(\mathrm{td}, J=7.8,4.2 \mathrm{~Hz}, 1 \mathrm{H}), 4.16-4.10(\mathrm{~m}, 1 \mathrm{H}), 2.73(\mathrm{ddq}, J=10.8,7.2,6.6$ Hz, 1 H), 2.32-2.16 (m, 6 H), 1.91-1.85 (m, 1 H), 1.81-1.68 (m, 2 H), 1.75 (s, 3 H), 1.72 (s, 3 $\mathrm{H}), 1.65-1.51(\mathrm{~m}, 3 \mathrm{H}), 1.00 \mathrm{ppm}(\mathrm{d}, J=6.6 \mathrm{~Hz}, 3 \mathrm{H}) ;{ }^{13} \mathrm{C} \mathrm{NMR}\left(150 \mathrm{MHz}, \mathrm{CDCl}_{3}\right): \delta=166.2$, 154.2, 149.6, 149.0, 141.0, 135.3, 134.6, 133.2, 132.0, 129.6, 128.3, 127.2, 121.5, 121.4, 114.9, 82.6, 81.1, 74.7, 74.0, 72.9, 42.6, 36.3, 36.0, 32.8, 32.0, 28.0, 23.8, 17.0, 16.7, 12.2 ppm; HRMS (ESI): calcd for $\mathrm{C}_{30} \mathrm{H}_{41} \mathrm{IO}_{6} \mathrm{Na}^{+}\left[\mathrm{M}+\mathrm{Na}^{+}\right]$647.1840, found 647.1848.

Macrocycle 66: To a solution of diol $64(61 \mathrm{mg}, 0.095 \mathrm{mmol})$ in $\mathrm{CH}_{2} \mathrm{Cl}_{2}(50 \mathrm{~mL})$ was added Grubbs II catalyst (16 mg, $0.019 \mathrm{mmol})$ at room temperature. The mixture was stirred for $2 \mathrm{~h}$ before it was concentrated in vacuo. Flash column chromatography (silica gel, MeOH:EtOAc:Et ${ }_{3} \mathrm{~N}$ 2:100:1) afforded macrocycle 66 (38 mg, 66\%) as a colorless oil. 66: $R_{\mathrm{f}}=$ 0.34 (silica gel, hexanes:EtOAc 0:1); $[\alpha]_{\mathrm{D}}{ }^{25}=-6.7\left(\mathrm{CH}_{2} \mathrm{Cl}_{2}, c=0.60\right)$; IR (film) $v_{\max } 3358,2926$, 2856, 1714, 1655, 1447, 1392, 1315, 1261, 1186, 1077, $975 \mathrm{~cm}^{-1} ;{ }^{1} \mathrm{H}$ NMR (600 MHz, $\mathrm{CDCl}_{3}$ ): $\delta=7.07(\mathrm{~d}, J=14.4 \mathrm{~Hz}, 1 \mathrm{H}), 6.82(\mathrm{ddd}, J=15.3,10.5,5.4 \mathrm{~Hz}, 1 \mathrm{H}), 6.20(\mathrm{~d}, J=14.4 \mathrm{~Hz}, 1 \mathrm{H})$, $6.13(\mathrm{dd}, J=15.0,10.2 \mathrm{~Hz}, 1 \mathrm{H}), 5.74(\mathrm{dd}, J=15.6,3.0 \mathrm{~Hz}, 1 \mathrm{H}), 5.73(\mathrm{~d}, J=15.0 \mathrm{~Hz}, 1 \mathrm{H})$, 


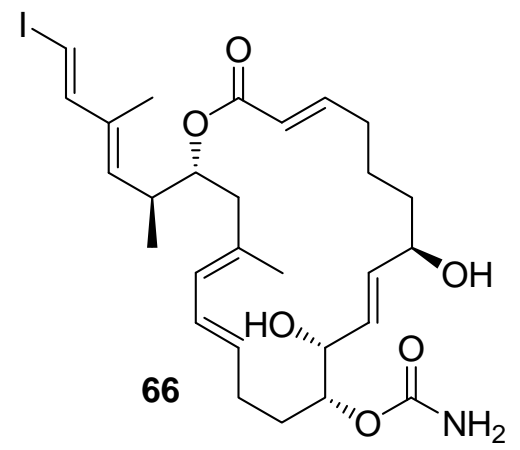

$5.67(\mathrm{dd}, J=15.6,7.8 \mathrm{~Hz}, 1 \mathrm{H}), 5.65(\mathrm{~d}, J=10.8 \mathrm{~Hz}, 1 \mathrm{H}), 5.40$ (ddd, $J=14.4,9.6,4.8 \mathrm{~Hz}, 1 \mathrm{H}), 5.39(\mathrm{~d}, J=9.6 \mathrm{~Hz}, 1 \mathrm{H})$, $5.18-5.05(\mathrm{~m}, 3 \mathrm{H}), 4.68$ (td, $J=8.4,4.0 \mathrm{~Hz}, 1 \mathrm{H}), 4.32$ (brs, 1 H), 4.03 (t, $J=7.8 \mathrm{~Hz}, 1 \mathrm{H}), 3.54$ (brs, $1 \mathrm{H}), 2.80$ (brs, $1 \mathrm{H}), 2.72$ (ddq, $J=11.4,6.6,6.6 \mathrm{~Hz}, 1 \mathrm{H}), 2.25-2.08$ (m, $4 \mathrm{H}$ ), 2.06-1.82 (m, $3 \mathrm{H}), 1.75(\mathrm{~s}, 3 \mathrm{H}), 1.71(\mathrm{~s}, 3 \mathrm{H}), 1.66-1.57(\mathrm{~m}, 1 \mathrm{H}), 1.52-1.43(\mathrm{~m}, 3 \mathrm{H}), 1.34-1.25(\mathrm{~m}, 1$ H), $1.02 \mathrm{ppm}(\mathrm{d}, J=6.6 \mathrm{~Hz}, 3 \mathrm{H}) ;{ }^{13} \mathrm{C} \mathrm{NMR}\left(150 \mathrm{MHz}, \mathrm{CDCl}_{3}\right): \delta=166.1,157.6,149.6,148.9$, 137.3, 135.1, 135.1, 131.8, 131.4, 128.0, 127.2, 127.2, 121.3, 77.9, 76.1, 74.0, 73.4, 70.3, 43.4, 37.0, 36.1, 32.9, 31.9, 28.8, 23.3, 17.0, 16.4, 12.2 ppm; HRMS (ESI): calcd for $\mathrm{C}_{28} \mathrm{H}_{40} \mathrm{INO}_{6} \mathrm{Na}^{+}$ $\left[\mathrm{M}+\mathrm{Na}^{+}\right]$636.1792, found 636.1774.

Palmerolide A isomer 20-epi-1 and decarbamated palmerolide A isomer 67: To a mixture of vinyl iodide 66 ( $9.0 \mathrm{mg}, 0.015 \mathrm{mmol})$, amide 42 (3.0 mg, $0.03 \mathrm{mmol})$, CuI (2.9 mg, $0.015 \mathrm{mmol})$

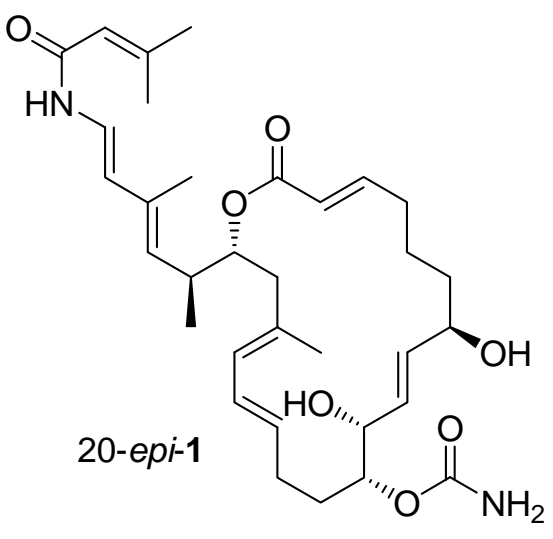
and $\mathrm{K}_{2} \mathrm{CO}_{3}$ (10.0 mg, $0.075 \mathrm{mmol}$, flame dried) in DMF (1.3 $\mathrm{mL})$ was added $N, N^{\prime}$-dimethylethylenediamine $(5.0 \mu \mathrm{L}, 0.045$ $\mathrm{mmol}$ ) at room temperature. The resulting mixture was stirred for $1 \mathrm{~h}$ before it was diluted with EtOAc $(20 \mathrm{~mL})$, filtered through a short pad of Celite ${ }^{\circledR}$ and concentrated in vacuo. Preparative TLC purification (silica gel, hexanes:EtOAc 0:1) afforded 20-epi-1 (3.4 mg, 40\%) as a colorless oil and decarbamated product 67 (0.9 mg, 11\%) as a colorless oil. Palmerolide A isomer 20-epi-1: $R_{\mathrm{f}}=0.25$ (silica gel, hexanes:EtOAc 0:1); $[\alpha]_{\mathrm{D}}{ }^{25}=+4.7(\mathrm{MeOH}, c=0.17)$; IR (film) $v_{\max } 3348,2925,2855,1714,1639,1521,1392,1315$, 1191, 1154, $1077 \mathrm{~cm}^{-1} ;{ }^{1} \mathrm{H}$ NMR $\left(600 \mathrm{MHz}, \mathrm{DMSO}-d^{6}\right): \delta=9.84(\mathrm{~d}, J=10.2 \mathrm{~Hz}, 1 \mathrm{H}), 6.83(\mathrm{dd}$, 
$J=14.4,10.2 \mathrm{~Hz}, 1 \mathrm{H}), 6.67$ (ddd, $J=15.0,9.0,6.0 \mathrm{~Hz}, 1 \mathrm{H}), 6.38$ (brs, $2 \mathrm{H}$ ), 6.09 (dd, $J=14.7$, $10.8 \mathrm{~Hz}, 1 \mathrm{H}), 5.82(\mathrm{~d}, J=15.0 \mathrm{~Hz}, 1 \mathrm{H}), 5.74(\mathrm{~d}, J=15.6 \mathrm{~Hz}, 1 \mathrm{H}), 5.68(\mathrm{~s}, 1 \mathrm{H}), 5.59(\mathrm{~d}, J=$ $10.2 \mathrm{~Hz}, 1 \mathrm{H}), 5.58$ (dd, $J=15.6,4.8 \mathrm{~Hz}, 1 \mathrm{H}), 5.48$ (dd, $J=15.6,7.2 \mathrm{~Hz}, 1 \mathrm{H}), 5.39$ (ddd, $J=$ 15.0, 9.6, $5.4 \mathrm{~Hz}, 1 \mathrm{H}), 5.13(\mathrm{~d}, J=9.6 \mathrm{~Hz}, 1 \mathrm{H}), 4.95(\mathrm{ddd}, J=11.4,4.8,1.8 \mathrm{~Hz}, 1 \mathrm{H}), 4.92(\mathrm{~d}, J$ $=5.4 \mathrm{~Hz}, 1 \mathrm{H}), 4.72(\mathrm{~d}, J=4.8 \mathrm{~Hz}, 1 \mathrm{H}), 4.48(\mathrm{ddd}, J=10.2,7.2,3.0 \mathrm{~Hz}, 1 \mathrm{H}), 4.02(\mathrm{~m}, 1 \mathrm{H})$, $3.89(\mathrm{q}, J=6.4 \mathrm{~Hz}, 1 \mathrm{H}), 2.74(\mathrm{dqd}, J=9.6,7.2,4.8 \mathrm{~Hz}, 1 \mathrm{H}), 2.19-1.96(\mathrm{~m}, 3 \mathrm{H}), 2.11(\mathrm{~s}, 3 \mathrm{H})$, 1.92-1.76 (m, 2 H), 1.83 (s, 3 H), 1.73-1.60 (m, 1 H), 1.69 (s, 3 H), 1.63 (s, 3 H), 1.41-1.31 (m, $3 \mathrm{H}), 1.28-1.16(\mathrm{~m}, 3 \mathrm{H}), 0.93 \mathrm{ppm}(\mathrm{d}, J=6.6 \mathrm{~Hz}, 3 \mathrm{H}) ;{ }^{13} \mathrm{C}$ NMR $\left(150 \mathrm{MHz}\right.$, DMSO- $\left.d^{6}\right): \delta=$ $165.7,163.7,157.4,151.9,149.2,135.9,133.1,132.8,131.6,130.0,128.7,128.3,126.8,122.5$, 121.6, 118.7, 117.0, 76.1, 74.0, 73.2, 70.2, 42.9, 37.4, 36.9, 32.6, 31.7, 28.9, 27.4, 23.9, 20.0, 17.6, 16.7, 13.0 ppm; HRMS (ESI): calcd for $\mathrm{C}_{33} \mathrm{H}_{48} \mathrm{~N}_{2} \mathrm{O}_{7} \mathrm{Na}^{+}\left[\mathrm{M}+\mathrm{Na}^{+}\right] 607.3354$, found 607.3343 .

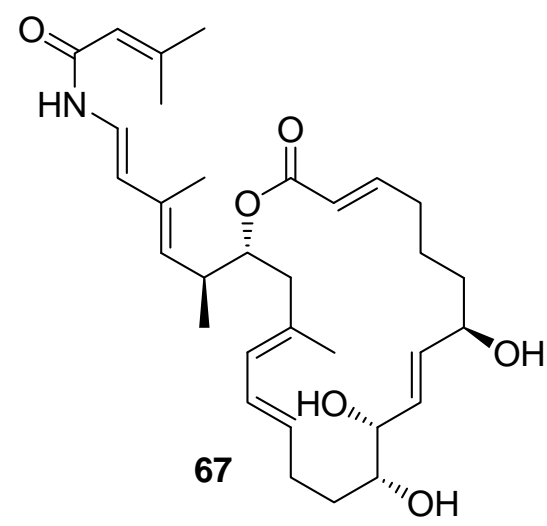

Decarbamated palmerolide A isomer 67: $R_{\mathrm{f}}=0.28$ (silica gel, EtOAc); $[\alpha]_{\mathrm{D}}^{25}=+13.5(\mathrm{MeOH}, c=0.20) ; \mathrm{IR}($ film $) v_{\max } 3361$, 2926, 1714, 1640, 1522, 1392, 1315, 1194, 1154, 1077, 976 $\mathrm{cm}^{-1} ;{ }^{1} \mathrm{H}$ NMR $\left(600 \mathrm{MHz}\right.$, DMSO- $\left.d^{6}\right): \delta=9.82(\mathrm{~d}, J=10.8$ $\mathrm{Hz}, 1 \mathrm{H}), 6.83(\mathrm{dd}, J=14.4,10.2 \mathrm{~Hz}, 1 \mathrm{H}), 6.68(\mathrm{dt}, J=15.6$, $7.8 \mathrm{~Hz}, 1 \mathrm{H}), 6.08(\mathrm{dd}, J=15.0,11.4 \mathrm{~Hz}, 1 \mathrm{H}), 5.83(\mathrm{~d}, J=$ $14.4 \mathrm{~Hz}, 1 \mathrm{H}), 5.74(\mathrm{~d}, J=16.2 \mathrm{~Hz}, 1 \mathrm{H}), 5.69(\mathrm{~s}, 1 \mathrm{H}), 5.60(\mathrm{~d}, J=10.8 \mathrm{~Hz}, 1 \mathrm{H}), 5.53(\mathrm{dd}, J=$ 15.6, 3.6 Hz, $1 \mathrm{H}), 5.49$ (dd, $J=15.6,3.6 \mathrm{~Hz}, 1 \mathrm{H}), 5.40$ (ddd, $J=15.0,10.2,4.8 \mathrm{~Hz}, 1 \mathrm{H}), 5.13$ (d, $J=9.6 \mathrm{~Hz}, 1 \mathrm{H}), 4.97$ (ddd, $J=11.4,4.8,1.8 \mathrm{~Hz}, 1 \mathrm{H}), 4.67$ (brs, $2 \mathrm{H}$ ), 4.46 (brs, $1 \mathrm{H}$ ), 4.04 (m, $1 \mathrm{H}), 3.68(\mathrm{~m}, 1 \mathrm{H}), 3.18(\mathrm{~m}, 1 \mathrm{H}), 2.74(\mathrm{dqd}, J=9.6,7.2,5.4 \mathrm{~Hz}, 1 \mathrm{H}), 2.22-1.99(\mathrm{~m}, 6 \mathrm{H})$, $2.10(\mathrm{~s}, 3 \mathrm{H}), 1.91-1.79(\mathrm{~m}, 1 \mathrm{H}), 1.83(\mathrm{~s}, 3 \mathrm{H}), 1.70(\mathrm{~s}, 3 \mathrm{H}), 1.63(\mathrm{~s}, 3 \mathrm{H}), 1.41-1.30(\mathrm{~m}, 2 \mathrm{H})$, 
1.27-1.18 (m, $2 \mathrm{H}), 1.15-1.09$ (m, $1 \mathrm{H}), 0.93 \mathrm{ppm}(\mathrm{d}, J=7.2 \mathrm{~Hz}, 3 \mathrm{H}) ;{ }^{13} \mathrm{C}$ NMR $(150 \mathrm{MHz}$ DMSO- $\left.d^{6}\right): \delta=165.7,163.6,152.1,149.5,135.4,133.7,133.1,131.3,130.1,129.3,128.3,126.6$, 122.4, 121.4, 118.6, 117.0, 76.6, 73.9, 69.8, 43.1, 37.2, 36.9, 33.9, 32.7, 29.7, 27.4, 23.8, 20.0, 17.7, 16.6, 13.0 ppm; HRMS (ESI): calcd for $\mathrm{C}_{32} \mathrm{H}_{47} \mathrm{NO}_{6} \mathrm{Na}^{+}\left[\mathrm{M}+\mathrm{Na}^{+}\right] 564.3296$, found 564.3298.

Triol 68: To a solution of cyclic carbonate $65(19 \mathrm{mg}, 0.03 \mathrm{mmol})$ in $\mathrm{MeOH}(0.75 \mathrm{~mL})$ was added $\mathrm{NaOMe}(24 \mathrm{mg}, 0.45 \mathrm{mmol})$ at room temperature. The reaction mixture was stirred for $2 \mathrm{~h}$

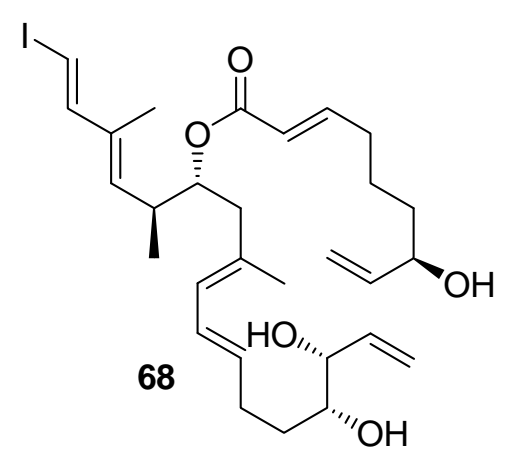
before it was quenched with $\mathrm{NH}_{4} \mathrm{Cl}(20 \mathrm{~mL}$, sat. aq.) The resulting mixture was extracted with EtOAc $(3 \times 30 \mathrm{~mL})$, and the combined organic layers were dried $\left(\mathrm{Na}_{2} \mathrm{SO}_{4}\right)$ and concentrated in vacuo. Flash column chromatography (silica gel, hexanes:EtOAc: $\mathrm{Et}_{3} \mathrm{~N}$ 2:5:0.01) afforded triol $68(10 \mathrm{mg}, 55 \%)$ as a colorless oil. 68: $R_{\mathrm{f}}=0.30$ (silica gel, hexanes:EtOAc 1:1); $[\alpha]_{\mathrm{D}}{ }^{25}=-13.8\left(\mathrm{CH}_{2} \mathrm{Cl}_{2}, c=0.50\right.$ ); IR (film) $v_{\max } 3406,2928,1714,1649,1438,1389,1275,1262,1179,991,750 \mathrm{~cm}^{-1} ;{ }^{1} \mathrm{H}$ NMR $\left(600 \mathrm{MHz}, \mathrm{CDCl}_{3}\right): \delta=7.09(\mathrm{~d}, J=14.4 \mathrm{~Hz}, 1 \mathrm{H}), 6.92(\mathrm{dt}, J=15.6,6.9 \mathrm{~Hz}, 1 \mathrm{H}), 6.23(\mathrm{dd}, J=$ 14.4, $10.8 \mathrm{~Hz}, 1 \mathrm{H}), 6.22$ (d, $J=14.4 \mathrm{~Hz}, 1 \mathrm{H}), 5.87$ (ddd, $J=17.4,11.4,6.6 \mathrm{~Hz}, 2 \mathrm{H}), 5.80(\mathrm{~d}, J$ $=15.6 \mathrm{~Hz}, 1 \mathrm{H}), 5.73(\mathrm{~d}, J=10.8 \mathrm{~Hz}, 1 \mathrm{H}), 5.55(\mathrm{dt}, J=15.0,7.5 \mathrm{~Hz}, 1 \mathrm{H}), 5.44(\mathrm{~d}, J=9.6 \mathrm{~Hz}, 1$ H), $5.37(\mathrm{~d}, J=16.8 \mathrm{~Hz}, 1 \mathrm{H}), 5.26(\mathrm{~d}, J=10.2 \mathrm{~Hz}, 1 \mathrm{H}), 5.25(\mathrm{brd}, J=16.8 \mathrm{~Hz}, 1 \mathrm{H}), 5.14(\mathrm{~d}, J$ $=10.2 \mathrm{~Hz}, 1 \mathrm{H}), 5.06(\mathrm{dt}, J=7.2,5.4 \mathrm{~Hz}, 1 \mathrm{H}), 4.16-4.10(\mathrm{~m}, 1 \mathrm{H}), 3.95(\mathrm{t}, J=5.7 \mathrm{~Hz}, 1 \mathrm{H})$, $3.50(\mathrm{td}, J=6.0,3.6 \mathrm{~Hz}, 1 \mathrm{H}), 3.33(\mathrm{~d}, J=2.4 \mathrm{~Hz}, 1 \mathrm{H}), 2.74(\mathrm{ddq}, J=10.8,6.6,6.6 \mathrm{~Hz}, 1 \mathrm{H})$, 2.35-2.15 (m, $6 \mathrm{H}), 1.65-1.52(\mathrm{~m}, 6 \mathrm{H}), 1.75$ (s, $3 \mathrm{H}), 1.74$ (s, $3 \mathrm{H}), 0.96 \mathrm{ppm}(\mathrm{d}, J=7.2 \mathrm{~Hz}, 3$ $\mathrm{H}) ;{ }^{13} \mathrm{C} \mathrm{NMR}\left(150 \mathrm{MHz}, \mathrm{CDCl}_{3}\right): \delta=166.2,149.7,148.9,141.0,137.6,135.2,134.7,132.2$, 
132.0, 127.8, 127.1, 121.5, 117.5, 114.9, 76.1, 74.6, 73.9, 73.7, 72.9, 42.7, 36.3, 36.0, 32.6, 32.0, 28.9, 23.9, 17.0, 16.6, 12.2 ppm; HRMS (ESI): calcd for $\mathrm{C}_{29} \mathrm{H}_{43} \mathrm{IO}_{5} \mathrm{Na}^{+}\left[\mathrm{M}+\mathrm{Na}^{+}\right]$621.2047, found 621.2042 .

Macrocycle 69: To a solution of triol $68(10 \mathrm{mg}, 0.0168 \mathrm{mmol})$ in $\mathrm{CH}_{2} \mathrm{Cl}_{2}(8.4 \mathrm{~mL})$ was added Grubbs II catalyst $(2.8 \mathrm{mg}, 0.0034 \mathrm{mmol})$ at room temperature. The reaction mixture was stirred

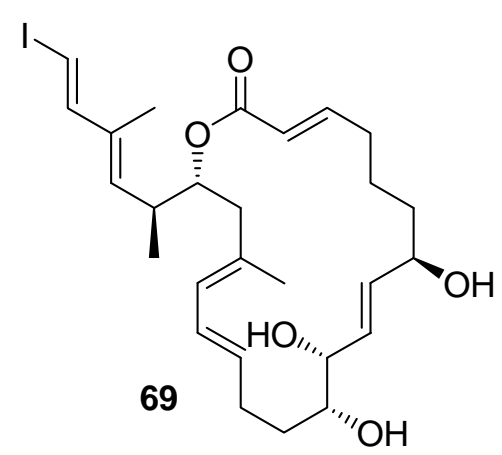
for $2 \mathrm{~h}$ before it was concentrated in vacuo. Preparative TLC purification (silica gel hexanes:EtOAc 0:1) afforded macrocycle $69(6.0 \mathrm{mg}, 63 \%)$ as a colorless oil. $69: R_{\mathrm{f}}=0.46$ (silica gel, hexanes:EtOAc 0:1); $[\alpha]_{\mathrm{D}}{ }^{25}=-38.1\left(\mathrm{CH}_{2} \mathrm{Cl}_{2, c}=0.26\right)$; IR (film) $v_{\max } 3385,2929,2858,1716,1654,1446,1352,1275,1261,1186$, 1078, 975, $751 \mathrm{~cm}^{-1} ;{ }^{1} \mathrm{H}$ NMR $\left(600 \mathrm{MHz}, \mathrm{CDCl}_{3}\right): \delta=7.07(\mathrm{~d}, J=15.0 \mathrm{~Hz}, 1 \mathrm{H}), 6.83(\mathrm{ddd}, J=$ 15.0, 8.4, $4.8 \mathrm{~Hz}, 1 \mathrm{H}), 6.21(\mathrm{~d}, J=14.4 \mathrm{~Hz}, 1 \mathrm{H}), 6.17$ (dd, $J=15.6,10.8 \mathrm{~Hz}, 1 \mathrm{H}), 5.76(\mathrm{dd}, J=$ 15.6, 3.6 Hz, $1 \mathrm{H}), 5.70$ (d, $J=15.6 \mathrm{~Hz}, 1 \mathrm{H}), 5.67$ (dd, $J=15.6,7.8 \mathrm{~Hz}, 1 \mathrm{H}), 5.66$ (d, $J=9.6$ $\mathrm{Hz}, 1 \mathrm{H}), 5.43(\mathrm{ddd}, J=15.0,10.8,4.8 \mathrm{~Hz}, 1 \mathrm{H}), 5.39$ (d, $J=10.2 \mathrm{~Hz}, 1 \mathrm{H}), 5.11$ (dt, $J=7.8,5.4$ Hz, $1 \mathrm{H}), 4.37$ (m, $1 \mathrm{H}), 3.87$ (t, $J=7.8 \mathrm{~Hz}, 1 \mathrm{H}), 3.44$ (t, $J=8.4 \mathrm{~Hz}, 1 \mathrm{H}), 2.72$ (dqd, $J=12.0$, 7.2, $6.6 \mathrm{~Hz}, 1 \mathrm{H}), 2.38-2.22(\mathrm{~m}, 2 \mathrm{H}), 2.21-2.12(\mathrm{~m}, 3 \mathrm{H}), 2.07-1.99(\mathrm{~m}, 1 \mathrm{H}), 1.75(\mathrm{~s}, 3 \mathrm{H})$, $1.73(\mathrm{~s}, 3 \mathrm{H}), 1.54-1.49$ (m, $2 \mathrm{H}), 1.48-1.40(\mathrm{~m}, 1 \mathrm{H}), 1.36-1.27$ (m, $3 \mathrm{H}), 1.02$ ppm (d, $J=6.6$ $\mathrm{Hz}, 3 \mathrm{H}) ;{ }^{13} \mathrm{C}$ NMR $\left(150 \mathrm{MHz}, \mathrm{CDCl}_{3}\right): \delta=166.0,149.6,148.7,137.0,135.1,132.1,131.6$, $128.1,127.8,127.1,121.3,77.5,74.2,73.9,73.4,70.4,43.4,37.0,36.1,33.5,32.8,29.3,23.2$ 16.9, 16.4, 12.2 ppm; HRMS (ESI): calcd for $\mathrm{C}_{27} \mathrm{H}_{39} \mathrm{IO}_{5} \mathrm{Na}^{+}\left[\mathrm{M}+\mathrm{Na}^{+}\right]$593.1734, found 593.1734. 
Enamide 67: To a mixture of vinyl iodide 69 (6.0 mg, $0.0106 \mathrm{mmol})$, amide 42 (2.1 mg, 0.021 mmol), $\mathrm{CuI}(2.0 \mathrm{mg}, 0.0106 \mathrm{mmol})$ and $\mathrm{K}_{2} \mathrm{CO}_{3}(7.2 \mathrm{mg}, 0.053 \mathrm{mmol}$, flame dried) in DMF (1.0

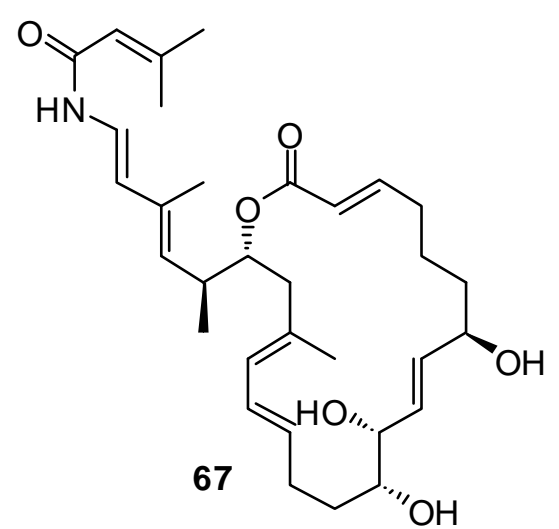
$\mathrm{mL})$ was added $N, N^{\prime}$-dimethylethylenediamine $(3.5 \mu \mathrm{L}, 0.032$ mmol) at room temperature. The reaction mixture was stirred for $1 \mathrm{~h}$ before it was diluted with EtOAc $(20 \mathrm{~mL})$, filtered through a short pad of Celite ${ }^{\circledR}$ and concentrated in vacuo. Preparative TLC purification (silica gel, hexanes:EtOAc 1:8) afforded enamide $67(2.4 \mathrm{mg}, 42 \%)$ as a colorless oil. All physical properties of this compound were identical to those reported for decarbamated product 67 obtained from vinyl iodide 66.

Alcohol 70: To a solution of TBS ether $51(450 \mathrm{mg}, 0.62 \mathrm{mmol})$ in THF $(10 \mathrm{~mL})$ was added TBAF (1.0 M in THF, $0.93 \mathrm{~mL}, 0.93 \mathrm{mmol})$ at room temperature. The reaction mixture was

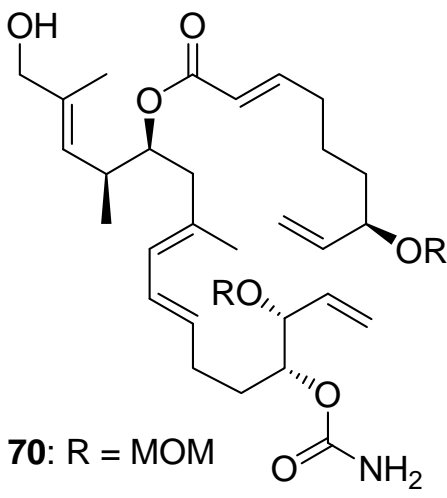
stirred for $2 \mathrm{~h}$ before it was concentrated in vacuo. The resulting residue was diluted with water $(5 \mathrm{~mL})$ and extracted with EtOAc $(3$ $\times 20 \mathrm{~mL})$. The combined organic layers were washed with brine $(5$ $\mathrm{mL})$, dried $\left(\mathrm{Na}_{2} \mathrm{SO}_{4}\right)$ and concentrated in vacuo. Flash column chromatorgraphy (silica gel, hexanes:EtOAc:Et ${ }_{3} \mathrm{~N}$ 7:3:1) afforded alcohol $70(323 \mathrm{mg}, 77 \%)$ as a viscous oil. 70: $R_{\mathrm{f}}=0.35$ (silica gel, hexanes:EtOAc 3:2); $[\alpha]_{\mathrm{D}}^{25}=-19.6\left(\mathrm{CHCl}_{3}, c=1.9\right)$; IR (film) $v_{\max } 3455,2932,1714,1386$, 1319, 1268, $1029 \mathrm{~cm}^{-1} ;{ }^{1} \mathrm{H}$ NMR $\left(600 \mathrm{MHz}, \mathrm{CDCl}_{3}\right): \delta=6.92(\mathrm{dt}, J=13.8,6.9 \mathrm{~Hz}, 1 \mathrm{H}), 6.20$ $(\mathrm{dd}, J=15.0,10.8 \mathrm{~Hz}, 1 \mathrm{H}), 5.80(\mathrm{dt}, 15.7,1.4 \mathrm{~Hz}, 1 \mathrm{H}), 5.75(\mathrm{~d}, J=12.0 \mathrm{~Hz}, 1 \mathrm{H}), 5.72(\mathrm{ddd}, J$ $=17.7,10.3,7.3 \mathrm{~Hz}, 1 \mathrm{H}), 5.67(\mathrm{ddd}, J=18.0,10.5,7.7 \mathrm{~Hz}, 1 \mathrm{H}), 5.53(\mathrm{dt} J=14.3,6.9 \mathrm{~Hz}, 1 \mathrm{H})$, 
$5.33(\mathrm{dd}, J=8.6,1.0 \mathrm{~Hz}, 1 \mathrm{H}), 5.32(\mathrm{~d}, J=1.0 \mathrm{~Hz}, 1 \mathrm{H}), 5.26(\mathrm{dd}, J=9.9,1.2 \mathrm{~Hz}, 1 \mathrm{H}), 5.23$ (bm, $1 \mathrm{H}), 5.20$ (brs, $1 \mathrm{H}), 4.98$ (ddd, $J=11.1,6.9,4.4 \mathrm{~Hz}, 1 \mathrm{H}), 4.88(\mathrm{dt}, J=9.0,4.8 \mathrm{~Hz}, 1 \mathrm{H})$, $4.71(\mathrm{~d}, J=4.0 \mathrm{~Hz}, 1 \mathrm{H}), 4.70(\mathrm{~d}, J=4.0 \mathrm{~Hz}, 1 \mathrm{H}), 4.73-4.65(\mathrm{brs}, 2 \mathrm{H}), 4.59(\mathrm{~d}, J=6.8 \mathrm{~Hz}, 1$ H), $4.54(\mathrm{~d}, J=6.8 \mathrm{~Hz}, 1 \mathrm{H}), 4.12(\mathrm{bm}, 1 \mathrm{H}), 4.00(\mathrm{brs}, 3 \mathrm{H}), 3.40(\mathrm{~s}, 3 \mathrm{H}), 3.38(\mathrm{~s}, 3 \mathrm{H}), 2.67$ (ddq, $J=12.0,11.1,6.8 \mathrm{~Hz}, 1 \mathrm{H}), 2.32-2.13(\mathrm{~m}, 6 \mathrm{H}), 1.74(\mathrm{~s}, 3 \mathrm{H}), 1.66(\mathrm{~d}, J=1.1 \mathrm{~Hz}, 3 \mathrm{H})$, $1.75-1.50(\mathrm{~m}, 7 \mathrm{H}), 0.98 \mathrm{ppm}(\mathrm{d}, J=6.8 \mathrm{~Hz}, 3 \mathrm{H}) ;{ }^{13} \mathrm{C} \mathrm{NMR}\left(150 \mathrm{MHz}, \mathrm{CDCl}_{3}\right): \delta=166.3$, 156.6, 148.8, 138.1, 135.6, 134.0, 132.5, 131.5, 127.6, 127.3, 127.2, 121.6, 119.4, 117.4, 94.0, $93.7,77.8,77.0,75.5,75.3,68.6,55.7,55.4,42.6,36.0,34.8,32.0,30.4,28.7,23.8,16.7,16.4$, 14.0 ppm; HRMS (ESI): calcd for $\mathrm{C}_{33} \mathrm{H}_{53} \mathrm{NO}_{9} \mathrm{Na}^{+}\left[\mathrm{M}+\mathrm{Na}^{+}\right]$630.3613, found 630.3630 .

Aldehyde 71: To a solution of alcohol 70 (270 mg, $0.464 \mathrm{mmol})$ in $\mathrm{CH}_{2} \mathrm{Cl}_{2}(3.0 \mathrm{~mL})$ was added $\mathrm{NaHCO}_{3}(242 \mathrm{mg}, 2.88 \mathrm{mmol})$ followed by DMP $(295 \mathrm{mg}, 0.696 \mathrm{mmol})$ at room temperature.

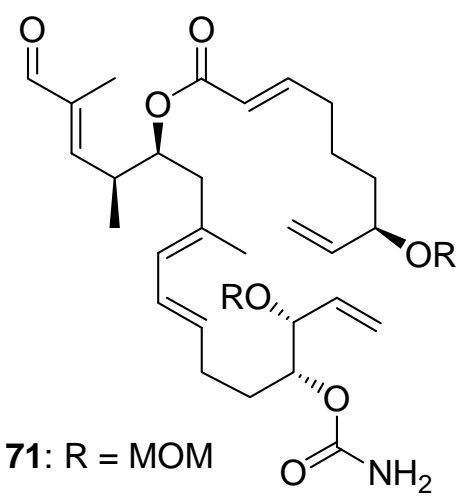
The reaction mixture was stirred for $30 \mathrm{~min}$ before it was quenched with $\mathrm{NaHCO}_{3}\left(10 \mathrm{~mL}\right.$, sat. aq.) and $\mathrm{Na}_{2} \mathrm{~S}_{2} \mathrm{O}_{3}(10 \mathrm{~mL}$, sat. aq.). The resulting mixture was extracted with EtOAc $(3 \times 50 \mathrm{~mL})$, and the combined organic layers were washed with brine $(10 \mathrm{~mL})$, dried $\left(\mathrm{Na}_{2} \mathrm{SO}_{4}\right)$, and concentrated in vacuo. Flash column chromatography (silica gel, hexanes:EtOAc:Et ${ }_{3} \mathrm{~N} \quad 1: 1: 0.01$ ) afforded aldehyde $71(236 \mathrm{mg}, 83 \%)$ as a colorless oil. 71: $R_{\mathrm{f}}=0.40$ (silica gel, hexanes:EtOAc 3:2); $[\alpha]_{\mathrm{D}}^{25}=+3.6\left(\mathrm{CHCl}_{3}, c=0.9\right)$; IR (film) $v_{\max } 3364,2933,1719,1688,1383,1318,1031$ $\mathrm{cm}^{-1} ;{ }^{1} \mathrm{H}$ NMR $\left(600 \mathrm{MHz}, \mathrm{CDCl}_{3}\right): \delta=9.42(\mathrm{~s}, 1 \mathrm{H}), 6.95(\mathrm{dt}, J=13.8,6.9 \mathrm{~Hz}, 1 \mathrm{H}), 6.33(\mathrm{dd}, J$ $=10.0,1.3 \mathrm{~Hz}, 1 \mathrm{H}), 6.20(\mathrm{dd}, J=15.0,10.8 \mathrm{~Hz}, 1 \mathrm{H}), 5.78(\mathrm{dt}, J=14.1,1.6 \mathrm{~Hz}, 1 \mathrm{H}), 5.76(\mathrm{~d}, J$ $=10.7 \mathrm{~Hz}, 1 \mathrm{H}), 5.73(\mathrm{ddd}, J=17.5,10.5,7.4 \mathrm{~Hz}, 1 \mathrm{H}), 5.67(\mathrm{ddd}, J=17.0,10.5,7.6 \mathrm{~Hz}, 1 \mathrm{H})$, 
$5.55(\mathrm{dt}, J=14.5,6.9 \mathrm{~Hz}, 1 \mathrm{H}), 5.34(\mathrm{~d}, J=8.4 \mathrm{~Hz}, 1 \mathrm{H}), 5.31$ (brs, $1 \mathrm{H}), 5.23(\mathrm{~d}, J=7.3 \mathrm{~Hz}, 1$ H), $5.21(\mathrm{~s}, 1 \mathrm{H}), 5.11$ (ddd, $J=11.7,7.4,5.8 \mathrm{~Hz}, 1 \mathrm{H}), 4.89$ (dt, $J=9.1,4.8 \mathrm{~Hz}, 1 \mathrm{H}), 4.71(\mathrm{~d}, J$ $=3.7 \mathrm{~Hz}, 1 \mathrm{H}), 4.71(\mathrm{~d}, J=3.7 \mathrm{~Hz}, 1 \mathrm{H}), 4.78(\mathrm{brs}, 2 \mathrm{H}), 4.59(\mathrm{~d}, J=6.8 \mathrm{~Hz}, 1 \mathrm{H}), 4.12(\mathrm{dd}, J=$ 7.2, $4.9 \mathrm{~Hz}, 1 \mathrm{H}), 4.01$ (bm, $1 \mathrm{H}), 3.40$ (s, $3 \mathrm{H}), 3.39$ (s, $3 \mathrm{H}), 2.98$ (ddq, $J=11.7,10.7,6.6 \mathrm{~Hz}, 1$ H), 2.30-2.22 (m, 4 H), 2.20-2.14 (m, $2 \mathrm{H}), 1.57(\mathrm{~s}, 3 \mathrm{H}), 1.58(\mathrm{~s}, 3 \mathrm{H}), 1.78-1.68(\mathrm{~m}, 3 \mathrm{H})$, $1.70-1.50(\mathrm{~m}, 4 \mathrm{H}), 1.11 \mathrm{ppm}(\mathrm{d}, J=6.6 \mathrm{~Hz}, 3 \mathrm{H}) ;{ }^{13} \mathrm{C} \mathrm{NMR}\left(150 \mathrm{MHz}, \mathrm{CDCl}_{3}\right): \delta=195.2$, $166.0,156.5,154.5,149.5,139.4,138.1,134.0,132.1,131.5,128.2,126.9,121.2,119.4,117.4$ 94.0, 93.7, 77.8, 76.9, 75.3, 74.2, 55.7, 55.5, 42.4, 37.1, 34.8, 32.1, 30.4, 28.7, 23.8, 16.7, 15.3, 9.5 ppm; HRMS (ESI): calcd for $\mathrm{C}_{33} \mathrm{H}_{51} \mathrm{NO}_{9} \mathrm{Na}^{+}\left[\mathrm{M}+\mathrm{Na}^{+}\right]$628.3456, found 628.3475.

Vinyl iodide 72: To a solution of $\mathrm{CrCl}_{2}$ (456 mg, $3.70 \mathrm{mmol}$, flame-dried) in THF ( $3 \mathrm{~mL}$ ) was added a solution of aldehyde $71(230 \mathrm{mg}, 0.37 \mathrm{mmol})$ and $\mathrm{CHI}_{3}(438 \mathrm{mg}, 1.11 \mathrm{mmol})$ in dioxane

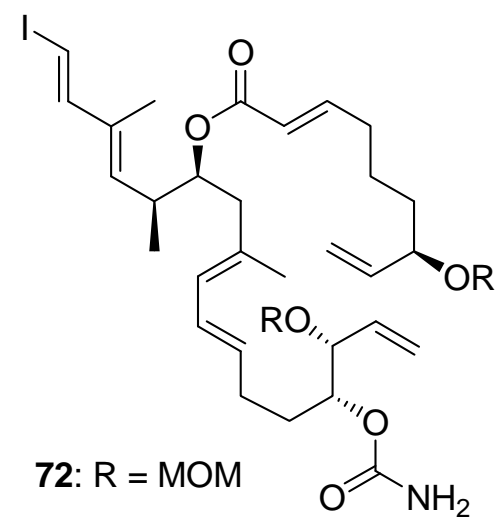
$(18 \mathrm{~mL})$ at room temperature. The reaction mixture was stirred for $2 \mathrm{~h}$ before it was quenched with $\mathrm{NaHCO}_{3}(10 \mathrm{~mL}$, sat. aq.). The resulting mixture was diluted with EtOAc $(50 \mathrm{~mL})$ and filtered through a pad of Celite ${ }^{\circledR}$. The aqueous layer was separated, extracted with EtOAc $(3 \times 20 \mathrm{~mL})$, and the combined organic layers were washed with brine $(20 \mathrm{~mL})$, dried $\left(\mathrm{Na}_{2} \mathrm{SO}_{4}\right)$ and concentrated in vacuo. Flash column chromatography (silica gel, hexanes:EtOAc:Et ${ }_{3} \mathrm{~N}$ 6.5:3.5:0.05) afforded vinyl iodide $72(213 \mathrm{mg}, 77 \%)$ as a white gummy solid. $72: R_{\mathrm{f}}=0.45$ (silica gel, hexanes:EtOAc 3:2); $[\alpha]_{\mathrm{D}}^{25}=-45.4\left(\mathrm{CHCl}_{3}, c=1.8\right)$; IR (film) $v_{\max } 3456,2936,1718$, 1386, 1318, 1097, $1032 \mathrm{~cm}^{-1}$; ${ }^{1} \mathrm{H}$ NMR (600 MHz, $\left.\mathrm{CDCl}_{3}\right): \delta=7.05$ (d, $\left.J=14.6 \mathrm{~Hz}, 1 \mathrm{H}\right), 6.92$ $(\mathrm{dt}, J=13.9,6.9 \mathrm{~Hz}, 1 \mathrm{H}), 6.21(\mathrm{~d}, J=14.6 \mathrm{~Hz}, 1 \mathrm{H}), 6.20(\mathrm{dd}, J=13.9,10.7 \mathrm{~Hz}, 1 \mathrm{H}), 5.79(\mathrm{dt}$, 
$J=15.6 \mathrm{~Hz}, 1 \mathrm{H}), 5.74(\mathrm{~d}, J=11.8 \mathrm{~Hz}, 1 \mathrm{H}), 5.73(\mathrm{ddd}, J=17.7,10.4,7.4 \mathrm{~Hz}, 1 \mathrm{H}), 5.68$ (ddd, $J$ $=18.1,10.5,7.7 \mathrm{~Hz}, 1 \mathrm{H}), 5.53(\mathrm{dt}, J=14.1,6.8 \mathrm{~Hz}, 1 \mathrm{H}), 5.35-5.30(\mathrm{bm}, 2 \mathrm{H}), 5.25-5.19(\mathrm{bm}$, $2 \mathrm{H}), 4.97$ (ddd, $J=10.9,6.8,4.2 \mathrm{~Hz}, 1 \mathrm{H}), 4.89(\mathrm{dt}, J=9.1,4.8 \mathrm{~Hz}, 1 \mathrm{H}), 4.72(\mathrm{~d}, J=4.2 \mathrm{~Hz}, 1$ H), $4.71(\mathrm{~d}, J=4.2 \mathrm{~Hz}, 1 \mathrm{H}), 4.75-4.52(\mathrm{brs}, 2 \mathrm{H}), 4.59$ (d, $J=3.6 \mathrm{~Hz}, 1 \mathrm{H}), 4.55(\mathrm{~d}, J=6.6 \mathrm{~Hz}$, $1 \mathrm{H}), 4.12(\mathrm{dd}, J=7.3,5.0 \mathrm{~Hz}, 1 \mathrm{H}), 4.00(\mathrm{bm}, 1 \mathrm{H}), 3.41(\mathrm{~s}, 3 \mathrm{H}), 3.36(\mathrm{~s}, 3 \mathrm{H}), 2.74$ (ddq, $J=$ 11.8, 10.9, 6.6 Hz, $1 \mathrm{H}), 2.31-2.10(\mathrm{~m}, 6 \mathrm{H}), 1.73(\mathrm{~s}, 3 \mathrm{H}), 1.71(\mathrm{~d}, J=1.2 \mathrm{~Hz}, 3 \mathrm{H}), 1.77-1.50$ (m, $7 \mathrm{H}), 1.00 \mathrm{ppm}(\mathrm{d}, J=6.6 \mathrm{~Hz}, 3 \mathrm{H}) ;{ }^{13} \mathrm{C} \mathrm{NMR}\left(150 \mathrm{MHz}, \mathrm{CDCl}_{3}\right): \delta=166.2,156.5,149.5$, 149.0, 138.1, 135.4, 134.8, 134.0, 132.2, 131.6. 127.8, 127.1, 121.5, 119.4, 117.4, 94.0, 93.7, $77.8,77.0,75.4,75.1,74.2,55.7,55.5,42.4,36.4,34.8,32.0,30.4,28.7,23.8,16.7,16.3,12.3$ ppm; HRMS (ESI): calcd for $\mathrm{C}_{34} \mathrm{H}_{52} \mathrm{INO}_{8} \mathrm{Na}^{+}\left[\mathrm{M}+\mathrm{Na}^{+}\right]$752.2630, found 752.2654.

Diol 73 and cyclic carbonate: To a solution of bis-MOM ether $72(200 \mathrm{mg}, 0.27 \mathrm{mmol})$ in $\mathrm{MeOH}(15 \mathrm{~mL})$ was added TMSCl $(340 \mu \mathrm{L}, 2.68 \mathrm{mmol})$ at $40{ }^{\circ} \mathrm{C}$. The reaction mixture was<smiles>C=CC(O)CCC/C=C/C(=O)O[C@H](C/C(C)=C/C=C\CC[C@@H](OC(N)=O)C(C)/C=C(C)\C=C\I)[C@H](O)C=C</smiles>
stirred for $1 \mathrm{~h}$ before it was cooled to room temperature, quenched with $\mathrm{NaHCO}_{3}(300 \mathrm{mg})$ and concentrated in vacuo. The resulting residue was dissolved in EtOAc $(15 \mathrm{~mL})$, filtered through a pad of Celite ${ }^{\circledR}$, washed with EtOAc $(2 \times 10 \mathrm{~mL})$ and concentrated in vacuo. Flash column chromatography (silica gel, hexanes:EtOAc:Et 3 N 4:6:0.025 $\rightarrow$ 0:10:0.05) afforded diol 73 (127 $\mathrm{mg}, 74 \%)$ as a colorless gummy solid and cyclic carbonate $74(21 \mathrm{mg}, 12 \%)$ as a gummy solid. 73: $R_{\mathrm{f}}=0.36$ (silica gel, hexanes:EtOAc 2:3); $[\alpha]_{\mathrm{D}}{ }^{25}=+27.0\left(\mathrm{CHCl}_{3}, c=0.85\right) ; \mathrm{IR}($ film) $v_{\max } 3365,2931,1709,1651,1390,1318,1181,1059,991 \mathrm{~cm}^{-1} ;{ }^{1} \mathrm{H}$ NMR $\left(600 \mathrm{MHz}, \mathrm{CDCl}_{3}\right): \delta$ $=7.06(\mathrm{~d}, J=14.6 \mathrm{~Hz}, 1 \mathrm{H}), 6.92(\mathrm{ddd}, J=15.6,6.9,6.9 \mathrm{~Hz}, 1 \mathrm{H}), 6.22(\mathrm{~d}, J=14.6 \mathrm{~Hz}, 1 \mathrm{H})$, 
$6.19(\mathrm{dd}, J=15.0,10.8 \mathrm{~Hz}, 1 \mathrm{H}), 5.91-5.84(\mathrm{~m}, 2 \mathrm{H}), 5.79(\mathrm{~d}, J=15.6 \mathrm{~Hz}, 1 \mathrm{H}), 5.73(\mathrm{~d}, J=$ $10.8 \mathrm{~Hz}, 1 \mathrm{H}), 5.51(\mathrm{ddd}, J=14.5,6.9 .6 .9 \mathrm{~Hz}, 1 \mathrm{H}), 5.38(\mathrm{~d} J=17.1 \mathrm{~Hz}, 1 \mathrm{H}), 5.32(\mathrm{~d}, J=12.9$ Hz, $1 \mathrm{H}), 5.27-5.22(\mathrm{~m}, 2 \mathrm{H}), 5.13(\mathrm{~d}, J=10.4 \mathrm{~Hz}, 1 \mathrm{H}), 4.97$ (ddd, $J=10.5,7.0,3.7 \mathrm{~Hz}, 1 \mathrm{H})$, 4.80-4.65 (bm, 2 H), 4.75 (ddd, $J=14.1,9.4,4.8 \mathrm{~Hz}, 1 \mathrm{H}), 4.20-4.10$ (m, $2 \mathrm{H}), 2.78-2.70$ (m, 1 H), 2.30-2.22 (m, $2 \mathrm{H}), 2.20-2.10(\mathrm{~m}, 4 \mathrm{H}), 1.77-1.66(\mathrm{~m}, 2 \mathrm{H}), 1.64-1.47$ (m, $4 \mathrm{H}), 1.73(\mathrm{~s}, 3$ $\mathrm{H}), 1.72(\mathrm{~s}, 3 \mathrm{H}), 1.00(\mathrm{~d}, J=6.8 \mathrm{~Hz}, 3 \mathrm{H}), 1.04-0.96(\mathrm{~m}, 1 \mathrm{H}), 0.90-0.80 \mathrm{ppm}(\mathrm{m}, 1 \mathrm{H}) ;{ }^{13} \mathrm{C}$ $\operatorname{NMR}\left(150 \mathrm{MHz}, \mathrm{CDCl}_{3}\right): \delta=166.2,157.0,149.5,149.0,141.0,136.9,135.3,134.9,132.3$ $131.5,127.8,127.2,121.4,117.0,114.8,76.9,75.0,74.3,74.2,72.8,42.6,36.6,36.3,32.0,30.4$, 28.7, 23.9, 16.7, 16.4, 12.3 ppm; HRMS (ESI): calcd for $\mathrm{C}_{30} \mathrm{H}_{44} \mathrm{INO}_{6} \mathrm{Na}^{+}\left[\mathrm{M}+\mathrm{Na}^{+}\right]$664.2106, found 664.2118 .

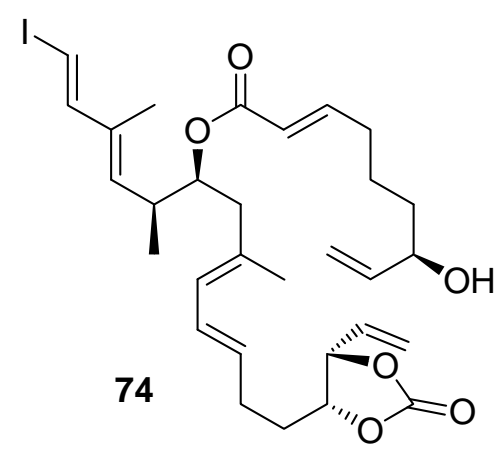

Cyclic carbonate 74: $R_{\mathrm{f}}=0.33$ (silica gel, hexanes:EtOAc 7:3); $[\alpha]_{\mathrm{D}}^{25}=-41.6\left(\mathrm{CHCl}_{3}, c=1.0\right)$; IR (film) $v_{\max } 3493,2932,1803$, 1711, 1432, 1269, 1187, 1048, $988 \mathrm{~cm}^{-1} ;{ }^{1} \mathrm{H}$ NMR $(600 \mathrm{MHz}$, $\left.\mathrm{CDCl}_{3}\right): \delta=7.05(\mathrm{~d}, J=14.0 \mathrm{~Hz}, 1 \mathrm{H}), 6.92(\mathrm{dt}, J=15.6,7.2 \mathrm{~Hz}$, $1 \mathrm{H}), 6.22(\mathrm{~d}, J=9.0 \mathrm{~Hz}, 1 \mathrm{H}), 6.23(\mathrm{~m}, 1 \mathrm{H}), 5.79(\mathrm{td}, J=15.7$, $1.5 \mathrm{~Hz}, 1 \mathrm{H}), 5.74(\mathrm{~d}, J=10.7 \mathrm{~Hz}, 1 \mathrm{H}), 5.50(\mathrm{~m}, 2 \mathrm{H}), 5.44$ (m, $2 \mathrm{H}), 5.31(\mathrm{~d}, J=9.9 \mathrm{~Hz}, 1 \mathrm{H})$, $5.24(\operatorname{td}, J=16.8,1.2 \mathrm{~Hz}, 1 \mathrm{H}), 5.13(\mathrm{td}, J=10.2,1.2 \mathrm{~Hz}, 1 \mathrm{H}), 4.97(\mathrm{ddd}, J=10.8,7.0,3.9 \mathrm{~Hz}$, $1 \mathrm{H}), 4.66(\mathrm{t}, J=7.2 \mathrm{~Hz}, 1 \mathrm{H}), 4.32(\mathrm{dt}, J=8.0,4.6 \mathrm{~Hz}, 1 \mathrm{H}), 4.12(\mathrm{bm}, 1 \mathrm{H}), 2.73(\mathrm{~m}, 1 \mathrm{H})$, 2.35-2.17 (m, $4 \mathrm{H}), 1.91-1.83(\mathrm{~m}, 1 \mathrm{H}), 1.82-1.72(\mathrm{~m}, 2 \mathrm{H}), 1.74(\mathrm{~s}, 3 \mathrm{H}), 1.70(\mathrm{~d}, J=1.1 \mathrm{~Hz}, 3$ H), 1.69 (brs, $2 \mathrm{H}), 1.61-1.49(\mathrm{~m}, 4 \mathrm{H}), 1.02(\mathrm{~m}, 1 \mathrm{H}), 0.99 \mathrm{ppm}(\mathrm{d}, J=6.7 \mathrm{~Hz}, 3 \mathrm{H}) ;{ }^{13} \mathrm{C} \mathrm{NMR}$ $\left(150 \mathrm{MHz}, \mathrm{CDCl}_{3}\right): \delta=166.1,154.2,149.5,149.0,141.0,135.3,134.9,133.4,132.0,129.4$ $128.4,127.2,121.4,121.4,114.9,82.6,81.1,75.0,74.3,72.9,42.5,36.6,36.3,32.8,32.0,28.0$, 
23.8, 16.8, 16.4, 12.3 ppm; HRMS (ESI): calcd for $\mathrm{C}_{30} \mathrm{H}_{41} \mathrm{IO}_{6} \mathrm{Na}^{+}\left[\mathrm{M}+\mathrm{Na}^{+}\right] 647.1840$, found 647.1823.

Macrocycle 75: To a solution of diol $73(96 \mathrm{mg}, 0.148 \mathrm{mmol})$ in $\mathrm{CH}_{2} \mathrm{Cl}_{2}(30 \mathrm{~mL}, 0.005 \mathrm{M})$ was added Grubbs II catalyst $(6.3 \mathrm{mg}, 7.4 \mu \mathrm{mol})$ at room temperature. The reaction mixture was

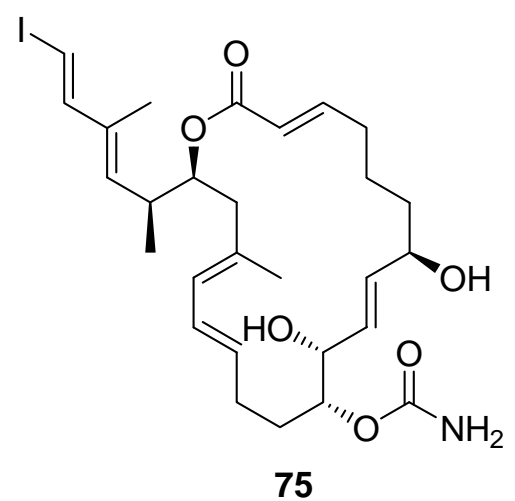
stirred for $1 \mathrm{~h}$ before it was concentrated in vacuo. Flash column chromatography (silica gel, hexanes:EtOAc:Et ${ }_{3} \mathrm{~N}$ 3:7:0.05 $\rightarrow$ EtOAc: $\mathrm{MeOH}: \mathrm{Et}_{3} \mathrm{~N}$ 9.5:0.5:0.05) afforded macrocycle 75 as a colorless gummy solid (73 mg, 80\%). 75: $R_{\mathrm{f}}=0.31$ (silica gel, hexanes:EtOAc 1:7); $[\alpha]_{\mathrm{D}}{ }^{25}=-28.5\left(\mathrm{CHCl}_{3}, c=1.0\right)$ IR (film) $V_{\max } 3355,2924,2854,1714,1392,1316,1219,1076,772 \mathrm{~cm}^{-1}$

${ }^{1} \mathrm{H}$ NMR $\left(600 \mathrm{MHz}, \mathrm{CDCl}_{3}\right) \delta=7.07(\mathrm{~d}, J=14.6 \mathrm{~Hz}, 1 \mathrm{H}), 6.84(\mathrm{ddd}, J=15.2,9.7,5.4 \mathrm{~Hz}, 1 \mathrm{H})$, $6.24(\mathrm{~d}, J=14.6 \mathrm{~Hz}, 1 \mathrm{H}), 6.14(\mathrm{dd}, J=14.9,11.2 \mathrm{~Hz}, 1 \mathrm{H}), 5.79(\mathrm{dd}, J=15.6,7.3 \mathrm{~Hz}, 1 \mathrm{H})$, $5.76(\mathrm{~d}, J=15.4 \mathrm{~Hz}, 1 \mathrm{H}), 5.68(\mathrm{dd}, J=15.6,13.1 \mathrm{~Hz}, 1 \mathrm{H}), 5.67(\mathrm{~d}, J=10.6 \mathrm{~Hz}, 1 \mathrm{H}), 5.48$ (ddd, $J=14.8,9.76,5.0 \mathrm{~Hz}, 1 \mathrm{H}), 5.31(\mathrm{~d}, J=9.6 \mathrm{~Hz}, 1 \mathrm{H}), 4.97(\mathrm{ddd}, J=10.0,6.1,1.9 \mathrm{~Hz}, 1 \mathrm{H}$ ), 4.80-4.72 (brs, 2 H), 4.70 (ddd, $J=10.2,6.3,2.4 \mathrm{~Hz}, 1 \mathrm{H}), 4.31$ (bm, $1 \mathrm{H}), 4.13$ (m, $1 \mathrm{H}), 2.72$ (m, $1 \mathrm{H}), 2.27-2.15(\mathrm{~m}, 2 \mathrm{H}), 2.16-2.06(\mathrm{~m}, 4 \mathrm{H}), 1.76(\mathrm{~s}, 3 \mathrm{H}), 1.71(\mathrm{~s}, 3 \mathrm{H}), 1.67-1.58(\mathrm{~m}, 3$ $\mathrm{H}), 1.54-1.44(\mathrm{~m}, 5 \mathrm{H}), 0.99 \mathrm{ppm}(\mathrm{d}, J=6.7 \mathrm{~Hz}, 3 \mathrm{H}) ;{ }^{13} \mathrm{C} \mathrm{NMR}\left(150 \mathrm{MHz}, \mathrm{CDCl}_{3}\right): \delta=166.3$, $156.9,149.5,148.8,135.2,135.0,134.2,132.0,131.2,129.6,128.0,127.2,121.3,74.4,74.0$ 73.0, 72.3, 45.8, 43.7, 37.4, 36.8, 32.7, 30.3, 28.9, 24.7, 16.9, 16.4, 12.4 ppm; HRMS (ESI): calcd for $\mathrm{C}_{28} \mathrm{H}_{40} \mathrm{INO}_{6} \mathrm{Na}^{+}\left[\mathrm{M}+\mathrm{Na}^{+}\right]$636.1793, found 636.1810 . 
Palmerolide A isomer 19-epi-20-epi-1 and decarbamated palmerolide $\mathbf{A}$ isomer 76: To a mixture of vinyl iodide 75 ( $25 \mathrm{mg}, 40 \mu \mathrm{mol})$, amide 42 ( $8.0 \mathrm{mg}, 81.5 \mu \mathrm{mol}), \mathrm{K}_{2} \mathrm{CO}_{3}(34 \mathrm{mg}, 240$

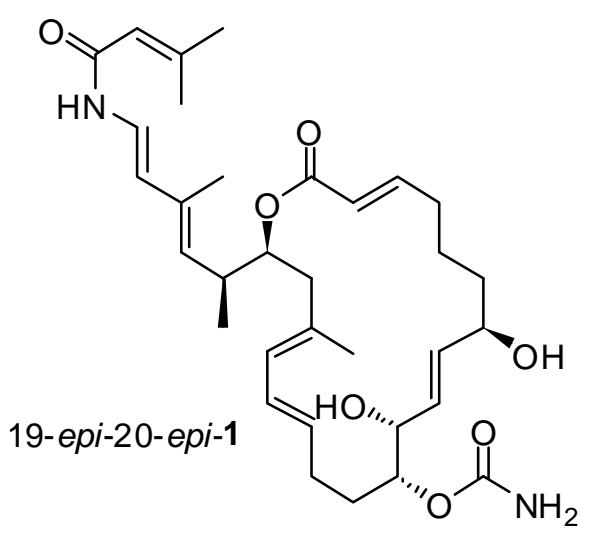
$\mu \mathrm{mol})$ and $\mathrm{CuI}(11.6 \mathrm{mg}, 61 \mu \mathrm{mol})$ in DMF $(2.0 \mathrm{~mL})$ was added $N, N^{\prime}$-dimethylethylenediamine $(14 \mu \mathrm{L}, 122 \mu \mathrm{mol})$ at room temperature. The reaction mixture was stirred for $1 \mathrm{~h}$ before it was concentrated in vacuo. The resulting residue was diluted with EtOAc $(10 \mathrm{~mL})$, filtered through a pad of Celite $^{\circledR}$ and washed with EtOAc $(10 \mathrm{~mL})$, and the filterate was concentrated in vacuo. Preparative TLC purification (silica gel, EtOAc:Et $\mathrm{N}_{3} \mathrm{~N}$ 100:1) afforded 19-epi-20-epi-1 (13.4 mg, 55\%) as a white solid and decarbamated product $76(1.0 \mathrm{mg}, \sim 5 \%)$ as a gummy solid. Palmerolide A isomer 19-epi-20-epi-1: $R_{\mathrm{f}}=0.23$ (silica gel, hexanes:EtOAc 0:1); $[\alpha]_{\mathrm{D}}^{25}=+91.0(\mathrm{MeOH}, c=0.74)$; IR (film) $v_{\max } 3390,2919,2850,1712,1640,1456,1377,1259$, $1145 \mathrm{~cm}^{-1} ;{ }^{1} \mathrm{H}$ NMR $\left(600 \mathrm{MHz}, \mathrm{DMSO}-d^{6}\right): \delta=9.85(\mathrm{~d}, J=10.4 \mathrm{~Hz}, 1 \mathrm{H}), 6.85(\mathrm{dd}, J=14.5$, $10.4 \mathrm{~Hz}, 1 \mathrm{H}), 6.71$ (ddd, $J=15.2,10.1,5.0 \mathrm{~Hz}, 1 \mathrm{H}), 6.05(\mathrm{dd}, J=15.1,10.8 \mathrm{~Hz}, 1 \mathrm{H}), 5.84$ (d, $J=14.5 \mathrm{~Hz}, 1 \mathrm{H}), 5.77$ (d, $J=15.7 \mathrm{~Hz}, 1 \mathrm{H}), 5.69$ (brs, $1 \mathrm{H}), 5.60(\mathrm{~d}, J=10.3 \mathrm{~Hz}, 1 \mathrm{H}), 5.55(\mathrm{dd}$, $J=15.5,8.2 \mathrm{~Hz}, 1 \mathrm{H}), 5.47$ (dd, $J=15.5,2.8 \mathrm{~Hz}, 1 \mathrm{H}), 5.41$ (ddd, $J=14.8,9.6,4.8 \mathrm{~Hz}, 1 \mathrm{H})$, $5.18(\mathrm{~d}, J=4.9 \mathrm{~Hz}, 1 \mathrm{H}), 5.13(\mathrm{~d}, J=9.6 \mathrm{~Hz}, 1 \mathrm{H}), 4.84(\mathrm{ddd}, J=10.0,8.0,1.6 \mathrm{~Hz}, 1 \mathrm{H}), 4.69(\mathrm{~d}$, $J=2.8 \mathrm{~Hz}, 1 \mathrm{H}), 4.49$ (ddd, $J=10.8,4.8,1.6 \mathrm{~Hz}, 1 \mathrm{H}), 4.14$ (brs, $1 \mathrm{H}), 3.82(\mathrm{~m}, 1 \mathrm{H}), 2.69$ (m, 1 H), $2.12(\mathrm{~s}, 3 \mathrm{H}), 2.18-1.89(\mathrm{~m}, 6 \mathrm{H}), 1.83(\mathrm{~s}, 3 \mathrm{H}), 1.71(\mathrm{~s}, 3 \mathrm{H}), 1.61(\mathrm{~s}, 3 \mathrm{H}), 1.59$ (m, $1 \mathrm{H})$, $1.47(\mathrm{~m}, 1 \mathrm{H}), 1.30(\mathrm{~m}, 2 \mathrm{H}), 1.04(\mathrm{~m}, 1 \mathrm{H}), 0.97(\mathrm{~m}, 1 \mathrm{H}), 0.89 \mathrm{ppm}(\mathrm{d}, J=6.8 \mathrm{~Hz}, 3 \mathrm{H}) ;{ }^{13} \mathrm{C}$ NMR (150 MHz, $\left.\mathrm{CDCl}_{3}\right): \delta=166.1,121.3,163.9,157.3,152.5,150.0,134.3,133.3,132.7$, $132.3,130.5,129.6,128.4,127.1,122.9,118.8,117.2,75.8,74.5,73.2,69.9,43.9,38.5,37.3$, 
32.6, 30.1, 30.1, 27.7, 25.7, 20.3, 17.7, 16.9, 13.3 ppm; HRMS (ESI): calcd for $\mathrm{C}_{28} \mathrm{H}_{40} \mathrm{INO}_{6} \mathrm{Na}^{+}$ $\left[\mathrm{M}+\mathrm{Na}^{+}\right]$607.3354, found 607.3367.

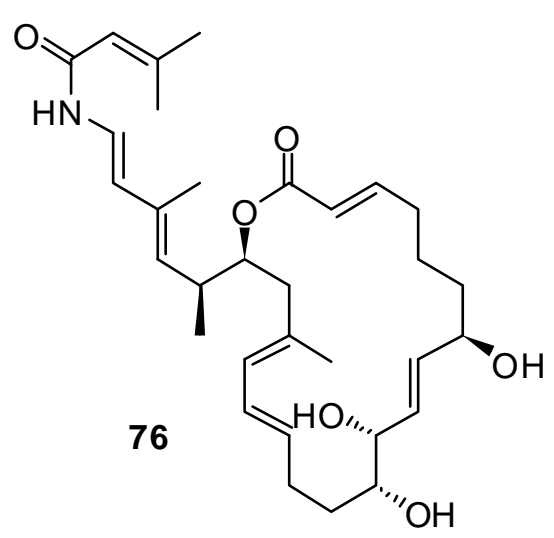

Decarbamated palmerolide A isomer 76: $R_{\mathrm{f}}=0.32$ (silica gel, hexanes:EtOAc 0:1); $[\alpha]_{\mathrm{D}}^{25}=+65.2(\mathrm{MeOH}, c=0.39)$; IR (film) $v_{\max } 3330,2928,1695,1638,1522,1262,1194,1018$ $\mathrm{cm}^{-1} ;{ }^{1} \mathrm{H}$ NMR $\left(600 \mathrm{MHz}, \mathrm{DMSO}-d^{6}\right): \delta=9.85(\mathrm{~d}, J=10.8$ $\mathrm{Hz}, 1 \mathrm{H}), 6.85$ (dd, $J=15.0,10.2 \mathrm{~Hz}, 1 \mathrm{H}), 6.72$ (ddd, $J=$ 15.0, 9.6, $4.8 \mathrm{~Hz}, 1 \mathrm{H}), 6.05$ (dd, $J=14.4,5.4 \mathrm{~Hz}, 1 \mathrm{H}), 5.85$

$(\mathrm{d}, J=14.4 \mathrm{~Hz}, 1 \mathrm{H}), 5.76(\mathrm{~d}, J=15.6 \mathrm{~Hz}, 1 \mathrm{H}), 5.69(\mathrm{~s}, 1 \mathrm{H}), 5.60(\mathrm{~d}, J=11.4 \mathrm{~Hz}, 1 \mathrm{H}), 5.57$ $(\mathrm{dd}, J=15.6,3.0 \mathrm{~Hz}, 1 \mathrm{H}), 5.49(\mathrm{ddd}, J=15.6,7.8,1.8 \mathrm{~Hz}, 1 \mathrm{H}), 5.42(\mathrm{ddd}, J=13.8,10.2,4.2$ $\mathrm{Hz}, 1 \mathrm{H}), 5.14(\mathrm{~d}, J=9.6 \mathrm{~Hz}, 1 \mathrm{H}), 4.85(\mathrm{ddd}, J=10.8,7.2,1.8 \mathrm{~Hz}, 1 \mathrm{H}), 4.78(\mathrm{~d}, J=4.2 \mathrm{~Hz}, 1$ H), $4.68(\mathrm{~d}, J=4.8 \mathrm{~Hz}, 1 \mathrm{H}), 4.60(\mathrm{~d}, J=3.6 \mathrm{~Hz}, 1 \mathrm{H}), 3.97$ (bs, $1 \mathrm{H}), 3.82(\mathrm{bs}, 1 \mathrm{H}), 3.09$ (m, 1 H), 2.69 (m, 1 H), 2.12 (s, 3 H), 2.20-2.00 (m, 4 H), 1.90 (m, 2 H), 1.83 (s, 3 H), $1.71(\mathrm{~s}, 3 \mathrm{H})$, $1.61(\mathrm{~s}, 3 \mathrm{H}), 1.52-1.40(\mathrm{~m}, 2 \mathrm{H}), 1.36-1.20(\mathrm{~m}, 2 \mathrm{H}), 1.09$ (m, $1 \mathrm{H}), 0.90(\mathrm{~d}, J=6.6 \mathrm{~Hz}, 3 \mathrm{H})$, 0.85 ppm (m, $1 \mathrm{H}) ;{ }^{13} \mathrm{C}$ NMR (150 MHz, DMSO- $\left.d^{6}\right): \delta=166.2,149.5,148.8,135.2,135.1$, 134.7, 132.0, 131.3, 129.8, 127.8, 127.4, 121.4, 75.5, 74.3, 74.0, 74.0, 72.2, 43.3, 37.3, 36.5, 32.5, 28.4, 24.2, 16.9, 16.4, 12.4, 8.7 ppm; HRMS (ESI): calcd for $\mathrm{C}_{32} \mathrm{H}_{47} \mathrm{NO}_{6} \mathrm{Na}^{+}\left[\mathrm{M}+\mathrm{Na}^{+}\right]$ 564.3296, found 564.3284

Macrocycle 77: To a solution of cyclic carbonate 74 (13.0 mg, $20.9 \mu \mathrm{mol})$ in $\mathrm{CH}_{2} \mathrm{Cl}_{2}(4.8 \mathrm{~mL}$, $0.005 \mathrm{M})$ was added Grubbs II catalyst $(3.6 \mathrm{mg}, 4.18 \mu \mathrm{mol})$ at room tempertature. The reaction mixture was stirred for $4 \mathrm{~h}$ before it was concentrated in vacuo. Preparative TLC purification (silica gel, hexanes:EtOAc 1:1) afforded macrocycle $77(9.5 \mathrm{mg}, 76 \%)$ as a colorless gummy 


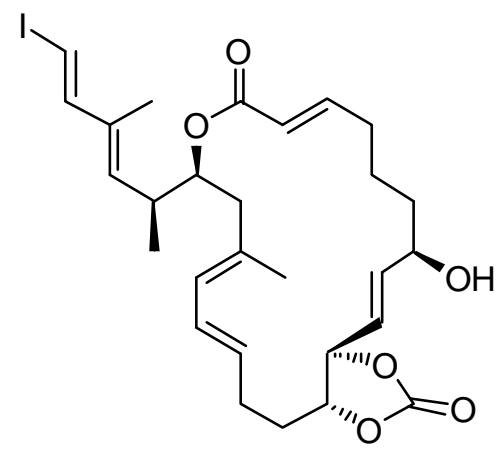

77

solid. 77: $R_{\mathrm{f}}=0.30$ (silica gel, hexanes:EtOAc 7:3); $[\alpha]_{\mathrm{D}}{ }^{25}=$ $-53.0\left(\mathrm{CHCl}_{3}, c=0.89\right)$; IR (film) $v_{\max } 3377,2927,1801,1712$, $1442,1271,1178,1049,773 \mathrm{~cm}^{-1} ;{ }^{1} \mathrm{H}$ NMR $\left(600 \mathrm{MHz}, \mathrm{CDCl}_{3}\right)$ : $\delta=7.07(\mathrm{~d}, J=14.6 \mathrm{~Hz}, 1 \mathrm{H}), 6.84(\mathrm{dt}, J=15.6,6.9 \mathrm{~Hz}, 1 \mathrm{H})$, $6.23(\mathrm{~d}, J=14.6 \mathrm{~Hz}, 1 \mathrm{H}), 6.24(\mathrm{~m}, 2 \mathrm{H}), 5.95(\mathrm{dd}, J=15.5,3.6$ $\mathrm{Hz}, 1 \mathrm{H}), 5.83$ (ddd, $J=15.5,8.0,1.7 \mathrm{~Hz}, 1 \mathrm{H}), 5.74(\mathrm{dt}, J=15.6$,

$1.4 \mathrm{~Hz}, 1 \mathrm{H}), 5.31(\mathrm{~d}, J=10.9 \mathrm{~Hz}, 1 \mathrm{H}), 5.46(\mathrm{dt}, J=15.0,7.5 \mathrm{~Hz}, 1 \mathrm{H}), 5.31(\mathrm{~d}, J=10.0 \mathrm{~Hz}, 1$ H), $5.00(\mathrm{ddd}, J=11.0,7.6,2.1 \mathrm{~Hz}, 1 \mathrm{H}), 4.68(\mathrm{~m}, 1 \mathrm{H}), 4.41-4.35(\mathrm{~m}, 2 \mathrm{H}), 2.77-2.72(\mathrm{~m}, 1 \mathrm{H})$, 2.39-2.30 (m, $1 \mathrm{H}), 2.30-2.10(\mathrm{~m}, 6 \mathrm{H}), 2.03-1.96(\mathrm{~m}, 1 \mathrm{H}), 1.75$ (d, $J=1.2 \mathrm{~Hz}, 3 \mathrm{H}), 1.72(\mathrm{~s}, 3$ $\mathrm{H}), 1.70-1.40(\mathrm{~m}, 4 \mathrm{H}), 1.00 \mathrm{ppm}(\mathrm{d}, J=6.6 \mathrm{~Hz}, 3 \mathrm{H}) ;{ }^{13} \mathrm{C} \mathrm{NMR}\left(150 \mathrm{MHz}, \mathrm{CDCl}_{3}\right): \delta=166.0$, $154.2,149.5,148.2,140.3,135.2,135.0,133.4,129.3,128.0,127.3,123.3,121.8,82.4,80.2$ 74.4, 74.0, 70.2, 43.0, 37.3, 35.4, 33.4, 32.3, 27.2, 22.8, 16.9, 16.6, 12.4 ppm; HRMS (ESI): calcd for $\mathrm{C}_{28} \mathrm{H}_{37} \mathrm{IO}_{6} \mathrm{Na}^{+}\left[\mathrm{M}+\mathrm{Na}^{+}\right]$619.1527, found 619.1519 .

Triol 78: A solution of cyclic carbonate $77(9.5 \mathrm{mg}, 15.9 \mu \mathrm{mol})$ in a mixture of $\mathrm{Et}_{3} \mathrm{~N} / \mathrm{MeOH} / \mathrm{H}_{2} \mathrm{O}(1: 5: 1,2.0 \mathrm{~mL})$ was heated at $40{ }^{\circ} \mathrm{C}$ for $6 \mathrm{~h}$ before it was cooled to room

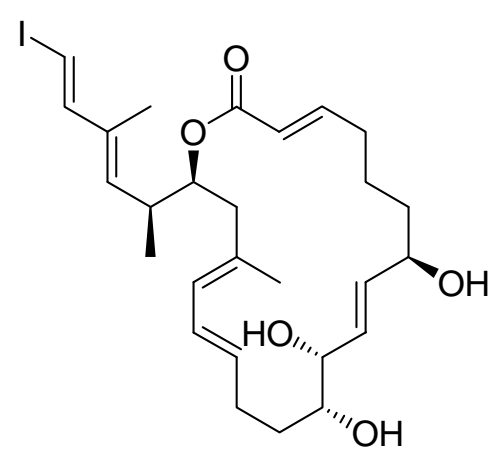

78 temperature and concentrated in vacuo. Preparative TLC purification (silica gel, hexanes:EtOAc 1:9) afforded triol 78 (5.6 $\mathrm{mg}, 62 \%$ ) as a gummy solid. 78: $R_{\mathrm{f}}=0.46$ (silica gel, hexanes:EtOAc 0:1); $[\alpha]_{\mathrm{D}}^{25}=+4.7\left(\mathrm{CHCl}_{3}, c=0.84\right)$; IR (film) $V_{\max } 3361,2927,1715,1654,1261,1185,1016,754 \mathrm{~cm}^{-1} ;{ }^{1} \mathrm{H}$ $\operatorname{NMR}\left(600 \mathrm{MHz}, \mathrm{CDCl}_{3}\right): \delta=7.06(\mathrm{~d}, J=14.6 \mathrm{~Hz}, 1 \mathrm{H}), 6.85$ (ddd, $J=14.9,8.9,5.5 \mathrm{~Hz}, 1 \mathrm{H}), 6.23(\mathrm{~d}, J=5.9 \mathrm{~Hz}, 1 \mathrm{H}), 6.17(\mathrm{dd}, J=14.6,10.7 \mathrm{~Hz}, 1 \mathrm{H}), 5.78$ 
(bm, $1 \mathrm{H}), 5.68(\mathrm{~d}, J=10.4 \mathrm{~Hz}, 1 \mathrm{H}), 5.53(\mathrm{ddd}, J=14.6,9.2,4.9 \mathrm{~Hz}, 1 \mathrm{H}), 5.31(\mathrm{~d}, J=9.9 \mathrm{~Hz}$, $1 \mathrm{H}), 5.00$ (dt, $J=9.9,1.7 \mathrm{~Hz}, 1 \mathrm{H}$ ), 4.15 (brd, $1 \mathrm{H}), 3.99$ (brs, $1 \mathrm{H}$ ), 3.50 (brs, $1 \mathrm{H}$ ), 3.14 (brd, $J$ $=3.6 \mathrm{~Hz}, 1 \mathrm{H}), 2.72(\mathrm{~m}, 1 \mathrm{H}), 2.35-2.05(\mathrm{~m}, 8 \mathrm{H}), 1.75(\mathrm{~s}, 3 \mathrm{H}), 1.71(\mathrm{~s}, 3 \mathrm{H}), 1.54-1.04(\mathrm{~m}, 8$ H), $0.99 \mathrm{ppm}(\mathrm{d}, J=7.2 \mathrm{~Hz}, 3 \mathrm{H}) ;{ }^{13} \mathrm{C} \mathrm{NMR}\left(150 \mathrm{MHz}, \mathrm{CDCl}_{3}\right): \delta=166.2,149.5,148.8,135.2$, 135.1, 134.7, 132.0, 131.3, 129.8, 127.8, 127.4, 121.4, 74.3, 74.0, 74.0, 72.2, 46.2, 43.34, 37.3, 36.5, 32.5, 28.4, 24.2, 16.9, 16.4, 12.4, 8.7 ppm; HRMS (ESI): calcd for $\mathrm{C}_{27} \mathrm{H}_{39} \mathrm{IO}_{5} \mathrm{Na}^{+}\left[\mathrm{M}+\mathrm{Na}^{+}\right]$ 593.1734, found 593.1722.

Enamide 76: To a mixture of vinyl iodide 75 (10 mg, $17.5 \mu \mathrm{mol})$, amide 42 ( $3.5 \mathrm{mg}, 35 \mu \mathrm{mol})$, $\mathrm{K}_{2} \mathrm{CO}_{3}(12.2 \mathrm{mg}, 87 \mu \mathrm{mol})$ and $\mathrm{CuI}(5.0 \mathrm{mg}, 26 \mu \mathrm{mol})$ in DMF $(2.0 \mathrm{~mL})$ was added $N, N^{\prime}-$

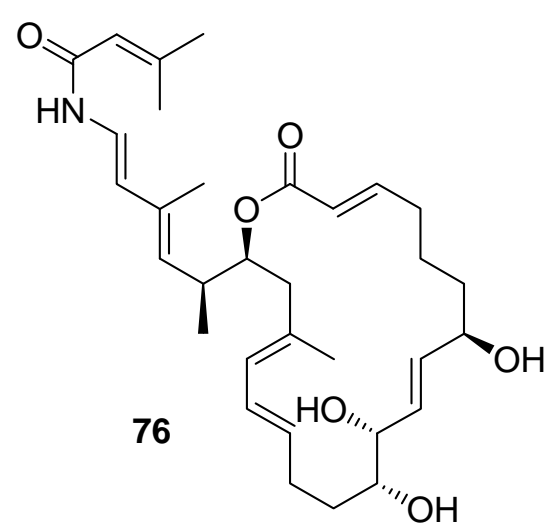
dimethylethylenediamine $(5.8 \mu \mathrm{L}, \quad 53 \mu \mathrm{mol})$ at room temperature. The reaction mixture was stirred for $1 \mathrm{~h}$ before it was concentrated in vacuo. The resulting residue was diluted with EtOAc $(10 \mathrm{~mL})$, filtered through a pad of Celite ${ }^{\circledR}$ and washed with EtOAc $(10 \mathrm{~mL})$, and concentrated in vacuo. Preparative TLC purification (silica gel, EtOAc:Et ${ }_{3} \mathrm{~N}$ 100:1) afforded enamide $76(5.1 \mathrm{mg}, 54 \%)$ as a colorless oil. All physical properties of this compound were identical to those reported for decarbamated product $\mathbf{7 6}$ obtained from vinyl iodide $\mathbf{7 5 .}$

Tetraene 79: To a mixture of vinyl stannane ent-3 (317 mg, $0.612 \mathrm{mmol})$, vinyl iodide 2 (200 $\mathrm{mg}, 0.472 \mathrm{mmol}), \mathrm{Pd}(\mathrm{dba})_{2}(80 \mathrm{mg}, 0.145 \mathrm{mmol}), \mathrm{AsPh}_{3}(289 \mathrm{mg}, 0.944 \mathrm{mmol})$ and $\mathrm{LiCl}(58 \mathrm{mg}$, $1.45 \mathrm{mmol}$, freshly dried by heating with a heatgun for 20 min under vacuum) was added NMP (5.0 $\mathrm{mL}$, degassed by using the freeze-thaw process sequence three times) at room temperature. 


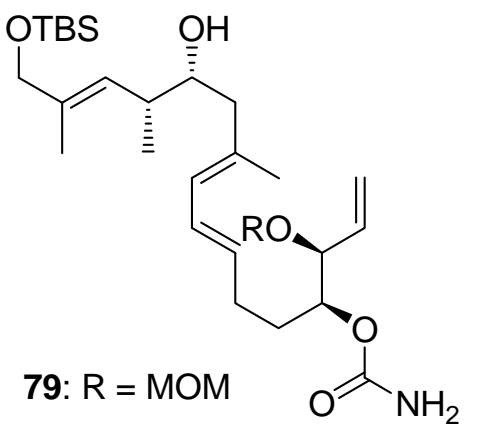

The reaction mixture was stirred for $12 \mathrm{~h}$ before it was quenched with water $(10 \mathrm{~mL})$. The resulting mixture was extracted with EtOAc $(3 \times 30 \mathrm{~mL})$, and the combined organic layers were dried $\left(\mathrm{Na}_{2} \mathrm{SO}_{4}\right)$ and concentrated in vacuo. Flash column chromatography (silica gel, hexanes:EtOAc:Et $3 \mathrm{~N} \quad 3: 2: 0.01$ ) afforded tetraene $79(138 \mathrm{mg}, 56 \%)$ as a pale yellow oil. 79: $R_{\mathrm{f}}=0.30$ (silica gel, hexanes:EtOAc 3:2); $[\alpha]_{\mathrm{D}}^{25}=+36.0\left(\mathrm{CH}_{2} \mathrm{Cl}_{2}, c=0.25\right)$; IR (film) $v_{\max } 3356,2955,2929,2857,1737,1603,1442$, 1389, 1257, 1032, $837 \mathrm{~cm}^{-1} ;{ }^{1} \mathrm{H}$ NMR (600 MHz, $\left.\mathrm{CDCl}_{3}\right): \delta=6.26(\mathrm{dd}, J=15.0,10.8 \mathrm{~Hz}, 1 \mathrm{H})$, $5.86(\mathrm{~d}, J=10.8 \mathrm{~Hz}, 1 \mathrm{H}), 5.72(\mathrm{ddd}, J=17.4,10.2,6.6 \mathrm{~Hz}, 1 \mathrm{H}), 5.59(\mathrm{dt}, J=15.0,6.6 \mathrm{~Hz}, 1 \mathrm{H})$, $5.32(\mathrm{brd}, J=9.6 \mathrm{~Hz}, 1 \mathrm{H}), 5.31(\mathrm{brd}, J=17.4 \mathrm{~Hz}, 1 \mathrm{H}), 5.26(\mathrm{~d}, J=9.6 \mathrm{~Hz}, 1 \mathrm{H}), 4.89(\mathrm{dt}, J=$ 7.8, $4.8 \mathrm{~Hz}, 1 \mathrm{H}), 4.84$ (brs, $2 \mathrm{H}), 4.70(\mathrm{~d}, J=6.6 \mathrm{~Hz}, 1 \mathrm{H}), 4.58$ (d, $J=7.2 \mathrm{~Hz}, 1 \mathrm{H}), 4.12$ (dd, $J$ $=10.8,6.0 \mathrm{~Hz}, 1 \mathrm{H}), 4.02(\mathrm{~s}, 2 \mathrm{H}), 3.50(\mathrm{ddd}, J=10.2,6.0,3.0 \mathrm{~Hz}, 1 \mathrm{H}), 3.39(\mathrm{~s}, 3 \mathrm{H}), 2.46(\mathrm{ddq}$, $J=9.6,7.2,6.6 \mathrm{~Hz}, 1 \mathrm{H}), 2.31(\mathrm{~d}, J=13.2 \mathrm{~Hz}, 1 \mathrm{H}), 2.21-2.14(\mathrm{~m}, 2 \mathrm{H}), 1.94(\mathrm{dd}, J=13.8,10.2$ Hz, 1 H), 1.87 (s, 1 H), 1.79-1.69 (m, 2 H), 1.76 (s, 3 H), 1.67 (s, 3 H), 1.04 (d, J = 6.6 Hz, 3 H), 0.91 (s, $9 \mathrm{H}), 0.07 \mathrm{ppm}(\mathrm{s}, 6 \mathrm{H}) ;{ }^{13} \mathrm{C} \mathrm{NMR}\left(150 \mathrm{MHz}, \mathrm{CDCl}_{3}\right): \delta=156.6,135.0,134.0,133.6$ $131.9,127.7,126.9,126.6,119.4,94.0,77.9,75.3,73.0,68.4,55.7,45.6,38.3,30.5,28.8,25.9$, 18.4, 16.7, 16.5, 13.8, -5.2 ppm; HRMS (ESI): calcd for $\mathrm{C}_{28} \mathrm{H}_{51} \mathrm{NO}_{6} \mathrm{SiNa}^{+}\left[\mathrm{M}+\mathrm{Na}^{+}\right]$548.3378, found 548.3368 .

Ester 80: To a solution of acid ent-4 $(84 \mathrm{mg}, 0.395 \mathrm{mmol}), \mathrm{Et}_{3} \mathrm{~N}(0.182 \mathrm{~mL}, 1.30 \mathrm{mmol})$ in toluene $(1.0 \mathrm{~mL})$ was added 2,4,6-trichlorobenzoyl chloride $(62 \mu \mathrm{L}, 0.395 \mathrm{mmol})$ at room temperature. The reaction mixture was stirred for $1 \mathrm{~h}$ before a solution of alcohol 79 (138 $\mathrm{mg}$, $0.263 \mathrm{mmol})$ and 4-DMAP $(32 \mathrm{mg}, 0.263 \mathrm{mmol})$ in toluene $(1.5 \mathrm{~mL})$ was added at room 


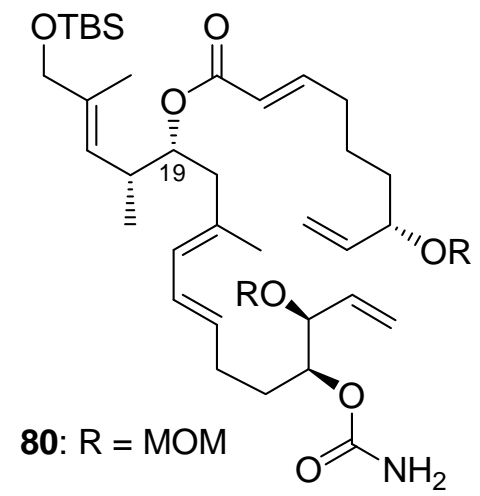

temperature. The reaction mixture was stirred for $12 \mathrm{~h}$ before it was quenched with $\mathrm{NH}_{4} \mathrm{Cl}(15 \mathrm{~mL}$, sat. aq.). The resulting mixture was extracted with EtOAc $(3 \times 30 \mathrm{~mL})$, and the combined organic layers were dried $\left(\mathrm{Na}_{2} \mathrm{SO}_{4}\right)$ and concentrated in vacuo. Flash column chromatography (silica gel, hexanes:EtOAc: $\mathrm{Et}_{3} \mathrm{~N}$ 4:2:0.01) afforded ester $80(110 \mathrm{mg}, 58 \%)$ as a colorless oil. 80: $R_{\mathrm{f}}=0.35$

(silica gel, hexanes:EtOAc 3:2); $[\alpha]_{\mathrm{D}}{ }^{25}=-2.8\left(\mathrm{CH}_{2} \mathrm{Cl}_{2}, c=0.18\right)$; IR (film) $v_{\max } 3356,2930$, $2857,1719,1443,1385,1255,1098 \mathrm{~cm}^{-1} ;{ }^{1} \mathrm{H}$ NMR $\left(600 \mathrm{MHz}, \mathrm{CDCl}_{3}\right): \delta=6.90(\mathrm{dt}, J=15.6$, $6.9 \mathrm{~Hz}, 1 \mathrm{H}), 6.19(\mathrm{dd}, J=15.0,10.8 \mathrm{~Hz}, 1 \mathrm{H}), 5.78(\mathrm{~d}, J=15.6 \mathrm{~Hz}, 1 \mathrm{H}), 5.74(\mathrm{~d}, J=10.2 \mathrm{~Hz}, 1$ H), $5.72(\mathrm{ddd}, J=17.4,10.2,7.2 \mathrm{~Hz}, 1 \mathrm{H}), 5.68(\mathrm{ddd}, J=17.4,10.2,3.0 \mathrm{~Hz}, 1 \mathrm{H}), 5.50(\mathrm{dt}, J=$ 15.0, 7.2 Hz, $1 \mathrm{H}), 5.32$ (brd, $J=10.2 \mathrm{~Hz}, 1 \mathrm{H}), 5.31$ (brd, $J=17.4 \mathrm{~Hz}, 1 \mathrm{H}), 5.25$ (d, $J=10.2$ $\mathrm{Hz}, 1 \mathrm{H}$ ), 5.21 (brd, $J=9.6 \mathrm{~Hz}, 1 \mathrm{H}$ ), 5.20 (brd, $J=17.4 \mathrm{~Hz}, 1 \mathrm{H}$ ), 4.95 (ddd, $J=9.0,7.2,4.2 \mathrm{~Hz}$, $1 \mathrm{H}), 4.88(\mathrm{dt}, J=9.0,4.6 \mathrm{~Hz}, 1 \mathrm{H}), 4.77$ (brs, $2 \mathrm{H}), 4.71(\mathrm{~d}, J=6.6 \mathrm{~Hz}, 1 \mathrm{H}), 4.70(\mathrm{~d}, J=7.2 \mathrm{~Hz}$, $1 \mathrm{H}), 4.58(\mathrm{~d}, J=6.6 \mathrm{~Hz}, 1 \mathrm{H}), 4.53(\mathrm{~d}, J=7.2 \mathrm{~Hz}, 1 \mathrm{H}), 4.11(\mathrm{dd}, J=6.6,4.8 \mathrm{~Hz}, 1 \mathrm{H}), 4.00(\mathrm{~s}$, $2 \mathrm{H}), 3.99$ (q, $J=7.2 \mathrm{~Hz}, 1 \mathrm{H}), 3.39$ (s, $3 \mathrm{H}), 3.37$ (s, $3 \mathrm{H}), 2.64$ (ddq, $J=9.6,7.2,6.6 \mathrm{~Hz}, 1 \mathrm{H})$, $2.30(\mathrm{dd}, J=13.8,3.6 \mathrm{~Hz}, 1 \mathrm{H}), 2.23-2.13(\mathrm{~m}, 5 \mathrm{H}), 1.77-1.48(\mathrm{~m}, 6 \mathrm{H}), 1.72(\mathrm{~s}, 3 \mathrm{H}), 1.58(\mathrm{~s}, 3$ H), $0.96(\mathrm{~d}, J=6.6 \mathrm{~Hz}, 3 \mathrm{H}), 0.93(\mathrm{~s}, 9 \mathrm{H}), 0.06 \mathrm{ppm}(\mathrm{s}, 6 \mathrm{H}) ;{ }^{13} \mathrm{C} \mathrm{NMR}\left(150 \mathrm{MHz}, \mathrm{CDCl}_{3}\right): \delta=$ $166.3,156.5,148.6,138.1,135.3,134.0,132.7,131.3,127.5,127.2,125.6,121.6,119.4,117.4$, $94.0,93.7,77.8,77.0,75.7,75.4,68.2,55.7,55.4,42.5,36.0,34.8,32.0,30.4,28.7,25.9,23.8$, 18.4, 16.7, 16.6, 13.8, -5.2, -5.2 ppm; HRMS (ESI): calcd for $\mathrm{C}_{39} \mathrm{H}_{67} \mathrm{NO}_{9} \mathrm{SiNa}^{+}\left[\mathrm{M}+\mathrm{Na}^{+}\right]$ 744.4477, found 744.4463 . 
Ester 81: To a solution of acid ent-4 $(485 \mathrm{mg}, 2.26 \mathrm{mmol})$ in toluene $(15 \mathrm{~mL})$ was added $\mathrm{Et}_{3} \mathrm{~N}$ (0.39 $\mathrm{mL}, 2.83 \mathrm{mmol})$ followed by 2,4,6-trichlorobenzoyl chloride $(0.35 \mathrm{~mL}, 2.26 \mathrm{mmol})$ at

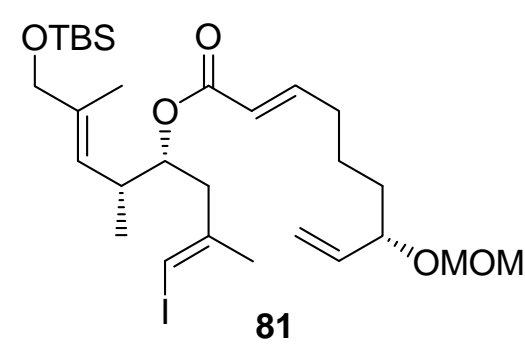
room temperature. The reaction mixture was stirred for $1 \mathrm{~h}$ before a solution of vinyl iodide $2(800 \mathrm{mg}, 1.89 \mathrm{mmol})$ and 4DMAP $(230 \mathrm{mg}, 1.89 \mathrm{mmol})$ in toluene $(10 \mathrm{~mL})$ was added at room temperature. The reaction mixture was stirred for $12 \mathrm{~h}$ before it was diluted with $\mathrm{H}_{2} \mathrm{O}(20 \mathrm{~mL})$ and EtOAc $(50 \mathrm{~mL})$. The resulting mixture was extracted with EtOAc $(2 \times 50 \mathrm{~mL})$, and the combined organic layers were washed with brine $(20 \mathrm{~mL})$, dried $\left(\mathrm{Na}_{2} \mathrm{SO}_{4}\right)$ and concentrated in vacuo. Flash column chromatography (silica gel, hexanes:EtOAc:Et ${ }_{3} \mathrm{~N}$ 4:1:0.05) afforded ester $\mathbf{8 1}(985 \mathrm{mg}, 84 \%)$ as a colorless oil. 81: $R_{\mathrm{f}}=0.65$ (silica gel, hexanes:EtOAc 9:1); $[\alpha]_{\mathrm{D}}^{25}=-4.2\left(\mathrm{CHCl}_{3}, c=2.0\right)$; IR (film) $v_{\max } 2929,1720,1653$, $1462,1257,1149,1098,1033,837 \mathrm{~cm}^{-1} ;{ }^{1} \mathrm{H}$ NMR $\left(600 \mathrm{MHz}, \mathrm{CDCl}_{3}\right): \delta=6.94(\mathrm{dt}, J=15.5,6.9$ $\mathrm{Hz}, 1 \mathrm{H}), 5.90(\mathrm{~s}, 1 \mathrm{H}), 5.80(\mathrm{dt}, J=15.5,1.5 \mathrm{~Hz}, 1 \mathrm{H}), 5.68(\mathrm{ddd}, J=17.2,10.3,7.6 \mathrm{~Hz}, 1 \mathrm{H})$, $5.24(\mathrm{~m}, 1 \mathrm{H}), 5.21(\mathrm{~m}, 2 \mathrm{H}), 4.94(\mathrm{ddd}, J=11.2,7.7,3.6 \mathrm{~Hz}, 1 \mathrm{H}), 4.72(\mathrm{~d}, J=6.7 \mathrm{~Hz}, 1 \mathrm{H})$, 4.01 (brs, $3 \mathrm{H}$ ), 3.39 (s, $3 \mathrm{H}), 2.65$ (ddq, $J=11.2,8.6,6.8 \mathrm{~Hz}, 1 \mathrm{H}), 2.46$ (dd, $J=14.2,2.9 \mathrm{~Hz}, 1$ H), $2.40(\mathrm{dd}, J=14.2,9.1 \mathrm{~Hz}, 1 \mathrm{H}), 2.25(\mathrm{~m}, 2 \mathrm{H}), 1.84(\mathrm{~d}, J=0.9 \mathrm{~Hz}, 3 \mathrm{H}), 1.62(\mathrm{~s}, 3 \mathrm{H})$, 1.70-1.50 (m, $5 \mathrm{H}), 0.97(\mathrm{~d}, J=6.8 \mathrm{~Hz}, 3 \mathrm{H}), 0.92(\mathrm{~s}, 9 \mathrm{H}), 0.07 \mathrm{ppm}(\mathrm{s}, 6 \mathrm{H}) ;{ }^{13} \mathrm{C}$ NMR $(150$ $\left.\mathrm{MHz}, \mathrm{CDCl}_{3}\right): \delta=166.1,149.1,144.3,138.2,135.8,125.1,121.4,117.4,94.7,77.2,77.0,74.8$ $68.1,55.5,42.1,36.1,34.8,32.1,25.9,24.0,23.9,18.4,16.8,13.9,-5.2$ ppm; HRMS (ESI): calcd for $\mathrm{C}_{28} \mathrm{H}_{49} \mathrm{IO}_{5} \mathrm{SiNa}^{+}\left[\mathrm{M}+\mathrm{Na}^{+}\right]$643.2286, found 643.2274.

Hexaene 80: To a mixture of stannane ent-3 (936 mg, $1.80 \mathrm{mmol})$ and vinyl iodide 81 (864 mg, $1.39 \mathrm{mmol})$ in NMP (15 $\mathrm{mL}$, degassed by using the freeze-thaw process sequence three times) 


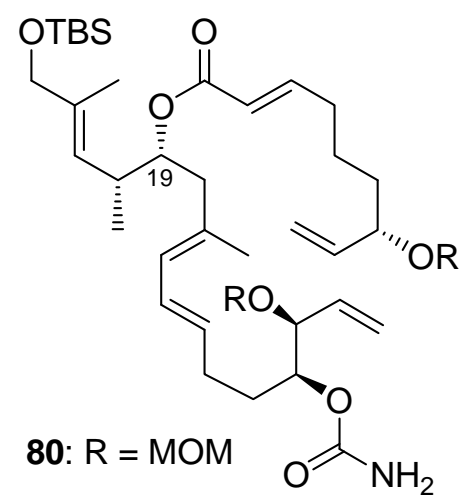

$(2 \times 20 \mathrm{~mL})$, brine $(20 \mathrm{~mL})$, dried $\left(\mathrm{Na}_{2} \mathrm{SO}_{4}\right)$ and concentrated in vacuo. Flash column chromatography (silica gel, hexanes:EtOAc:Et ${ }_{3} \mathrm{~N}$ 3:2:0.01) afforded hexaene 80 (663 mg, 66\%) as a colorless oil. 80: $R_{\mathrm{f}}=0.35$ (silica gel, hexanes:EtOAc $\left.3: 2\right) ;[\alpha]_{\mathrm{D}}{ }^{25}=-2.8\left(\mathrm{CH}_{2} \mathrm{Cl}_{2}, c=0.18\right.$ ); IR (film) $v_{\max } 3356,2930,2857,1719,1443,1385,1255,1098 \mathrm{~cm}^{-1} ;{ }^{1} \mathrm{H}$ NMR (600 MHz, $\left.\mathrm{CDCl}_{3}\right): \delta=6.90(\mathrm{dt}, J=15.6,6.9 \mathrm{~Hz}, 1 \mathrm{H}), 6.19(\mathrm{dd}, J=15.0,10.8 \mathrm{~Hz}, 1 \mathrm{H}), 5.78(\mathrm{~d}, J=15.6$ Hz, $1 \mathrm{H}), 5.74(\mathrm{~d}, J=10.2 \mathrm{~Hz}, 1 \mathrm{H}), 5.72(\mathrm{ddd}, J=17.4,10.2,7.2 \mathrm{~Hz}, 1 \mathrm{H}), 5.68$ (ddd, $J=17.4$, 10.2, $3.0 \mathrm{~Hz}, 1 \mathrm{H}), 5.50(\mathrm{dt}, J=15.0,7.2 \mathrm{~Hz}, 1 \mathrm{H}), 5.32(\mathrm{brd}, J=10.2 \mathrm{~Hz}, 1 \mathrm{H}), 5.31(\mathrm{brd}, J=$ $17.4 \mathrm{~Hz}, 1 \mathrm{H}), 5.25(\mathrm{~d}, J=10.2 \mathrm{~Hz}, 1 \mathrm{H}), 5.21(\mathrm{brd}, J=9.6 \mathrm{~Hz}, 1 \mathrm{H}), 5.20(\mathrm{brd}, J=17.4 \mathrm{~Hz}, 1$ H), 4.95 (ddd, $J=9.0,7.2,4.2 \mathrm{~Hz}, 1 \mathrm{H}), 4.88$ (dt, $J=9.0,4.6 \mathrm{~Hz}, 1 \mathrm{H}), 4.77$ (brs, $2 \mathrm{H}), 4.71(\mathrm{~d}, J$ $=6.6 \mathrm{~Hz}, 1 \mathrm{H}), 4.70(\mathrm{~d}, J=7.2 \mathrm{~Hz}, 1 \mathrm{H}), 4.58(\mathrm{~d}, J=6.6 \mathrm{~Hz}, 1 \mathrm{H}), 4.53(\mathrm{~d}, J=7.2 \mathrm{~Hz}, 1 \mathrm{H}), 4.11$ $(\mathrm{dd}, J=6.6,4.8 \mathrm{~Hz}, 1 \mathrm{H}), 4.00(\mathrm{~s}, 2 \mathrm{H}), 3.99(\mathrm{q}, J=7.2 \mathrm{~Hz}, 1 \mathrm{H}), 3.39$ (s, $3 \mathrm{H}), 3.37$ (s, $3 \mathrm{H})$, $2.64(\mathrm{ddq}, J=9.6,7.2,6.6 \mathrm{~Hz}, 1 \mathrm{H}), 2.30(\mathrm{dd}, J=13.8,3.6 \mathrm{~Hz}, 1 \mathrm{H}), 2.23-2.13(\mathrm{~m}, 5 \mathrm{H})$, 1.77-1.48 (m, $6 \mathrm{H}), 1.72(\mathrm{~s}, 3 \mathrm{H}), 1.58(\mathrm{~s}, 3 \mathrm{H}), 0.96(\mathrm{~d}, J=6.6 \mathrm{~Hz}, 3 \mathrm{H}), 0.93(\mathrm{~s}, 9 \mathrm{H}), 0.06 \mathrm{ppm}$ $(\mathrm{s}, 6 \mathrm{H}) ;{ }^{13} \mathrm{C} \mathrm{NMR}\left(150 \mathrm{MHz}, \mathrm{CDCl}_{3}\right): \delta=166.3,156.5,148.6,138.1,135.3,134.0,132.7,131.3$, $127.5,127.2,125.6,121.6,119.4,117.4,94.0,93.7,77.8,77.0,75.7,75.4,68.2,55.7,55.4,42.5$, 36.0, 34.8, 32.0, 30.4, 28.7, 25.9, 23.8, 18.4, 16.7, 16.6, 13.8, -5.2, -5.2 ppm; HRMS (ESI): calcd for $\mathrm{C}_{39} \mathrm{H}_{67} \mathrm{NO}_{9} \mathrm{SiNa}^{+}\left[\mathrm{M}+\mathrm{Na}^{+}\right]$744.4477, found 744.4463. 
Alcohol 82: To a solution of TBS ether $80(520 \mathrm{mg}, 0.72 \mathrm{mmol})$ in THF $(10 \mathrm{~mL})$ was added TBAF (1.0 M in THF, $1.08 \mathrm{~mL}, 1.08 \mathrm{mmol})$ at room temperature. The reaction mixture was

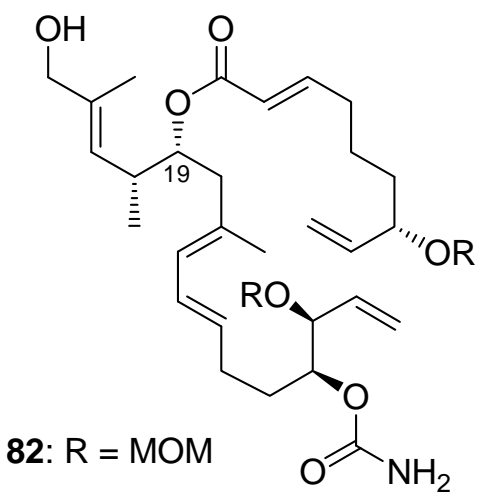
stirred for $2 \mathrm{~h}$ before it was concentrated in vacuo. The resulting residue was diluted with $\mathrm{H}_{2} \mathrm{O}(5 \mathrm{~mL})$ and extracted with EtOAc (3 $\times 20 \mathrm{~mL}$ ). The combined organic layers were washed with brine $(5 \mathrm{~mL})$, dried $\left(\mathrm{Na}_{2} \mathrm{SO}_{4}\right)$ and concentrated in vacuo. Flash column chromatorgraphy (silica gel, hexanes:EtOAc:Et ${ }_{3} \mathrm{~N}$ 7:3:1) afforded alcohol $82(340 \mathrm{mg}, 76 \%)$ as a viscous oil. 82: $R_{\mathrm{f}}=0.35$ (silica gel, hexanes:EtOAc 3:2); $[\alpha]_{\mathrm{D}}{ }^{25}=-19.6\left(\mathrm{CHCl}_{3}, c=1.92\right)$; IR (film) $v_{\max } 3455,2932,1714$, 1386, 1319, 1268, $1029 \mathrm{~cm}^{-1} ;{ }^{1} \mathrm{H}$ NMR $\left(600 \mathrm{MHz}, \mathrm{CDCl}_{3}\right): \delta=6.92(\mathrm{dt}, J=13.8,6.9 \mathrm{~Hz}, 1 \mathrm{H})$, $6.20(\mathrm{dd}, J=15.0,10.8 \mathrm{~Hz}, 1 \mathrm{H}), 5.80(\mathrm{dt}, J=15.7,1.4 \mathrm{~Hz}, 1 \mathrm{H}), 5.75(\mathrm{~d}, J=12.0 \mathrm{~Hz}, 1 \mathrm{H})$, 5.72 (ddd, $J=17.7,10.3,7.3 \mathrm{~Hz}, 1 \mathrm{H}), 5.67$ (ddd, $J=18.0,10.5,7.7 \mathrm{~Hz}, 1 \mathrm{H}), 5.53$ (dt $J=14.3$, $6.9 \mathrm{~Hz}, 1 \mathrm{H}), 5.33(\mathrm{dd}, J=8.6,1.0 \mathrm{~Hz}, 1 \mathrm{H}), 5.32(\mathrm{~d}, J=1.0 \mathrm{~Hz}, 1 \mathrm{H}), 5.26(\mathrm{dd}, J=9.9,1.2 \mathrm{~Hz}$, $1 \mathrm{H}), 5.23$ (bm, $1 \mathrm{H}), 5.20$ (brs, $1 \mathrm{H}), 4.98$ (ddd, $J=11.1,6.9,4.4 \mathrm{~Hz}, 1 \mathrm{H}), 4.88$ (dt, $J=9.0,4.8$ $\mathrm{Hz}, 1 \mathrm{H}), 4.71(\mathrm{~d}, J=4.0 \mathrm{~Hz}, 1 \mathrm{H}), 4.70(\mathrm{~d}, J=4.0 \mathrm{~Hz}, 1 \mathrm{H}), 4.73-4.65$ (brs, $2 \mathrm{H}), 4.59$ (d, $J=$ $6.8 \mathrm{~Hz}, 1 \mathrm{H}), 4.54(\mathrm{~d}, J=6.8 \mathrm{~Hz}, 1 \mathrm{H}), 4.12(\mathrm{bm}, 1 \mathrm{H}), 4.00$ (brs, $3 \mathrm{H}), 3.40$ (s, $3 \mathrm{H}), 3.38$ (s, 3 H), 2.67 (ddq, $J=12.0,11.1,6.8 \mathrm{~Hz}, 1 \mathrm{H}), 2.32-2.13(\mathrm{~m}, 6 \mathrm{H}), 1.74(\mathrm{~s}, 3 \mathrm{H}), 1.66(\mathrm{~d}, J=1.1 \mathrm{~Hz}$, $3 \mathrm{H}), 1.75-1.50(\mathrm{~m}, 7 \mathrm{H}), 0.98 \mathrm{ppm}(\mathrm{d}, J=6.8 \mathrm{~Hz}, 3 \mathrm{H}) ;{ }^{13} \mathrm{C} \mathrm{NMR}\left(150 \mathrm{MHz}, \mathrm{CDCl}_{3}\right): \delta=166.3$, $156.6,148.8,138.1,135.6,134.0,132.5,131.5,127.6,127.3,127.2,121.6,119.4,117.4,94.0$, $93.7,77.8,77.0,75.5,75.3,68.6,55.7,55.4,42.6,36.0,34.8,32.0,30.4,28.7,23.8,16.7,16.4$, 14.0 ppm; HRMS (ESI): calcd for $\mathrm{C}_{33} \mathrm{H}_{53} \mathrm{NO}_{9} \mathrm{Na}^{+}\left[\mathrm{M}+\mathrm{Na}^{+}\right]$630.3613, found 630.3630 . 
Aldehyde 83: To a solution of alcohol $82(310 \mathrm{mg}, 0.498 \mathrm{mmol})$ in $\mathrm{CH}_{2} \mathrm{Cl}_{2}(5.0 \mathrm{~mL})$ was added $\mathrm{NaHCO}_{3}(209 \mathrm{mg}, 2.49 \mathrm{mmol})$ followed by DMP $(253 \mathrm{mg}, 0.598 \mathrm{mmol})$ at room temperature.

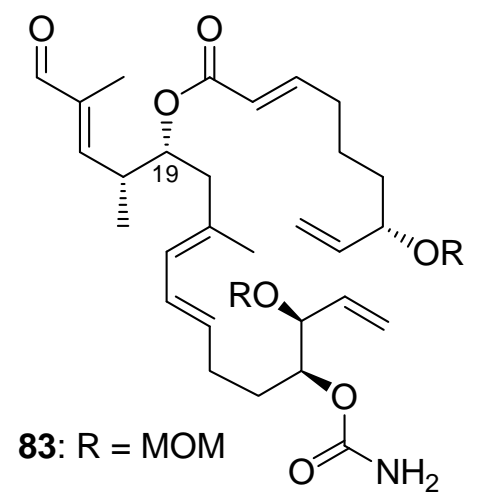
The reaction mixture was stirred for $30 \mathrm{~min}$ before it was quenched with $\mathrm{NaHCO}_{3}\left(5 \mathrm{~mL}\right.$, sat. aq.) and $\mathrm{Na}_{2} \mathrm{~S}_{2} \mathrm{O}_{3}(5 \mathrm{~mL}$, sat. aq.). The resulting mixture was extracted with EtOAc $(3 \times 20 \mathrm{~mL})$, and the combined organic layers were washed with brine $(10 \mathrm{~mL})$, dried $\left(\mathrm{Na}_{2} \mathrm{SO}_{4}\right)$ and concentrated in vacuo. Flash column chromatography (silica gel, hexanes:EtOAc:Et ${ }_{3} \mathrm{~N}$ 1:1:0.01)

afforded aldehyde $83(260 \mathrm{mg}, 84 \%)$ as a colorless viscous oil. 83: $R_{\mathrm{f}}=0.40$ (silica gel, hexanes:EtOAc 3:2); $[\alpha]_{\mathrm{D}}{ }^{25}=-4.2\left(\mathrm{CHCl}_{3}, c=1.0\right)$; IR (film) $v_{\max } 3364,2933,1719,1688,1383$, 1318, $1031 \mathrm{~cm}^{-1} ;{ }^{1} \mathrm{H}$ NMR $\left(600 \mathrm{MHz}, \mathrm{CDCl}_{3}\right): \delta=9.42(\mathrm{~s}, 1 \mathrm{H}), 6.95(\mathrm{dt}, J=13.8,6.9 \mathrm{~Hz}, 1 \mathrm{H})$, $6.33(\mathrm{dd}, J=10.0,1.3 \mathrm{~Hz}, 1 \mathrm{H}), 6.20(\mathrm{dd}, J=15.0,10.8 \mathrm{~Hz}, 1 \mathrm{H}), 5.78(\mathrm{dt}, J=14.1,1.6 \mathrm{~Hz}, 1 \mathrm{H})$, $5.76(\mathrm{~d}, J=10.7 \mathrm{~Hz}, 1 \mathrm{H}), 5.73(\mathrm{ddd}, J=17.5,10.5,7.4 \mathrm{~Hz}, 1 \mathrm{H}), 5.67$ (ddd, $J=17.0,10.5,7.6$ Hz, $1 \mathrm{H}), 5.55$ (dt, $J=14.5,6.9 \mathrm{~Hz}, 1 \mathrm{H}), 5.34$ (d, $J=8.4 \mathrm{~Hz}, 1 \mathrm{H}), 5.31$ (brs, $1 \mathrm{H}), 5.23$ (d, $J=$ $7.3 \mathrm{~Hz}, 1 \mathrm{H}), 5.21(\mathrm{~s}, 1 \mathrm{H}), 5.11$ (ddd, $J=11.7,7.4,5.8 \mathrm{~Hz}, 1 \mathrm{H}) 4.89$ (dt, $J=9.1,4.8 \mathrm{~Hz}, 1 \mathrm{H})$, $4.71(\mathrm{~d}, J=3.7 \mathrm{~Hz}, 1 \mathrm{H}), 4.71(\mathrm{~d}, J=3.7 \mathrm{~Hz}, 1 \mathrm{H}), 4.78($ brs, $2 \mathrm{H}), 4.59(\mathrm{~d}, J=6.8 \mathrm{~Hz}, 1 \mathrm{H})$, $4.12(\mathrm{dd}, J=7.2,4.9 \mathrm{~Hz}, 1 \mathrm{H}), 4.01(\mathrm{bm}, 1 \mathrm{H}), 3.40$ (s, $3 \mathrm{H}), 3.39$ (s, $3 \mathrm{H}), 2.98$ (ddq, $J=11.7$, 10.7, $6.6 \mathrm{~Hz}, 1 \mathrm{H}), 2.30-2.22(\mathrm{~m}, 4 \mathrm{H}), 2.20-2.14(\mathrm{~m}, 2 \mathrm{H}), 1.57(\mathrm{~s}, 3 \mathrm{H}), 1.58(\mathrm{~s}, 3 \mathrm{H})$, 1.78-1.68 (m, $3 \mathrm{H}), 1.70-1.50(\mathrm{~m}, 4 \mathrm{H}), 1.11 \mathrm{ppm}(\mathrm{d}, J=6.6 \mathrm{~Hz}, 3 \mathrm{H}) ;{ }^{13} \mathrm{C}$ NMR $(150 \mathrm{MHz}$, $\left.\mathrm{CDCl}_{3}\right): \delta=195.2,166.0,156.5,154.5,149.5,139.4,138.1,134.0,132.1,131.5,128.2,126.9$ 121.2, 119.4, 117.4, 94.0, 93.7, 77.8, 76.9, 75.3, 74.2, 55.7, 55.5, 42.4, 37.1, 34.8, 32.1, 30.4, 28.7, 23.8, 16.7, 15.3, 9.5 ppm; HRMS (ESI): calcd for $\mathrm{C}_{33} \mathrm{H}_{51} \mathrm{NO}_{9} \mathrm{Na}^{+}\left[\mathrm{M}+\mathrm{Na}^{+}\right] 628.3456$, found 628.3475 . 
Vinyl iodide 84: To a solution of $\mathrm{CrCl}_{2}$ (426 mg, $3.46 \mathrm{mmol}$, flame-dried) in THF (3 mL) was added a solution of the aldehyde $83(215 \mathrm{mg}, 0.35 \mathrm{mmol})$ and $\mathrm{CHI}_{3}(410 \mathrm{mg}, 1.04 \mathrm{mmol})$ in

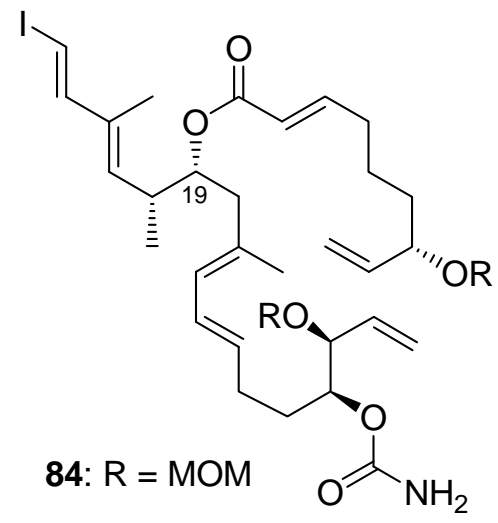
dioxane $(18 \mathrm{~mL})$ at room temperature. The reaction mixture was stirred for $2 \mathrm{~h}$ before it was quenched with $\mathrm{NaHCO}_{3}(5 \mathrm{~mL}$ sat. aq.). The resulting mixture was diluted with EtOAc $(50 \mathrm{~mL})$, filtered through a short pad of Celite ${ }^{\circledR}$ and extracted with EtOAc $(3 \times 20 \mathrm{~mL})$. The combined organic layers were washed with brine $(10 \mathrm{~mL})$, dried $\left(\mathrm{Na}_{2} \mathrm{SO}_{4}\right)$ and concentrated in vacuo. Flash column chromatography (silica gel, hexanes:EtOAc: $\mathrm{Et}_{3} \mathrm{~N}$ 6.5:3.5:0.05) afforded vinyl iodide $\mathbf{8 4}(218 \mathrm{mg}, 79 \%)$ as a colorless gummy solid. $\mathbf{8 4}: R_{\mathrm{f}}=0.45$ (silica gel, hexanes:EtOAc 2:3); $[\alpha]_{\mathrm{D}}^{25}=-41.5\left(\mathrm{CHCl}_{3}, c=1.0\right)$; IR (film) $v_{\max } 3456,2936,1718$, 1386, 1318, 1097, $1032 \mathrm{~cm}^{-1} ;{ }^{1} \mathrm{H}$ NMR (600 MHz, $\left.\mathrm{CDCl}_{3}\right): \delta=7.05$ (d, $\left.J=14.6 \mathrm{~Hz}, 1 \mathrm{H}\right), 6.92$ $(\mathrm{dt}, J=13.9,6.9 \mathrm{~Hz}, 1 \mathrm{H}), 6.21(\mathrm{~d}, J=14.6 \mathrm{~Hz}, 1 \mathrm{H}), 6.20(\mathrm{dd}, J=13.9,10.7 \mathrm{~Hz}, 1 \mathrm{H}), 5.79(\mathrm{dt}$, $J=15.6 \mathrm{~Hz}, 1 \mathrm{H}), 5.74(\mathrm{~d}, J=11.8 \mathrm{~Hz}, 1 \mathrm{H}), 5.73$ (ddd, $J=17.7,10.4,7.4 \mathrm{~Hz}, 1 \mathrm{H}), 5.68$ (ddd, $J$ $=18.1,10.5,7.7 \mathrm{~Hz}, 1 \mathrm{H}), 5.53(\mathrm{dt}, J=14.1,6.8 \mathrm{~Hz}, 1 \mathrm{H}), 5.35-5.30(\mathrm{bm}, 2 \mathrm{H}), 5.25-5.19(\mathrm{bm}$, $2 \mathrm{H}), 4.97$ (ddd, $J=10.9,6.8,4.2 \mathrm{~Hz}, 1 \mathrm{H}), 4.89$ (dt, $J=9.1,4.8 \mathrm{~Hz}, 1 \mathrm{H}), 4.72(\mathrm{~d}, J=4.2 \mathrm{~Hz}, 1$ H), $4.71(\mathrm{~d}, J=4.2 \mathrm{~Hz}, 1 \mathrm{H}), 4.75-4.52(\mathrm{brs}, 2 \mathrm{H}), 4.59(\mathrm{~d}, J=3.6 \mathrm{~Hz}, 1 \mathrm{H}), 4.55(\mathrm{~d}, J=6.6 \mathrm{~Hz}$, $1 \mathrm{H}), 4.12(\mathrm{dd}, J=7.3,5.0 \mathrm{~Hz}, 1 \mathrm{H}), 4.00(\mathrm{bm}, 1 \mathrm{H}), 3.41(\mathrm{~s}, 3 \mathrm{H}), 3.36(\mathrm{~s}, 3 \mathrm{H}), 2.74$ (ddq, $J=$ 11.8, 10.9, $6.6 \mathrm{~Hz}, 1 \mathrm{H}), 2.31-2.10(\mathrm{~m}, 6 \mathrm{H}), 1.73(\mathrm{~s}, 3 \mathrm{H}), 1.71(\mathrm{~d}, J=1.2 \mathrm{~Hz}, 3 \mathrm{H}), 1.77-1.50$ $(\mathrm{m}, 7 \mathrm{H}), 1.00 \mathrm{ppm}(\mathrm{d}, J=6.6 \mathrm{~Hz}, 3 \mathrm{H}) ;{ }^{13} \mathrm{C} \mathrm{NMR}\left(150 \mathrm{MHz}, \mathrm{CDCl}_{3}\right): \delta=166.2,156.5,149.5$, 149.0, 138.1, 135.4, 134.8, 134.0, 132.2, 131.6. 127.8, 127.1, 121.5, 119.4, 117.4, 94.0, 93.7, $77.8,77.0,75.4,75.1,74.2,55.7,55.5,42.4,36.4,34.8,32.0,30.4,28.7,23.8,16.7,16.3,12.3$ ppm; HRMS (ESI): calcd for $\mathrm{C}_{34} \mathrm{H}_{52} \mathrm{INO}_{8} \mathrm{Na}^{+}\left[\mathrm{M}+\mathrm{Na}^{+}\right]$752.2630, found 752.2654. 
Diol 85 and cyclic carbonate: To a solution of bis-MOM ether $84(150 \mathrm{mg}, 0.201 \mathrm{mmol})$ in $\mathrm{MeOH}(15 \mathrm{~mL})$ was added TMSCl $(207 \mu \mathrm{L}, 2.01 \mathrm{mmol})$ at $40{ }^{\circ} \mathrm{C}$. The reaction mixture was

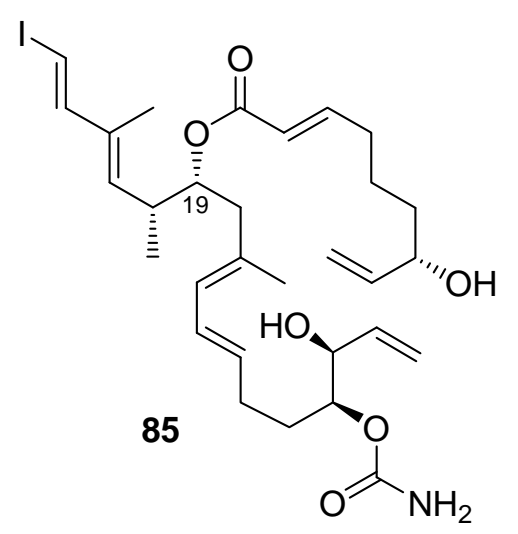
stirred for $1 \mathrm{~h}$ before it was cooled to room temperature, quenched with $\mathrm{NaHCO}_{3}(300 \mathrm{mg})$ and concentrated in vacuo. The resulting residue was dissolved in EtOAc $(15 \mathrm{~mL})$, filtered through a pad of Celite ${ }^{\circledR}$, washed with EtOAc $(2 \times 10 \mathrm{~mL})$ and concentrated in vacuo. Flash column chromatography (silica gel, hexanes:EtOAc: $\mathrm{Et}_{3} \mathrm{~N}$ 4:6:0.025 $\rightarrow$ 0:10:0.05) afforded diol 85 (86 $\mathrm{mg}, 67 \%)$ as a colorless gummy solid and cyclic carbonate $\mathbf{8 6}(12.8 \mathrm{mg}, 10 \%)$ as a gummy solid. 85: $R_{\mathrm{f}}=0.36$ (silica gel, hexanes:EtOAc 3:2); $[\alpha]_{\mathrm{D}}{ }^{25}=+30.8\left(\mathrm{CHCl}_{3, c}=1.0\right)$; IR (film) $v_{\max }$ 3365, 2931, 1709, 1651, 1390, 1318, 1181, 1059, $991 \mathrm{~cm}^{-1} ;{ }^{1} \mathrm{H}$ NMR $\left(600 \mathrm{MHz}, \mathrm{CDCl}_{3}\right): \delta=$ $7.06(\mathrm{~d}, J=14.6 \mathrm{~Hz}, 1 \mathrm{H}), 6.92(\mathrm{ddd}, J=15.6,6.9,6.9 \mathrm{~Hz}, 1 \mathrm{H}), 6.22(\mathrm{~d}, J=14.6 \mathrm{~Hz}, 1 \mathrm{H}), 6.19$ (dd, $J=15.0,10.8 \mathrm{~Hz}, 1 \mathrm{H}), 5.91-5.84(\mathrm{~m}, 2 \mathrm{H}), 5.79(\mathrm{~d}, J=15.6 \mathrm{~Hz}, 1 \mathrm{H}), 5.73(\mathrm{~d}, J=10.8 \mathrm{~Hz}$ $1 \mathrm{H}), 5.51(\mathrm{ddd}, J=14.5,6.9 .6 .9 \mathrm{~Hz}, 1 \mathrm{H}), 5.38(\mathrm{~d}, J=17.1 \mathrm{~Hz}, 1 \mathrm{H}), 5.32(\mathrm{~d}, J=12.9 \mathrm{~Hz}, 1 \mathrm{H})$, 5.27-5.22 (m, $2 \mathrm{H}), 5.13(\mathrm{~d}, J=10.4 \mathrm{~Hz}, 1 \mathrm{H}), 4.97$ (ddd, $J=10.5,7.0,3.7 \mathrm{~Hz}, 1 \mathrm{H}), 4.80-4.65$ (bm, $2 \mathrm{H}), 4.75$ (ddd, $J=14.1,9.4,4.8 \mathrm{~Hz}, 1 \mathrm{H}), 4.20-4.10$ (m, $2 \mathrm{H}), 2.78-2.70(\mathrm{~m}, 1 \mathrm{H})$, 2.30-2.22 (m, 2 H), 2.20-2.10 (m, 4 H), 1.77-1.66 (m, 2 H), 1.64-1.47 (m, 4 H), 1.73 (s, 3 H), $1.72(\mathrm{~s}, 3 \mathrm{H}), 1.00(\mathrm{~d}, J=6.8 \mathrm{~Hz}, 3 \mathrm{H}), 1.04-0.96(\mathrm{~m}, 1 \mathrm{H}), 0.90-0.80 \mathrm{ppm}(\mathrm{m}, 1 \mathrm{H}) ;{ }^{13} \mathrm{C} \mathrm{NMR}$ $\left(150 \mathrm{MHz}, \mathrm{CDCl}_{3}\right): \delta=166.2,157.0,149.5,149.0,141.0,136.9,135.3,134.9,132.3,131.5$ $127.8,127.2,121.4,117.0,114.8,76.9,75.0,74.2,74.2,72.8,42.6,36.6,36.3,32.0,30.4,28.7$, 23.9, 16.7, 16.4, 12.3 ppm; HRMS (ESI): calcd for $\mathrm{C}_{30} \mathrm{H}_{44} \mathrm{INO}_{6} \mathrm{Na}^{+}\left[\mathrm{M}+\mathrm{Na}^{+}\right]$664.2106, found 664.2118. 


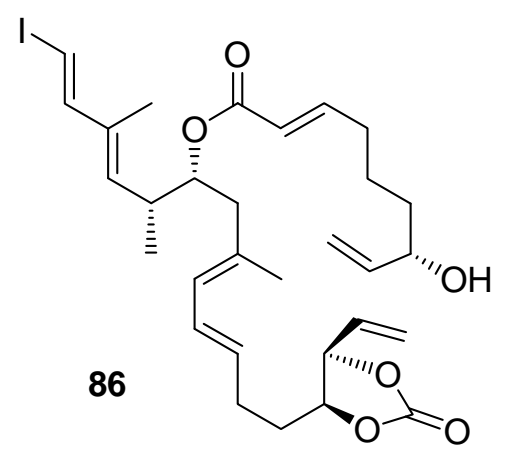

Cyclic carbonate 86: $R_{\mathrm{f}}=0.33$ (silica gel, hexanes:EtOAc 7:3); $[\alpha]_{\mathrm{D}}^{25}=-41.6\left(\mathrm{CHCl}_{3}, c=1.0\right) ;$ IR (film) $v_{\max } 3493,2932,1803$, 1711, 1432, 1269, 1187, 1048, $988 \mathrm{~cm}^{-1}$; ${ }^{1} \mathrm{H}$ NMR $(600 \mathrm{MHz}$, $\left.\mathrm{CDCl}_{3}\right): \delta=7.05(\mathrm{~d}, J=14.0 \mathrm{~Hz}, 1 \mathrm{H}), 6.92(\mathrm{dt}, J=15.6,7.2 \mathrm{~Hz}$, $1 \mathrm{H}), 6.22(\mathrm{~d}, J=9.0 \mathrm{~Hz}, 1 \mathrm{H}), 6.23(\mathrm{~m}, 1 \mathrm{H}), 5.79(\mathrm{td}, J=15.7$,

$1.5 \mathrm{~Hz}, 1 \mathrm{H}), 5.74(\mathrm{~d}, J=10.7 \mathrm{~Hz}, 1 \mathrm{H}), 5.50(\mathrm{~m}, 2 \mathrm{H}), 5.44(\mathrm{~m}, 2 \mathrm{H}), 5.31(\mathrm{~d}, J=9.9 \mathrm{~Hz}, 1 \mathrm{H})$, $5.24(\operatorname{td}, J=16.8,1.2 \mathrm{~Hz}, 1 \mathrm{H}), 5.13(\operatorname{td}, J=10.2,1.2 \mathrm{~Hz}, 1 \mathrm{H}), 4.97(\mathrm{ddd}, J=10.8,7.0,3.9 \mathrm{~Hz}$, $1 \mathrm{H}), 4.66(\mathrm{t}, J=7.2 \mathrm{~Hz}, 1 \mathrm{H}), 4.32(\mathrm{dt}, J=8.0,4.6 \mathrm{~Hz}, 1 \mathrm{H}), 4.12(\mathrm{bm}, 1 \mathrm{H}), 2.73(\mathrm{~m}, 1 \mathrm{H})$, 2.35-2.17 (m, $4 \mathrm{H}), 1.91-1.83$ (m, $1 \mathrm{H}), 1.82-1.72(\mathrm{~m}, 2 \mathrm{H}), 1.74$ (s, $3 \mathrm{H}), 1.70$ (d, $J=1.1 \mathrm{~Hz}, 3$ H), 1.69 (brs, $2 \mathrm{H}), 1.61-1.49$ (m, $4 \mathrm{H}), 1.02(\mathrm{~m}, 1 \mathrm{H}), 0.99 \mathrm{ppm}(\mathrm{d}, J=6.7 \mathrm{~Hz}, 3 \mathrm{H}) ;{ }^{13} \mathrm{C} \mathrm{NMR}$ $\left(150 \mathrm{MHz}, \mathrm{CDCl}_{3}\right): \delta=166.1,154.2,149.5,149.0,141.0,135.3,134.9,133.4,132.0,129.4$ $128.4,127.2,121.4,121.4,114.9,82.6,81.1,75.0,74.3,72.9,42.5,36.6,36.3,32.8,32.0,28.0$, 23.8, 16.8, 16.4, 12.3 ppm; HRMS (ESI): calcd for $\mathrm{C}_{30} \mathrm{H}_{41} \mathrm{IO}_{6} \mathrm{Na}^{+}\left[\mathrm{M}+\mathrm{Na}^{+}\right]$647.1840, found 647.1823.

Macrocycle 87: To a solution of diol $85(80 \mathrm{mg}, 0.12 \mathrm{mmol})$ in $\mathrm{CH}_{2} \mathrm{Cl}_{2}(25 \mathrm{~mL}, 0.005 \mathrm{M})$ was added Grubbs II catalyst $(5.2 \mathrm{mg}, 6.1 \mu \mathrm{mol})$ at room temperature. The reaction mixture was

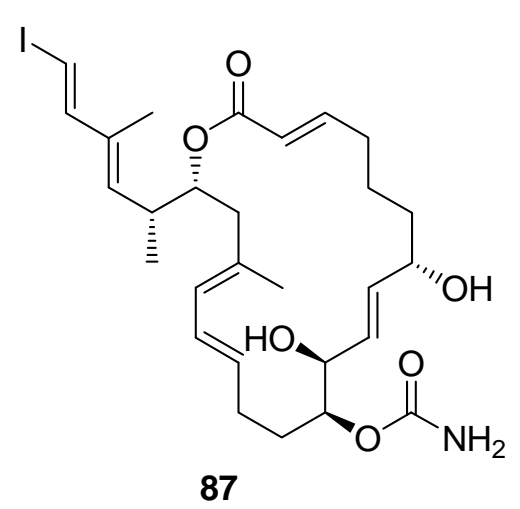
stirred for $1 \mathrm{~h}$ before it was concentrated in vacuo. Flash column chromatography (silica gel, hexanes:EtOAc:Et ${ }_{3} \mathrm{~N}$ 3:7:0.05 $\rightarrow$ EtOAc:MeOH:Et ${ }_{3} \mathrm{~N}$ 9.5:0.5:0.05) afforded the macrocycle 87 (62 mg, 81\%) as white gummy solid. 87: $R_{\mathrm{f}}=0.31$ (silica gel, hexanes:EtOAc 8:1); $[\alpha]_{\mathrm{D}}^{25}=-28.5\left(\mathrm{CHCl}_{3}, c=1.0\right)$ IR (film) $V_{\max } 3355,2924,2854,1714,1392,1316,1219,1076,772 \mathrm{~cm}^{-1}$; 
${ }^{1} \mathrm{H}$ NMR $\left(600 \mathrm{MHz}, \mathrm{CDCl}_{3}\right): \delta=7.07(\mathrm{~d}, J=14.6 \mathrm{~Hz}, 1 \mathrm{H}), 6.84(\mathrm{ddd}, J=15.2,9.7,5.4 \mathrm{~Hz}, 1$ H), $6.24(\mathrm{~d}, J=14.6 \mathrm{~Hz}, 1 \mathrm{H}), 6.14(\mathrm{dd}, J=14.9,11.2 \mathrm{~Hz}, 1 \mathrm{H}), 5.79(\mathrm{dd}, J=15.6,7.3 \mathrm{~Hz}, 1 \mathrm{H})$, $5.76(\mathrm{~d}, J=15.4 \mathrm{~Hz}, 1 \mathrm{H}), 5.68(\mathrm{dd}, J=15.6,13.1 \mathrm{~Hz}, 1 \mathrm{H}), 5.67$ (d, $J=10.6 \mathrm{~Hz}, 1 \mathrm{H}), 5.48$ (ddd, $J=14.8,9.8,5.0 \mathrm{~Hz}, 1 \mathrm{H}), 5.31(\mathrm{~d}, J=9.6 \mathrm{~Hz}, 1 \mathrm{H}), 4.97(\mathrm{ddd}, J=10.0,6.1,1.9 \mathrm{~Hz}, 1 \mathrm{H})$, 4.80-4.72 (brs, $2 \mathrm{H}), 4.70$ (ddd, $J=10.2,6.3,2.4 \mathrm{~Hz}, 1 \mathrm{H}), 4.31$ (bm, $1 \mathrm{H}), 4.13$ (m, $1 \mathrm{H}), 2.72$ (m, $1 \mathrm{H}), 2.27-2.15(\mathrm{~m}, 2 \mathrm{H}), 2.16-2.06(\mathrm{~m}, 4 \mathrm{H}), 1.76(\mathrm{~s}, 3 \mathrm{H}), 1.71(\mathrm{~s}, 3 \mathrm{H}), 1.67-1.58(\mathrm{~m}, 3$ H), 1.54-1.44 (m, $5 \mathrm{H}), 0.99 \mathrm{ppm}(\mathrm{d}, J=6.7 \mathrm{~Hz}, 3 \mathrm{H}) ;{ }^{13} \mathrm{C} \mathrm{NMR}\left(150 \mathrm{MHz}, \mathrm{CDCl}_{3}\right): \delta=166.3$, $156.9,149.5,148.8,135.2,135.0,134.2,132.0,131.2,129.6,128.0,127.2,121.3,74.4,74.0$ 73.0, 72.3, 45.8, 43.7, 37.4, 36.8, 32.7, 30.3, 28.9, 24.7, 16.9, 16.4, 12.4 ppm; HRMS (ESI): calcd for $\mathrm{C}_{28} \mathrm{H}_{40} \mathrm{INO}_{6} \mathrm{Na}^{+}\left[\mathrm{M}+\mathrm{Na}^{+}\right]$636.1793, found 636.1810.

Palmerolide A isomer ent-(19-epi-20-epi-1) and decarbamated palmerolide A isomer 88: To a mixture of vinyl iodide 87 (20 mg, $32.6 \mu \mathrm{mol}), 42$ (6.4 mg, $65.2 \mu \mathrm{mol}), \mathrm{K}_{2} \mathrm{CO}_{3}(28 \mathrm{mg}, 196.0$

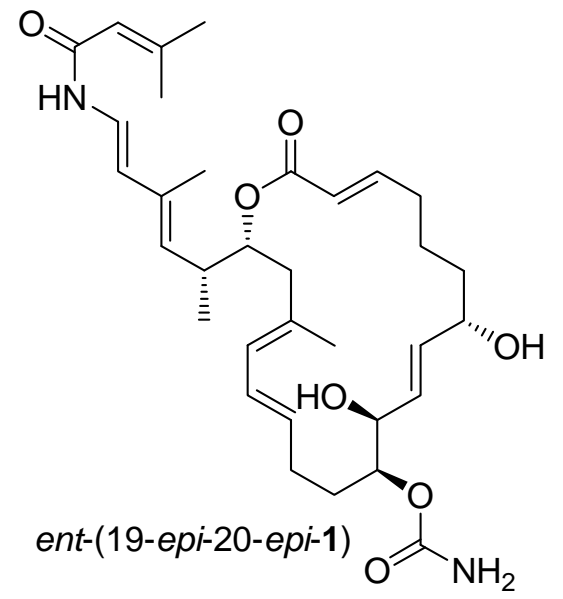
$\mu \mathrm{mol})$ and $\mathrm{CuI}(9.2 \mathrm{mg}, 48.8 \mu \mathrm{mol})$ in $\operatorname{DMF}(2.0 \mathrm{~mL})$ was added $N, N^{\prime}$-dimethylethylenediamine $(10.8 \mu \mathrm{L}, 97.8 \mu \mathrm{mol})$ at room temperature. The reaction mixture was stirred for $1 \mathrm{~h}$ before it was concentrating in vacuo. The resulting residue was diluted with EtOAc $(10 \mathrm{~mL})$, filtered through a pad of Celite $^{\circledR}$, washed with EtOAc $(10 \mathrm{~mL})$ and the filtrate was concentrated in vacuo. Preparative TLC purification (silica gel, EtOAc: $\mathrm{Et}_{3} \mathrm{~N}$ 100:1) afforded ent-(19-epi-20-epi-1) $(8.8 \mathrm{mg}, 46 \%)$ as a gummy solid and decarbamated product $88(0.9 \mathrm{mg}, \sim 5 \%)$ as a gummy solid. Palmerolide A isomer ent-(19-epi20-epi-1): $R_{\mathrm{f}}=0.23$ (silica gel, hexanes:EtOAc 0:1); $[\alpha]_{\mathrm{D}}{ }^{25}=-95.0(\mathrm{MeOH}, c=0.12){ }^{2}$ IR (film) 
$v_{\max } 3390,2919,2850,1712,1640,1456,1377,1259,1145 \mathrm{~cm}^{-1} ;{ }^{1} \mathrm{H}$ NMR (600 MHz, DMSO$\left.d^{6}\right): \delta=9.85(\mathrm{~d}, J=10.4 \mathrm{~Hz}, 1 \mathrm{H}), 6.85(\mathrm{dd}, J=14.5,10.4 \mathrm{~Hz}, 1 \mathrm{H}), 6.71(\mathrm{ddd}, J=15.2,10.1$, $5.0 \mathrm{~Hz}, 1 \mathrm{H}), 6.50$ (bd, $2 \mathrm{H}), 6.05$ (dd, $J=15.1,10.8 \mathrm{~Hz}, 1 \mathrm{H}), 5.84$ (d, $J=14.5 \mathrm{~Hz}, 1 \mathrm{H}), 5.77$ (d, $J=15.7 \mathrm{~Hz}, 1 \mathrm{H}), 5.69$ (brs, $1 \mathrm{H}), 5.60(\mathrm{~d}, J=10.3 \mathrm{~Hz}, 1 \mathrm{H}), 5.55(\mathrm{dd}, J=15.5,8.2 \mathrm{~Hz}, 1 \mathrm{H})$, $5.47(\mathrm{dd}, J=15.5,2.8 \mathrm{~Hz}, 1 \mathrm{H}), 5.41(\mathrm{ddd}, J=14.8,9.6,4.8 \mathrm{~Hz}, 1 \mathrm{H}), 5.18(\mathrm{~d}, J=4.9 \mathrm{~Hz}, 1 \mathrm{H})$, $5.13(\mathrm{~d}, J=9.6 \mathrm{~Hz}, 1 \mathrm{H}), 4.84(\mathrm{ddd}, J=10.0,8.0,1.6 \mathrm{~Hz}, 1 \mathrm{H}), 4.69(\mathrm{~d}, J=2.8 \mathrm{~Hz}, 1 \mathrm{H}), 4.49$ (ddd, $J=10.8,4.8,1.6 \mathrm{~Hz}, 1 \mathrm{H}), 4.14(\mathrm{brs}, 1 \mathrm{H}), 3.82(\mathrm{~m}, 1 \mathrm{H}), 2.69(\mathrm{~m}, 1 \mathrm{H}), 2.16(\mathrm{~m}, 1 \mathrm{H})$, $2.12(\mathrm{~s}, 3 \mathrm{H}), 2.10(\mathrm{~m}, 2 \mathrm{H}), 1.95(\mathrm{~m}, 2 \mathrm{H}), 1.99(\mathrm{~m}, 1 \mathrm{H}), 1.83(\mathrm{~s}, 3 \mathrm{H}), 1.71(\mathrm{~s}, 3 \mathrm{H}), 1.61(\mathrm{~s}, 3$ H), $1.59(\mathrm{~m}, 1 \mathrm{H}), 1.47(\mathrm{~m}, 1 \mathrm{H}), 1.30(\mathrm{~m}, 2 \mathrm{H}), 1.04(\mathrm{~m}, 1 \mathrm{H}), 0.97(\mathrm{~m}, 1 \mathrm{H}), 0.89 \mathrm{ppm}(\mathrm{d}, J=$ $6.8 \mathrm{~Hz}, 3 \mathrm{H}) ;{ }^{13} \mathrm{C}$ NMR $\left(150 \mathrm{MHz}\right.$, DMSO- $\left.d^{6}\right): \delta=165.8,163.6,157.1,152.3,149.7,134.1$, 133.1, 132.4, 132.1, 130.3, 129.4, 128.2, 126.9, 122.6, 121.0, 118.6, 116.9, 75.6, 74.3, 73.0, 69.7, 43.7, 38.2, 37.1, 32.8, 29.9, 29.8, 27.5, 25.4, 20.1, 17.6, 16.6, 13.1 ppm; HRMS (ESI): calcd for $\mathrm{C}_{33} \mathrm{H}_{48} \mathrm{~N}_{2} \mathrm{O}_{7} \mathrm{Na}^{+}\left[\mathrm{M}+\mathrm{Na}^{+}\right]$607.3354, found 607.3367. 


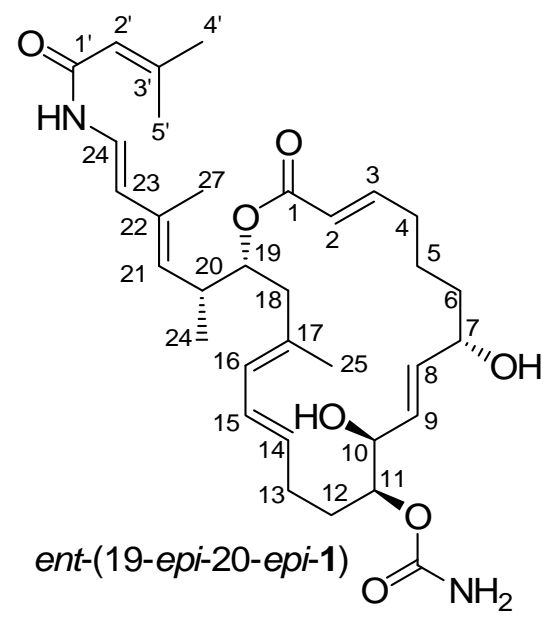

Table 1. NMR Comparision for Natural versus Synthetic Palmerolide A

\begin{tabular}{|c|c|c|c|c|}
\hline Position & $\begin{array}{c}\left.\text { Authentic (DMSO- } d^{6}, 600 \mathrm{MHz}\right)^{3 \mathrm{a}} \\
\delta{ }^{1} \mathrm{H} \\
{[\mathrm{ppm}, \mathrm{mult}, J(\mathrm{~Hz})]}\end{array}$ & $\begin{array}{c}\left.\text { Synthetic (DMSO- } d^{6}, 600 \mathrm{MHz}\right) \\
\delta^{1} \mathrm{H} \\
{[\mathrm{ppm}, \text { mult, } J(\mathrm{~Hz})]}\end{array}$ & 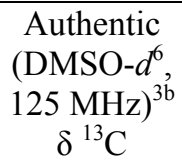 & $\begin{array}{c}\text { Synthetic } \\
\text { (DMSO- } d^{6} \text {, } \\
150 \mathrm{MHz}) \\
\delta{ }^{13} \mathrm{C}\end{array}$ \\
\hline 1 & & & 166.1 & 165.8 \\
\hline 2 & $5.77(\mathrm{~d}, J=15.7 \mathrm{~Hz}, 1 \mathrm{H})$ & $5.77(\mathrm{~d}, J=15.7 \mathrm{~Hz}, 1 \mathrm{H})$ & 121.3 & 121.0 \\
\hline 3 & $6.71(\mathrm{ddd}, J=15.2,10.1,5.0 \mathrm{~Hz}, 1 \mathrm{H})$ & $6.71(\mathrm{ddd}, J=15.2,10.1,5.0 \mathrm{~Hz}, 1 \mathrm{H})$ & 150.0 & 149.7 \\
\hline 4 & $2.10(\mathrm{~m}, 2 \mathrm{H})$ & $2.10(\mathrm{~m}, 2 \mathrm{H})$ & 32.6 & 32.8 \\
\hline \multirow[t]{2}{*}{5} & $1.30(\mathrm{~m}, 1 \mathrm{H})$ & $1.30(\mathrm{~m}, 1 \mathrm{H})$ & 25.7 & 25.4 \\
\hline & $1.04(\mathrm{~m}, 1 \mathrm{H})$ & $1.04(\mathrm{~m}, 1 \mathrm{H})$ & & \\
\hline \multirow[t]{2}{*}{6} & $1.47(\mathrm{~m}, 1 \mathrm{H})$ & $1.47(\mathrm{~m}, 1 \mathrm{H})$ & 38.5 & 38.2 \\
\hline & $1.30(\mathrm{~m}, 1 \mathrm{H})$ & $1.30(\mathrm{~m}, 1 \mathrm{H})$ & & \\
\hline 7 & $3.82(\mathrm{~m}, 1 \mathrm{H})$ & $3.82(\mathrm{~m}, 1 \mathrm{H})$ & 74.5 & 74.3 \\
\hline 8 & $5.55(\mathrm{dd}, J=15.5,8.2 \mathrm{~Hz}, 1 \mathrm{H})$ & $5.55(\mathrm{dd}, J=15.5,8.2 \mathrm{~Hz}, 1 \mathrm{H})$ & 134.3 & 134.1 \\
\hline 9 & $5.47(\mathrm{dd}, J=15.5,2.8 \mathrm{~Hz}, 1 \mathrm{H})$ & $5.47(\mathrm{dd}, J=15.5,2.8 \mathrm{~Hz}, 1 \mathrm{H})$ & 129.6 & 129.4 \\
\hline 10 & 4.14 (brs, $1 \mathrm{H})$ & 4.14 (brs, $1 \mathrm{H})$ & 69.9 & 69.7 \\
\hline 11 & $4.49(\mathrm{ddd}, J=10.8,4.8,1.6 \mathrm{~Hz}, 1 \mathrm{H})$ & $4.49(\mathrm{ddd}, J=10.8,4.8,1.6 \mathrm{~Hz}, 1 \mathrm{H})$ & 73.2 & 73.0 \\
\hline \multirow[t]{2}{*}{12} & $1.59(\mathrm{~m}, 1 \mathrm{H})$ & $1.59(\mathrm{~m}, 1 \mathrm{H})$ & 30.1 & 29.9 \\
\hline & $0.97(\mathrm{~m}, 1 \mathrm{H})$ & $0.97(\mathrm{~m}, 1 \mathrm{H})$ & & \\
\hline 13 & $1.95(\mathrm{~m}, 2 \mathrm{H})$ & $1.95(\mathrm{~m}, 2 \mathrm{H})$ & 30.1 & 29.8 \\
\hline 14 & $5.41(\mathrm{ddd}, 14.8,9.6,4.8 \mathrm{~Hz}, 1 \mathrm{H})$ & $5.41(\mathrm{ddd}, 14.8,9.6,4.8 \mathrm{~Hz}, 1 \mathrm{H})$ & 132.7 & 132.4 \\
\hline 15 & $6.05(\mathrm{dd}, J=15.1,10.8 \mathrm{~Hz}, 1 \mathrm{H})$ & $6.05(\mathrm{dd}, J=15.1,10.8 \mathrm{~Hz}, 1 \mathrm{H})$ & 128.4 & 128.2 \\
\hline 16 & $5.60(\mathrm{~d}, J=10.3 \mathrm{~Hz}, 1 \mathrm{H})$ & $5.60(\mathrm{~d}, J=10.3 \mathrm{~Hz}, 1 \mathrm{H})$ & 127.1 & 126.9 \\
\hline
\end{tabular}




\begin{tabular}{|c|c|c|c|c|}
\hline 17 & & & 132.3 & 132.1 \\
18 & $2.16(\mathrm{~m}, 1 \mathrm{H})$ & 43.9 & 43.7 \\
19 & $1.99(\mathrm{~m}, 1 \mathrm{H})$ & $2.16(\mathrm{~m}, 1 \mathrm{H})$ & \\
20 & $4.84(\mathrm{ddd}, J=10.0,8.0,1.6 \mathrm{~Hz}, 1 \mathrm{H})$ & $1.99(\mathrm{~m}, 1 \mathrm{H})$ & 75.8 & 75.6 \\
21 & $2.69(\mathrm{~m}, 1 \mathrm{H})$ & 2.87 .3 & 37.1 \\
22 & $5.13(\mathrm{~d}, J=9.6 \mathrm{~Hz}, 1 \mathrm{H})$ & $5.13(\mathrm{~d}, J=9.6 \mathrm{~Hz}, 1 \mathrm{H})$ & 130.5 & 130.3 \\
23 & & & 133.3 & 133.1 \\
24 & $5.84(\mathrm{~d}, J=14.5 \mathrm{~Hz}, 1 \mathrm{H})$ & $5.84(\mathrm{~d}, J=14.5 \mathrm{~Hz}, 1 \mathrm{H})$ & 117.2 & 116.9 \\
25 & $6.85(\mathrm{dd}, J=14.5,10.4 \mathrm{~Hz}, 1 \mathrm{H})$ & $6.85(\mathrm{dd}, J=14.5,10.4 \mathrm{~Hz}, 1 \mathrm{H})$ & 122.9 & 122.6 \\
26 & $1.61(\mathrm{~s}, 3 \mathrm{H})$ & $1.61(\mathrm{~s}, 3 \mathrm{H})$ & 16.9 & 16.6 \\
27 & $0.89(\mathrm{~d}, J=6.8 \mathrm{~Hz}, 3 \mathrm{H})$ & $0.89(\mathrm{~d}, J=6.8 \mathrm{~Hz}, 3 \mathrm{H})$ & 17.7 & 17.6 \\
1 & $1.71(\mathrm{~s}, 3 \mathrm{H})$ & $1.71(\mathrm{~s}, 3 \mathrm{H})$ & 13.3 & 13.1 \\
2 & & & 163.9 & 163.6 \\
3 & & $5.69(\mathrm{br} \mathrm{s}, 1 \mathrm{H})$ & 118.8 & 118.6 \\
4 & & & 152.5 & 152.3 \\
5 & $5.69(\mathrm{br} \mathrm{s}, 1 \mathrm{H})$ & $1.83(\mathrm{~s}, 3 \mathrm{H})$ & 27.7 & 27.5 \\
$\mathrm{CONH}$ & & $2.12(\mathrm{~s}, 3 \mathrm{H})$ & 20.4 & 20.1 \\
$24-\mathrm{NH}$ & $9.85(\mathrm{~d}, J=10.4 \mathrm{~Hz}, 1 \mathrm{H})$ & $6.50(\mathrm{br}, 2 \mathrm{H})$ & 157.3 & 157.1 \\
$10-\mathrm{OH}$ & $5.18(\mathrm{~d}, J=4.9 \mathrm{~Hz}, 1 \mathrm{H})$ & $9.85(\mathrm{~d}, J=10.4 \mathrm{~Hz}, 1 \mathrm{H})$ & & \\
$7-\mathrm{OH}$ & $4.69(\mathrm{~d}, J=2.8 \mathrm{~Hz}, 1 \mathrm{H})$ & $5.18(\mathrm{~d}, J=4.9 \mathrm{~Hz}, 1 \mathrm{H})$ & & \\
& & $4.69(\mathrm{~d}, J=2.8 \mathrm{~Hz}, 1 \mathrm{H})$ & & \\
\hline
\end{tabular}

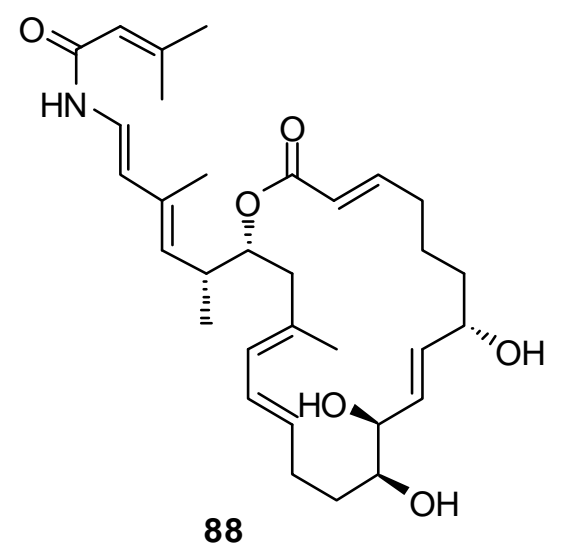

Decarbamated palmerolide A isomer 88: $R_{\mathrm{f}}=0.32$ (silica gel, hexanes:EtOAc 0:1); $[\alpha]_{\mathrm{D}}{ }^{25}=-64.0(\mathrm{MeOH}, c=0.16)$; IR (film) $v_{\max } 3330,2928,1695,1638,1522,1262,1194,1018$ $\mathrm{cm}^{-1} ;{ }^{1} \mathrm{H}$ NMR $\left(600 \mathrm{MHz}, \mathrm{DMSO}-d^{6}\right): \delta=9.85(\mathrm{~d}, J=10.8$ $\mathrm{Hz}, 1 \mathrm{H}), 6.85(\mathrm{dd}, J=15.0,10.2 \mathrm{~Hz}, 1 \mathrm{H}), 6.72(\mathrm{dt}, J=15.0$, 9.6, $4.8 \mathrm{~Hz}, 1 \mathrm{H}), 6.05(\mathrm{dd}, J=14.4,5.4 \mathrm{~Hz}, 1 \mathrm{H}), 5.85(\mathrm{~d}, J=$ $14.4 \mathrm{~Hz}, 1 \mathrm{H}), 5.76(\mathrm{~d}, J=15.6 \mathrm{~Hz}, 1 \mathrm{H}), 5.69(\mathrm{~s}, 1 \mathrm{H}), 5.60$ (d, $J=11.4 \mathrm{~Hz}, 1 \mathrm{H}), 5.57$ (dd, $J=$ 15.6, $3.0 \mathrm{~Hz}, 1 \mathrm{H}), 5.49$ (ddd, $J=15.6,7.8,1.8 \mathrm{~Hz}, 1 \mathrm{H}), 5.42(\mathrm{ddd}, J=13.8,10.2,4.2 \mathrm{~Hz}, 1 \mathrm{H})$, $5.14(\mathrm{~d}, J=9.6 \mathrm{~Hz}, 1 \mathrm{H}), 4.85$ (ddd, $J=10.8,7.2,1.8 \mathrm{~Hz}, 1 \mathrm{H}), 4.78(\mathrm{~d}, J=4.2 \mathrm{~Hz}, 1 \mathrm{H}), 4.68(\mathrm{~d}$, 
$J=4.8 \mathrm{~Hz}, 1 \mathrm{H}), 4.60(\mathrm{~d}, J=3.6 \mathrm{~Hz}, 1 \mathrm{H}), 3.97$ (bs, $1 \mathrm{H}), 3.82(\mathrm{bs}, 1 \mathrm{H}), 3.09(\mathrm{~m}, 1 \mathrm{H}), 2.69(\mathrm{~m}$, $1 \mathrm{H}), 2.12(\mathrm{~s}, 3 \mathrm{H}), 2.20-2.00(\mathrm{~m}, 4 \mathrm{H}), 1.90(\mathrm{~m}, 2 \mathrm{H}), 1.83(\mathrm{~s}, 3 \mathrm{H}), 1.71(\mathrm{~s}, 3 \mathrm{H}), 1.61(\mathrm{~s}, 3 \mathrm{H})$, 1.52-1.40 (m, 2 H), 1.36-1.20 (m, 2 H), 1.09 (m, 1 H), 0.90 (d, $J=6.6 \mathrm{~Hz}, 3 \mathrm{H}), 0.85$ ppm (m, 1 $\mathrm{H}) ;{ }^{13} \mathrm{C}$ NMR $\left(150 \mathrm{MHz}, \mathrm{DMSO}-d^{6}\right): \delta=166.2,149.5,148.8,135.2,135.1,134.7,132.0,131.3$, $129.8,127.8,127.4,121.4,75.5,74.3,74.0,74.0,72.2,43.3,37.3,36.5,32.5,28.4,24.2,16.9$, 16.4, 12.4, 8.7 ppm; HRMS (ESI): calcd for $\mathrm{C}_{32} \mathrm{H}_{47} \mathrm{NO}_{6} \mathrm{Na}^{+}\left[\mathrm{M}+\mathrm{Na}^{+}\right]$564.3296, found 564.3284 .

Macrocycle 89: To a solution of cyclic carbonate 86 (16.0 mg, $25.0 \mu \mathrm{mol})$ in $\mathrm{CH}_{2} \mathrm{Cl}_{2}(5.0 \mathrm{~mL}$, $0.005 \mathrm{M})$ was added Grubbs II catalyst $(3.6 \mathrm{mg}, 5.0 \mu \mathrm{mol})$ at room temperature. The reaction

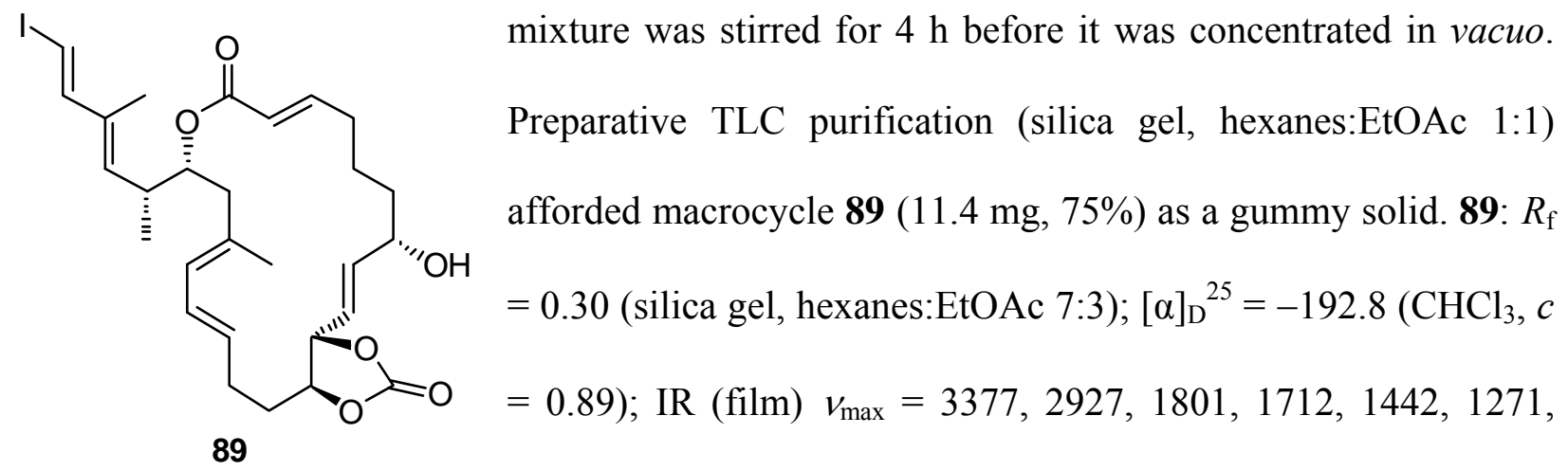
1178, 1049, $773 \mathrm{~cm}^{-1} ;{ }^{1} \mathrm{H}$ NMR $\left(600 \mathrm{MHz}, \mathrm{CDCl}_{3}\right): \delta=7.07(\mathrm{~d}, J=14.6 \mathrm{~Hz}, 1 \mathrm{H}), 6.84(\mathrm{dt}, J=$ 15.6, $6.9 \mathrm{~Hz}, 1 \mathrm{H}), 6.23(\mathrm{~d}, J=14.6 \mathrm{~Hz}, 1 \mathrm{H}), 6.24(\mathrm{~m}, 2 \mathrm{H}), 5.95(\mathrm{dd}, J=15.5,3.6 \mathrm{~Hz}, 1 \mathrm{H})$, $5.83(\mathrm{ddd}, J=15.5,8.0,1.7 \mathrm{~Hz}, 1 \mathrm{H}), 5.74(\mathrm{dt}, J=15.6,1.4 \mathrm{~Hz}, 1 \mathrm{H}), 5.31(\mathrm{~d}, J=10.9 \mathrm{~Hz}, 1 \mathrm{H})$, $5.46(\mathrm{dt}, J=15.0,7.5 \mathrm{~Hz}, 1 \mathrm{H}), 5.31(\mathrm{~d}, J=10.0 \mathrm{~Hz}, 1 \mathrm{H}), 5.00(\mathrm{ddd}, J=11.0,7.6,2.1 \mathrm{~Hz}, 1 \mathrm{H})$, $4.68(\mathrm{~m}, 1 \mathrm{H}), 4.41-4.35(\mathrm{~m}, 2 \mathrm{H}), 2.77-2.72(\mathrm{~m}, 1 \mathrm{H}), 2.39-2.30(\mathrm{~m}, 1 \mathrm{H}), 2.30-2.10(\mathrm{~m}, 6 \mathrm{H})$, 2.03-1.96 (m, $1 \mathrm{H}), 1.75(\mathrm{~d}, J=1.2 \mathrm{~Hz}, 3 \mathrm{H}), 1.72(\mathrm{~s}, 3 \mathrm{H}), 1.70-1.40(\mathrm{~m}, 4 \mathrm{H}), 1.00 \mathrm{ppm}(\mathrm{d}, J=$ $6.6 \mathrm{~Hz}, 3 \mathrm{H}) ;{ }^{13} \mathrm{C}$ NMR $\left(150 \mathrm{MHz}, \mathrm{CDCl}_{3}\right): \delta=166.0,154.2,149.5,148.2,140.3,135.2,135.0$, $133.4,129.3,128.0,127.3,123.3,121.8,82.4,80.2,74.4,74.0,70.2,43.0,37.3,35.4,33.4,32.3$, 
27.2, 22.8, 16.9, 16.6, 12.4 ppm; HRMS (ESI): calcd for $\mathrm{C}_{28} \mathrm{H}_{37} \mathrm{IO}_{6} \mathrm{Na}^{+}\left[\mathrm{M}+\mathrm{Na}^{+}\right]$619.1527, found 619.1519 .

Triol 90: A solution of cyclic carbonate $89(11.4 \mathrm{mg}, 19.1 \mu \mathrm{mol})$ in a mixture of $\mathrm{Et}_{3} \mathrm{~N} / \mathrm{MeOH} / \mathrm{H}_{2} \mathrm{O}(1: 5: 1,2 \mathrm{~mL})$ was heated at $40{ }^{\circ} \mathrm{C}$ for $6 \mathrm{~h}$. The reaction mixture was cooled to

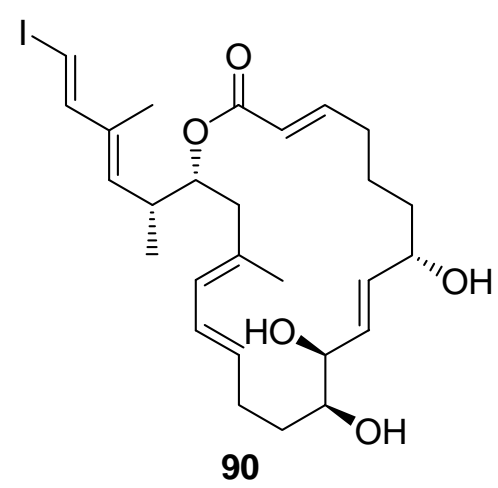
room temperature before it was concentrated in vacuo. Preparative TLC purification (silica gel, hexanes:EtOAc 1:9) afforded triol $90(7.1 \mathrm{mg}, 65 \%)$ as a gummy solid. $90: R_{\mathrm{f}}=0.46$ (silica gel, hexanes:EtOAc $0: 1) ;[\alpha]_{\mathrm{D}}{ }^{25}=-28.5\left(\mathrm{CHCl}_{3}, c=1.00\right)$; IR (film) $v_{\max } 3361,2927,1715,1654,1261,1185,1016,754$ $\mathrm{cm}^{-1} ;{ }^{1} \mathrm{H} \mathrm{NMR}\left(600 \mathrm{MHz}, \mathrm{CDCl}_{3}\right): \delta=7.06(\mathrm{~d}, J=14.6 \mathrm{~Hz}, 1 \mathrm{H})$, $6.85(\mathrm{ddd}, J=14.9,8.9,5.5 \mathrm{~Hz}, 1 \mathrm{H}), 6.23(\mathrm{~d}, J=5.9 \mathrm{~Hz}, 1 \mathrm{H}), 6.17(\mathrm{dd}, J=14.6,10.7 \mathrm{~Hz}, 1 \mathrm{H})$, $5.78(\mathrm{bm}, 1 \mathrm{H}), 5.68(\mathrm{~d}, J=10.4 \mathrm{~Hz}, 1 \mathrm{H}), 5.53(\mathrm{ddd}, J=14.6,9.2,4.9 \mathrm{~Hz}, 1 \mathrm{H}), 5.31(\mathrm{~d}, J=9.9$ Hz, $1 \mathrm{H}), 5.00$ (dt, $J=9.9,1.7 \mathrm{~Hz}, 1 \mathrm{H}), 4.15$ (brd, $1 \mathrm{H}$ ), 3.99 (brs, $1 \mathrm{H}), 3.50$ (brs, $1 \mathrm{H}), 3.14$ (brd, $J=3.6 \mathrm{~Hz}, 1 \mathrm{H}), 2.72(\mathrm{~m}, 1 \mathrm{H}), 2.35-2.05(\mathrm{~m}, 8 \mathrm{H}), 1.75(\mathrm{~s}, 3 \mathrm{H}), 1.71(\mathrm{~s}, 3 \mathrm{H}), 1.54-1.04$ (m, $8 \mathrm{H}), 0.99 \mathrm{ppm}(\mathrm{d}, J=7.2 \mathrm{~Hz}, 3 \mathrm{H}) ;{ }^{13} \mathrm{C}$ NMR $\left(150 \mathrm{MHz}, \mathrm{CDCl}_{3}\right): \delta=166.2,149.5,148.8$, 135.2, 135.1, 134.7, 132.0, 131.3, 129.8, 127.8, 127.4, 121.4, 74.3, 74.0, 74.0, 72.2, 46.2, 43.3, 37.3, 36.5, 32.5, 28.4, 24.2, 16.9, 16.4, 12.4, 8.7 ppm; HRMS (ESI): calcd for $\mathrm{C}_{27} \mathrm{H}_{39} \mathrm{IO}_{5} \mathrm{Na}^{+}{ }^{+} \mathrm{M}$ $\left.+\mathrm{Na}^{+}\right]$593.1734, found 593.1722.

Enamide 88: To a mixture of vinyl iodide 90 (6.4 mg, $11.2 \mu \mathrm{mol})$, amide 42 (2.2 $\mathrm{mg}, 22.4$ $\mu \mathrm{mol}), \mathrm{K}_{2} \mathrm{CO}_{3}(8.0 \mathrm{mg}, 56.1 \mu \mathrm{mol})$ and $\mathrm{CuI}(3.2 \mathrm{mg}, 16.8 \mu \mathrm{mol})$ in DMF $(2.0 \mathrm{~mL})$ was added $N, N^{\prime}$-dimethyethylenediamine $(5.8 \mu \mathrm{L}, 52.6 \mu \mathrm{mol})$ at room temperature. The reaction mixture 


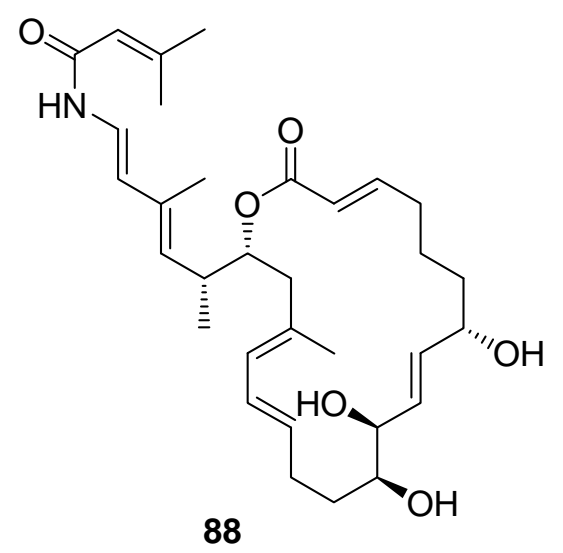

was stirred for $1 \mathrm{~h}$ before it was concentrated in vacuo. The resulting residue was diluted with EtOAc $(10 \mathrm{~mL})$, filtered through a pad of Celite ${ }^{\circledR}$, washed with EtOAc $(10 \mathrm{~mL})$ and concentrated in vacuo. Preparative TLC purification (silica gel, EtOAc: $\left.\mathrm{Et}_{3} \mathrm{~N} 100: 1\right)$ afforded enamide $88(3.1 \mathrm{mg}, 51 \%)$ as a colorless oil. All physical properties of this compound were identical to those reported for decarbamated product $\mathbf{8 8}$ obtained from vinyl iodide $\mathbf{8 7}$.

Enamide 91: To a solution of vinyl iodide 85 (10.0 mg, $0.0156 \mathrm{mmol})$, amide 42 (3.1 $\mathrm{mg}, 0.031$ mmol), $\mathrm{CuI}(3.0 \mathrm{mg}, 0.0156 \mathrm{mmol})$ and $\mathrm{K}_{2} \mathrm{CO}_{3}(10.8 \mathrm{mg}, 0.078 \mathrm{mmol}$, flame dried) in DMF (1.5

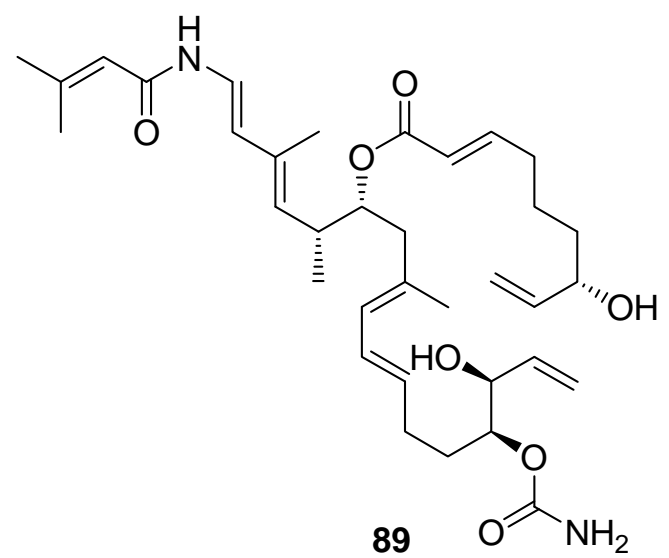
$\mathrm{mL})$ was added $N, N^{\prime}$-dimethylethylenediamine $(5.0 \mu \mathrm{L}$, $0.047 \mathrm{mmol}$ ) at room temperature. The reaction mixture was stirred for $1 \mathrm{~h}$ before it was diluted with EtOAc (20 $\mathrm{mL})$, filtered through a short pad of Celite ${ }^{\circledR}$ and concentrated in vacuo. Preparative TLC purification (silica gel, $\mathrm{CH}_{2} \mathrm{Cl}_{2}$ :acetone 7:2) afforded enamide 91 $(4.4 \mathrm{mg}, 46 \%)$ as a colorless oil. 91: $R_{\mathrm{f}}=0.47$ (silica gel, hexanes:EtOAc $\left.5: 2\right) ;[\alpha]_{\mathrm{D}}{ }^{25}=+21.5$ $(\mathrm{MeOH}, c=0.20)$; IR (film) $v_{\max } 3348,2926,1711,1640,1520,1388,1276,1262,1192,1051$, $926 \mathrm{~cm}^{-1} ;{ }^{1} \mathrm{H}$ NMR $\left(600 \mathrm{MHz}, \mathrm{DMSO}-d^{6}\right): \delta=9.83(\mathrm{~d}, J=10.2 \mathrm{~Hz}, 1 \mathrm{H}), 6.82(\mathrm{dd}, J=14.7$, $10.5 \mathrm{~Hz}, 1 \mathrm{H}), 6.80$ (ddd, $J=15.6,11.4,5.7 \mathrm{~Hz}, 1 \mathrm{H}), 6.43$ (brs, $2 \mathrm{H}), 6.13$ (dd, $J=15.0,10.8 \mathrm{~Hz}$, $1 \mathrm{H}), 5.82(\mathrm{~d}, J=14.4 \mathrm{~Hz}, 1 \mathrm{H}), 5.79(\mathrm{ddd}, J=17.4,10.8,5.4 \mathrm{~Hz}, 1 \mathrm{H}), 5.77(\mathrm{~d}, J=15.6 \mathrm{~Hz}, 1$ H), $5.75(\mathrm{ddd}, J=16.8,10.8,4.8 \mathrm{~Hz}, 1 \mathrm{H}), 5.67(\mathrm{~s}, 1 \mathrm{H}), 5.66(\mathrm{~d}, J=9.6 \mathrm{~Hz}, 1 \mathrm{H}), 5.48(\mathrm{dt}, J=$ 15.0, $6.9 \mathrm{~Hz}, 1 \mathrm{H}), 5.20$ (brd, $J=17.4 \mathrm{~Hz}, 1 \mathrm{H}), 5.14$ (d, $J=9.6 \mathrm{~Hz}, 1 \mathrm{H}), 5.11$ (brd, $J=17.4 \mathrm{~Hz}$, 
$1 \mathrm{H}), 5.07$ (brd, $J=10.8 \mathrm{~Hz}, 1 \mathrm{H}), 5.00$ (d, $J=5.4 \mathrm{~Hz}, 1 \mathrm{H}), 4.96$ (brd, $J=10.2 \mathrm{~Hz}, 1 \mathrm{H}), 4.89$ $(\mathrm{dt}, J=8.4,4.5 \mathrm{~Hz}, 1 \mathrm{H}), 4.68(\mathrm{~d}, J=4.8 \mathrm{~Hz}, 1 \mathrm{H}), 4.52(\mathrm{dt}, J=9.6,4.4 \mathrm{~Hz}, 1 \mathrm{H}), 4.01(\mathrm{q}, J=$ $5.0 \mathrm{~Hz}, 1 \mathrm{H}), 3.89$ (t, $J=5.4 \mathrm{~Hz}, 1 \mathrm{H}), 2.70(\mathrm{dqd}, J=10.2,7.2,6.6 \mathrm{~Hz}, 1 \mathrm{H}), 2.20-1.98(\mathrm{~m}, 4 \mathrm{H})$, 2.10 (s, $3 \mathrm{H}), 1.81$ (s, $3 \mathrm{H}), 1.67$ (s, $3 \mathrm{H}), 1.63(\mathrm{~s}, 3 \mathrm{H}), 1.60-1.54$ (m, $2 \mathrm{H}), 1.47-1.32(\mathrm{~m}, 4 \mathrm{H})$, $1.28-1.18(\mathrm{~m}, 2 \mathrm{H}), 0.89 \mathrm{ppm}(\mathrm{d}, J=6.6 \mathrm{~Hz}, 3 \mathrm{H}) ;{ }^{13} \mathrm{C}$ NMR $\left(150 \mathrm{MHz}, \mathrm{DMSO}-d^{6}\right): \delta=165.8$, 163.6, 157.2, 152.1, 149.8, 143.0, 138.6, 133.3, 132.5, 132.3, 129.6, 127.8, 122.5, 121.5, 118.6, 117.0, 115.6, 113.5, 75.1, 72.2, 71.1, 42.3, 36.8, 36.3, 31.8, 29.7, 28.9, 27.4, 24.0, 20.0, 17.6, 16.9, 13.0 ppm; HRMS (ESI): calcd for $\mathrm{C}_{35} \mathrm{H}_{52} \mathrm{~N}_{2} \mathrm{O}_{7} \mathrm{Na}^{+}\left[\mathrm{M}+\mathrm{Na}^{+}\right]$635.3667, found 635.3647.

Palmerolide A isomer ent-(19-epi-20-epi-1): To a solution of enamide 89 (2.0 mg, 0.0033 mmol) in $\mathrm{CH}_{2} \mathrm{Cl}_{2}(1.6 \mathrm{~mL})$ was added Grubbs II catalyst $(0.56 \mathrm{mg}, 0.67 \mu \mathrm{mol})$ at room

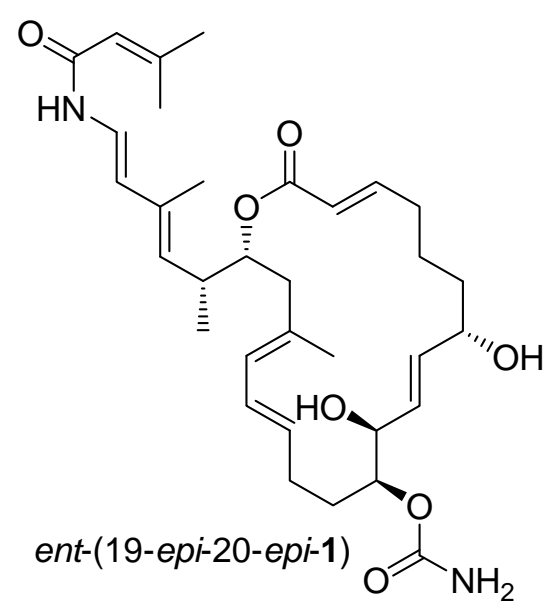
temperature. The reaction mixture was stirred for $2 \mathrm{~h}$ before it was concentrated in vacuo. Preparative TLC purification (silica gel, hexanes:EtOAc 0:1) afforded ent-(19-epi-20-epi-1) $(1.4 \mathrm{mg}, 73 \%)$ as a colorless oil. All physical properties of this compound were identical to those reported for ent-(19-epi-20epi-1) obtained from vinyl iodide $\mathbf{8 7 .}$

PMB ether 92: To a solution of $\mathrm{NaH}(340 \mathrm{mg}, 8.5 \mathrm{mmol})$ in DMF $(20 \mathrm{~mL})$ were added a solution of alcohol $21(1.20 \mathrm{~g}, 6.5 \mathrm{mmol})$ in DMF $(8 \mathrm{~mL})$ and $\mathrm{PMBCl}(1.0 \mathrm{~mL}, 7.8 \mathrm{mmol})$ at 0 ${ }^{\circ} \mathrm{C}$. The reaction mixture was stirred at room temperature for $5 \mathrm{~h}$ before it was
92 
$(3 \times 50 \mathrm{~mL})$, and the combined organic layers were dried $\left(\mathrm{Na}_{2} \mathrm{SO}_{4}\right)$ and concentrated in vacuo. Flash column chromatography (silica gel, hexanes:EtOAc 5:1) afforded PMB ether 92 (1.82 g, 91\%) as a colorless oil. 92: $R_{\mathrm{f}}=0.52$ (silica gel, hexanes:EtOAc 5:1); $[\alpha]_{\mathrm{D}}{ }^{25}=+9.0\left(\mathrm{CH}_{2} \mathrm{Cl}_{2, c}=\right.$ 4.0); IR (film) $v_{\max } 3294,2934,2838,2117,1612,1514,1247,1031 \mathrm{~cm}^{-1}$; ${ }^{1} \mathrm{H}$ NMR (600 MHz, $\left.\mathrm{CDCl}_{3}\right): \delta=7.30(\mathrm{~d}, J=9.0 \mathrm{~Hz}, 2 \mathrm{H}), 6.89(\mathrm{~d}, J=9.0 \mathrm{~Hz}, 2 \mathrm{H}), 5.81(\mathrm{ddd}, J=17.4,10.2,7.2 \mathrm{~Hz}$, $1 \mathrm{H}), 5.32(\mathrm{brd}, J=16.8 \mathrm{~Hz}, 1 \mathrm{H}), 5.30(\mathrm{brd}, J=10.2 \mathrm{~Hz}, 1 \mathrm{H}), 4.73(\mathrm{~d}, J=6.6 \mathrm{~Hz}, 1 \mathrm{H}), 4.71(\mathrm{~d}$, $J=10.8 \mathrm{~Hz}, 1 \mathrm{H}), 4.62(\mathrm{~d}, J=6.6 \mathrm{~Hz}, 1 \mathrm{H}), 4.55(\mathrm{~d}, J=11.4 \mathrm{~Hz}, 1 \mathrm{H}), 4.18(\mathrm{t}, J=6.3 \mathrm{~Hz}, 1 \mathrm{H})$, $3.82(\mathrm{~s}, 3 \mathrm{H}), 3.65(\mathrm{ddd}, J=9.0,5.4,3.0 \mathrm{~Hz}, 1 \mathrm{H}), 3.40(\mathrm{~s}, 3 \mathrm{H}), 2.34-2.28(\mathrm{~m}, 2 \mathrm{H}), 1.96(\mathrm{t}, J=$ $2.7 \mathrm{~Hz}, 1 \mathrm{H}), 1.81$ (dddd, $J=13.8,11.4,8.4,3.0 \mathrm{~Hz}, 1 \mathrm{H}), 1.66 \mathrm{ppm}(\mathrm{dddd}, J=13.8,9.0,7.2,5.4$ $\mathrm{Hz}, 1 \mathrm{H}) ;{ }^{13} \mathrm{C} \mathrm{NMR}\left(150 \mathrm{MHz}, \mathrm{CDCl}_{3}\right): \delta=159.3,134.7,130.7,129.7,118.7,113.8,94.3,84.2$ 79.2, 78.6, 73.2, 68.6, 55.5, 55.3, 29.7, 14.8 ppm; HRMS (ESI): calcd for $\mathrm{C}_{18} \mathrm{H}_{24} \mathrm{O}_{4} \mathrm{Na}^{+}[\mathrm{M}+$ $\left.\mathrm{Na}^{+}\right]$327.1567, found 327.1564 .

TMS alkyne 93: To a solution of alkyne $92(8.117 \mathrm{~g}, 26.7 \mathrm{mmol})$ in THF $(90 \mathrm{~mL})$ was added $n$ $\operatorname{BuLi}(1.6 \mathrm{M}$ in hexanes, $23 \mathrm{~mL}, 36.8 \mathrm{mmol})$ at $-78^{\circ} \mathrm{C}$. The reaction mixture was stirred for $1 \mathrm{~h}$ before $\mathrm{TMSCl}(4.1 \mathrm{~mL}, 32.0 \mathrm{mmol})$ was added at $-78^{\circ} \mathrm{C}$ and stirred
for further $30 \mathrm{~min}$. The reaction mixture was warmed to room $\mathrm{mL})$. The resulting mixture was extracted with $\mathrm{Et}_{2} \mathrm{O}(3 \times 50 \mathrm{~mL})$, and the combined organic layers were dried $\left(\mathrm{Na}_{2} \mathrm{SO}_{4}\right)$ and concentrated in vacuo. Flash column chromatography (silica gel, hexanes:EtOAc 6:1) afforded TMS alkyne 93 (9.2 g, 92\%) as a colorless oil. 93: $R_{\mathrm{f}}=0.57$ (silica gel, hexanes:EtOAc 5:1); $[\alpha]_{\mathrm{D}}^{25}=+13.9\left(\mathrm{CH}_{2} \mathrm{Cl}_{2}, c=2.3\right)$; IR (film) $v_{\max } 3005,2962,2837$, $2174,1613,1513,1276,1260,1034,842 \mathrm{~cm}^{-1} ;{ }^{1} \mathrm{H}$ NMR $\left(600 \mathrm{MHz}, \mathrm{CDCl}_{3}\right): \delta=7.30(\mathrm{~d}, J=8.4$ 
$\mathrm{Hz}, 2 \mathrm{H}), 6.88$ (d, $J=8.4 \mathrm{~Hz}, 2 \mathrm{H}), 5.81$ (ddd, $J=17.4,10.8,7.2 \mathrm{~Hz}, 1 \mathrm{H}), 5.32$ (brd, $J=17.4 \mathrm{~Hz}$, $1 \mathrm{H}), 5.29$ (brd, $J=10.2 \mathrm{~Hz}, 1 \mathrm{H}), 4.72(\mathrm{~d}, J=6.6 \mathrm{~Hz}, 1 \mathrm{H}), 4.70(\mathrm{~d}, J=10.8 \mathrm{~Hz}, 1 \mathrm{H}), 4.62(\mathrm{~d}, J$ $=6.6 \mathrm{~Hz}, 1 \mathrm{H}), 4.55(\mathrm{~d}, J=10.8 \mathrm{~Hz}, 1 \mathrm{H}), 4.17(\mathrm{dd}, J=7.2,6.0 \mathrm{~Hz}, 1 \mathrm{H}), 3.82(\mathrm{~s}, 3 \mathrm{H}), 3.65$ (ddd, $J=9.6,5.4,4.2 \mathrm{~Hz}, 1 \mathrm{H}), 3.39$ (s, $3 \mathrm{H}), 2.34$ (dddd, $J=11.4,9.6,7.8,5.4 \mathrm{~Hz}, 2 \mathrm{H}), 1.79$ (dddd, $J=13.8,11.4,7.8,3.6 \mathrm{~Hz}, 1 \mathrm{H}), 1.67$ (dddd, $J=14.4,9.6,7.2,5.4 \mathrm{~Hz}, 1 \mathrm{H}), 0.16 \mathrm{ppm}(\mathrm{s}$, $9 \mathrm{H}) ;{ }^{13} \mathrm{C}$ NMR $\left(150 \mathrm{MHz}, \mathrm{CDCl}_{3}\right): \delta=159.2,134.8,130.7,129.6,118.4,113.7,107.1,94.3$, 84.8, 79.2, 78.4, 73.1, 55.5, 55.2, 29.6, 16.2, 0.15 ppm; HRMS (ESI): calcd for $\mathrm{C}_{21} \mathrm{H}_{32} \mathrm{O}_{4} \mathrm{SiNa}^{+}$ $\left[\mathrm{M}+\mathrm{Na}^{+}\right]$399.1962, found 399.1962.

Alcohol 94: To a solution of MOM ether $93(7.4 \mathrm{~g}, 19.7 \mathrm{mmol})$ in $i$-PrOH $(100 \mathrm{~mL})$ was added $\mathrm{CBr}_{4}(1.30 \mathrm{~g}, 3.92 \mathrm{mmol})$ at room temperature. The reaction mixture was heated at $90{ }^{\circ} \mathrm{C}$ for $2 \mathrm{~h}$ (silica gel, hexanes:EtOAc 9:2) afforded alcohol $94(4.5 \mathrm{~g}, 69 \%)$ as a
colorless oil. 94: $R_{\mathrm{f}}=0.25$ (silica gel, hexanes:EtOAc 5:1); $[\alpha]_{\mathrm{D}}{ }^{25}=$ $+24.3\left(\mathrm{CH}_{2} \mathrm{Cl}_{2}, c=1.7\right)$; IR (film) $v_{\max } 3448,3005,2957,2837,2174,1613,1514,1247,1173$, 1035, $839 \mathrm{~cm}^{-1} ;{ }^{1} \mathrm{H}$ NMR $\left(600 \mathrm{MHz}, \mathrm{CDCl}_{3}\right): \delta=7.29(\mathrm{~d}, J=8.4 \mathrm{~Hz}, 2 \mathrm{H}), 6.90(\mathrm{~d}, J=8.4 \mathrm{~Hz}$, $2 \mathrm{H}), 5.91$ (ddd, $J=16.8,10.2,6.0 \mathrm{~Hz}, 1 \mathrm{H}), 5.38$ (brd, $J=17.4 \mathrm{~Hz}, 1 \mathrm{H}), 5.28(\mathrm{brd}, J=10.2 \mathrm{~Hz}$, $1 \mathrm{H}), 4.62(\mathrm{~d}, J=10.8 \mathrm{~Hz}, 1 \mathrm{H}), 4.55(\mathrm{~d}, J=10.8 \mathrm{~Hz}, 1 \mathrm{H}), 4.08(\mathrm{td}, J=6.0,4.8 \mathrm{~Hz}, 1 \mathrm{H}), 3.82(\mathrm{~s}$, $3 \mathrm{H}$ ), 3.55 (ddd, $J=7.2,5.4,4.8 \mathrm{~Hz}, 1 \mathrm{H}), 2.38-2.35$ (m, $2 \mathrm{H}), 2.34$ (brs, $1 \mathrm{H}), 1.84-1.76$ (m, 2 H), $0.17 \mathrm{ppm}(\mathrm{s}, 9 \mathrm{H}) ;{ }^{13} \mathrm{C} \mathrm{NMR}\left(150 \mathrm{MHz}, \mathrm{CDCl}_{3}\right): \delta=159.4,137.7,130.3,129.6,116.7,113.9$, 106.9, 85.2, 80.5, 74.4, 73.0, 55.2, 29.8, 16.0, 0.15 ppm; HRMS (ESI): calcd for $\mathrm{C}_{19} \mathrm{H}_{28} \mathrm{O}_{3} \mathrm{SiNa}^{+}$ $\left[\mathrm{M}+\mathrm{Na}^{+}\right] 355.1700$, found 355.1700 . 
Enyne 95: To a solution of allylic alcohol 94 (166 mg, $0.5 \mathrm{mmol})$ and allylic alcohol 27 (244 mg, $1.0 \mathrm{mmol})$ in $\mathrm{CH}_{2} \mathrm{Cl}_{2}(10 \mathrm{~mL}$, degassed) was added Grubbs II catalyst $(42 \mathrm{mg}, 0.05 \mathrm{mmol})$ at

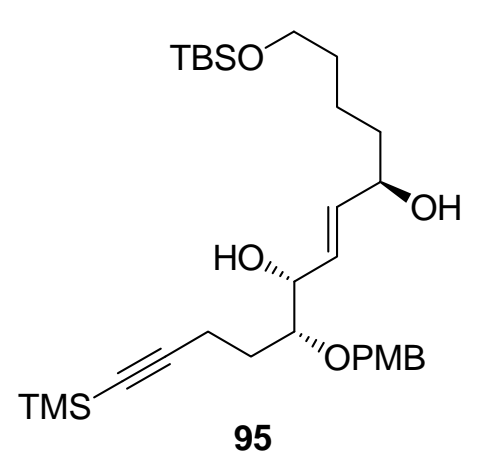
room temperature. The reaction mixture was stirred for $12 \mathrm{~h}$ before it was concentrated in vacuo. Flash column chromatography (silica gel, hexanes:EtOAc 5:2) afforded enyne 95 (112 $\mathrm{mg}, 41 \%)$ as a brown oil. 95: $R_{\mathrm{f}}=0.50$ (silica gel, hexanes:EtOAc 2:1); $[\alpha]_{\mathrm{D}}^{25}=$ $+6.1\left(\mathrm{CH}_{2} \mathrm{Cl}_{2, c}=0.50\right)$; IR (film) $v_{\max } 3390,2930,2858,2174$, $1613,1514,1249,1174,1098,839 \mathrm{~cm}^{-1} ;{ }^{1} \mathrm{H}$ NMR (600 MHz, $\left.\mathrm{CDCl}_{3}\right): \delta=7.28(\mathrm{~d}, J=8.4 \mathrm{~Hz}, 2$ H), $6.89(\mathrm{~d}, J=8.4 \mathrm{~Hz}, 2 \mathrm{H}), 5.82(\mathrm{dd}, J=15.6,6.0 \mathrm{~Hz}, 1 \mathrm{H}), 5.71(\mathrm{dd}, J=15.6,6.0 \mathrm{~Hz}, 1 \mathrm{H})$, $4.61(\mathrm{~d}, J=10.8 \mathrm{~Hz}, 1 \mathrm{H}), 4.53(\mathrm{~d}, J=11.4 \mathrm{~Hz}, 1 \mathrm{H}), 4.14(\mathrm{q}, J=4.8 \mathrm{~Hz}, 1 \mathrm{H}), 4.09$ (q, $J=5.4$ $\mathrm{Hz}, 1 \mathrm{H}), 3.81(\mathrm{~s}, 3 \mathrm{H}), 3.62(\mathrm{t}, J=6.3 \mathrm{~Hz}, 2 \mathrm{H}), 3.52(\mathrm{dt}, J=7.2,5.1 \mathrm{~Hz}, 1 \mathrm{H}), 2.48(\mathrm{~d}, J=4.8$ $\mathrm{Hz}, 1 \mathrm{H}), 2.41-2.32(\mathrm{~m}, 2 \mathrm{H}), 1.84(\mathrm{~d}, J=4.2 \mathrm{~Hz}, 1 \mathrm{H}), 1.85-1.73(\mathrm{~m}, 3 \mathrm{H}), 1.57-1.38(\mathrm{~m}, 5 \mathrm{H})$, 0.90 (s, $9 \mathrm{H}), 0.17$ (s, $9 \mathrm{H}), 0.06 \mathrm{ppm}(\mathrm{s}, 6 \mathrm{H}) ;{ }^{13} \mathrm{C} \mathrm{NMR}\left(150 \mathrm{MHz}, \mathrm{CDCl}_{3}\right): \delta=159.4,135.8$, $130.2,129.7,129.6,113.9,106.8,85.3,80.6,73.5,72.9,72.1,63.1,55.3,36.9,32.6,29.7,26.0$, 21.7, 18.4, 16.0, 0.2, -5.3 ppm; HRMS (ESI): calcd for $\mathrm{C}_{30} \mathrm{H}_{52} \mathrm{O}_{5} \mathrm{Si}_{2} \mathrm{Na}^{+}\left[\mathrm{M}+\mathrm{Na}^{+}\right] 571.3246$, found 571.3258 .

Bis-TBDPS ether 96: To a solution of secondary diol 95 (500 mg, $0.91 \mathrm{mmol})$ in DMF (3 mL) were added imidazole $(495 \mathrm{mg}, 7.28 \mathrm{mmol})$ and TBDPSCl $(0.6 \mathrm{~mL}, 2.28 \mathrm{mmol})$ at room temperature. The reaction mixture was stirred for $8 \mathrm{~h}$ before it was quenched with $\mathrm{H}_{2} \mathrm{O}(10 \mathrm{~mL})$. The resulting mixture was extracted with $\mathrm{Et}_{2} \mathrm{O}(3 \times 30 \mathrm{~mL})$, and the combined organic layers were dried $\left(\mathrm{Na}_{2} \mathrm{SO}_{4}\right)$ and concentrated in vacuo. Flash column chromatography (silica gel, hexanes:EtOAc 10:1) afforded bis-TBDPS ether $96(780 \mathrm{mg}, 84 \%)$ as a colorless oil. 96: $R_{\mathrm{f}}=$ 


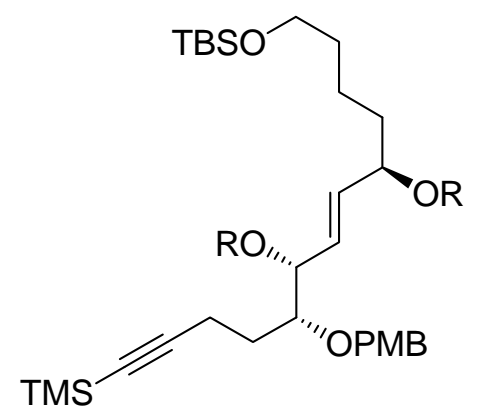

96: $\mathrm{R}=\mathrm{TBDPS} \quad=8.4 \mathrm{~Hz}, 2 \mathrm{H}), 5.64(\mathrm{dd}, J=15.6,6.0 \mathrm{~Hz}, 1 \mathrm{H}), 5.50(\mathrm{dd}, J=15.6$,

$5.4 \mathrm{~Hz}, 1 \mathrm{H}), 4.37$ (t, $J=4.2 \mathrm{~Hz}, 1 \mathrm{H}), 4.15$ (q, $J=5.4 \mathrm{~Hz}, 1 \mathrm{H}), 4.07$ (d, $J=11.4 \mathrm{~Hz}, 1 \mathrm{H}), 3.92$ $(\mathrm{d}, J=11.4 \mathrm{~Hz}, 1 \mathrm{H}), 3.79(\mathrm{~s}, 3 \mathrm{H}), 3.44$ (t, $J=6.6 \mathrm{~Hz}, 2 \mathrm{H}), 3.27(\mathrm{ddd}, J=7.2,4.2,3.0 \mathrm{~Hz}, 1 \mathrm{H})$, $2.29(\mathrm{ddd}, J=13.8,7.8,4.8 \mathrm{~Hz}, 1 \mathrm{H}), 2.17(\mathrm{dt}, J=8.4,8.4 \mathrm{~Hz}, 1 \mathrm{H}), 1.87-1.80(\mathrm{~m}, 1 \mathrm{H})$, 1.45-1.39 (m, $1 \mathrm{H}), 1.37-1.28(\mathrm{~m}, 5 \mathrm{H}), 1.24-1.17(\mathrm{~m}, 1 \mathrm{H}), 1.08$ (s, $9 \mathrm{H}), 1.04(\mathrm{~s}, 9 \mathrm{H}), 0.89$ (s, $9 \mathrm{H}), 0.17$ (s, $9 \mathrm{H}), 0.03 \mathrm{ppm}(\mathrm{s}, 6 \mathrm{H}) ;{ }^{13} \mathrm{C} \mathrm{NMR}\left(150 \mathrm{MHz}, \mathrm{CDCl}_{3}\right): \delta=158.9,136.0,136.0$, $136.0,135.9,134.5,134.3,134.2,134.0,133.8,131.0,129.8,129.6,129.5,129.3,129.1,128.0$, $127.6,127.5,127.4,127.4,113.6,107.6,84.1,80.5,73.5,72.5,71.6,63.2,55.2,37.5,32.9,28.3$, 27.12, 27.07, 26.0, 20.6, 19.4, 19.3, 18.4, 16.5, 0.2, -5.3 ppm; HRMS (ESI): calcd for $\mathrm{C}_{62} \mathrm{H}_{88} \mathrm{O}_{5} \mathrm{Si}_{4} \mathrm{Na}^{+}\left[\mathrm{M}+\mathrm{Na}^{+}\right]$1047.5601, found 1047.5611.

Alcohol 97: To a solution of TBS ether $96(780 \mathrm{mg}, 0.761 \mathrm{mmol})$ in AcOH $(7.0 \mathrm{~mL})$ and water $(1.0 \mathrm{~mL})$ was added PTSA $(7 \mathrm{mg}, 0.037 \mathrm{mmol})$ at room temperature. The reaction mixture was

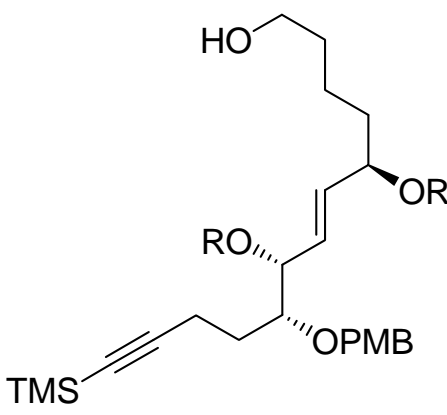

97: $\mathrm{R}=\mathrm{TBDPS}$ stirred for $3 \mathrm{~h}$ before it was concentrated in vacuo. The resulting residue was diluted with $\mathrm{Et}_{2} \mathrm{O}(80 \mathrm{~mL})$, washed with $\mathrm{NaHCO}_{3}(2 \times$ $15 \mathrm{~mL}$, sat. aq.), dried $\left(\mathrm{Na}_{2} \mathrm{SO}_{4}\right)$ and concentrated in vacuo. Flash column chromatography (silica gel, hexanes:EtOAc 4:1) afforded alcohol $97(531 \mathrm{mg}, 77 \%)$ as a colorless oil. 97: $R_{\mathrm{f}}=0.40$ (silica gel, hexanes:EtOAc 4:1); $[\alpha]_{\mathrm{D}}{ }^{25}=+30.9\left(\mathrm{CH}_{2} \mathrm{Cl}_{2}, c=2.95\right)$; IR (film) 
$V_{\max } 3371,2955,2929,2857,2174,1613,1589,1514,1248,1110,841,822 \mathrm{~cm}^{-1} ;{ }^{1} \mathrm{H}$ NMR $(600$ $\left.\mathrm{MHz}, \mathrm{CDCl}_{3}\right): \delta=7.71-7.62(\mathrm{~m}, 8 \mathrm{H}), 7.49-7.30(\mathrm{~m}, 12 \mathrm{H}), 7.02(\mathrm{~d}, J=8.4 \mathrm{~Hz}, 2 \mathrm{H}), 6.79(\mathrm{~d}, J$ $=8.4 \mathrm{~Hz}, 2 \mathrm{H}), 5.65(\mathrm{dd}, J=15.6,6.0 \mathrm{~Hz}, 1 \mathrm{H}), 5.60(\mathrm{dd}, J=15.6,4.8 \mathrm{~Hz}, 1 \mathrm{H}), 4.41(\mathrm{t}, J=4.5$ Hz, $1 \mathrm{H}), 4.19(\mathrm{q}, J=5.4 \mathrm{~Hz}, 1 \mathrm{H}), 4.11(\mathrm{~d}, J=11.4 \mathrm{~Hz}, 1 \mathrm{H}), 3.97(\mathrm{~d}, J=11.4 \mathrm{~Hz}, 1 \mathrm{H}), 3.80(\mathrm{~s}$, $3 \mathrm{H}), 3.44$ (t, $J=6.6 \mathrm{~Hz}, 2 \mathrm{H}), 3.31$ (ddd, $J=6.6,4.2,2.4 \mathrm{~Hz}, 1 \mathrm{H}), 2.33$ (ddd, $J=12.6,7.8,4.8$ Hz, $1 \mathrm{H}), 2.19$ (dt, $J=8.4,8.4 \mathrm{~Hz}, 1 \mathrm{H}), 1.89$ (dddd, $J=13.8,10.8,7.8,2.4 \mathrm{~Hz}, 1 \mathrm{H}), 1.51-1.44$ (m, $1 \mathrm{H}), 1.37-1.29(\mathrm{~m}, 5 \mathrm{H}), 1.25-1.16(\mathrm{~m}, 1 \mathrm{H}), 1.11(\mathrm{~s}, 9 \mathrm{H}), 1.08(\mathrm{~s}, 9 \mathrm{H}), 0.19$ ppm (s, $9 \mathrm{H})$; ${ }^{13} \mathrm{C}$ NMR $\left(150 \mathrm{MHz}, \mathrm{CDCl}_{3}\right): \delta=159.0,136.0,136.0,136.0,135.9,134.6,134.3,134.1,134.0$, $133.8,130.9,129.8,129.6,129.5,129.4,129.2,128.2,127.7,127.5,127.5,127.4,113.6,107.6$ $84.2,80.6,73.4,72.5,71.7,62.7,55.2,37.2,32.6,28.4,27.1,27.1,20.4,19.4,19.3,16.5,0.3$ ppm; HRMS (ESI): calcd for $\mathrm{C}_{56} \mathrm{H}_{74} \mathrm{O}_{5} \mathrm{Si}_{3} \mathrm{Na}^{+}\left[\mathrm{M}+\mathrm{Na}^{+}\right]$933.4736, found 933.4744.

Bromoacetylene 98: To a solution of acetylene $97(60 \mathrm{mg}, 0.066 \mathrm{mmol})$ in acetone $(1.0 \mathrm{~mL})$ were added NBS $(13 \mathrm{mg}, 0.073 \mathrm{mmol})$ and $\mathrm{AgNO}_{3}(11 \mathrm{mg}, 0.066 \mathrm{mmol})$ at room temperature.

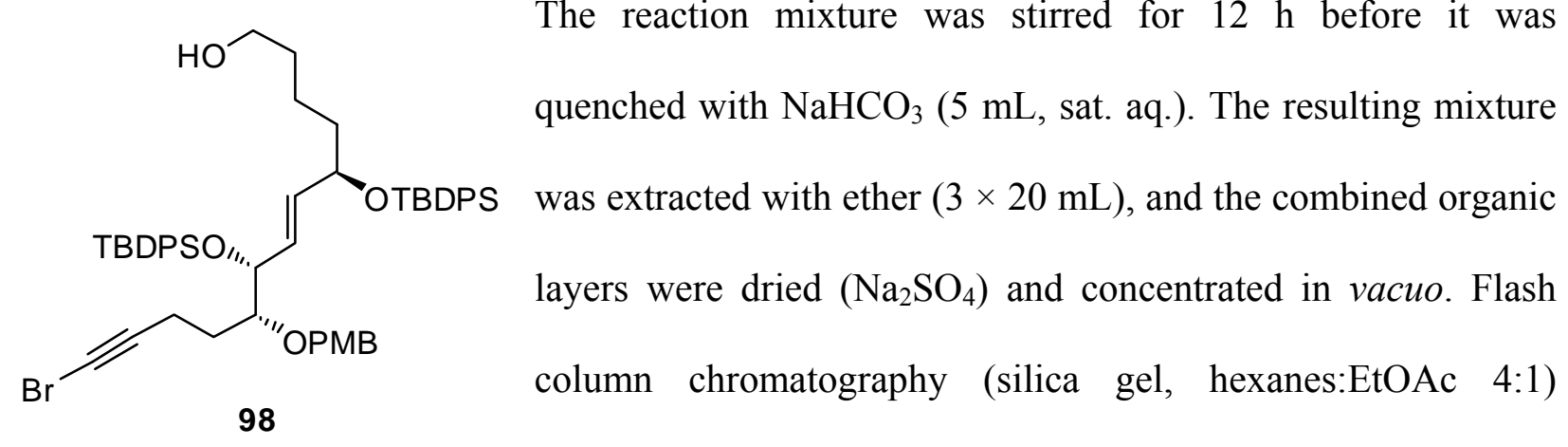

afforded bromoacetylene $98(47 \mathrm{mg}, 78 \%)$ as a colorless oil. 98: $R_{\mathrm{f}}=0.36$ (silica gel, hexanes:EtOAc 4:1); $[\alpha]_{\mathrm{D}}{ }^{25}=+52.7\left(\mathrm{CH}_{2} \mathrm{Cl}_{2, c}=1.5\right)$; IR (film) $v_{\max } 3411,2931,2857,1612$, $1513,1427,1248,1110,1054,822 \mathrm{~cm}^{-1} ;{ }^{1} \mathrm{H}$ NMR $\left(600 \mathrm{MHz}, \mathrm{CDCl}_{3}\right): \delta=7.71-7.66(\mathrm{~m}, 8 \mathrm{H})$ 7.49-7.31 (m, $12 \mathrm{H}), 6.99(\mathrm{~d}, J=8.4 \mathrm{~Hz}, 2 \mathrm{H}), 6.79(\mathrm{~d}, J=8.4 \mathrm{~Hz}, 2 \mathrm{H}), 5.72(\mathrm{dd}, J=15.6,6.0$ 
$\mathrm{Hz}, 1 \mathrm{H}), 5.62(\mathrm{dd}, J=15.6,5.4 \mathrm{~Hz}, 1 \mathrm{H}), 4.42(\mathrm{t}, J=4.5 \mathrm{~Hz}, 1 \mathrm{H}), 4.23(\mathrm{q}, J=5.8 \mathrm{~Hz}, 1 \mathrm{H})$, $4.06(\mathrm{~d}, J=11.4 \mathrm{~Hz}, 1 \mathrm{H}), 3.85(\mathrm{~d}, J=11.4 \mathrm{~Hz}, 1 \mathrm{H}), 3.80(\mathrm{~s}, 3 \mathrm{H}), 3.46(\mathrm{t}, J=6.6 \mathrm{~Hz}, 2 \mathrm{H}), 3.31$ (ddd, $J=6.6,4.2,2.4 \mathrm{~Hz}, 1 \mathrm{H}), 2.25$ (ddd, $J=12.0,6.6,1.8 \mathrm{~Hz}, 1 \mathrm{H}), 2.15$ (ddd, $J=16.2,9.6$, $6.6 \mathrm{~Hz}, 1 \mathrm{H}), 1.88-1.82$ (m, $1 \mathrm{H}), 1.62$ (brs, $1 \mathrm{H}), 1.44-1.35$ (m, $6 \mathrm{H}), 1.27-1.19$ (m, $1 \mathrm{H}), 1.12$ (s, $9 \mathrm{H}), 1.09 \mathrm{ppm}(\mathrm{s}, 9 \mathrm{H}) ;{ }^{13} \mathrm{C} \mathrm{NMR}\left(150 \mathrm{MHz}, \mathrm{CDCl}_{3}\right): \delta=159.0,136.1,136.0,136.0,135.9$, 134.6, 134.2, 134.1, 133.9, 133.7, 130.7, 129.9, 129.7, 129.6, 129.4, 129.2, 128.2, 127.8, 127.6, $127.5,127.4,113.6,80.4,80.0,73.4,72.0,71.6,62.8,55.3,37.6,37.2,32.6,28.0,27.1,20.4$, 19.4, 16.2 ppm; HRMS (ESI): calcd for $\mathrm{C}_{53} \mathrm{H}_{65} \mathrm{BrO}_{5} \mathrm{Si}_{2} \mathrm{Na}^{+}\left[\mathrm{M}+\mathrm{Na}^{+}\right]$939.3446, found 939.3461.

Enoate 99: To a solution of primary alcohol $98(50 \mathrm{mg}, 0.0544 \mathrm{mmol})$ in $\mathrm{CH}_{2} \mathrm{Cl}_{2}(0.6 \mathrm{~mL})$ were added $\mathrm{NaHCO}_{3}(23 \mathrm{mg}, 0.273 \mathrm{mmol})$ and DMP $(34 \mathrm{mg}, 0.0806 \mathrm{mmol})$ at room temperature. The

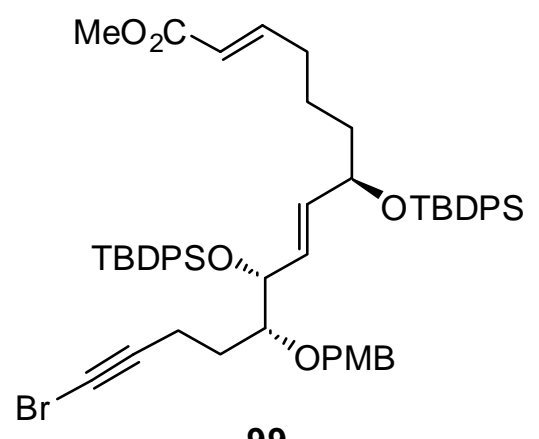

99 reaction mixture was stirred for 20 min before it was quenched with $\mathrm{NaHCO}_{3}\left(2 \mathrm{~mL}\right.$, sat. aq.) and $\mathrm{Na}_{2} \mathrm{~S}_{2} \mathrm{O}_{3}(2 \mathrm{~mL}$, sat. aq.). The resulting mixture was extracted with EtOAc $(3 \times 20 \mathrm{~mL})$, and the combined organic layers were dried $\left(\mathrm{Na}_{2} \mathrm{SO}_{4}\right)$ and concentrated in vacuo. To a solution of crude aldehyde $(43 \mathrm{mg}$, $0.047 \mathrm{mmol})$ in $\mathrm{CH}_{2} \mathrm{Cl}_{2}(1.0 \mathrm{~mL})$ was added $\mathrm{PPh}_{3}=\mathrm{CH}_{2} \mathrm{CO}_{2} \mathrm{Me}$

(19 $\mathrm{mg}, 0.056 \mathrm{mmol})$ at room temperature. The reaction mixture was stirred for $12 \mathrm{~h}$ before it was concentrated in vacuo. Flash column chromatography (silica gel, hexanes:EtOAc 10:1) afforded enoate 99 (40 mg, 88\%) as a colorless oil. 99: $R_{\mathrm{f}}=0.37$ (silica gel, hexanes:EtOAc 8:1); $[\alpha]_{\mathrm{D}}{ }^{25}=+33.4\left(\mathrm{CH}_{2} \mathrm{Cl}_{2, c}=2.0\right)$; IR (film) $v_{\max } 3048,2857,1725,1612,1513,1427,1246,1109$, 1037, $821 \mathrm{~cm}^{-1} ;{ }^{1} \mathrm{H}$ NMR (600 MHz, $\left.\mathrm{CDCl}_{3}\right): \delta=7.71-7.66(\mathrm{~m}, 8 \mathrm{H}), 7.49-7.31(\mathrm{~m}, 12 \mathrm{H}), 7.02$ $(\mathrm{d}, J=8.4 \mathrm{~Hz}, 2 \mathrm{H}), 6.89(\mathrm{dt}, J=15.6,7.8 \mathrm{~Hz}, 1 \mathrm{H}), 6.81(\mathrm{~d}, J=8.4 \mathrm{~Hz}, 2 \mathrm{H}), 5.76(\mathrm{~d}, J=15.6$ 
$\mathrm{Hz}, 1 \mathrm{H}), 5.73(\mathrm{dd}, J=15.6,6.0 \mathrm{~Hz}, 1 \mathrm{H}), 5.63(\mathrm{dd}, J=15.6,4.8 \mathrm{~Hz}, 1 \mathrm{H}), 4.45(\mathrm{t}, J=4.5 \mathrm{~Hz}, 1$ H), $4.24(\mathrm{q}, J=5.4 \mathrm{~Hz}, 1 \mathrm{H}), 4.10(\mathrm{~d}, J=11.4 \mathrm{~Hz}, 1 \mathrm{H}), 3.89(\mathrm{~d}, J=11.4 \mathrm{~Hz}, 1 \mathrm{H}), 3.82(\mathrm{~s}, 3 \mathrm{H})$, 3.78 (s, $3 \mathrm{H}), 3.31$ (ddd, $J=6.6,4.2,2.4 \mathrm{~Hz}, 1 \mathrm{H}), 2.28$ (ddd, $J=11.4,6.6,4.8 \mathrm{~Hz}, 1 \mathrm{H}), 2.19$ (ddd, $J=16.2,9.6,6.0 \mathrm{~Hz}, 1 \mathrm{H}), 2.02$ (pent, $J=5.8 \mathrm{~Hz}, 2 \mathrm{H}), 1.88-1.83(\mathrm{~m}, 1 \mathrm{H}), 1.43-1.28(\mathrm{~m}$, $5 \mathrm{H}), 1.15$ (s, $9 \mathrm{H}), 1.11 \mathrm{ppm}(\mathrm{s}, 9 \mathrm{H}) ;{ }^{13} \mathrm{C} \mathrm{NMR}\left(150 \mathrm{MHz}, \mathrm{CDCl}_{3}\right): \delta=167.1,159.0,149.4$, 136.2, 136.0, 136.0, 135.9, 135.4, 134.4, 134.1, 134.0, 133.9, 133.8, 130.8, 129.9, 129.6, 129.5, 129.2, 128.4, 127.7, 127.6, 127.5, 120.9, 113.6, 80.4, 80.0, 73.2, 72.1, 71.7, 55.2, 51.4, 37.7, 37.1, 32.1, 28.0, 27.1, 26.6, 22.7, 19.4, 16.2 ppm; HRMS (ESI): calcd for $\mathrm{C}_{56} \mathrm{H}_{67} \mathrm{BrO}_{6} \mathrm{Si}_{2} \mathrm{Na}^{+}\left[\mathrm{M}+\mathrm{Na}^{+}\right]$ 993.3552, found 993.3558 .

Vinyl stannane 100: To a mixture of the bromoacetylene 98 (153 mg, $0.167 \mathrm{mmol}), \mathrm{PPh}_{3}(8.8$ $\mathrm{mg}, 0.034 \mathrm{mmol})$ and $\mathrm{Pd}(\mathrm{dba})_{2}(9.6 \mathrm{mg}, 0.0167 \mathrm{mmol})$ in THF $(2 \mathrm{~mL})$ was added $n-\mathrm{Bu}_{3} \mathrm{SnH}$ mixture was stirred for $1 \mathrm{~h}$ before it was concentrated in
(0.09 $\mathrm{mL}, 0.334 \mathrm{mmol}$ ) at room temperature. The reaction
hexanes:EtOAc $12: 1)$ afforded vinyl stannane $\mathbf{1 0 0}$ (169 hexanes:EtOAc 9:1); $[\alpha]_{\mathrm{D}}{ }^{25}=+24.7\left(\mathrm{CH}_{2} \mathrm{Cl}_{2}, c=2.0\right)$; IR (film) $v_{\max } 3411,2929,2857,1612$, $1514,1428,1248,1111,1040,822 \mathrm{~cm}^{-1} ;{ }^{1} \mathrm{H}$ NMR $\left(600 \mathrm{MHz}, \mathrm{CDCl}_{3}\right): \delta=7.70-7.64(\mathrm{~m}, 8 \mathrm{H})$, 7.48-7.30 (m, $12 \mathrm{H}), 7.01(\mathrm{~d}, J=9.0 \mathrm{~Hz}, 2 \mathrm{H}), 6.77$ (d, $J=8.4 \mathrm{~Hz}, 2 \mathrm{H}), 5.97-5.80$ (m, $2 \mathrm{H})$, $5.64(\mathrm{t}, J=4.2 \mathrm{~Hz}, 2 \mathrm{H}), 4.40(\mathrm{t}, J=4.2 \mathrm{~Hz}, 1 \mathrm{H}), 4.18(\mathrm{q}, J=5.4 \mathrm{~Hz}, 1 \mathrm{H}), 4.06(\mathrm{~d}, J=11.4 \mathrm{~Hz}$, $1 \mathrm{H}), 3.93(\mathrm{~d}, J=10.8 \mathrm{~Hz}, 1 \mathrm{H}), 3.79(\mathrm{~s}, 3 \mathrm{H}), 3.42$ (q, $J=6.4 \mathrm{~Hz}, 2 \mathrm{H}), 3.13$ (ddd, $J=6.6,4.2$, $2.4 \mathrm{~Hz}, 1 \mathrm{H}), 2.70$ (oct, $J=4.8 \mathrm{~Hz}, 1 \mathrm{H}), 2.07-2.01(\mathrm{~m}, 1 \mathrm{H}), 1.81-1.75(\mathrm{~m}, 1 \mathrm{H}), 1.52$ (pent, $J=$ 
$7.8 \mathrm{~Hz}, 6 \mathrm{H}), 1.37-1.31$ (m, 7 H), 1.34 (hex, $J=7.2 \mathrm{~Hz}, 6 \mathrm{H}), 1.09$ (s, 9 H), 1.07 (s, 9 H), 0.92 (t, $J=7.5 \mathrm{~Hz}, 9 \mathrm{H}), 0.89 \mathrm{ppm}(\mathrm{t}, J=8.4 \mathrm{~Hz}, 6 \mathrm{H}) ;{ }^{13} \mathrm{C} \mathrm{NMR}\left(150 \mathrm{MHz}, \mathrm{CDCl}_{3}\right): \delta=158.9,149.5$, $136.0,136.0,136.0,135.9,134.7,134.2,134.1,134.0,133.9,131.1,129.8,129.6,129.5,129.4$, $129.0,128.5,127.6,127.5,127.5,127.4,127.3,113.5,81.6,73.5,72.7,71.5,62.8,55.2,37.2$, 34.6, 32.6, 29.2, 28.6, 27.3, 27.1, 20.4, 19.4, 19.3, 13.7, 9.4 ppm; HRMS (ESI): calcd for $\mathrm{C}_{65} \mathrm{H}_{94} \mathrm{O}_{5} \mathrm{Si}_{2} \mathrm{SnNa}^{+}\left[\mathrm{M}+\mathrm{Na}^{+}\right]$1153.5554, found 1153.5549.

Ester 102: To a solution of acid 101 (46 mg, $0.236 \mathrm{mmol})$ and DCC (49 mg, $0.236 \mathrm{mmol})$ in $\mathrm{CH}_{2} \mathrm{Cl}_{2}(0.5 \mathrm{~mL})$ was added 4-DMAP $(7.2 \mathrm{mg}, 0.059 \mathrm{mmol})$ at room temperature. The reaction

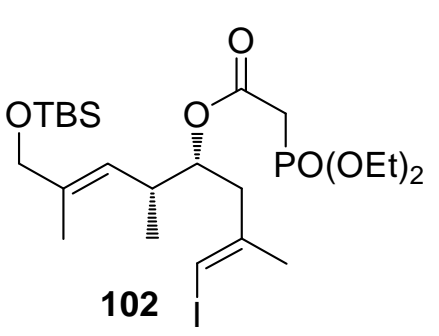
mixture was stirred for $15 \mathrm{~min}$ before a solution of alcohol $2(50 \mathrm{mg}$, $0.118 \mathrm{mmol})$ in $\mathrm{CH}_{2} \mathrm{Cl}_{2}(0.5 \mathrm{~mL})$ was added at room temperature. The reaction mixture was stirred for $12 \mathrm{~h}$ before it was quenched with $\mathrm{H}_{2} \mathrm{O}(10 \mathrm{~mL})$. The resulting mixture was extracted with EtOAc $(3 \times 20 \mathrm{~mL})$, and the combined organic layers were dried $\left(\mathrm{Na}_{2} \mathrm{SO}_{4}\right)$ and concentrated in vacuo. Flash column chromatography (silica gel, hexanes:EtOAc 2:1) afforded ester 102 (60 mg, 85\%) as a colorless oil. 102: $R_{\mathrm{f}}=0.40$ (silica gel, hexanes:EtOAc 2:1); $[\alpha]_{\mathrm{D}}{ }^{25}=+13.3\left(\mathrm{CH}_{2} \mathrm{Cl}_{2}, c=\right.$ 1.0); IR (film) $v_{\max } 2955,2930,2856,1736,1463,1391,1269,1053,838 \mathrm{~cm}^{-1} ;{ }^{1} \mathrm{H}$ NMR $(600$ $\left.\mathrm{MHz}, \mathrm{CDCl}_{3}\right): \delta=5.94(\mathrm{~s}, 1 \mathrm{H}), 5.21(\mathrm{~d}, J=10.2 \mathrm{~Hz}, 1 \mathrm{H}), 4.91(\mathrm{td}, J=8.4,3.0 \mathrm{~Hz}, 1 \mathrm{H}), 4.17$ (pent-d, $J=7.2,1.8 \mathrm{~Hz}, 4 \mathrm{H}), 4.00(\mathrm{~s}, 2 \mathrm{H}), 2.93(\mathrm{dd}, J=21.6,2.4 \mathrm{~Hz}, 2 \mathrm{H}), 2.63$ (dqd, $J=10.2$, 7.2, $3.0 \mathrm{~Hz}, 1 \mathrm{H}), 2.45$ (dd, $J=7.8,3.0 \mathrm{~Hz}, 1 \mathrm{H}), 2.38$ (dd, $J=14.4,9.0 \mathrm{~Hz}, 1 \mathrm{H}), 1.83(\mathrm{~s}, 3 \mathrm{H})$, 1.59 (s, $3 \mathrm{H}), 1.35$ (t, $J=7.2 \mathrm{~Hz}, 6 \mathrm{H}), 0.99$ (d, $J=7.2 \mathrm{~Hz}, 3 \mathrm{H}), 0.92$ (s, $9 \mathrm{H}), 0.07$ ppm (s, $6 \mathrm{H})$; ${ }^{13} \mathrm{C}$ NMR $\left(150 \mathrm{MHz}, \mathrm{CDCl}_{3}\right): \delta=165.3(\mathrm{~d}, J=4.5 \mathrm{~Hz}), 144.1,136.0,124.8,77.4,76.5,68.0$, 
$62.6(\mathrm{t}, J=6.0 \mathrm{~Hz}), 42.4,36.1,34.2(\mathrm{~d}, J=133.5 \mathrm{~Hz}), 25.9,23.9,18.4,16.8,16.4(\mathrm{~d}, J=6.0 \mathrm{~Hz})$, 13.9, -5.2 ppm; HRMS (ESI): calcd for $\mathrm{C}_{23} \mathrm{H}_{44} \mathrm{IO}_{6} \mathrm{PSiNa}^{+}\left[\mathrm{M}+\mathrm{Na}^{+}\right]$625.1582, found 625.1579.

Tetraene 103: To a mixture of vinyl stannane 100 (120 mg, $0.106 \mathrm{mmol})$, vinyl iodide 102 (50 $\mathrm{mg}, 0.083 \mathrm{mmol}), \mathrm{Pd}(\mathrm{dba})_{2}(15 \mathrm{mg}, 0.026 \mathrm{mmol}), \mathrm{AsPh}_{3}(55 \mathrm{mg}, 0.18 \mathrm{mmol})$ and $\mathrm{LiCl}(11 \mathrm{mg}$,

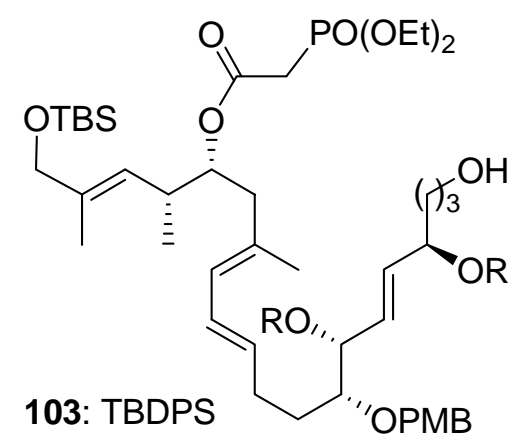
$0.27 \mathrm{mmol}$, freshly dried by heating with a heatgun for $20 \mathrm{~min}$ under vacuum) was added NMP $(1.0 \mathrm{~mL}$, degassed by using the freeze-thaw process sequence three times) at room temperature. The reaction mixture was stirred for $12 \mathrm{~h}$ before it was quenched with water $(15 \mathrm{~mL})$. The resulting mixture was extracted with EtOAc $(3 \times 30 \mathrm{~mL})$, and the combined organic layers were dried $\left(\mathrm{Na}_{2} \mathrm{SO}_{4}\right)$ and concentrated in vacuo. Flash column chromatography (silica gel, hexanes:EtOAc 2:1) afforded tetraene 103 (81 $\mathrm{mg}, 74 \%$ ) as a clear pale yellow oil. 103: $R_{\mathrm{f}}=0.30$ (silica gel, hexanes:EtOAc 2:1); $[\alpha]_{\mathrm{D}}^{25}=$ $+27.4\left(\mathrm{CH}_{2} \mathrm{Cl}_{2}, c=0.50\right)$; IR (film) $v_{\max } 2958,2930,2858,1733,1514,1463,1428,1275,1261$, 1112, 1055, 838, 764, $750 \mathrm{~cm}^{-1} ;{ }^{1} \mathrm{H}$ NMR $\left(600 \mathrm{MHz}, \mathrm{CDCl}_{3}\right): \delta=7.69-7.64(\mathrm{~m}, 8 \mathrm{H})$, 7.47-7.28 (m, $12 \mathrm{H}), 6.97$ (d, $J=8.4 \mathrm{~Hz}, 2 \mathrm{H}), 6.76(\mathrm{~d}, J=8.4 \mathrm{~Hz}, 2 \mathrm{H}), 6.12(\mathrm{dd}, J=15.0,10.8$ $\mathrm{Hz}, 1 \mathrm{H}), 5.74(\mathrm{~d}, J=11.4 \mathrm{~Hz}, 1 \mathrm{H}), 5.72(\mathrm{dd}, J=15.6,7.2 \mathrm{~Hz}, 1 \mathrm{H}), 5.56(\mathrm{dd}, J=15.6,4.8 \mathrm{~Hz}$, $1 \mathrm{H}), 5.48(\mathrm{dt}, J=15.0,7.2 \mathrm{~Hz}, 1 \mathrm{H}), 5.26(\mathrm{~d}, J=10.2 \mathrm{~Hz}, 1 \mathrm{H}), 4.96(\mathrm{td}, J=9.0,4.2 \mathrm{~Hz}, 1 \mathrm{H})$, $4.40(\mathrm{t}, J=4.2 \mathrm{~Hz}, 1 \mathrm{H}), 4.19-4.13(\mathrm{~m}, 1 \mathrm{H}), 4.16$ (hex, $J=7.2 \mathrm{~Hz}, 4 \mathrm{H}), 4.02(\mathrm{~s}, 2 \mathrm{H}), 4.01$ (d, $J$ $=11.4 \mathrm{~Hz}, 1 \mathrm{H}), 3.83(\mathrm{~d}, J=11.4 \mathrm{~Hz}, 1 \mathrm{H}), 3.78(\mathrm{~s}, 3 \mathrm{H}), 3.41(\mathrm{t}, J=6.6 \mathrm{~Hz}, 2 \mathrm{H}), 3.03(\mathrm{ddd}, J=$ 6.6, 4.2, $2.4 \mathrm{~Hz}, 1 \mathrm{H}$ ), 2.91 (ddt, $J=21.6,19.2,14.4 \mathrm{~Hz}, 2 \mathrm{H}$ ), 2.65 (ddq, $J=9.6,7.2,6.6 \mathrm{~Hz}, 1$ H), $2.33(\mathrm{dd}, J=13.8,3.6 \mathrm{~Hz}, 1 \mathrm{H}), 2.19(\mathrm{dd}, J=12.6,9.0 \mathrm{~Hz}, 1 \mathrm{H}), 2.17-2.11(\mathrm{~m}, 1 \mathrm{H}), 1.91$ (hex, $J=7.5 \mathrm{~Hz}, 1 \mathrm{H}), 1.77-1.59$ (m, $4 \mathrm{H}), 1.73$ (s, $3 \mathrm{H}), 1.61$ (s, $3 \mathrm{H}), 1.39-1.16$ (m, $4 \mathrm{H}), 1.34$ 
(qd, $J=7.2,4.2 \mathrm{~Hz}, 6 \mathrm{H}), 1.08$ (s, $9 \mathrm{H}), 1.06(\mathrm{~s}, 9 \mathrm{H}), 1.02(\mathrm{~d}, J=6.6 \mathrm{~Hz}, 3 \mathrm{H}), 0.92(\mathrm{~s}, 9 \mathrm{H})$, $0.08 \mathrm{ppm}(\mathrm{s}, 6 \mathrm{H}) ;{ }^{13} \mathrm{C} \mathrm{NMR}\left(150 \mathrm{MHz}, \mathrm{CDCl}_{3}\right): \delta=165.4,158.9,136.0,135.9,135.5,134.6$ 134.2, 134.2, 133.8, 133.6, 133.2, 131.7, 130.9, 129.8, 129.7, 129.5, 129.4, 129.1, 128.6, 127.9, $127.6,127.5,127.5,127.4,126.5,125.6,113.5,81.3,77.4,73.7,72.2,71.4,68.2,62.6(\mathrm{t}, J=$ $10.5 \mathrm{~Hz}), 62.6,55.2,42.9,37.4,36.0,34.2(\mathrm{~d}, J=135.0 \mathrm{~Hz}), 32.6,29.6,29.2,27.1,25.9,20.5$, 19.4, 19.3, 18.4, 16.6, 16.5, $16.3(\mathrm{~d}, J=6.0 \mathrm{~Hz}), 13.8,-5.2 \mathrm{ppm}$; HRMS (ESI): calcd for $\mathrm{C}_{76} \mathrm{H}_{111} \mathrm{O}_{11} \mathrm{PSi}_{3} \mathrm{Na}^{+}\left[\mathrm{M}+\mathrm{Na}^{+}\right]$1337.7064, found 1337.7020 .

Aldehyde 104: To a solution of alcohol $103(99 \mathrm{mg}, 0.075 \mathrm{mmol})$ in $\mathrm{CH}_{2} \mathrm{Cl}_{2}(1.0 \mathrm{~mL})$ were added $\mathrm{NaHCO}_{3}(35 \mathrm{mg}, 0.417 \mathrm{mmol})$ and DMP $(47 \mathrm{mg}, 0.111 \mathrm{mmol})$ at room temperature. The 104: $\mathrm{R}$ = TBDPS chromatography (silica gel, hexanes:EtOAc 2:1) afforded aldehyde $104(85 \mathrm{mg}, 85 \%)$ as a colorless oil. 104: $R_{\mathrm{f}}=0.34$ (silica gel, hexanes:EtOAc 3:1); $[\alpha]_{\mathrm{D}}{ }^{25}=+36.8\left(\mathrm{CH}_{2} \mathrm{Cl}_{2}, c=0.85\right.$ ); IR (film) $v_{\max } 2930,2857,1730,1612,1514,1463,1428,1390,1289,1270,1248,1110,1053$, $969,838,823,779 \mathrm{~cm}^{-1} ;{ }^{1} \mathrm{H}$ NMR $\left(600 \mathrm{MHz}, \mathrm{CDCl}_{3}\right): \delta=9.53(\mathrm{~s}, 1 \mathrm{H}), 7.68-7.62(\mathrm{~m}, 8 \mathrm{H})$, 7.46-7.29 (m, $12 \mathrm{H}), 6.98$ (d, $J=8.4 \mathrm{~Hz}, 2 \mathrm{H}), 6.77(\mathrm{~d}, J=8.4 \mathrm{~Hz}, 2 \mathrm{H}), 6.13(\mathrm{dd}, J=15.6,10.8$ $\mathrm{Hz}, 1 \mathrm{H}), 5.75(\mathrm{~d}, J=10.8 \mathrm{~Hz}, 1 \mathrm{H}), 5.68(\mathrm{dd}, J=15.6,6.0 \mathrm{~Hz}, 1 \mathrm{H}), 5.61(\mathrm{dd}, J=15.6,4.8 \mathrm{~Hz}$ $1 \mathrm{H}), 5.48$ (dt, $J=15.0,7.2 \mathrm{~Hz}, 1 \mathrm{H}), 5.27(\mathrm{~d}, J=9.6 \mathrm{~Hz}, 1 \mathrm{H}), 4.97$ (ddd, $J=11.4,7.8,4.2 \mathrm{~Hz}, 1$ H), $4.40(\mathrm{t}, J=4.2 \mathrm{~Hz}, 1 \mathrm{H}), 4.20-4.12(\mathrm{~m}, 1 \mathrm{H}), 4.16($ hex, $J=7.2 \mathrm{~Hz}, 4 \mathrm{H}), 4.03$ (d, $J=11.4$ 
$\mathrm{Hz}, 1 \mathrm{H}), 4.02$ (s, $2 \mathrm{H}), 3.86$ (d, $J=11.4 \mathrm{~Hz}, 1 \mathrm{H}), 3.78$ (s, $3 \mathrm{H}), 3.07$ (ddd, $J=6.6,4.2,2.4 \mathrm{~Hz}, 1$ H), 2.92 (ddt, $J=21.0,19.8,14.4 \mathrm{~Hz}, 2 \mathrm{H}), 2.66$ (ddq, $J=9.6,7.2,6.6 \mathrm{~Hz}, 1 \mathrm{H}), 2.34$ (dd, $J=$ 13.8, $3.6 \mathrm{~Hz}, 1 \mathrm{H}), 2.21(\mathrm{dd}, J=14.4,9.0 \mathrm{~Hz}, 1 \mathrm{H}), 2.17-2.11(\mathrm{~m}, 3 \mathrm{H}), 1.95$ (hex, $J=7.5 \mathrm{~Hz}, 1$ H), 1.77-1.69 (m, $4 \mathrm{H}), 1.73(\mathrm{~s}, 3 \mathrm{H}), 1.61(\mathrm{~s}, 3 \mathrm{H}), 1.54-1.45(\mathrm{~m}, 1 \mathrm{H}), 1.37-1.30(\mathrm{~m}, 1 \mathrm{H})$, $1.35(\mathrm{qd}, J=7.2,4.2 \mathrm{~Hz}, 6 \mathrm{H}), 1.08(\mathrm{~s}, 9 \mathrm{H}), 1.06(\mathrm{~s}, 9 \mathrm{H}), 1.02(\mathrm{~d}, J=6.6 \mathrm{~Hz}, 3 \mathrm{H}), 0.93(\mathrm{~s}, 9 \mathrm{H})$, $0.08 \mathrm{ppm}(\mathrm{s}, 6 \mathrm{H}) ;{ }^{13} \mathrm{C} \mathrm{NMR}\left(150 \mathrm{MHz}, \mathrm{CDCl}_{3}\right): \delta=202.5,165.4,158.9,136.0,135.9,135.4$ 134.4, 134.1, 134.0, 133.7, 133.5, 133.1, 131.7, 131.0, 129.8, 129.7, 129.6, 129.5, 129.1, 128.9, $127.9,127.7,127.5,127.5,126.7,125.6,113.5,81.2,77.3,73.1,72.3,71.4,68.2,62.6(\mathrm{~d}, J=6.0$ Hz), 55.2, 43.6, 42.8, 37.0, 35.9, 34.3 (d, $J=135.0 \mathrm{~Hz}), 29.6,29.2,27.0,26.0,19.4,19.3,18.4$, 17.1, 16.6, $16.4(\mathrm{~d}, J=6.0 \mathrm{~Hz}), 13.8,-5.2 \mathrm{ppm}$; $\mathrm{HRMS}$ (ESI): calcd for $\mathrm{C}_{76} \mathrm{H}_{109} \mathrm{O}_{11} \mathrm{PSi}_{3} \mathrm{Na}^{+}[\mathrm{M}+$ $\left.\mathrm{Na}^{+}\right]$1335.6908, found 1335.6872 .

Macrocycle 105: Method 1: To a solution of 18-crown-6 (120 mg, $0.454 \mathrm{mmol})$ in toluene (12 $\mathrm{mL})$ was added $\mathrm{K}_{2} \mathrm{CO}_{3}(25 \mathrm{mg}, 0.18 \mathrm{mmol}$, flame dried) at room temperature. The resulting

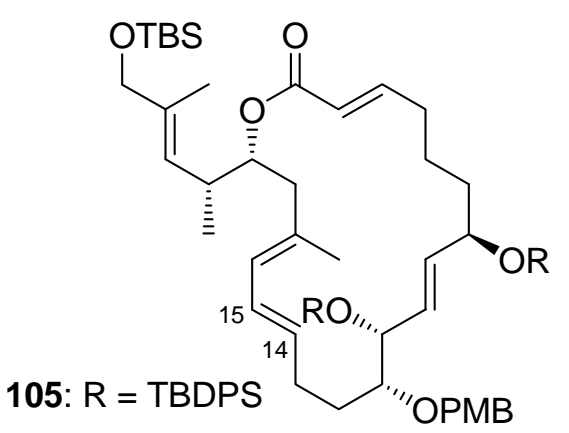
suspension was stirred for $3 \mathrm{~h}$ before a solution of phosphonate aldehyde $104(20 \mathrm{mg}, 0.015 \mathrm{mmol})$ in toluene (3 $\mathrm{mL}$ ) was added at room temperature. The mixture was stirred for $12 \mathrm{~h}$ before it was diluted with $\mathrm{Et}_{2} \mathrm{O}(50 \mathrm{~mL})$. The resulting mixture was washed with water $(2 \times 15 \mathrm{~mL})$, dried $\left(\mathrm{Na}_{2} \mathrm{SO}_{4}\right)$ and concentrated in vacuo. Flash column chromatography (silica gel, hexanes:EtOAc 10: 1) afforded macrocylce 105 (11.6 $\mathrm{mg}, 66 \%)$ as a colorless oil.

Method 2: To a solution of phosphonate aldehyde 104 (20 mg, $0.015 \mathrm{mmol})$ in $\mathrm{CH}_{3} \mathrm{CN}(15 \mathrm{~mL})$ were added $\mathrm{LiCl}\left(6.1 \mathrm{mg}, 0.15 \mathrm{mmol}\right.$, freshly flame-dried) and $i-\operatorname{Pr}_{2} \mathrm{NEt}(26 \mu \mathrm{L}, 0.15 \mathrm{mmol})$ at 
room temperature. The reaction mixture was stirred for $12 \mathrm{~h}$ before it was concentrated in vacuo. The resulting residue was dissolved in $\mathrm{Et}_{2} \mathrm{O}(50 \mathrm{~mL})$, washed with water $(2 \times 15 \mathrm{~mL})$, dried $\left(\mathrm{Na}_{2} \mathrm{SO}_{4}\right)$ and concentrated in vacuo. Flash column chromatography (silica gel, hexanes:EtOAc 10:1) afforded macrocylce $\mathbf{1 0 5}(12.9 \mathrm{mg}, 73 \%)$ as a colorless oil.

Method 3: To a suspension of $\mathrm{NaH}(60 \%$ in mineral oil, $1.6 \mathrm{mg}, 0.04 \mathrm{mmol})$ and molecular sieves $4 \AA(20 \mathrm{mg})$ in THF $(10 \mathrm{~mL})$ was added a solution of phosphonate aldehyde 104 (20 mg, $0.015 \mathrm{mmol})$ in THF $(5 \mathrm{~mL})$ at room temperature. The resulting suspension was stirred for $6 \mathrm{~h}$ before it was quenched with $\mathrm{H}_{2} \mathrm{O}(15 \mathrm{~mL})$. The resulting mixture was diluted with $\mathrm{Et}_{2} \mathrm{O}(50 \mathrm{~mL})$, organic layer was separated and washed with water $(2 \times 15 \mathrm{~mL})$, dried $\left(\mathrm{Na}_{2} \mathrm{SO}_{4}\right)$ and concentrated in vacuo. Flash column chromatography (silica gel, hexanes:EtOAc 10:1) afforded macrocylce $105(10.9 \mathrm{mg}, 62 \%)$ as a colorless oil.

Method 4: To a solution of the phosphonate aldehyde $104(20 \mathrm{mg}, 0.015 \mathrm{mmol})$ in THF (15 mL) was added NaHMDS (1.0 M in THF, $30 \mu \mathrm{L}, 0.030 \mathrm{mmol})$ at $-78{ }^{\circ} \mathrm{C}$. The reaction mixture was stirred for $10 \mathrm{~min}$ before it was warmed to $0{ }^{\circ} \mathrm{C}$ and stirred for further $2 \mathrm{~h}$. The reaction was quenched with $\mathrm{H}_{2} \mathrm{O}(15 \mathrm{~mL})$ and diluted with $\mathrm{Et}_{2} \mathrm{O}(50 \mathrm{~mL})$. The organic layer separated and washed with $\mathrm{H}_{2} \mathrm{O}(2 \times 15 \mathrm{~mL})$, dried $\left(\mathrm{Na}_{2} \mathrm{SO}_{4}\right)$ and concentrated in vacuo. Flash column chromatography (silica gel, hexanes:EtOAc 10:1) afforded macrocycle $105(8.8 \mathrm{mg}, 50 \%)$ as a colorless oil. 105: $R_{\mathrm{f}}=0.30$ (silica gel, hexanes:EtOAc 10:1); $[\alpha]_{\mathrm{D}}{ }^{25}=+18.0\left(\mathrm{CH}_{2} \mathrm{Cl}_{2, c}=0.30\right.$ ); IR (film) $v_{\max } 2931,2857,1720,1514,1472,1428,1361,1250,1112,837,702 \mathrm{~cm}^{-1} ;{ }^{1} \mathrm{H}$ NMR $\left(600 \mathrm{MHz}, \mathrm{CDCl}_{3}\right): \delta=7.74-7.64(\mathrm{~m}, 8 \mathrm{H}), 7.40-7.31(\mathrm{~m}, 12 \mathrm{H}), 6.95(\mathrm{~d}, J=8.4 \mathrm{~Hz}, 2 \mathrm{H}), 6.79$ (ddd, $J=14.4,8.4,5.4 \mathrm{~Hz}, 1 \mathrm{H}), 6.78(\mathrm{~d}, J=8.4 \mathrm{~Hz}, 2 \mathrm{H}), 6.06(\mathrm{dd}, J=15.0,11.4 \mathrm{~Hz}, 1 \mathrm{H}), 5.71$ (dd, $J=15.6,7.2 \mathrm{~Hz}, 1 \mathrm{H}), 5.68$ (d, $J=15.6 \mathrm{~Hz}, 1 \mathrm{H}), 5.59$ (d, $J=10.2 \mathrm{~Hz}, 1 \mathrm{H}), 5.40$ (dd, $J=$ 15.6, 4.2 Hz, $1 \mathrm{H}), 5.35$ (dt, $J=14.4,4.8 \mathrm{~Hz}, 1 \mathrm{H}), 5.26(\mathrm{~d}, J=10.2 \mathrm{~Hz}, 1 \mathrm{H}), 4.91$ (ddd, $J=10.2$, 
$8.4,1.8 \mathrm{~Hz}, 1 \mathrm{H}), 4.36$ (t, $J=3.3 \mathrm{~Hz}, 1 \mathrm{H}), 4.21(\mathrm{td}, J=6.6,3.6 \mathrm{~Hz}, 1 \mathrm{H}), 4.03(\mathrm{~s}, 2 \mathrm{H}), 4.02(\mathrm{~d}, J$ $=9.6 \mathrm{~Hz}, 1 \mathrm{H}), 3.79(\mathrm{~d}, J=11.4 \mathrm{~Hz}, 1 \mathrm{H}), 3.79(\mathrm{~s}, 3 \mathrm{H}), 2.99(\mathrm{ddd}, J=6.0,4.2,1.8 \mathrm{~Hz}, 1 \mathrm{H})$, 2.64 (hex, $J=7.5 \mathrm{~Hz}, 1 \mathrm{H}), 2.24(\mathrm{~d}, J=12.6 \mathrm{~Hz}, 1 \mathrm{H}), 2.04-1.89$ (m, $3 \mathrm{H}), 1.81-1.47$ (m, $4 \mathrm{H})$, $1.70(\mathrm{~s}, 3 \mathrm{H}), 1.62(\mathrm{~s}, 3 \mathrm{H}), 1.42-1.27$ (m, $4 \mathrm{H}), 1.14$ (s, $9 \mathrm{H}), 1.09$ (s, $9 \mathrm{H}), 0.97$ (d, $J=7.2 \mathrm{~Hz}, 3$ $\mathrm{H}), 0.93(\mathrm{~s}, 9 \mathrm{H}), 0.08 \mathrm{ppm}(\mathrm{s}, 6 \mathrm{H}) ;{ }^{13} \mathrm{C} \mathrm{NMR}\left(150 \mathrm{MHz}, \mathrm{CDCl}_{3}\right): \delta=166.5,159.0,148.5$, 136.0, 135.9, 135.5, 134.7, 134.4, 134.1, 133.6, 133.4, 133.2, 131.2, 130.8, 129.8, 129.7, 129.5, 129.4, 129.2, 129.1, 128.6, 127.6, 127.4, 127.4, 126.3, 125.7, 121.2, 113.6, 82.1, 74.8, 73.7, 72.4, $71.8,68.4,55.2,43.4,38.1,36.8,33.0,31.4,29.7,27.2,27.1,25.9,23.2,19.5,19.3,18.4,17.3$, 16.6, 13.9, -5.2 ppm; HRMS (ESI): calcd for $\mathrm{C}_{72} \mathrm{H}_{98} \mathrm{O}_{7} \mathrm{Si}_{3} \mathrm{Na}^{+}\left[\mathrm{M}+\mathrm{Na}^{+}\right] 1181.6513$, found 1181.6489 .

Vinyl stannane 106: To a mixture of the bromoacetylene 99 (720 mg, $0.75 \mathrm{mmol}), \mathrm{PPh}_{3}(39 \mathrm{mg}$, $0.15 \mathrm{mmol})$ and $\mathrm{Pd}(\mathrm{dba})_{2}(43 \mathrm{mg}, 0.075 \mathrm{mmol})$ in THF $(7.5 \mathrm{~mL})$ was added $n-\mathrm{Bu}_{3} \mathrm{SnH}(0.424$

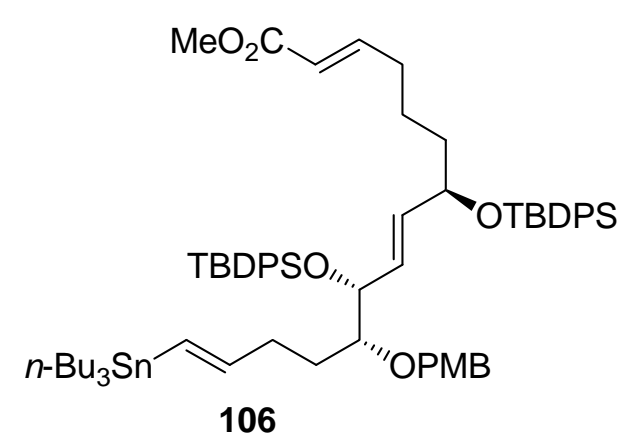
$\mathrm{mL}, 1.57 \mathrm{mmol}$ ) via syringe pump over a period of $30 \mathrm{~min}$ at room temperature. The reaction mixture was stirred for $1 \mathrm{~h}$ before it was concentrated in vacuo. Flash column chromatography (silica gel, hexanes:EtOAc 12:1) afforded vinyl stannane 106 (650 $\mathrm{mg}, 68 \%)$ as a colorless oil. 106: $R_{\mathrm{f}}=0.45$ (silica gel, hexanes:EtOAc 12:1); $[\alpha]_{\mathrm{D}}{ }^{25}=+15.3\left(\mathrm{CH}_{2} \mathrm{Cl}_{2}, c=1.9\right)$; IR (film) $v_{\max } 2929$, $2856,1726,1514,1463,1428,1248,1111,1041,741 \mathrm{~cm}^{-1} ;{ }^{1} \mathrm{H}$ NMR $\left(600 \mathrm{MHz}, \mathrm{CDCl}_{3}\right): \delta=$ 7.72-7.65 (m, $8 \mathrm{H}), 7.49-7.31(\mathrm{~m}, 12 \mathrm{H}), 7.04$ (d, $J=9.0 \mathrm{~Hz}, 2 \mathrm{H}), 6.89$ (dt, $J=15.0,7.8 \mathrm{~Hz}, 1$ H), $6.80(\mathrm{~d}, J=9.0 \mathrm{~Hz}, 2 \mathrm{H}), 6.01-5.89(\mathrm{~m}, 2 \mathrm{H}), 5.74(\mathrm{~d}, J=15.6 \mathrm{~Hz}, 1 \mathrm{H}), 5.65(\mathrm{t}, J=4.2 \mathrm{~Hz}$ $2 \mathrm{H}), 4.42(\mathrm{t}, J=3.9 \mathrm{~Hz}, 1 \mathrm{H}), 4.19(\mathrm{q}, J=4.8 \mathrm{~Hz}, 1 \mathrm{H}), 4.10(\mathrm{~d}, J=10.8 \mathrm{~Hz}, 1 \mathrm{H}), 3.97$ (d, $J=$ 
$10.8 \mathrm{~Hz}, 1 \mathrm{H}), 3.81$ (s, $3 \mathrm{H}), 3.77$ (s, $3 \mathrm{H}), 3.17$ (ddd, $J=6.6,4.2,2.4 \mathrm{~Hz}, 1 \mathrm{H}), 2.70$ (octet, $J=$ $4.8 \mathrm{~Hz}, 1 \mathrm{H}$ ), 2.08 (ddd, $J=15.0,9.0,6.0 \mathrm{~Hz}, 1 \mathrm{H}), 1.99$ (pent, $J=6.9 \mathrm{~Hz}, 2 \mathrm{H}), 1.82-1.76$ (m, 2 H), 1.54 (pent, $J=7.8 \mathrm{~Hz}, 6 \mathrm{H}), 1.45-1.24$ (m, $4 \mathrm{H}), 1.36$ (hex, $J=7.3 \mathrm{~Hz}, 6 \mathrm{H}), 1.11$ (s, $9 \mathrm{H})$, 1.08 (s, $9 \mathrm{H}), 0.94$ (t, $J=7.5 \mathrm{~Hz}, 9 \mathrm{H}), 0.91 \mathrm{ppm}(\mathrm{t}, J=7.5 \mathrm{~Hz}, 6 \mathrm{H}) ;{ }^{13} \mathrm{C}$ NMR $(150 \mathrm{MHz}$ $\left.\mathrm{CDCl}_{3}\right): \delta=167.1,158.9,149.5,149.4,136.0,136.0,135.9,134.5,134.1,134.0,133.9,131.1$, $129.8,129.6,129.6,129.4,129.0,128.7,127.6,127.5,127.4,127.3,120.9,113.5,81.5,73.3$, $72.8,71.5,55.2,51.3,37.0,34.6,32.1,29.2,28.7,27.3,27.1,22.7,19.4,19.3,13.8,9.4$ ppm; HRMS (ESI): calcd for $\mathrm{C}_{68} \mathrm{H}_{97} \mathrm{O}_{6} \mathrm{Si}_{2} \mathrm{Sn}^{+}\left[\mathrm{M}+\mathrm{H}^{+}\right]$1185.5840, found 1185.5832.

Carboxylic acid 107: To a solution of ester $106(120 \mathrm{mg}, 0.101 \mathrm{mmol})$ in 1,2-dichloroethane $(2.0 \mathrm{~mL})$ was added $\mathrm{Me}_{3} \mathrm{SnOH}(190 \mathrm{mg}, 1.01 \mathrm{mmol})$ at room temperature. The reaction mixture was heated at $90{ }^{\circ} \mathrm{C}$ for $12 \mathrm{~h}$ before it was concentrated in
vacuo. The resulting residue was dissolved with EtOAc
$(60 \mathrm{~mL})$, washed with $\mathrm{HCl}(3.0 \mathrm{~N}$ aq., $10 \mathrm{~mL})$, dried
$\left(\mathrm{Na}_{2} \mathrm{SO}_{4}\right)$ and concentrated in vacuo. Flash column
chromatography (silica gel, hexanes:EtOAc 3:1) afforded carboxylic acid 107 (83 mg, 70\%) as a colorless oil.

Ester 108: To a solution of carboxylic acid $107(70 \mathrm{mg}, 0.06 \mathrm{mmol})$ and $\mathrm{Et}_{3} \mathrm{~N}(0.042 \mathrm{~mL}, 0.30$ mmol) in toluene $(1.0 \mathrm{~mL})$ was added 2,4,6-trichlorobenzoyl chloride $(14 \mu \mathrm{L}, 0.09 \mathrm{mmol})$ at room temperature. The reaction mixture was stirred for $1 \mathrm{~h}$ before a solution of alcohol $2(31 \mathrm{mg}$, $0.073 \mathrm{mmol})$ and 4-DMAP $(7.3 \mathrm{mg}, 0.06 \mathrm{mmol})$ in toluene $(1.0 \mathrm{~mL})$ was added at room temperature. The reaction mixture was stirred at for $3 \mathrm{~h}$ before it was quenched with $\mathrm{NH}_{4} \mathrm{Cl}(10$ 


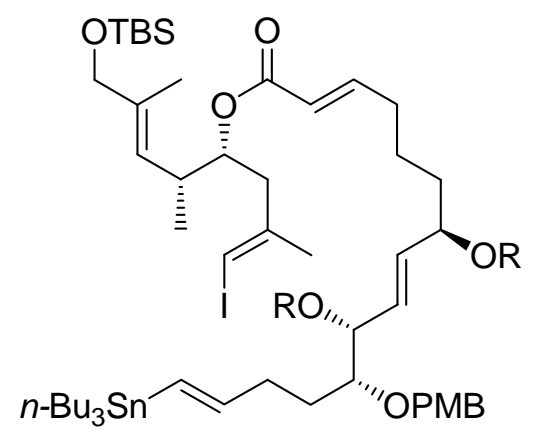

108: $R=$ TBDPS

$\mathrm{mL}$, sat. aq.). The resulting mixture was extracted with $\mathrm{Et}_{2} \mathrm{O}(3$ $\times 20 \mathrm{~mL})$, and the combined organic layers were dried $\left(\mathrm{Na}_{2} \mathrm{SO}_{4}\right)$ and concentrated in vacuo. Flash column chromatography (silica gel, hexanes:EtOAc 12:1) afforded ester 108 (74 mg, 78\%) as a colorless oil. 108: $R_{\mathrm{f}}=0.40$ (silica gel, hexanes:EtOAc 14:1); $[\alpha]_{\mathrm{D}}^{25}=+20.9\left(c=\mathrm{CH}_{2} \mathrm{Cl}_{2}, 0.42\right)$; IR (film) $v_{\max } 2929,2856,1720,1653,1514,1463,1428,1249,1111,1073,837,822,702 \mathrm{~cm}^{-1} ;{ }^{1} \mathrm{H}$ $\operatorname{NMR}\left(600 \mathrm{MHz}, \mathrm{CDCl}_{3}\right): \delta=7.68-7.62(\mathrm{~m}, 8 \mathrm{H}), 7.46-7.29(\mathrm{~m}, 12 \mathrm{H}), 7.01(\mathrm{~d}, J=8.4 \mathrm{~Hz}, 2 \mathrm{H})$, $6.81(\mathrm{dt}, J=15.6,7.4 \mathrm{~Hz}, 1 \mathrm{H}), 6.77$ (d, $J=8.4 \mathrm{~Hz}, 2 \mathrm{H}), 5.96-5.82(\mathrm{~m}, 2 \mathrm{H}), 5.89$ (s, $1 \mathrm{H}), 5.68$ $(\mathrm{d}, J=15.6 \mathrm{~Hz}, 1 \mathrm{H}), 5.62(\mathrm{t}, J=5.4 \mathrm{~Hz}, 2 \mathrm{H}), 5.26(\mathrm{~d}, J=10.2 \mathrm{~Hz}, 1 \mathrm{H}), 4.95(\mathrm{td}, J=8.4,3.0$ $\mathrm{Hz}, 1 \mathrm{H}), 4.38(\mathrm{t}, J=4.2 \mathrm{~Hz}, 1 \mathrm{H}), 4.16(\mathrm{q}, J=5.1 \mathrm{~Hz}, 1 \mathrm{H}), 4.07(\mathrm{~d}, J=11.4 \mathrm{~Hz}, 1 \mathrm{H}), 4.02(\mathrm{~s}, 2$ H), $3.94(\mathrm{~d}, J=10.8 \mathrm{~Hz}, 1 \mathrm{H}), 3.79$ (s, $3 \mathrm{H}), 3.13$ (ddd, $J=6.6,3.6,3.0 \mathrm{~Hz}, 1 \mathrm{H}), 2.65$ (ddq, $J=$ 9.6, 7.2, $6.6 \mathrm{~Hz}, 1 \mathrm{H}), 2.48(\mathrm{dd}, J=14.4,3.0 \mathrm{~Hz}, 1 \mathrm{H}), 2.40(\mathrm{dd}, J=13.8,9.0 \mathrm{~Hz}, 1 \mathrm{H})$, 2.29-2.23 (m, $2 \mathrm{H}), 2.07-2.01(\mathrm{~m}, 1 \mathrm{H}), 1.95$ (pent, $J=6.9 \mathrm{~Hz}, 2 \mathrm{H}), 1.85(\mathrm{~s}, 3 \mathrm{H}), 1.78-1.72(\mathrm{~m}$, $1 \mathrm{H}), 1.61(\mathrm{~s}, 3 \mathrm{H}), 1.51$ (pent, $J=7.8 \mathrm{~Hz}, 6 \mathrm{H}), 1.41-1.29(\mathrm{~m}, 5 \mathrm{H}), 1.33$ (hex, $J=7.2 \mathrm{~Hz}, 6 \mathrm{H})$, $1.08(\mathrm{~s}, 9 \mathrm{H}), 1.05(\mathrm{~s}, 9 \mathrm{H}), 0.97(\mathrm{~d}, J=6.6 \mathrm{~Hz}, 3 \mathrm{H}), 0.93(\mathrm{~s}, 9 \mathrm{H}), 0.91(\mathrm{t}, J=7.5 \mathrm{~Hz}, 9 \mathrm{H}), 0.88$ (t, $J=8.4 \mathrm{~Hz}, 6 \mathrm{H}), 0.08 \mathrm{ppm}(\mathrm{s}, 6 \mathrm{H}) ;{ }^{13} \mathrm{C} \mathrm{NMR}\left(150 \mathrm{MHz}, \mathrm{CDCl}_{3}\right): \delta=166.1,158.9,149.5$, $149.4,144.3,136.0,136.0,135.9,135.8,134.5,134.1,134.0,133.9,133.8,131.1,129.8,129.6$, $129.6,129.4,129.0,128.7,127.6,127.5,127.5,127.4,127.3,125.2,120.9,113.5,81.4,74.8$, $73.3,72.8,71.5,68.1,55.2,42.3,37.2,36.2,34.6,32.1,29.2,28.6,27.3,27.1,25.9,22.8,22.6$, 19.4, 18.4, 16.9, 13.9, 13.7, 9.4, -5.2 ppm; HRMS (ESI): calcd for $\mathrm{C}_{84} \mathrm{H}_{126} \mathrm{IO}_{7} \mathrm{Si}_{3} \mathrm{Sn}^{+}\left[\mathrm{M}+\mathrm{H}^{+}\right]$ 1599.6692, found 1599.6701. 
Macrocycle 105: To a solution of vinyl stannane vinyl iodide 108 (16 mg, $0.01 \mathrm{mmol})$ in NMP (10 $\mathrm{mL}$, degassed by using the freeze-thaw process sequence three times) were added $\operatorname{Pd}(\mathrm{dba})_{2}$

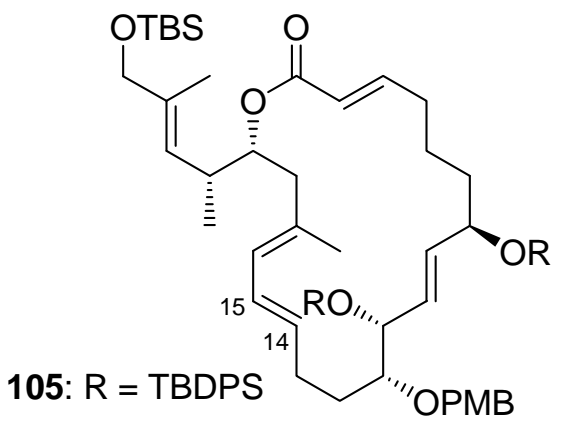
(2.3 mg, $0.004 \mathrm{mmol}), \mathrm{AsPh}_{3}(6.0 \mathrm{mg}, 0.02 \mathrm{mmol})$ and $\mathrm{LiCl}$ (1.2 $\mathrm{mg}, 0.03 \mathrm{mmol}$, freshly dried by heating with a heatgun for 20 min under vacuum) at room temperature. The reaction mixture was stirred for $12 \mathrm{~h}$ before it was quenched with water $(10 \mathrm{~mL})$. The resulting mixture was extracted with EtOAc $(3 \times 20 \mathrm{~mL})$, and the combined organic layers were dried $\left(\mathrm{Na}_{2} \mathrm{SO}_{4}\right)$ and concentrated in vacuo. Flash column chromatography (silica gel, hexanes:EtOAc 10:1) afforded macrocycle 105 [7.4 mg, 67\%; this material was contaminated with an isomer of $\mathbf{1 0 5}$, in ca. 45:22 ratio in favor of $\mathbf{1 0 5}$ (determined by ${ }^{1} \mathrm{H}$ NMR spectroscopic analysis), which could not be chromatographically removed].

Pentaene 109: To a mixture of vinyl stannane 106 (145 mg, $0.123 \mathrm{mmol}$ ), vinyl iodide 2 (52 mg, $0.123 \mathrm{mmol}), \mathrm{Pd}(\mathrm{dba})_{2}(21 \mathrm{mg}, 0.037 \mathrm{mmol}), \mathrm{AsPh}_{3}(77 \mathrm{mg}, 0.246 \mathrm{mmol})$ and $\mathrm{LiCl}(15 \mathrm{mg}, 0.37$

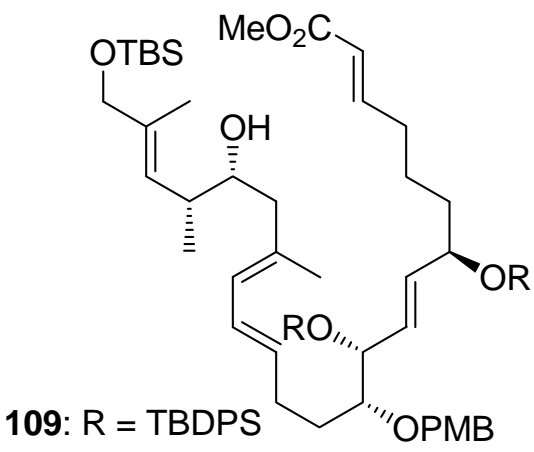
mmol, freshly dried by heating with a heatgun for $20 \mathrm{~min}$ under vacuum) was added NMP (1.5 mL, degassed by using the freeze-thaw process sequence three times) at room temperature. The reaction mixture was stirred for $12 \mathrm{~h}$ before it was quenched with water $(15 \mathrm{~mL})$. The resulting mixture was extracted with EtOAc $(3 \times 30 \mathrm{~mL})$, and the combined organic layers were dried $\left(\mathrm{Na}_{2} \mathrm{SO}_{4}\right)$ and concentrated in vacuo. Flash column chromatography (silica gel, hexanes:EtOAc 4:1) afforded pentaene $109(107 \mathrm{mg}, 73 \%)$ as a clear pale yellow oil. 109: $R_{\mathrm{f}}=0.30$ (silica gel, 
hexanes:EtOAc 4:1); $[\alpha]_{\mathrm{D}}{ }^{25}=+36.7\left(\mathrm{CH}_{2} \mathrm{Cl}_{2,} c=0.52\right)$; IR (film) $v_{\max } 3450,2931,2858,1726$, $1612,1514,1463,1428,1250,1111,838,702 \mathrm{~cm}^{-1} ;{ }^{1} \mathrm{H}$ NMR $\left(600 \mathrm{MHz}, \mathrm{CDCl}_{3}\right): \delta=7.69-7.64$ (m, $8 \mathrm{H}), 7.47-7.28(\mathrm{~m}, 12 \mathrm{H}), 6.98(\mathrm{~d}, J=9.0 \mathrm{~Hz}, 2 \mathrm{H}), 6.83(\mathrm{dt}, J=15.6,6.9 \mathrm{~Hz}, 1 \mathrm{H}), 6.77(\mathrm{~d}$, $J=8.4 \mathrm{~Hz}, 2 \mathrm{H}), 6.16(\mathrm{dd}, J=15.0,10.8 \mathrm{~Hz}, 1 \mathrm{H}), 5.84(\mathrm{~d}, J=10.2 \mathrm{~Hz}, 1 \mathrm{H}), 5.71(\mathrm{~d}, J=15.6$ $\mathrm{Hz}, 1 \mathrm{H}), 5.69$ (dd, $J=15.6,6.0 \mathrm{~Hz}, 1 \mathrm{H}), 5.58$ (dd, $J=15.6,4.8 \mathrm{~Hz}, 1 \mathrm{H}), 5.50$ (dt, $J=15.0,7.3$ $\mathrm{Hz}, 1 \mathrm{H}), 5.30(\mathrm{~d}, J=10.2 \mathrm{~Hz}, 1 \mathrm{H}), 4.40(\mathrm{t}, J=3.9 \mathrm{~Hz}, 1 \mathrm{H}), 4.18(\mathrm{q}, J=5.6 \mathrm{~Hz}, 1 \mathrm{H})$, 4.08-4.00 (m, $1 \mathrm{H}), 4.05$ (s, $2 \mathrm{H}), 4.04(\mathrm{~d}, J=12.0 \mathrm{~Hz}, 1 \mathrm{H}), 3.84$ (d, $J=11.4 \mathrm{~Hz}, 1 \mathrm{H}), 3.78(\mathrm{~s}$, $3 \mathrm{H}), 3.74$ (s, $3 \mathrm{H}), 3.53$ (ddd, $J=10.2,7.2,3.0 \mathrm{~Hz}, 1 \mathrm{H}), 3.06$ (ddd, $J=6.6,4.2,2.4 \mathrm{~Hz}, 1 \mathrm{H})$, $2.49(\mathrm{ddq}, J=9.6,6.6,6.6 \mathrm{~Hz}, 1 \mathrm{H}), 2.35(\mathrm{~d}, J=13.2 \mathrm{~Hz}, 1 \mathrm{H}), 2.19-2.14(\mathrm{~m}, 1 \mathrm{H}), 2.00-1.94$ (m, 4 H), 1.79-1.60 (m, 2 H), 1.77 (s, 3 H), 1.64 (s, 3 H), 1.38-1.31 (m, 4 H), 1.08 (s, 9 H), 1.07 $(\mathrm{d}, J=6.6 \mathrm{~Hz}, 3 \mathrm{H}), 1.06$ (s, $9 \mathrm{H}), 0.94(\mathrm{~s}, 9 \mathrm{H}), 0.09 \mathrm{ppm}(\mathrm{s}, 6 \mathrm{H}) ;{ }^{13} \mathrm{C} \mathrm{NMR}\left(150 \mathrm{MHz}, \mathrm{CDCl}_{3}\right)$ : $\delta=167.1,158.9,149.4,136.0,135.9,135.9,135.0,134.5,134.2,134.0,133.7,133.6,133.5$ $132.8,131.0,129.8,129.7,129.6,129.4,129.0,128.7,128.0,127.7,127.53,127.50,127.4,126.7$, $126.5,120.8,113.5,81.0,73.4,73.0,72.3,71.4,68.4,55.2,51.3,45.7,38.3,37.1,32.1,29.5$, 29.2, 27.1, 26.0, 22.8, 19.4, 19.3, 18.4, 16.7, 16.5, 13.9, -5.2 ppm; HRMS (ESI): calcd for $\mathrm{C}_{73} \mathrm{H}_{102} \mathrm{O}_{8} \mathrm{Si}_{3} \mathrm{Na}^{+}\left[\mathrm{M}+\mathrm{Na}^{+}\right]$1213.6775, found 1213.6779.

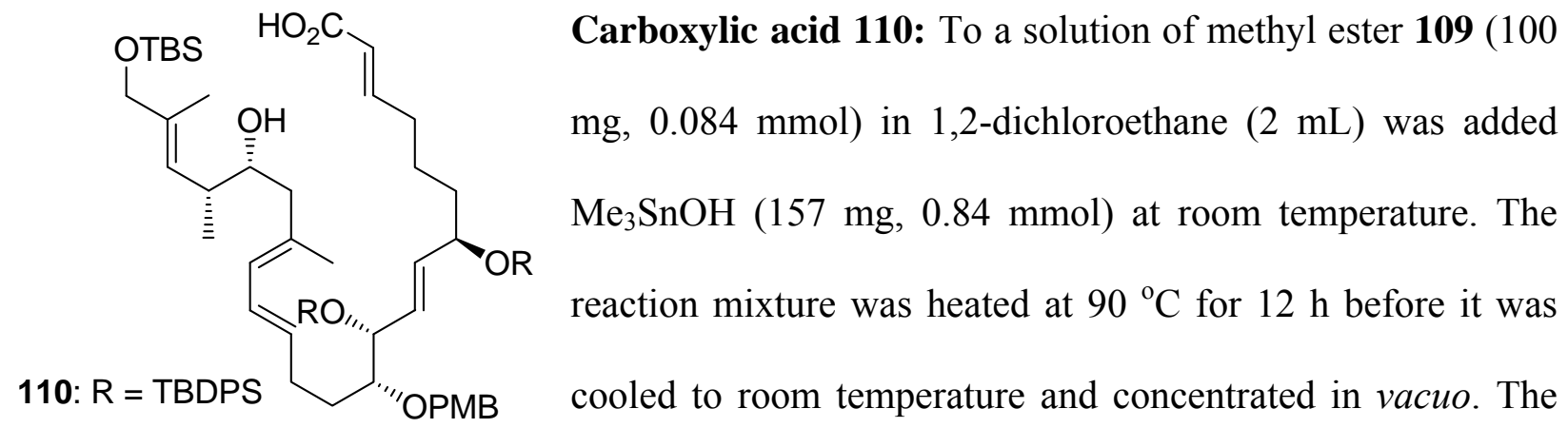
resulting residue was dissolved in EtOAc $(60 \mathrm{~mL})$, washed with $\mathrm{HCl}(3.0 \mathrm{~N}$ aq., $10 \mathrm{~mL})$, dried 
$\left(\mathrm{Na}_{2} \mathrm{SO}_{4}\right)$ and concentrated in vacuo. Flash column chromatography (silica gel, hexanes:EtOAc 3:1) afforded carboxylic acid $\mathbf{1 1 0}(85 \mathrm{mg}, 86 \%)$ as a colorless oil.

Macrocycle 105: To a solution of seco acid $110(20 \mathrm{mg}, 0.017 \mathrm{mmol})$ in THF $(2.0 \mathrm{~mL})$ were added $\mathrm{Et}_{3} \mathrm{~N}(47 \mu \mathrm{L}, 0.34 \mathrm{mmol})$ and 2,4,6-trichlorobenzoyl chloride $(40 \mu \mathrm{L}, 0.255 \mathrm{mmol})$ at

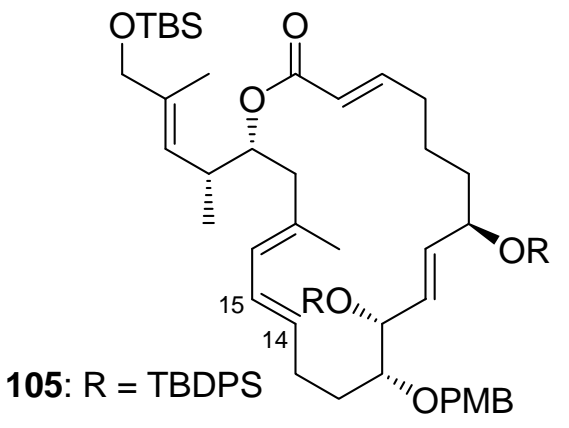
room temperature. The reaction mixture was stirred for $2 \mathrm{~h}$ before a solution of 4-DMAP ( $83 \mathrm{mg}, 0.68 \mathrm{rnmol})$ in toluene $(17 \mathrm{~mL})$ was added via syringe pump over a period of $2 \mathrm{~h}$ at room temperature. The reaction mixture was stirred for $12 \mathrm{~h}$ before it was quenched with $\mathrm{NH}_{4} \mathrm{Cl}(20 \mathrm{~mL}$, sat. aq.). The resulting mixture was extracted with EtOAc $(3 \times 20 \mathrm{~mL})$, and the combined organic layers were dried $\left(\mathrm{Na}_{2} \mathrm{SO}_{4}\right)$ and concentrated in vacuo. Flash column chromatography (silica gel, hexanes:EtOAc 10:1) afforded macrocycle 105 (16.0 mg, 81\%) as a colorless oil. All physical properties of this compound were identical to those reported for macrocycle 105 obtained from phosphonate aldehyde 104.

Pentaene 111: To a mixture of vinyl stannane 106 (140 mg, 0.119 mmol), vinyl iodide 19-epi-2 (50 mg, $0.119 \mathrm{mmol}), \mathrm{Pd}(\mathrm{dba})_{2}(20 \mathrm{mg}, 0.036 \mathrm{mmol}), \mathrm{AsPh}_{3}(74 \mathrm{mg}, 0.238 \mathrm{mmol})$ and $\mathrm{LiCl}$ (14.5 mg, $0.36 \mathrm{mmol}$, freshly dried by heating with a heatgun for 20 min under vacuum) was added NMP (1.4 mL, degassed by using the freeze-thaw process sequence three times) at room temperature. The resulting suspension was stirred for $12 \mathrm{~h}$ before it was quenched with water (15 $\mathrm{mL})$. The resulting mixture was extracted with EtOAc $(3 \times 30 \mathrm{~mL})$, and the combined organic layers were dried $\left(\mathrm{Na}_{2} \mathrm{SO}_{4}\right)$ and concentrated in vacuo. Flash column chromatography (silica gel, 


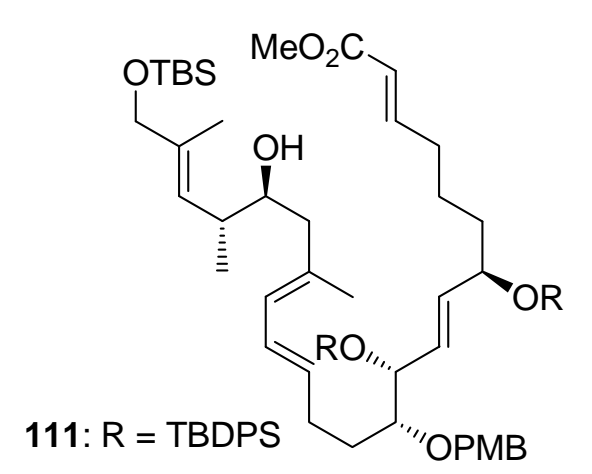

hexanes:EtOAc 4:1) afforded pentaene $\mathbf{1 1 1}(100 \mathrm{mg}, 71 \%)$ as a clear yellow oil. 111: $R_{\mathrm{f}}=0.30$ (silica gel, hexanes:EtOAc $4: 1) ;[\alpha]_{D}^{25}=+30.2\left(\mathrm{CH}_{2} \mathrm{Cl}_{2}, c=0.58\right) ;$ IR (film) $v_{\max } 3500$, $2930,2857,1726,1612,1513,1462,1428,1247,1111,837$, $702 \mathrm{~cm}^{-1} ;{ }^{1} \mathrm{H}$ NMR $\left(600 \mathrm{MHz}, \mathrm{CDCl}_{3}\right): \delta=7.69-7.63(\mathrm{~m}, 8$ H), 7.47-7.29 (m, $12 \mathrm{H}), 6.99$ (d, $J=9.0 \mathrm{~Hz}, 2 \mathrm{H}), 6.83$ (dt, $J=15.6,6.9 \mathrm{~Hz}, 1 \mathrm{H}), 6.77$ (d, $J=$ $8.4 \mathrm{~Hz}, 2 \mathrm{H}), 6.16(\mathrm{dd}, J=15.0,10.8 \mathrm{~Hz}, 1 \mathrm{H}), 5.84(\mathrm{~d}, J=10.2 \mathrm{~Hz}, 1 \mathrm{H}), 5.71(\mathrm{~d}, J=15.6 \mathrm{~Hz}, 1$ H), $5.68(\mathrm{dd}, J=15.6,6.6 \mathrm{~Hz}, 1 \mathrm{H}), 5.59(\mathrm{dd}, J=15.6,5.4 \mathrm{~Hz}, 1 \mathrm{H}), 5.50(\mathrm{dt}, J=15.0,7.2 \mathrm{~Hz}, 1$ H), $5.37(\mathrm{~d}, J=10.8 \mathrm{~Hz}, 1 \mathrm{H}), 4.39(\mathrm{t}, J=4.2 \mathrm{~Hz}, 1 \mathrm{H}), 4.18(\mathrm{q}, J=5.4 \mathrm{~Hz}, 1 \mathrm{H}), 4.07-4.03(\mathrm{~m}$, $1 \mathrm{H}), 4.07$ (s, $2 \mathrm{H}), 4.03(\mathrm{~d}, J=11.4 \mathrm{~Hz}, 1 \mathrm{H}), 3.84(\mathrm{~d}, J=11.4 \mathrm{~Hz}, 1 \mathrm{H}), 3.78(\mathrm{~s}, 3 \mathrm{H}), 3.74(\mathrm{~s}, 3$ H), 3.60 (ddd, $J=8.4,5.4,3.0 \mathrm{~Hz}, 1 \mathrm{H}), 3.07$ (ddd, $J=6.6,4.8,2.4 \mathrm{~Hz}, 1 \mathrm{H}), 2.54$ (dqd, $J=9.6$, 7.2, $6.6 \mathrm{~Hz}, 1 \mathrm{H}), 2.28$ (d, $J=13.2 \mathrm{~Hz}, 1 \mathrm{H}), 2.22-2.14(\mathrm{~m}, 1 \mathrm{H}), 2.09$ (dd, $J=13.2,9.6 \mathrm{~Hz}, 1 \mathrm{H})$, 2.02-1.94 (m, 3 H), 1.82-1.60 (m, 2 H), 1.78 (s, 3 H), 1.66 (s, 3 H), 1.36-1.24 (m, 4 H), 1.08 (s, $9 \mathrm{H}), 1.06$ (s, $9 \mathrm{H}), 1.04$ (d, $J=6.6 \mathrm{~Hz}, 3 \mathrm{H}), 0.94$ (s, $9 \mathrm{H}), 0.09 \mathrm{ppm}(\mathrm{s}, 6 \mathrm{H}) ;{ }^{13} \mathrm{C}$ NMR $(150$ $\left.\mathrm{MHz}, \mathrm{CDCl}_{3}\right): \delta=167.1,158.9,149.4,136.0,136.0,135.9,135.9,134.6,134.5,134.1,134.0$ 133.7, 133.6, 133.4, 132.8, 131.0, 129.8, 129.65, 129.56, 129.4, 129.0, 128.7, 127.9, 127.6, 127.5, $127.5,127.4,126.6,126.0,120.8,113.5,80.9,73.4,72.9,72.3,71.4,68.6,55.2,51.3,44.8,37.9$, 37.1, 32.1, 29.4, 29.1, 27.1, 26.0, 22.8, 19.4, 19.3, 18.4, 16.9, 16.7, 13.9, -5.2 ppm; HRMS (ESI): calcd for $\mathrm{C}_{73} \mathrm{H}_{102} \mathrm{O}_{8} \mathrm{Si}_{3} \mathrm{Na}^{+}\left[\mathrm{M}+\mathrm{Na}^{+}\right]$1213.6775, found 1213.6784.

Carboxylic acid 112: To a solution of methyl ester 111 (56 mg, $0.047 \mathrm{mmol}$ ) in 1,2dichloroethane $(1.0 \mathrm{~mL})$ was added $\mathrm{Me}_{3} \mathrm{SnOH}(88 \mathrm{mg}, 0.47 \mathrm{mmol})$ at room temperature. The reaction mixture was heated at $90{ }^{\circ} \mathrm{C}$ for $12 \mathrm{~h}$ before it was cooled to room temperature and 


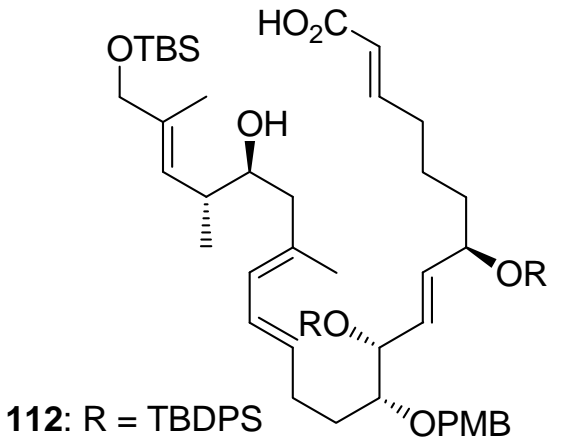

concentrated in vacuo. The resulting residue was dissolved in EtOAc $(60 \mathrm{~mL})$, washed with $\mathrm{HCl}(3.0 \mathrm{~N}$ aq., $10 \mathrm{~mL})$, dried $\left(\mathrm{Na}_{2} \mathrm{SO}_{4}\right)$ and concentrated in vacuo. Flash column chromatography (silica gel, hexanes:EtOAc 3:1) afforded carboxylic acid $112(44 \mathrm{mg}, 80 \%)$ as a colorless oil.

Macrocycle 105: Method 1: To a solution of $\mathrm{Ph}_{3} \mathrm{P}(18.0 \mathrm{mg}, 0.068 \mathrm{mmol})$ in THF $(5.0 \mathrm{~mL})$ was added DIAD (13 $\mu \mathrm{L}, 0.068 \mathrm{mmol})$ at room temperature. The reaction mixture was stirred for 30

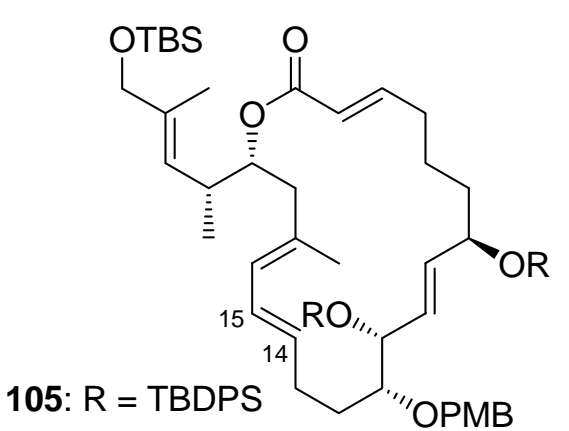
min before a solution of carboxylic acid $\mathbf{1 1 2}(8 \mathrm{mg}, 0.0068$ mmol) in THF (1.8 $\mathrm{mL})$ was added via syringe pump over a period of $1 \mathrm{~h}$ at room temperature. Only starting material 112 was recovered.

Method 2: To a solution of $\mathrm{Ph}_{3} \mathrm{P}(18.0 \mathrm{mg}, 0.068 \mathrm{mmol})$ in benzene $(5.0 \mathrm{~mL})$ was added DIAD $(13 \mu \mathrm{L}, 0.068 \mathrm{mmol})$ at room temperature. The reaction mixture was stirred for $30 \mathrm{~min}$ before a solution of carboxylic acid $112(8 \mathrm{mg}, 0.0068 \mathrm{mmol})$ in benzene $(1.8 \mathrm{~mL})$ was added via syringe pump over a period of $1 \mathrm{~h}$ at room temperature. Only starting material 112 was recovered.

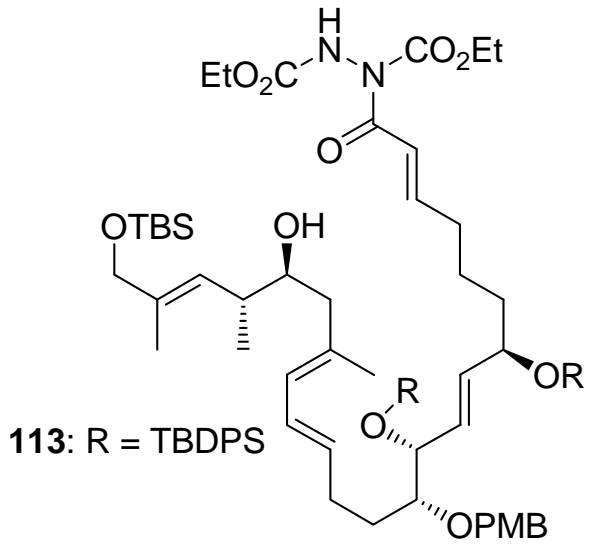

Method 3: To a solution of $\mathrm{Ph}_{3} \mathrm{P}(26.0 \mathrm{mg}, 0.1 \mathrm{mmol})$ in THF $(8.0 \mathrm{~mL})$ was added DEAD $(16 \mu \mathrm{L}, 0.1 \mathrm{mmol})$ at room temperature. The reaction mixture was stirred for 30 min before a solution of acid $112(12 \mathrm{mg}, 0.01 \mathrm{mmol})$ in THF $(2.0 \mathrm{~mL})$ was added via syringe pump over a period of $1 \mathrm{~h}$ at room temperature and then concentrated in vacuo. 
Flash column chromatography (silica gel, hexanes:EtOAc 4:1) afforded compound 113 (5.6 mg, 40\%) as a colorless oil. The desired macrocycle $\mathbf{1 0 5}$ was not observed in this reaction. Structure 113 was tentatively assigned on the basis of LCMS and ${ }^{1} \mathrm{H}$ NMR analysis.

Method 4: To a solution of $\mathrm{Ph}_{3} \mathrm{P}(26.0 \mathrm{mg}, 0.1 \mathrm{mmol})$ in toluene $(8.0 \mathrm{~mL})$ was added DEAD (16 $\mu \mathrm{L}, 0.1 \mathrm{mmol}$ ) at room temperature. The reaction mixture was stirred for $30 \mathrm{~min}$ before a 114: $\mathrm{R}=\mathrm{TBDPS}$ solution of carboxylic acid $112(12 \mathrm{mg}, 0.01 \mathrm{mmol})$ in toluene $(2.0 \mathrm{~mL})$ was added via syringe pump over a period of $1 \mathrm{~h}$ at room temperature and then concentrated in vacuo. Flash column chromatography (silica gel, hexanes:EtOAc 10:1) afforded macrocycle 105 (3.6 mg, $31 \%)$ and 114 (4.6 mg, 34\%) as colorless oils. All physical properties of this compound were identical to those reported for macrocycle $\mathbf{1 0 5}$ obtained from phosphonate aldehyde 104. 


\section{References:}

(1) Myers, A.; Lanman, B. A. J. Am. Chem. Soc. 2002, 124, 12969-12971.

(2) Natural palmerolide A: $[\alpha]_{\mathrm{D}}^{25}=-99(\mathrm{MeOH}, c=0.24)$. This value is significantly higher than that reported in the original isolation paper: $[\alpha]_{\mathrm{D}}{ }^{24}=-1.6(\mathrm{MeOH}, c=0.5)$; see Diyabalanage, T.; Amsler, C. D.; McClintock, J. B.; Baker, B. J. J. Am. Chem. Soc. 2006, 128, 5630-5631. We thank Professor Bill J Baker (University of South Florida, Tampa) for kindly providing a sample of authentic palmerolide A.

(3) (a) We thank Professor Bill J Baker (University of South Florida, Tampa) for kindly providing a sample of authentic palmerolide A for NMR analysis; (b) Values obtained from the original isolation paper, see (2). 


\section{II) Abbreviations:}

$\mathrm{MOM}=$ methoxymethyl

TBS $=$ tert-butyldimethylsilyl

$\mathrm{Bn}=$ benzyl

$\mathrm{OTf}=$ trifluoromethanesulfonate

DMP $=$ Dess-Martin periodinane

DIBAL-H = diisobutylaluminum hydride

$\mathrm{TBAF}=$ tetra- $n$-butylammonium fluoride

4-DMAP = 4-dimethylaminopyridine

Piv $=$ trimethylacetyl

NaHMDS $=$ sodium hexamethyldisilazide

Ipc $=$ isopinocampheyl

NBS $=N$-bromosuccinimide

$\mathrm{dba}=$ dibenzylideneacetone,

$m$-CPBA $=m e t a-$ chloroperoxybenzoic acid

$p$-TsCl $=$ para-toluenesulfonyl chloride

$\mathrm{NMP}=N$-methylpyrrolidone

$\mathrm{DMF}=N, N^{\prime}$-dimethylformamide

TMS = trimethylsilyl

$\mathrm{PMB}=$ para-methoxybenzyl

TBDPS = tert-butyldiphenylsilyl

$p$-TsOH $=$ para-toluenesulfonic acid

$\mathrm{DCC}=N, N^{\prime}$-dicyclohexylcarbodiimide 